\title{
1979 ENVIRONMENTAL MONITORING REPORT
}

\author{
J.R. NAIDU, Editor
}

April 1980

SAFETY AND ENVIRONMENTAL PROTECTION DIVISION

BROOKHAVEN NATIONAL LABORATORY

ASSOCIATED UNIVERSITIES, INC.

UNDER CONTRACT NO. DE-AC02-76CHO0016 WITH THE

UNITED STATES DEPARTMENT OF ENERGY

$e^{2} 2 \pi$ 


\section{DISCLAIMER}

This report was prepared as an account of work sponsored by an agency of the United States Government. Neither the United States Government nor any agency Thereof, nor any of their employees, makes any warranty, express or implied, or assumes any legal liability or responsibility for the accuracy, completeness, or usefulness of any information, apparatus, product, or process disclosed, or represents that its use would not infringe privately owned rights. Reference herein to any specific commercial product, process, or service by trade name, trademark, manufacturer, or otherwise does not necessarily constitute or imply its endorsement, recommendation, or favoring by the United States Government or any agency thereof. The views and opinions of authors expressed herein do not necessarily state or reflect those of the United States Government or any agency thereof. 


\section{DISCLAIMER}

Portions of this document may be illegible in electronic image products. Images are produced from the best available original document. 
BNL 51252

UC-11

(Environmental Control Technology and Earth Sciences - TIC-4500)

\title{
1979 ENVIRONMENTAL MONITORING REPORT
}

\author{
J.R. NAIDU, Editor
}

Reviewed by: A.P. Hull

Data Compilation and Quality Control by: A.R. Moorthy and J.R. Steimers Computer Data Analysis and Tabulation by: N.J. Fallon and A.V. Kuehner
Contributors
E. Hartmann
L.L. Olmer
D.M. Henze
D.L. Smith
D.P. Higby
J.R. Steimers
L.E. McHerron
A.M. Wallner
A.R. Moorthy
V.J. White
J.A. Nobile
P.E. Wildenborg

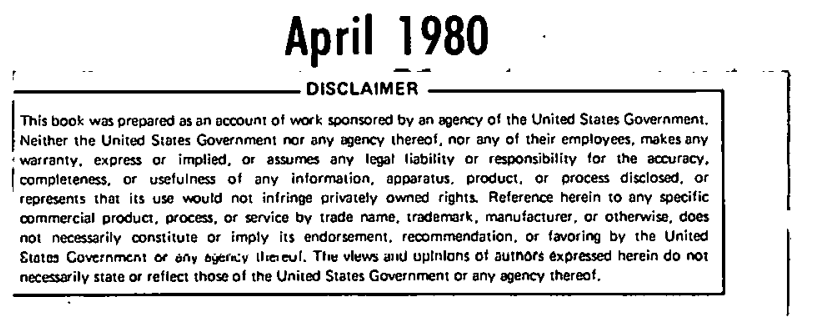

SAFETY AND ENVIRONMENTAL PROTECTION DIVISION

BROOKHAVEN NATIONAL LABORATORY UPTON, NEW YORK 11973 


\section{DISCI.AIMER}

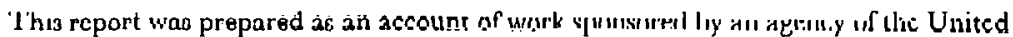
States Government. Neither the United States Government nur any agency thercof, nor any of their empluyees, wur any of their rontractors, subcontractora, or their employees, makes any warranty, express or implied, or assumes any legal liability or responsibility for the accuracy, completeness, or usefulness of any information, appara-

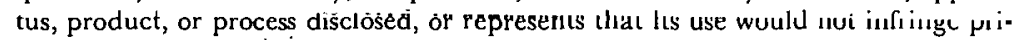
vately owned rights Referenre herein tn any specific commercial product, process, or service by trade name, trademark, manufacturer, or otherwise, does not necessarily constitute or imply its endorsement, recommendation, or favoring by the United States Government or any agency, contractor or subcontractor thereof. The views and opinions of authors expressed herein do not necessarily state or reflect those of the United States Government or any agency, contractor or subcontractor thereof.

Printed in the United States of America

$$
\text { Available from }
$$

National Technical Information Service

$$
\text { U.S. Department of Commerce }
$$

5285 Port Royal Road

Springfield, VA 22161

Price: Printed Copy $\$ 8.00$; Microfiche $\$ 3.50$ 
BROOKHAVEN NATIONAL LABORATORY

ENVIRONMENTAL MONITORING REPORT

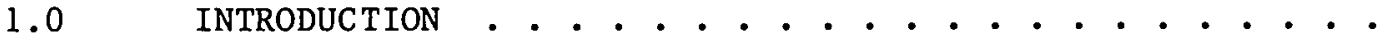

3.2.2 Sampling and Analysis .................

3.3.1 National Pollutant Discharge Elimination System

3.3.2 Meadow-Marsh Project . . . . . . . . . . . . . 28

3.3.3 Peconic River..................... 28

3.3.4 Recharge Basin .................... 39

3.3.5 Aquatic Biological Studies ............ . 43

3.3.6 Surveillance Wells ................ 45

3.3.6.1 Potable Water and Process Supply Wells . . . . . . . 45

3.3.6.2 Ground Water Surveillance . . . . . . . . . . . . . 47

3.4 Unusual Occurrences ................ . 74

3.4.1 0il Spills ....................... . . . . . . . . 74

3.4.2 Chinese Nuclear Tests . . . . . . . . . . . 76

4.0 OFF-SITE DOSE ESTIMATES . . . . . . . . . . . . 76

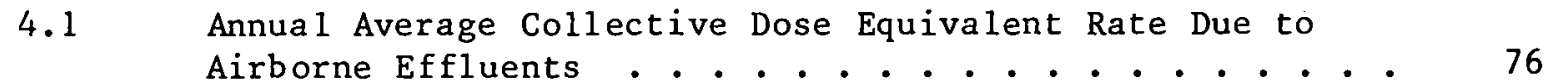

4.2 Doses Due to Liquid Effluents

4.3 Doses Due to the Gama Forest $13 i_{\mathrm{Cs}}$ Source . . . . . . . 79

4.4 Doses Due to Alternating Gradient Synchrotron . . . . . . 81

Collective Average Dose Equivalent Rate (Total
Population Dose) . . . . . . . . . . . . . 81

APPENDIX A - Quality Control ................. . 84

APPENDIX B - Minimum Detection Limit (MDL) . . . . . . . . 85 
CONTENTS (Cont'd)

$\underline{\text { Page }}$

ACKNOWLEDGMENTS . . . . . . . . . . . . . . . . . . 87

REFERENCES . . . . . . . . . . . . . . . . . . . 88

DISTRIBUTION LIST . . . . . . . . . . . . . . . . 90 
1. Map of the general Long Island area showing the location of Brookhaven National Laboratory (BNL) . . . . . . . . . 2

2. Brookhaven National Laboratory Site . . . . . . . . . 4

3. Annual Wind Rose .................. . . 5

4. Schematic Ground-Water Flow Lines, Central Upton Area . . . 7

5. BNL Liquid Effluent Systems . . . . . . . . . . . . . 27

6. Sewage Treatment Plant ............... . . . 30

7. Peconic River, On-Site and Downstream Sampling Locations . • 37

8. On-Site Potable and Supply Wells . . . . . . . . . . 49

9. Location of Ground Water Surveillance Wells . . . . . . . 50

10. Landfill and Waste Management Area Surveillance Wells . . . 51

11. Direction and Time of Travel of Ground Water Laterally in Upper Pleistocene Deposits from BNL Area to Points of Discharge . . . . . . . . . . . . . . . . 75 
1. 1979 BNL Environmental Monitoring: Resident Population (1979) Distribution within $80 \mathrm{~km}$ Radius of BNL . . . . . . . . . 3

2. 1979 BNL Environmental Monitoring: Weekly External Dose Equivalent Rates from Background and BNL Operations . . . . . . 13

3. 1979 BNL Environmental Monitoring: Gaseous Effluent Release Locations and On-Line Monitoring and Sampling Devices . . . . 15

4. 1979 BNL Environmental Monitoring: Airborne Effluent Data Radioactive Effluents . . . . . . . . . . . . . . . . 16

5. 1979 BNL Environmental Monitoring: Emission of $\mathrm{SO}_{2}, \mathrm{NO}_{\mathrm{x}}$ and Particulates from Central Steam Plant (B1dg. 610) . . . . . 18

6. 1979 BNL Environmental Monitoring: Average Gross Alpha and Gross Beta Concentrations, Air Particulate Filters . . . . . 21

7. 1979 BNL Environmental Monitoring: Monthly Average Concentrations of Gross Beta Activity and of Gamma Emitting Nuclides in Monthly Composite Air Particulate and Charcoal Filters . . . . . . . . . . . . . . . . . . . . .

8. 1979 BNL Environmental Monitoring: Average Tritium Vapor Concentrations in Air . . . . . . . . . . . . . . . . . 24

9. 1979 BNL Environmental Moritoring: Monthly Average Gross Beta Concentration, Total Gross Beta Activity and Individual Nuclide Activity in Precipitation . . . . . . . . . . .

10. 1979 BNL Environmental Monitoring: National Pollution Discharge Elimination System - Sumary of Monthly Reports . . .

11. 1979 BNL Environmental Monitoring: Total Activities and Concentrations of Identifiable Nuclides in Liquid Effluents - Sewage Treatment Plant . . . . . . . . . .

12. 1979 BNL Environmental Monitoring: Total Activities and Concentrations of Identifiable Nuclides in Liquid Effluents - Peconic River .................

13. 1979 BNL Environmental Monitoring: Liquid Effluent (River, Sewage) and Precipitation - Water Quality and Metals . . . . 35

14. 1979 BNL Environmental Monitoring: Downstream and Control Water Samples - Gross Beta, Tritium (HTO) and ${ }^{90} \mathrm{Sr}$. . . . . 
15. 1979 BNL Environmental Monitoring: Downstream and Control Water Samples - Water Quality and Metals . . ... . . . 40-41

16. 1979 BNL Environmental Monitoring: Monthly Sump Samples Gross Beta and $3_{\mathrm{H}}$ (HTO) Concentrations . . . . . . . . . 42

17. 1979 BNL Environmental Monitoring: Recharge Basins - Water Quality and Metals . . . . . . . . . . . . . . . .

18. 1979 BNL Environmental Monitoring: Concentration of $90_{\mathrm{Sr}}$ and

$137 \mathrm{Cs}$ in Water, Sediment, Vegetation and Fish obtained from the Peconic River at the Site Perimeter as observed during 1974-1979

19. 1979 BNL Environmental Monitoring: Gross Beta and Tritium Concentrations in Potable Water and Cooling Water Supply Wells . . . . . . . . . . . . . . . . . . . .

20. 1979 Environmental Monitoring: Sand Filter Bed, Peconic River Area, and Miscellaneous On-Site Surveillance Wells Gross Alpha, Gross Beta, Tritium (HTO), $90 \mathrm{Sr}$ and other Nuclides-Average Concentrations

21. 1979 BNL Environmental Monitoring: Waste Management, Landfi11, Former Dump and 650 Area Surveillance Wells.Gross Alpha, Gross Beta, Tritium (HTO), ${ }^{90} \mathrm{Sr}$ and other Radionuclides - Average Concentrations $55-58$

22. 1979 BNL Environmental Monitoring: Sand Filter Beds, Peconic River and Miscellaneous On-Site Wells - Water Quality and Purity .. . . . . . . . . . . . . . . 61-62

23. 1979 BNL Environmental Monitoring: Solid Waste Management, Landfil1, Former Dump and 650 Sump Area Wells - Water Quality and Purity . . . . . . . . . . ... . . . 63-65

24. 1979 BNL Environmental Monitoring: Cooling Water Weils, Tap Water and Potable Supply Wells - Water Quality and Purity . .

25. 1979 BNL Environmental Monitoring: Sand Filter Beds, Peconic Kiver, Miscellaneous On-Site, Waste Management, 650 Sump, Landfill, and Former Dump Area Wells - Metals . . . . . 67-72

26. 1979 BNL Environmental Monitoring: Potable and Cooling Water Supply Wells - Metals ................... 
27. 1979 BNL Environmental Monitoring: Collective Annual Average Dose Equivalent Rate Due to BNL Airborne Effluents in Comparison with Background . . . . . . . . . . . . . .

28. 1979 BNL Environmental Monitoring: Off-Site Collective Annual Average Dose Equivalent Rate Due to External Radiation Exposure resulting from the Gamma Forest and AGS Operations . . . . . . . . . . . . . . . . . . .

29. Maximum Permissible Levels of Contaminants in Air and Water with their Detection Limits . . . . . . . . . . . . . . . 


\subsection{INTRODUCTION}

\section{.1 Background:}

The primary purpose of a routine environmental monitoring program, according to DOE Manual Chapter 0513(1), is to determine whether:

1) facility operations, waste treatment, and control systems have functioned as designed and planned from the standpoint of containment of radioactivity, and

2) the applicable environmental radiation and radioactivity standards and effluent control requirements have been met.

Brookhaven National Laboratory's (BNL) environmental monitoring program is designed and developed to accomplish these two primary objectives. While this annual report follows the recommendations given in ERDA 77-24, "A Guide for Environmental Radiological Surveillance at DOE's Installations" (2), considerable latitude has been exercised in tailoring the scope and methodology to meet the site's specific environmental monitoring needs. In addition, the Laboratory has extended its surveillance program to include analysis of the environment for non radiological components such as heavy metals and organics. This latter program is being regularly updated to reflect the growing concern about nonradiological pollutants.

\subsection{Site Characteristics:}

Brookhaven National Laboratory is a multidisciplinary scientific research center situated in the geographical center of Suffolk County on Long Island, about $97 \mathrm{~km}$ east of New York City. Its location with regard to surrounding communities is shown in Figure 1. About $1.3 \mathrm{million}$ people live in Suffolk

County $(3,4)$. The principal nearby population centers are located in shoreline communities. Table 1 gives the resident population distribution within $80 \mathrm{~km}$ of the BNL site. Though much of the land area with in a $16 \mathrm{~km}$ radius is either forested or under cultivation, there is a transition towards development of suburban housing in proximity to the Laboratory.

The Laboratory site is shown in Figure 2. It consists of some 2130 ha, most of which is wooded, except for a developed area of about 655 ha. The site terrain is gently rolling, with elevations varying between 36.6 and $13.3 \mathrm{~m}$ above sea level. The land lies on the western rim of the shallow Peconic River watershed, with the river itself rising in marshy areas in the north and east sections of the site.

In terms of meteorology, the Laboratory can be characterized as a wellventilated site. In common with most of the eastern seaboard, its prevailing ground level winds are from the southwest during the summer of the year, from the northwest during the winter, and about equally from these two directions during the spring and fall. This is reflected in the annual wind distribution at an elevation of $108 \mathrm{~m}$, as observed by the BNL Meteorology Group, which is shown in Figure 3 . 


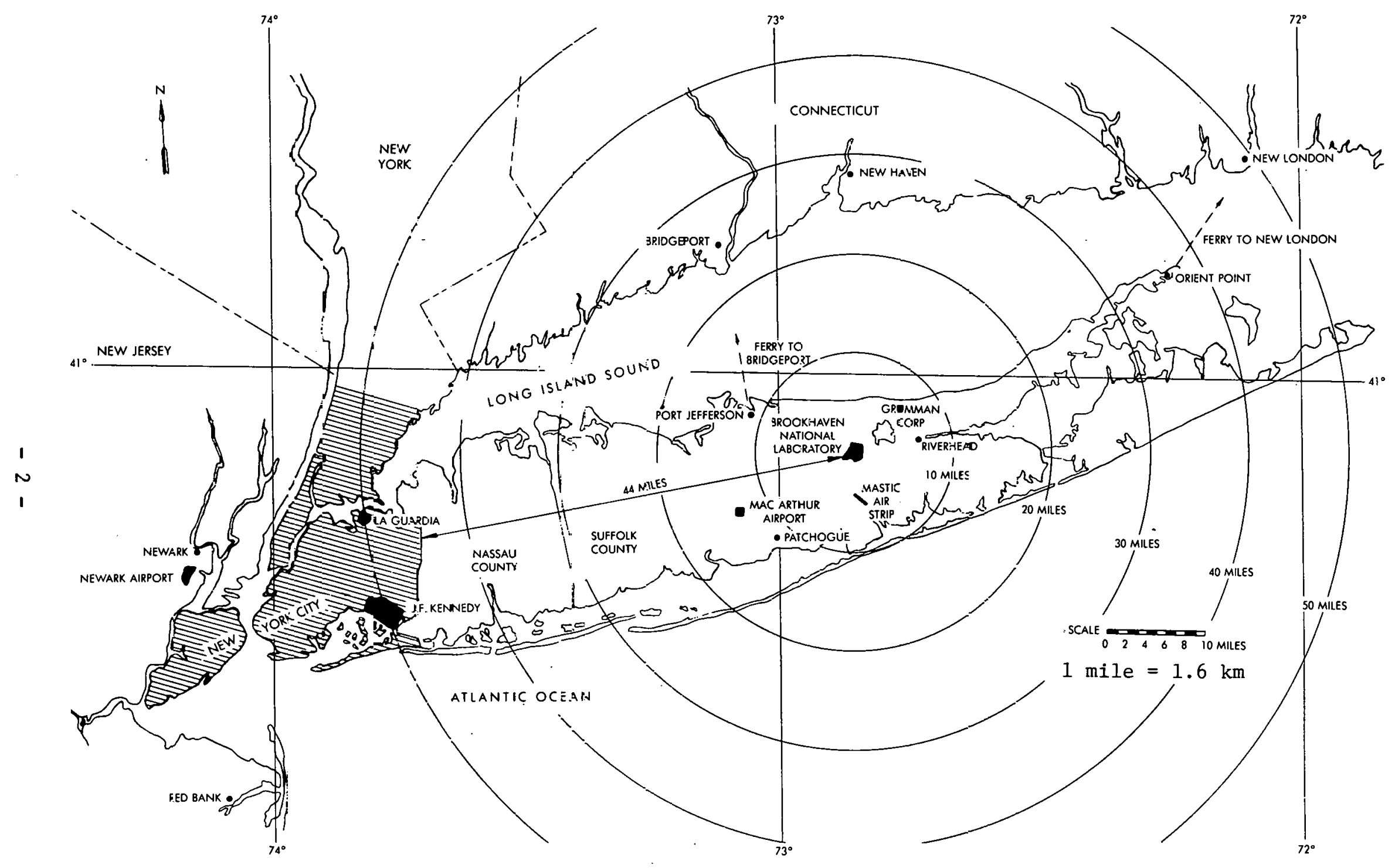

Figure 1. Map of the General Long Island Area Shoving the Locażon of Brookhaven National Labcratory 
TABLE 1

1979 BNL Environmental Monitoring Resident Population (1979) a Distribution Within $80 \mathrm{~km}$ Radius of BNL

\begin{tabular}{|c|c|c|c|c|c|c|c|}
\hline Sector & $\begin{array}{l}0- \\
16 \mathrm{~km} \\
(10 \mathrm{mi})\end{array}$ & $\begin{array}{l}16- \\
32 \mathrm{~km} \\
(20 \mathrm{mi})\end{array}$ & $\begin{array}{l}32- \\
43 \mathrm{~km} \\
(30 \mathrm{mi})\end{array}$ & $\begin{array}{l}48- \\
64 \mathrm{~km} \\
(40 \mathrm{mi})\end{array}$ & $\begin{array}{l}64- \\
80 \mathrm{~km} \\
(50 \mathrm{mi})\end{array}$ & Total & Remarks \\
\hline SSW & 19,496 & 998 & 0 & 0 & 0 & 20,494 & Beyond $32 \mathrm{~km}$ - Atlantic Ocean \\
\hline SW & 38,388 & 59,698 & 3,165 & 0 & 0 & 101,251 & Beyond $48 \mathrm{~km}$ - Atlantic Ocean \\
\hline WSW & 34,619 & 132,802 & 327,784 & 426,904 & 764,169 & $1,686,278$ & Beyond $80 \mathrm{~km}$ - Part of New York City \\
\hline W & 45,216 & 122,556 & 220,678 & 227,073 & 369,930 & 985,453 & Beyond $80 \mathrm{~km}$ - New York City \\
\hline WNW & 38,103 & 53,083 & 100 & 201,804 & 121,881 & 414,971 & $\begin{array}{l}\text { Between } 32 \mathrm{~km} \& 48 \mathrm{~km} \text { - Long Island } \\
\text { Sound and beyond } 48 \mathrm{~km} \text { - Connecticut and } \\
\text { New York States. }\end{array}$ \\
\hline NW & 16,413 & 1,425 & 127,910 & 115,460 & 105,044 & 366,252 & Same as WNW \\
\hline NNW & 6,995 & 0 & 197,286 & 101,199 & 51,197 & 356,677 & $\begin{array}{l}\text { Between } 16 \mathrm{~km} \text { and } 32 \mathrm{~km} \text { - Long Island } \\
\text { Sound, beyond } 20 \mathrm{~km} \text { - Connecticut. }\end{array}$ \\
\hline $\mathrm{N}$ & 4,143 & 0 & 38,352 & 234,167 & 244,355 & 571,017 & Same as NNW \\
\hline NNE & 6,905 & 0 & 6,526 & 41,783 & 61,738 & 116,952 & Same as NNW \\
\hline $\mathrm{NE}$ & 2,685 & 682 & 0 & 12,625 & 30,869 & 46,861 & $\begin{array}{l}\text { Between } 32 \mathrm{~km} \text { and } 48 \mathrm{~km} \text { - Long Island } \\
\text { Sound, beyond - Connecticut }\end{array}$ \\
\hline ENE & 2,275 & 6,408 & 11,849 & 13,450 & 2,022 & 36,004 & North Fork of Long Island \\
\hline E & 2,755 & 14,424 & 15,702 & 8,192 & 501 & 41,574 & $\begin{array}{l}\text { South Fork on Long Island and Atlantic } \\
\text { Ocean. }\end{array}$ \\
\hline ESE & 5,608 & 6,945 & 0 & 0 & 0 & 12,553 & $\begin{array}{l}\text { Long Island and Beyond } 32 \mathrm{~km} \text { - Atlantic } \\
\text { Ocean. }\end{array}$ \\
\hline SE & 8,270 & 0 & 0 & 0 & 0 & 8,270 & Beyond $18 \mathrm{~km}$ - Atlantic Ocean \\
\hline SSE & 20,414 & 0 & 0 & 0 & 0 & 20,414 & Same as SE \\
\hline S & 15,107 & 18 & 0 & 0 & 0 & 15,125 & Beyond $32 \mathrm{~km}$ - Atlantic Ocean \\
\hline Total & 257,392 & 399,039 & 999,352 & $1,382,657$ & $1,751,706$ & $4,800,146$ & \\
\hline
\end{tabular}

apopulation data estimated from information supplied by Ms. Peggy Wagner, Research Analyst, Long Island Regional Planning Board $[3,4]$. 


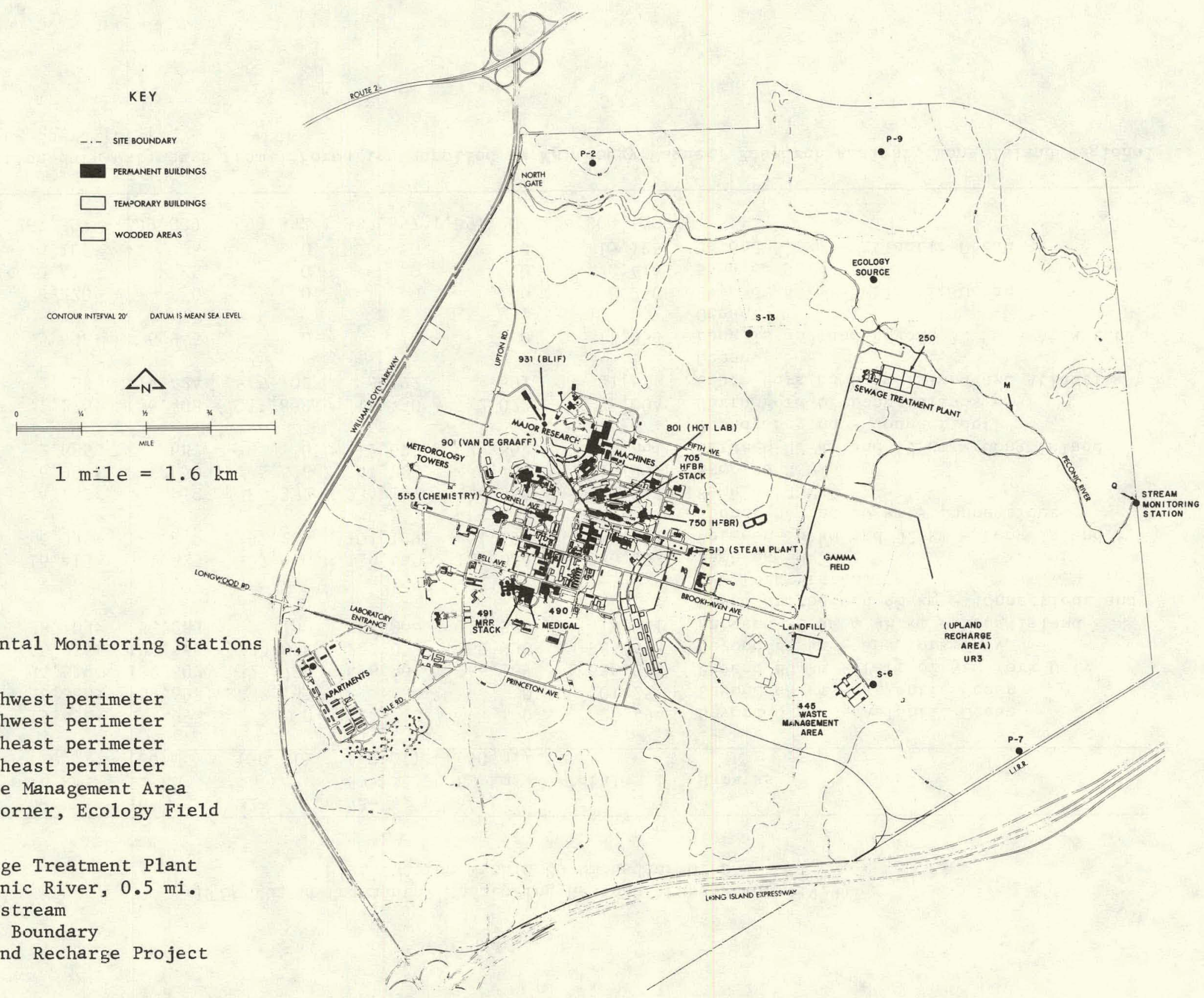

Figure 2. Brookhaven National Laboratory Site 


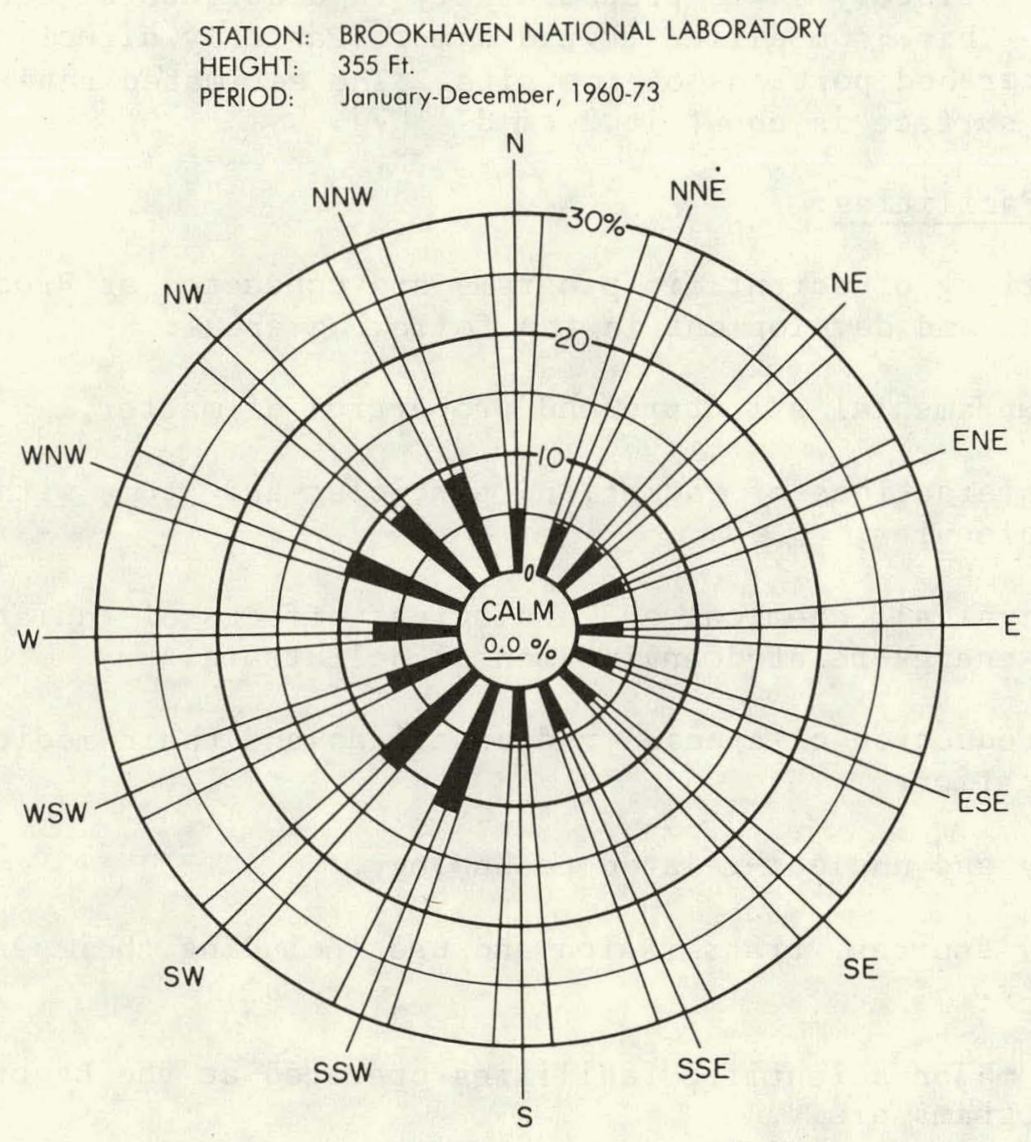

Figure 3. Annual Wind Ruse. The Wind Ruse also Represents the Period 1973-1979 
Studies of the hydrology and geology (5-7) of Long Island in the vicinity of the Laboratory indicate that the uppermost Pleistocene deposits, which are 1o cally between 31-61 m thick, are generally sandy and highly permeable. Water penetrates them readily and there is little direct run-off into surface streams except during periods of intense precipitation. The average annual precipitation is $122 \mathrm{~cm}$ and the annual total for 1979 was $142.5 \mathrm{~cm}$. About half of it is lost to the atmosphere through evapotranspiration and the other half percolates to recharge ground water. As indicated in Figure 4 (7), the ground water in the vicinity of the Laboratory moves predominantly in a horizontal direction to the Great South Bay. This is modified toward a more easterly direction in the Peconic River watershed portions of the site. The estimated rate of movement at the ground water surface is about $16.2 \mathrm{~cm} \mathrm{~d}^{-1}(7)$.

\subsection{Existing Facilities:}

A wide variety of scientific programs are conducted at Brookhaven, including research and development in the following areas:

1) the fundamental structure and properties of matter,

2) the interactions of radiation, particles and atoms with other atoms and molecules,

3) the physical, chemical and biological effects of radiation, and of other energy-related environmental pollutants,

4) the production of special radionuclides and their medical applications,

5) energy and nuclear-related technology,

6) energy sources, transmission and use including their environmental effects.

Among the major scientific facilities operated at the Laboratory to carry out the above programs are:

1) the High F1ux Beam Reactor (HFBR) which is fueled with enriched uranium, moderated and cooled by heavy water, and which operates at a routine power level of $40 \mathrm{MW}(\mathrm{th})$. Recently, modifications to the primary water cooling system have been made to allow the power level to be raised to $60 \mathrm{MW}(\mathrm{th})$ in the immediate future,

2) the Medical Research Reactor (MRR), an integral part of the Medical Research Center (MRC), is fueled with enriched uranium, moderated and cooled by natural water, and is operated intermittently at power levels up to $3 \mathrm{MW}(\mathrm{th})$,

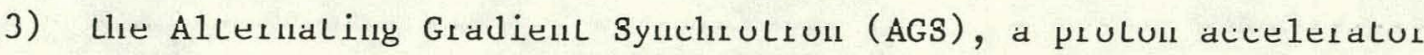
which operates at energies up to $33 \mathrm{GeV}$, 


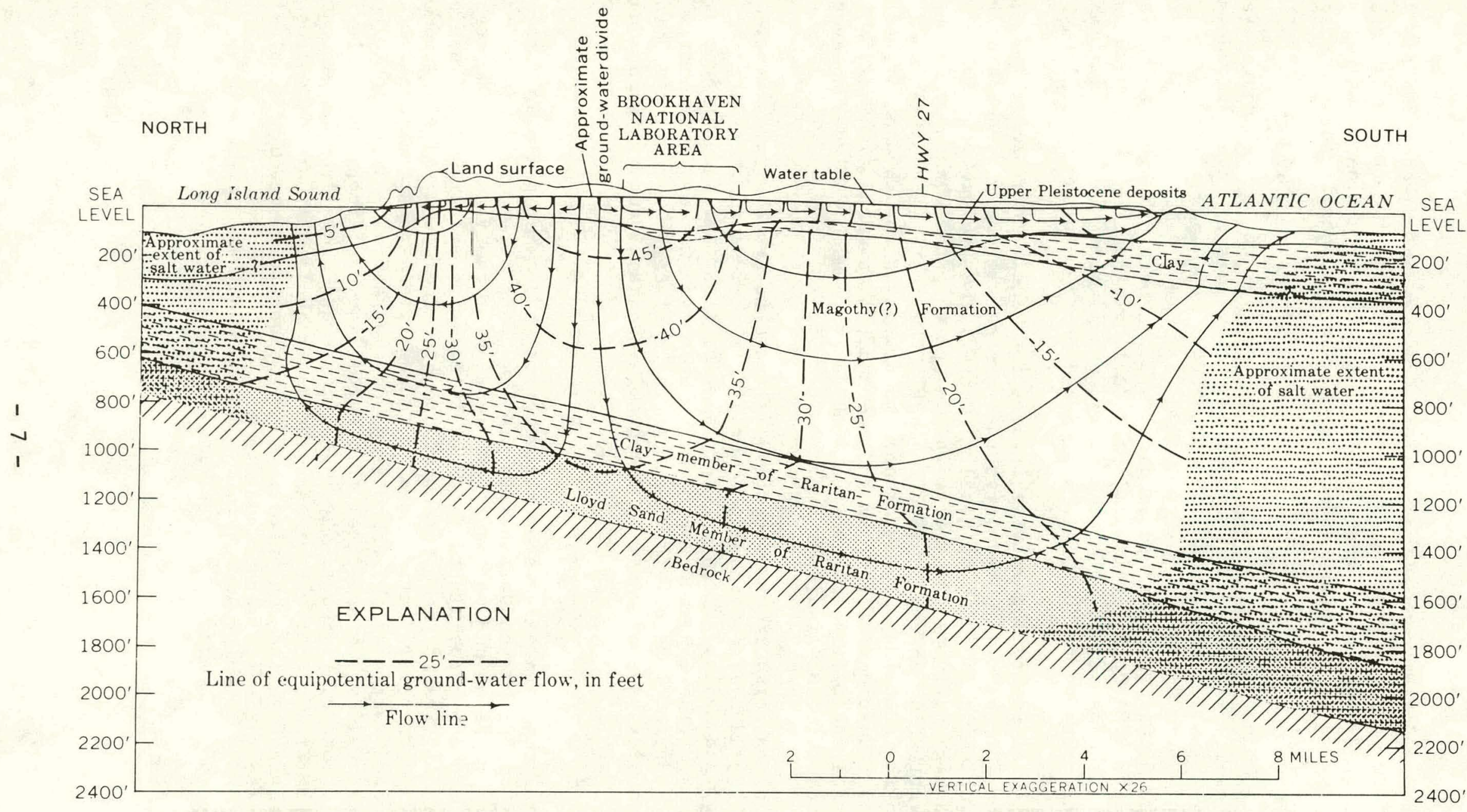

Figure 4. Schematic Ground-Water Flow Lines, Central Upton Area 
4) the $200 \mathrm{MeV}$ Proton Linac, which serves as an injector for the AGS, also supplies continuous currents of protons for radionuclide production by spallation reactions in the Brookhaven Linac Isotopes Production Facility (BLIP) and in the Chemistry Linac Irradiation Facility (CLIF),

5) the Tandem Van de Graaff, Vertical Accelerator and Chemistry Van de Graaff, which are used in medium energy physics investigations, as well as for special nuclide production.

6) To facilitate advanced studies in high energy physics, a new facility, "ISABELLE," which will be a colliding-beam machine with two proton beams of $400 \mathrm{GeV}$ each and whose collision will make available energies up to $800 \mathrm{GeV}$, is propnsed for completion hy 1983 (8).

Additional programs involving irradiations and/or the use of radionuclides for scientific investigations are carried on at other Laboratory facilities including the Medical Research Center, the Biology Department (including a high activity gamma irradiation source), the Chemistry Department, and the Department of Energy and Environment (DEE). At the Hot Laboratory special purpose radionuclides are developed and processed for on- and off-site use under the joint auspices of the DEE and the Medical Department. This facility also contains a radioactive waste treatment center, which includes an evaporator for volume reduction of liquid wastes.

Most of the airborne radioactive effluents at Brookhaven originate from the HFBR, BLIP and the research Van de Graaff, with lesser contributions from the Chemistry and Medical Research Centers. The first two also produce significant fractions of the Laboratory's liquid radioactive wastes, with additional significant contributions originating from the Medical Research Center, the Hot Laboratory complex, as we 11 as from decontamination and laundry operations.

\subsection{SUMMARY}

The environmental levels of radioactivity and other pollutants found in the vicinity of Brookhaven National Laboratory (BNL) during 1979 are summarized in this report. As an aid in the interpretation of the data, the amounts of radioactivity and other pollutants released in airborne and liquid effluents from Laboratory facilities to the environment are also indicated. The environmental data includes external radiation levels; radioactive air particulates; tritium and iodine concentrations; the amounts and concentrations of radioactivity in and the water quality of the stream into which liquid effluents are released; the concentrations of radioactivity in sediments and biota from the stream; the concentrations of radioactivity in and the water quality of ground waters under1ying the Laboratory; and concentrations of radioactivity in milk samples obtained in the vicinity of the Laboratory. 
The external radiation dose for 1979 at the north boundary of the Laboratory attributable to an ecology forest irradiation source was $1.43 \mathrm{mRem} \mathrm{a}^{-1}$ $\left(1.43 \times 10^{-5} \mathrm{~Sv} \mathrm{a}^{-1}\right)$ or $0.4 \%$ of the applicable Radiation Protection Standard.*

At the boundary of the Laboratory, about $1.0 \mathrm{~km}$ northwest of the Alternating Gradient Synchrotron (AGS), the calculated dose due to skyshine (scattered radiation) was about $0.88 \mathrm{mRem}^{-1}\left(0.88 \times 10^{-5} \mathrm{~Sv} \mathrm{a}^{-1}\right)$, or $0.16 \%$ of the Standard. This was too small to be measured. Due to their limited range, the external radiation from the AGS and those from the gamma forest source did not produce a measurable additive effect at off-site locations.

Other than tritium, there was no indication of BNL radioactive effluents in environmental air and precipitation samples. The largest concentration of tritium in air at the site boundary, $7 \mathrm{pCi} \mathrm{m}^{-3}\left(0.7 \times 10^{-2} \mu \mathrm{Ci} \mathrm{ml}^{-1}\right.$ or $0.26 \times$ $10^{-5} \mathrm{~Bq} \mathrm{~m} 1^{-1}$ ) was $<0.01 \%$ of the Radiation Concentration Guide (RCG). The largest average concentration of tritium in precipitation was at or below the Minimum Detection Limit (MDL) which was $160 \mathrm{pCi} 1^{-1}\left(1.6 \times 10^{-7} \mu \mathrm{Ci} \mathrm{ml}^{-1}\right.$ or 5.9 $\left.x 10^{-3} \mathrm{~Bq} \mathrm{ml}-1\right)$. The MDL represents about $0.01 \%$ of the RCG for drinking water.

At the Central Steam Plant, the most recent measurement of the stack emission of air particulates indicated that the average rate was $0.0781 \mathrm{~b} / 10^{6} \mathrm{Btu}$. A calculation based on meteorological parameters indicates that at the site boundary, their concentration was $0.35 \mu \mathrm{g} \mathrm{m}^{-3}, 0.48 \%$ of the yearly average ambient Air Quality Standard (12). At the site boundary the calculated concentrations of $\mathrm{SO}_{2}, \mathrm{NO}_{\mathrm{x}}$, Lead $(\mathrm{Pb})$ and Cadmium $(\mathrm{Cd})$ emitted from the plant were $1.1 \mathrm{x}$ $10^{-3}, 6 \times 10^{-4}, 5 \times 10^{-9}$ and $7.6 \times 10^{-12} \mathrm{ppm}$, respectively, which were $4.7,1.6$, 0.3 and $<0.01 \%$ of their respective ambient air quality standards.

of the sewage effluent released onto the sand filter beds of the Laboratory sewage treatment plant $78 \%$ flowed directly into the Peconic River. The balance was assumed to have percolated into the ground water underlying the beds. The gross beta concentration of the output from them was $28.5 \mathrm{pCi} 1-1(2.85 \mathrm{x}$ $10^{-8} \mu \mathrm{Ci} \mathrm{m1}-1$ or $\left.1.06 \times 10^{-3} \mathrm{~Bq} \mathrm{ml}-1\right)$, or $<1 \%$ of the RCG. The tritium concentration was $11.9 \mathrm{nCi} 1^{-1}\left(11.9 \times 10^{-6} \mu \mathrm{Ci} \mathrm{ml}-1\right.$ or $\left.4.4 \times 10^{-1} \mathrm{~Bq} \mathrm{ml}-1\right)$, or $0.6 \%$ of the RCG. The same concentration was assumed for the infiltration into groundwater.

Downstream about $3.5 \%$ of the combined flow from the sand filter beds and from upstream of the Peconic River also percolated into the groundwater. This occurred between the sewage treatment plant outfall and the Laboratory perimeter, mostly during the latter half of the year. As established at a midway stream sampling location, the gross beta concentration was $11.7 \mathrm{pCi} 1^{-1}(1.17 \mathrm{x}$ $10^{-8} \mu \mathrm{Ci} \mathrm{ml}^{-1}$ or $\left.4.3 \times 10^{-4} \mathrm{~Bq} \mathrm{ml}^{-1}\right)$, or $<1 \%$ of the RCG, and the tritium concentration was $4.9 \mathrm{nCi} 1^{-1}\left(4.9 \times 10^{-6} \mu \mathrm{Ci} \mathrm{m1}-1\right)$, or $0.1 \%$ of the RCG. At the site boundary, the gross beta concentration was $15.4 \mathrm{pCi} 1^{-1}\left(1.54 \times 10^{-8} \mu \mathrm{Ci} \mathrm{ml}^{-1}\right.$ or

*The applicable Radiation Protection Standards and Radiation Concentration Guides for persons in uncontrolled areas are shown with the relevant tabulated data. 
$\left.0.53 \times 10^{-3} \mathrm{~Bq} \mathrm{m1}-1\right)$, or $0.5 \%$ of the RCG, and the tritium concentration was 5.4 $\mathrm{nCi} 1^{-1}\left(5.4 \times 10^{-6} \mu \mathrm{Ci} \mathrm{m1}-1\right.$ or $\left.2 \times 10^{-1} \mathrm{~Bq} \mathrm{~m} 1^{-1}\right)$, or $0.17 \%$ of the RCG.

Except for 35 daily $\mathrm{pH}$ levels which were "out of limit," all reportable non-radiological parameters of the Laboratory sewage effluent were within the limits set forth in the Laboratory's permit, issued by EPA under the National Pollution Discharge Elimination System. The average water quality of the sewage treatment plant effluent at the point of discharge was at or within water quality standards for the receiving body of water.

Bimonthly sampling of the Peconic River water downstream of the sewage treatment plant outfall has indicated a decrease of concentrations of radioactivity. At a location $4.8 \mathrm{~km}$ downstream, the average gross beta concentration as established by bimonthly "grab" sampling was $8 \mathrm{pCi} 1^{-1}\left(8 \times 10^{-9} \mu \mathrm{Ci}\right.$ $\mathrm{ml}^{\cdots 1}$ or $3 \times 10^{\cdots 4} \mathrm{~Bq} \mathrm{mi} \mathrm{l}^{\cdots 1}$ ), or $0.20 \%$ of the $\mathrm{KCG}$ and the tritium concentration lese than $0.57 \mathrm{pCi}^{-1}\left(<0.57 \times 10^{-6} \mu \mathrm{Ci} \mathrm{ml}-1\right.$ or $\left.<2.1 \times 10^{-2} \mathrm{~Bq} \mathrm{ml}{ }^{-1}\right)$, or $0.01 \%$ of the RCG. About $24 \mathrm{~km}$ downstream, at the river's mouth, the flow was about 19 times that at the Laboratory's site boundary (USGS-1979 data), the average concentration of gross beta activity being $6.7 \mathrm{pCi} 1^{-1}\left(6.7 \times 10^{-9} \mu \mathrm{Ci} \mathrm{m1} 1^{-1}\right.$ or 2.5 $\left.\mathrm{x} 10^{-1} \mathrm{~Bq} \mathrm{ml}-1\right)$ and that of tritium being $0.8 \mathrm{nCi} 1^{-1}\left(8 \times 10^{7} \mu \mathrm{Ci} \mathrm{ml}^{-1}\right.$ or 2.8 $\left.x 10^{-2} \mathrm{~Bq} \mathrm{~m} 1^{-1}\right)$. Thus, it was apparent that the total gross beta activity in the river at that location exceeded that at the Laboratory's site boundary. This difference is attributed to the fact that the total flow at the river's mouth is increased due to tributary additions which in turn have added fallout radionuclides that were present in the drainage area of the tributaries.

Seasonal sampling of Peconic River bottom sediments, stream vegetation and of miscellaneous aquatic fauna was conducted. The data indicated that concentration of ${ }^{51} \mathrm{Cr},{ }^{60} \mathrm{Co}$ and $65 \mathrm{Zn}$, which are unique to the Laboratory's effluents, as we 11 as ${ }^{22} \mathrm{Na},{ }^{137} \mathrm{Cs}$ and ${ }^{144} \mathrm{Ce}$, which may also represent fallout contributions, were below the Minimum Detection Limits (MDL) of the system used and as such were not reported. The data on fish obtained from the river at the site boundary suggested the presence of small amounts of radioactivity attributable to the Laboratory's past releases. The concentration of $137 \mathrm{Cs}$ ranged from 1036 to $1113 \mathrm{pCi} \mathrm{kg-1}\left(4 \times 10^{1}\right.$ to $\left.37 \mathrm{~Bq} \mathrm{~kg}-1\right)$. This concentration was 0.03 to $0.02 \%$ of the RCG, based on an assumed ingestion of $50 \mathrm{~g} \mathrm{~d}^{-1}$.

About 19 million liters of water per day obtained from on-site supply wells were used for "once through" cooling and returned to groundwater in onsite recharge basins. The concentration of gross beta activity in them was about five to ten times greater than that of the supply wells, and was less than $0.1 \%$ of the RCG. The tritium concentrations were less than the MDL, which is about $0.1 \%$ of the RGG.

Groundwater surveillance was conducted in a network of some 87 sampling wells installed adjacent to and downstream from identified areas where there is a potential for the percolation to and migration of radioactivity and other contaminants in groundwater. Immediately adjacent to the sand filter beds and to the Peconic River on-site and at the site boundary, gross beta, tritium and ${ }^{90} \mathrm{Sr}$ concentrations have been decreasing, when compared to those observed during pre- 
vious years. This reflects the decrease in the concentrations due to decay and dilution. They were not more than a few percent of the EPA Drinking Water Standards. The largest average gross alpha concentration, $6 \mathrm{pCi} 1^{-1}\left(6 \times 10^{-9} \mu \mathrm{Ci}\right.$ $\mathrm{m}^{-1}$ or $2.1 \times 10^{-4} \mathrm{~Bq} \mathrm{ml^{-1 }}$ ) was $40 \%$ of the EPA Drinking Water Stundard for unidentified mixtures containing alpha activity other than ${ }^{226} \mathrm{Ra}$. It was not directly relatable to any known Laboratory effluent releases. The largest average gross beta concentration was $20 \mathrm{pCi} 1^{-1} 2 \times 10^{-8} \mu \mathrm{Ci} \mathrm{m1^{-1 }}$ or $\left.0.7 \times 10^{-3} \mathrm{~Bq} \mathrm{ml}^{-1}\right)$. The 1 argest average tritium concentration, $3.1 \mathrm{nCi} 1^{-1}\left(3.1 \times 10^{-6} \mu \mathrm{Ci} \mathrm{ml^{-1 }}\right.$ or $1.2 \times 10^{-1} \mathrm{~Bq} \mathrm{ml}-1$ ) was $21 \%$ of the EPA Drinking Water Standard.

Concentrations of gross alpha, gross beta and $90 \mathrm{Sr}$ radioactivity were found to be slightly higher in a sampling we 11 about $0.35 \mathrm{~km}$ east of the site boundary, than in wells at the boundary itself. The gross alpha concentration, $3.3 \mathrm{pCi}^{-1}\left(3.3 \times 10^{-9} \mu \mathrm{Ci} \mathrm{m}{ }^{-1}\right.$ or $\left.12.3 \times 10^{-6} \mathrm{~Bq} \mathrm{ml}-1\right)$ was $22 \%$ of the EPA Drinking Water Standard. However, this was not directly relatable to any known Laboratory effluent. The gross beta concentration was $15.2 \mathrm{pCi} 1^{-1}\left(15.2 \times 10^{-9}\right.$ $\mu \mathrm{Ci} \mathrm{ml}^{-1}$ or $\left.5.7 \times 10^{-4} \mathrm{~Bq} \mathrm{m1}{ }^{-1}\right)$, and the ${ }^{90} \mathrm{Sr}$ concentration was $2 \mathrm{pCi} 1^{-1}(2 \mathrm{x}$ $10^{-9} \mu \mathrm{Ci} \mathrm{m} \mathrm{m}^{-1}$ or $\left.0.8 \times 10^{-4} \mathrm{~Bq} \mathrm{ml}{ }^{-1}\right)$. The latter was $25 \%$ of the EPA Drinking Water Standard.

Except for $\mathrm{pH}$ levels slightly lower than the Water Quality Standard, but within the local natural variation, most other indices of water quality in these surveillance wells were with in the standards. In a few on-site wells immediately adjacent to the sand filter beds and to the Peconic River on-site, Fe and $\mathrm{Zn}$ were found up to ten times their respective water quality standards. These levels exceeded those found in recent Laboratory liquid effluents, and may be an artifact produced by the sampling we 11 casing rather than being present in groundwater itself.

On-site, adjacent to the Solid Waste Management area, the landfill, the former open dump, and the decontamination facility storm sewer sump, above ambient background concentrations of gross beta activity, $90 \mathrm{Sr}$, and tritium were found in a number of nearby groundwater surveillance wells. Much of the gross beta activity appeared to be related to $90 \mathrm{Sr}$.

At the Waste Management area, the 1 argest $90 \mathrm{Sr}$ concentration, $107 \mathrm{pCi}$ $1^{-1}\left(10.7 \times 10^{-10} \mu \mathrm{Ci} \mathrm{m}^{-1}\right.$ or $\left.4.0 \times 10^{-3} \mathrm{~Bq} \mathrm{ml}-1\right)$, or 13 times the EPA Drinking Water Standard, was found in a we $11175 \mathrm{~m}$ south of the area. This level reflects the effects of a known inadvertent injection into groundwater which occurred in 1960.

At the landfill, a gross alpha concentration of $42.5 \mathrm{pCi} 1^{-1}(4.25 \mathrm{x}$ $10^{-8} \mu \mathrm{Ci} \mathrm{ml^{-1 }}$ or $\left.1.8 \times 10^{-6} \mathrm{~Bq} \mathrm{~m} 1^{-1}\right)$, or $300 \%$ of the EPA Drinking Water Standard (18), a gross beta concentration of $119 \mathrm{pCi} 1^{-1}\left(1.19 \times 10^{-7} \mu \mathrm{Ci} \mathrm{ml}-1\right.$ or $4.4 \mathrm{x}$ $\left.10^{-3} \mathrm{~Bq} \mathrm{ml}-1\right)$, or $6 \%$ of the RCG, and a tritium concentration of $427 \times 10^{-6} \mu \mathrm{Ci}$ $\mathrm{ml}^{-1}\left(1.5 \times 10^{1} \mathrm{~Bq} \mathrm{ml^{-1 }}\right)$, or 21 times the EPA Drinking Water Standard, were the largest found. They were found in wells between the 1 andfill and locations 80 $\mathrm{m}$ south of the perimeter of the working area.

At the decontamination facility storm sewer sump, a ${ }^{90} \mathrm{Sr}$ concentration of $57.1 \mathrm{pCi} 1^{-1}\left(5.71 \times 10^{-8} \mu \mathrm{Ci} \mathrm{m1} 1^{-1}\right.$ or $\left.20.7 \times 10^{-3} \mathrm{~Bq} \mathrm{ml}-1\right)$, or 650 times the EPA 
Drinking Water Standard, was found in a surveillance we 11 about $50 \mathrm{~m}$ southeast of the sewer outfall into the sump.

With the exception of the presence of $\mathrm{Fe}$ and $\mathrm{Zn}$ in wells adjacent to the 1 andfill area, all on-site water quality and purity parameters were within the established standards. Immediately adjacent to the landfill, the concentration of $\mathrm{Fe}$ was $49 \mathrm{ppm}$, or 86 times the standard, and that of $\mathrm{Zn}$ was $2.7 \mathrm{ppm}$, or 4.3 times the standard.

In all cases, the on-site levels of radioactivity or of other agents which were found in above ambient background in ground water appeared to be confined to within a hundred meters of their origin. They would require decades of travel before reaching the site boundary. Concentrations of radioactivity, and water quality parameters, in ground water from perimeter surveillance we $11 \mathrm{~s}$ (other than those adjacent to the Peconic River) were at or near background and only a few percent of the EPA Drinking Water Standards (18).

A study conducted in 1979, with the collaboration of the Suffolk County Department of Health Services (SCDHS), on the distribution of ${ }^{90} \mathrm{Sr}$ in survei1lance wells throughout the county wells indicated that they were related to depth of water table. The largest concentrations, in four of 43 samples, ranged from 1.1 to $2.75 \mathrm{pCi} 1^{-1}$. There was no apparent correlation between the presence of $90 \mathrm{Sr}$ in these wells and their proximity to the Laboratory. In the remaining wells the concentrations of ${ }^{90} \mathrm{Sr}$ were below $0.5 \mathrm{pCi} 1^{-1}$.

The collective average dose equivalent rate (total population dose) attributable to Laboratory sources, for the population up to a distance of 80 $\mathrm{km}$, was calculated to be $5.46 \mathrm{rem} \mathrm{a}^{-1}$ (person-rem $\mathrm{a}^{-1}$ ), as compared to a natural background dose equivalent rate to the same population of about $278,405 \mathrm{rem}^{-1}$ (person-iem $a^{-1}$ ).

\subsection{MONITORING DATA COLLECTION, ANALYSIS AND EVALUATION}

\subsection{Externa1 Radiation Moniţoring;}

Dose equivalent rates at the site boundary, including natural background (as influenced by fallout) and the increments attributable to Laboratory activity, were routinely measured by the use of $\mathrm{CaF}_{2}:$ Dy thermoluminescent dosimeters (TLD) exposed for monthly periods at each of the four perimeter monitoring stations $\overline{\mathrm{P}}-2, \overline{\mathrm{P}}-4, \mathrm{P}-\overline{7}$, and $\mathrm{P}-9$, as shown in Figure 2 .

The observed weekly average dose equivalent rates for external radiation resulting from gamma activity only $(9,10)$ are given in Table 2 . There was no measurable addition to the natural background attributable to Laboratory activities, except at the northeast perimeter. At this location, the Ecology Forest irradiation source, which contained about 5883 curies $\left(2.18 \times 10^{14} \mathrm{~Bq}\right)$ of ${ }^{137} \mathrm{Cs}$ (as of $1 / 1 / 79$ ), produced a dose equivalent rate of $1.43 \pm .08 \mathrm{mRem} \mathrm{a}^{-1}$ $\left(1.43 \times 10^{-5} \pm 8 \times 10^{-7} \mathrm{~Sv} \mathrm{a}^{-1}\right)$ or $0.4 \%$ of the Radiation Protection Standard for a hypothetical individual member of the general public at this location on the Laboratory perimeter. The average external background radiation level was 57.7 mRem $a^{-1}\left(5.77 \times 10^{-4} \mathrm{~Sv} a^{-1}\right)$. As of September 28, 1979, the Ecology Forest 
TABLE 2

1979 BNL Environmental Monitoring

Weekly External Dose Equivalent Rates from Backeround and BNL Operations (mRem/week)

\begin{tabular}{|c|c|c|c|c|c|c|}
\hline Month & $P-2$ & $P-4$ & $\mathrm{P}-7$ & $\begin{array}{l}\text { Northeast } \\
\text { Perimeter } \\
\text { P-9 }\end{array}$ & Source $^{a}$ & $\begin{array}{l}\text { Average } \\
\text { Background }\end{array}$ \\
\hline January/February & 1.05 & 1.08 & 1.08 & 1.06 & $<0.00$ & 1.07 \\
\hline March & 1.14 & 1.13 & 1.13 & 1.09 & $<0.00$ & 1.13 \\
\hline April & 1.15 & 1.18 & 1.24 & 1.19 & $<0.00$ & 1.19 \\
\hline May & 1.09 & 1.12 & 1.12 & 1.14 & 0.03 & 1.11 \\
\hline June & 1.05 & 1.14 & 1.14 & 1.18 & 0.07 & 1.11 \\
\hline July & 1.17 & 1.19 & 1.18 & 1.27 & 0.09 & 1.18 \\
\hline August & 1.13 & 1.20 & 1.20 & 1.27 & 0.09 & 1.18 \\
\hline September & 1.26 & 1.29 & 1.30 & 1.31 & 0.03 & 1.28 \\
\hline October & 1.12 & $1.13^{\circ}$ & 1.16 & 1.09 & $<0.00$ & 1.14 \\
\hline November & 1.28 & 1.30 & 1.28 & 1.25 & $<0.00$ & 1.29 \\
\hline December & 1.04 & 1.13 & 1.11 & 1.09 & $<0.00$ & 1.09 \\
\hline $\begin{array}{r}\text { Total (mRem/year) } \\
\text { (50 weeks) }\end{array}$ & 56.42 & 58.26 & 58.51 & 58.53 & 1.43 & 57.74 \\
\hline Average (mRem/week) & 1.13 & 1.17 & 1.18 & 1.18 & 0.03 & 1.16 \\
\hline $\begin{array}{l}\text { Standard deviation } \\
\qquad(2 \sigma)\end{array}$ & 0.16 & 0.14 & 0.14 & 0.18 & 0.08 & 0.14 \\
\hline
\end{tabular}

Locations of monitoring stations indicated on Figure 2

a 137

Cs Ecology Forest Irradiation Source radiation level derived by subtracting average background at other stations from total measured level at northeast perimeter. This source has been decommissioned as of September 28, 1979 and was removed from the Ecology Forest area on October $29,1979$.

b Average of $\mathrm{P}-2, \mathrm{P}-4$ and $\mathrm{P}-7$, unaffected by $\mathrm{BNL}$ on site radiations or efflilents.

Data for this table supplied by J. Gilmartin (S\&EP) using CaF 2 (Dy) environmental monitoring TLDs which were placed in the above locations by E. Hartmann (S\&EP).

mRem $=0.00001 \mathrm{~Sv}$. 
source was decommissioned. It was removed from the Ecology Forest area on October 29, 1979. The TLD readings at the northeast perimeter (P-9), during the last quarter of the year, confirm the absence of the source. It is to be noted that the source radiation (photons) were less penetrating during winter months relative to the summer months as the density of air increases with the drop in temperature during winter $(9,10)$, which accounts for the increases in radiation levels noted at the northeast perimeter during the sumer months.

\subsection{Airborne Effluents and Ground-Level Air Particulates, Tritium and Radioiodine Monitoring:}

\subsubsection{Facilities and Effluents}

The principal Laboratory facilities which currently discharge radioactive effluents to the atmosphere are listed in Table 3 . The installed on-line effluent monitoring and sampling devices are also indicated. The location of these facilities on the Laboratory site are shown in Figure 2. The types and amounts of these effluents released during 1979 are shown in Table 4.

Oxygen-15 (150), Argon-41 ( $\left.{ }^{41} \mathrm{Ar}\right)$ and Xenon-127 (127 Xe) are radioactive gases. Since they have relatively short half-lives, they have the potential of being environmentally significant as sources of increased external radiation only at or relatively near the point of release. ${ }^{127} \mathrm{Xe}$ is produced at the BLIP facility for commercial use. 150 , which has a half-life of two minutes, is produced by the interaction of protons and water in the BLIP facility and evolved at an estimated rate of $0.21 \mathrm{Ci}_{\mathrm{AA}}^{-1} \mathrm{~h}^{-1}\left(7.8 \times 10^{9} \mathrm{~Bq} \mu \mathrm{A}^{-1} \mathrm{~h}^{-1}\right)$. When this facility is operated at the full beam current of $180 \mu \mathrm{A}$ the equilibrium activity of $15_{0}$ at the point of generation is $1.8 \mathrm{Ci}\left(6.6 \times 10^{10} \mathrm{~Bq}\right)$. Argon-41, which has a half-life of 110 minutes, is produced by the interaction of neutrons and ventilating air in the Medical Reactor and released form its stack at a rate of $1 \mathrm{Ci} \mathrm{MW}(\mathrm{th})^{-1} \mathrm{~h}^{-1}\left(3.7 \times 10^{10} \mathrm{~Bq} \mathrm{MW}(\mathrm{th})^{-1} \mathrm{~h}^{-1}\right)$ when it is operated at full power of $3 \mathrm{MW}(\mathrm{th})$. Assuming equilibrium is attained, a conservative assumption, the equilibrium activity is $8 \mathrm{Ci}\left(2.96 \times 10^{11} \mathrm{~Bq}\right)$ at the reactor stack. In reviewing the airborne effluent data (Table 4) over the past six years (19741979 ), it is to be observed that, except for ${ }^{12 \%} \mathrm{Xe}$, the radioactive gases released are a function of operational time and power level of the facility. Two factors have served to reduce the quantities of radioactivity released to the atmosphere from BNL facilities. At the Hot Laboratory complex (B1dg. 801), an experiment which utilized about $1000 \mathrm{Ci}\left(3.7 \times 10^{10} \mathrm{~Bq}\right.$ ) of tritium (as vapor) was discontinued during 1979. This has reduced the release of tritium from this complex by 50 times when compared with the releases during the operation of the experiment in 1978. The decrease in tritium gas released at the Van de Graaf facility from $1263 \mathrm{Ci}\left(4.67 \times 10^{13} \mathrm{~Bq}\right)$ in 1978 to $80 \mathrm{Ci}\left(2.96 \times 10^{12} \mathrm{~Bq}\right)$ in 1979 is the result of improved decontamination techniques such as scrubbing which has reduced the quantities released to the environment. A second order effect which has contributed to the decrease is the accurate calibration of the Kanne chamber resulting in the increased reliability of data collection and improving the confidence in the reported tritium releases. 
1979 BNL Environmental Monitoring Gaseous Effluent

Release Locations and On-Line Monitoring and Sampling Devices

\begin{tabular}{|c|c|c|c|c|c|}
\hline Buildjng & $\begin{array}{l}\text { Facility and } \\
\text { release point } \\
\text { radioactive effluents }\end{array}$ & $\begin{array}{l}\text { Release } \\
\text { height } \\
\text { (m) }\end{array}$ & $\begin{array}{l}\text { Principal } \\
\text { nuclide (s) }\end{array}$ & $\begin{array}{l}\text { On-Line } \\
\text { monitoring }\end{array}$ & $\begin{array}{l}\text { Sampling } \\
\text { devices } \\
\end{array}$ \\
\hline 490 & $\begin{array}{l}\text { Medical Research Center } \\
\text { Roof Stack }\end{array}$ & 13.7 & Tritium (vapor) & None & $\begin{array}{l}\text { Dessicant for } \\
\text { tritium vapor }\end{array}$ \\
\hline 491 & Medical Research Reactor & 45.7 & Argon -41 & $\begin{array}{l}\text { Moving tape for } \\
\text { radio-particulates }\end{array}$ & $\begin{array}{l}\text { Charcoal for } \\
\text { radioiodines }\end{array}$ \\
\hline 555 & Chemistry Building Roof & 16.8 & Tritium (vapor) & None & $\begin{array}{l}\text { Dessicant for } \\
\text { tritiam vapor }\end{array}$ \\
\hline $\begin{array}{l}750 \\
801\end{array}$ & $\begin{array}{l}\text { High Flux Beam Reactor/ } \\
\text { Hot Laboratory Stack }\end{array}$ & 97.5 & Tritium (vapor) & $\begin{array}{l}\text { Beta scintillator for } \\
\text { radioactive gases; } \\
\text { Kanne chamber for } \\
\text { tritium (gas+vapor) }\end{array}$ & $\begin{array}{l}\text { Dessicant for } \\
\text { tritium vapor; } \\
\text { particulate } \\
\text { filter for gross } \\
\text { beta; charcoal } \\
\text { cartridge for } \\
\text { radioiodines }\end{array}$ \\
\hline 901 & Van de Graaff Accelerator & 18.3 & $\begin{array}{l}\text { Tritium (gast } \\
\text { vapor) }\end{array}$ & $\begin{array}{l}\text { Kanne chamber for } \\
\text { tritium (gas+vapor) }\end{array}$ & $\begin{array}{l}\text { Dessicant for } \\
\text { tritium vapor }\end{array}$ \\
\hline 931 & Linac Isotope Facility & 18.3 & $\begin{array}{l}\text { Tritium (vapor) } \\
\text { Oxygen-15 }\end{array}$ & $\begin{array}{l}\text { G-M detector for } \\
\text { radiogases }\end{array}$ & $\begin{array}{l}\text { Dessicant for } \\
\text { tritium vapor }\end{array}$ \\
\hline \multicolumn{6}{|c|}{ Steam Plant Effluents } \\
\hline 610 & Cəntral Steam Plant Stack & 19.8 & $\begin{array}{l}\text { Particulates; } \\
\mathrm{SO}_{2} ; \mathrm{NO}_{\mathrm{x}}\end{array}$ & None & None \\
\hline
\end{tabular}

\footnotetext{
${ }^{a}$ Locations given in Figare 2
} 
TABLE 4

1979 BNL Environmental Monitoring Airborne Effluent Data Radioactive Effluents

\begin{tabular}{|c|c|c|c|c|}
\hline Building & Facility and release point & $\begin{array}{l}\text { Elevation }{ }^{a} \\
(\mathrm{~m})\end{array}$ & Nuclide & $\begin{array}{l}\text { Activity } \\
\text { released }(\mathrm{Ci})\end{array}$ \\
\hline 491 & $\begin{array}{l}\text { Medical Research Reactor } \\
\text { Stack }\end{array}$ & 45.7 & ${ }^{41} \mathrm{Ar}$ & $314.9^{\mathrm{b}}$ \\
\hline 190 & $\begin{array}{l}\text { Medical Roocaroh Conter } \\
\text { Stack }\end{array}$ & 13.7 & ${ }^{3} \mathrm{H}$ (vapor) & 1.8 \\
\hline 555 & Chemistry Building Stack & 16.8 & ${ }^{3} \mathrm{H}\left(\mathrm{Vug}_{\mathrm{g}} \underline{\mathrm{L}}\right)$ & 15.3 \\
\hline 750 & High Flux Beam Reactor & 97.5 & ${ }^{3} \mathrm{H}$ (vapor) & 119.2 \\
\hline \multirow[t]{4}{*}{801} & Hot Laboratory Stack & & ${ }^{3} \mathrm{H}$ (vapor) & $0.8 \times 10^{-3}$ \\
\hline & & & $\begin{array}{l}\text { Gross Beta } \\
\text { (Harticulatè) }\end{array}$ & $1.7 \times 10^{-4}$ \\
\hline & . & . & $123 \mathrm{Xe}$ & 0.6 \\
\hline & & & $127 \mathrm{Xe}$ & 1.0 \\
\hline \multirow[t]{2}{*}{901} & Van de Graaff Accelerator & 18.3 & $3_{\mathrm{H}}$ (gas) & 79.5 \\
\hline & . & & $3_{H}$ (vapor) & 9,3 \\
\hline \multirow[t]{2}{*}{931} & Iinac Isotope Production & 18.3 & $3_{H}$ (vapor) & $24.7 \times 10^{-3}$ \\
\hline & Facility & & $15_{0}$ & $27483^{\circ}$ \\
\hline
\end{tabular}

Above ground level.

b Calculated from reported operating time and "one-time" measured emission race at $3 \mathrm{MW}$ power level.

c Calculated from reported operating time and estimated production rate at 180 uamp full beam current.

$\mathrm{Ci}=3.7 \times 10^{10} \mathrm{~Bq}$. 
The slight increase in tritium (as vapor) released from the HFBR resulted principally from the purging of the heat exchanger system, preparatory for modifications to increase the power level of the facility from $40 \mathrm{MW}$ to $60 \mathrm{MW}$.

Considerable dilution with the ambient air occurs between the point of generation and release of these sources of radioactivity and the site boundary. Additionally, radioactive decay decreases the air activity concentrations of shorter lived radionuclides during the transit time between the source and the site boundary. After dilution and decay, concentrations of airborne radioactivity at the site boundary was reduced to such a level that they produced no detectable increase in the dose equivalent rate (as resulting from the discharge of radioactive effluents to the atmosphere - Table 3) during 1979.

Tritium $\left({ }^{3} \mathrm{H}\right)$ has a half-life of 12.3 years, and is a very low energy beta emitter $\left(\mathrm{T}_{\beta}(\max )=18.6 \mathrm{KeV}\right)$. Its principal environmental significance is as tritiated water vapor (HTO), when it is taken up and utilized by living systems as water. Of the $225 \mathrm{Ci}\left(8.4 \times 10^{12} \mathrm{~Bq}\right)$ of tritium released from the Laboratory facilities during $1979,79.5 \mathrm{Ci}\left(2.96 \times 10^{12} \mathrm{~Bq}\right)(35 \%)$ was in gaseous form, and $145.5 \mathrm{Ci}\left(5.4 \times 10^{12} \mathrm{~Bq}\right)(65 \%)$ was released as tritiated water vapor (HTO). As Low as Reasonably Achievable (ALARA) practices have reduced HTO releases from the HFBR by $70 \%$ since 1974 . These include replacing a portion of the heavy water (moderator and coolant) at frequent intervals (once a year) and an effective program to detect tritium and prevent its leak.

The amounts of conventional pollutants released from the central steam plant are shown in Table 5. Those for sulfur dioxide $\left(\mathrm{SO}_{2}\right)$ and nitrogen oxides $\left(\mathrm{NO}_{\mathrm{x}}\right)$ are estimated from reported emission factors for comparable plants (11), supplemented by analysis for sulfur content of the fuel oil utilized at the plant. The amount of particulates was based on the average concentration found in stack sampling of the steam boiler units in a series of tests conducted during 1977 by an Environmental Protection Agency (EPA) approved laboratory. Their results indicate the average emission rate of particulates, $0.0781 \mathrm{~b} \mathrm{MBTU} \mathrm{MU}^{-1}$, was below the emission limit of 0.1 Ib MBTU-1 as set forth by the New York State Department of Environmental Conservation (Part 227, Stationary Combustion Installations).

A review of the stack emission data over the past six years (1974-1979) has indicated reductions in $\mathrm{SO}_{2}, \mathrm{NO}_{x}$ and particulates. This is especially noticeable since 1976 when the Laboratory initiated the utilization of alternate liquid fuels (ALF), such as mineral spirits, alcohol, jet fuel and reconstituted fuels. The amount of ALF relative to total fuel consumption at the steam plant has increased from zero in 1976 to $6 \%$ in $1977,23 \%$ in 1978 and $49 \%$ in 1979 . These alternate fuels typically have a weighted average sulfur content of $0.5 \%$ or less, as compared to the typical $1 \%$ sulfur content of the $\$$ 非 oil. Thus, though the total volume of fuel consumed has gone up, the amount of fuel, if weighted to $1 \%$ sulfur content, has been reduced by $32 \%$ which, in turn, is reflected in the reduction of pollutants. Because of the uncertainties in the potential releases to the environment resulting from the combustion of these fuels, 28 samples of these alternate fuels were analyzed for cadmium (Cd) and lead $(\mathrm{Pb})$. The results, tabulated below indicate that the burning of ALF does not constitute a health hazard. 


\section{TABLE 5}

1979 BNL Environmental Monitoring Emission of $\mathrm{SO}_{2}, \mathrm{NO}_{2}$ and Part:cillates from Central Steam Plant (Bldg. 510)

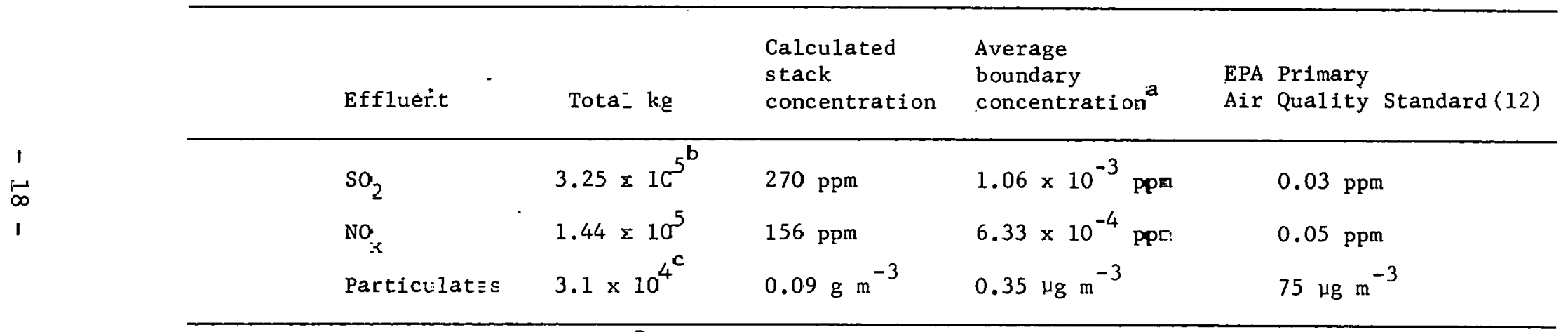

$\varepsilon_{\text {Based on average } X / Q}$ :f $2.4 \times 10^{-7}$ sez $\mathrm{m}^{-3}$ calculated by BNL Meteorology Group (1979).

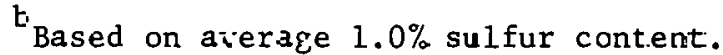

${ }^{\mathrm{C}}$ Based on masured avexage value curing February 1977 stack sampling cond scted on main steam boiler unit (New York Testing Labsratories, Inc., Westtury, N.Y., 11590).

Table based on data suppiied by Pllant Engineering (E.E. Shelton). 
Lead $(\mathrm{Pb})$

Min imum

$<1$

273

$1.04 \times 10^{-3}$

$<0.38 \times 10^{-6}$

$1.52 \times 10^{-5}$

b) if burned at the maximum possible mixture $50 \% \mathrm{ALF} / 50 \%$ 非 6

$<1.9 \times 10^{-6}$

$0.52 \times 10^{-3}$

$<0.19 \times 10^{-6}$

$0.76 \times 10^{-5}$

Standard $\left(\mu \mathrm{g} \mathrm{m}^{-3}\right)$

$1.5 *$

$39,000 * *$

**British Standard, no U.S. standard as yet promulgated.

As indicated in the 1978 Environmental Monitoring Report and as estimated from the fuel consumed in 1979 , the mercury ( $\mathrm{Hg}$ ) concentration in ALF samples was approximately 100 times below the EPA 1 imit of $4.65 \mathrm{~kg} \mathrm{day}^{-1}$ (12). An ana1ysis for chlorinated hydrocarbons was negative although small traces of chlorine were detected. Additionally, the resulting low ash content ( $<20 \mathrm{ppm}$ ) after burning ALF indicates negligible quantities of trace metals which has been confirmed by our analysis for $\mathrm{Cd}$ and $\mathrm{Pb}$. Therefore, even if the most liberal estimates of usage are employed, the environmental consequences of burning these alternate fuels with 非 fuel oil are not significant.

\subsubsection{Sampling and Analysis}

The Brookhaven environmental monitoring air sampling program is designed to i=dentify airborne radioactivity attributable to natural sources, to activities remote from the Laboratory (e.g., above ground nuclear weapons tests) and to Laboratory activities. Most of the air concentrations of radioactivity dctected during 1979 could be attributable to the first two sources. Fallout from the Chinese nuclear test which took place on December 14, 1978 was also detected.

\subsubsection{Air Samples}

High volume (500 $1 \mathrm{~min}^{-1}$ ) positive displacement air pumps (Gast 3040) were operated at a monitoring station southeast of the solid waste management areas (Fig. 2, S -6 ), and at the northeast and the southwest perimeter stations ( $\mathrm{P}-9$ and $\mathrm{P}-4)$. The air sampling media consisted of a $7.6 \mathrm{~cm}$ diameter air particulate filter (Gelman type G) followed by a $7.6 \mathrm{~cm} \times 2.5 \mathrm{~cm}$ bed of petroleumbased charcoal (Columbia Grade LC $12 / 28 \mathrm{x}$ mesh) for collection of radiohalogens. In parallel to this system is a $7.6 \mathrm{~cm}$ diameter air particulate filter (Gelman type G) followed by a $250 \mathrm{~cm}^{3}$ impregnated charcoal filter and sampled at a flow 
rate of two $\mathrm{ft}^{3} \mathrm{~min}^{-1}$. The rationale for this latter sampling system is to assure the collection of all species of radioiodines at a suitable flow rate. Short term fluctuations in airborne radioactive particulate concentrations are generally indicative of the presence of recent weapons tests debris. To distinguish between debris from nuclear weapon tests and that which might result from activities of the waste management operations, the air particulate filters at station S-6 were changed and counted on a daily basis during the work week. The air particulate filters at the other stations were changed and counted on a week 1 y basis.

After allowing several days ( $>$ seven days) for the decay of short lived natural radioactivity, the air particulate samples from the solid waste management area station were analyzed for gross alpha activity using a $12.7 \mathrm{~cm}$ diameter $\mathrm{Zn}-\mathrm{S}$ coated detector optically coupled to a photomultiplier tube, and the air particulate samples from all locations were analyzed for gross beta activity using a $12.7 \mathrm{~cm}$ beta scintillator. These data are shown in Table 6 . This table also includes data from a source point--the HFBR stack. The sampling point is located in the stack in such a manner that it samples the exhaust air from the Hot Lab (BIdg. 801) and the HFBR after it passes through absolute filters.. The data, therefore, represent the concentration at the HFBR stack. The seasonal trend of an early spring maximum, as observed for both gross alpha and gross beta activity in 1975, shifted toward late spring in 1976, early sumer in 1977, and for 1978 and 1979 has returned to late spring and early summer maximum. An increase in gross beta and alpha activity observed during previous years has been slightly decreased. In general, the gross beta activity at the Waste Management area was about three times that of the SW and NE Perimeter areas, the latter two being usually similar in value. These differences indicate the presence of Laboratory-produced radionuclides in air particulate samples. However, the gross beta activity at all monitoring stations had shown a significant increase in March 1978 and to a slight extent in December 1978 following the Chinese nuclear tests. This increase was not seen in the early part of 1979 indicating the rapid dispersal of fallout activity.

In addition to the daily and/or weekly gross beta counts indicated above, analyses for gamma emitting nuclides were performed on a monthly composite of all individual air particulate samples shortly after the end of each month. The charcoal samples were analyzed at one month post-collection to determine ${ }^{131}$ I by decay in its full energy peak region during this time. These data are reported in Table 7. Gross beta activity in air that had increased following the Chinese nuclear test in late 1976 began to decline in early 1977 with a slight increase in 1978 due to scavenging by heavy precipitation. The decline continued in 1979. When compared to data from 1976 to 1979 , it seems that there is evidence of an early or late spring maximum, which for 1979 indicates a late arrival of the maximum. The increase in December 1978 following the Chinese nuclear test did not follow through in early 1979. Fission product nuclides such as $103_{\mathrm{Ru}}$, ${ }^{106} \mathrm{Ru},{ }^{137} \mathrm{Cs},{ }^{141} \mathrm{Ce}$, and ${ }^{144} \mathrm{Ce}$ were at or below Minimum Detection Limit (MDL) for the counting system used (Table 29). Although statistically significant levels of 131 I were noted during the earlier Chinese nuclear tests in 1976 and 1977 it was not detected during 1979. Naturally occurring ${ }^{3}$ Be was present in low but near uniform concentration throughout the year. These data indicate 
Table 6

1979 BNL ENV!RONMENTAL MONITORING AVERAGE GROSS ALPHA.

AND GROSS BETA CONCENTRATIONS, AIR PARTICULATE FILTERS (PCI/M3 OR 1.03E-12 UCI/CM3)

- NO. AVERA

\begin{tabular}{|c|c|c|c|c|c|c|c|c|c|c|}
\hline & LOCATION & No. & $\begin{array}{l}\text { AL. } \\
\text { AVERAGE }\end{array}$ & $\begin{array}{l}\text { PHA } \\
\text { MAXIMUM }\end{array}$ & MINIMUM & No. & $\begin{array}{l}\text { BETA } \\
\text { AVERAGE }\end{array}$ & MAXIMUM & MINIMUM & IL FLOW(M3) \\
\hline JANUARY & $\begin{array}{l}\text { WASTE AREA } \\
\text { S.W. PERIM } \\
\text { N.E. PERIM } \\
\text { STACK }\end{array}$ & 22 & .0010 & .0032 & .0001 & $\begin{array}{r}22 \\
5 \\
4 \\
5\end{array}$ & $\begin{array}{l}.7703 \\
.0589 \\
.0701 \\
.0520\end{array}$ & $\begin{array}{r}.1610 \\
.1050 \\
.1120 \\
1.0000\end{array}$ & $\begin{array}{l}.0254 \\
.0182 \\
.0479 \\
.0427\end{array}$ & $\begin{array}{l}2.100 E+04 \\
1.753 E+04 \\
1.4 E 1 E+04 \\
9.049 E+02\end{array}$ \\
\hline FEBRUARY & $\begin{array}{l}\text { WASTE AREA } \\
\text { S.W. PERIM } \\
\text { N.E. PERIM } \\
\text { STACK }\end{array}$ & 19 & .0013 & .0027 & .0001 & $\begin{array}{r}19 \\
4 \\
3 \\
4\end{array}$ & $\begin{array}{l}.0692 \\
.0558 \\
.0695 \\
.2965\end{array}$ & $\begin{array}{l}.1350 \\
.0668 \\
.0721 \\
.8620\end{array}$ & $\begin{array}{l}.0229 \\
.0257 \\
.0667 \\
.0526\end{array}$ & $\begin{array}{l}1.807 E+04 \\
1.458 E+04 \\
8.831 E+03 \\
7.245 E+02\end{array}$ \\
\hline MARCH & $\begin{array}{l}\text { WASTE AREA } \\
\text { S.W. PERIM } \\
\text { N.E. PERIM } \\
\text { STACK }\end{array}$ & 22 & .0010 & .0025 & .0002 & $\begin{array}{r}22 \\
4 \\
4 \\
4\end{array}$ & $\begin{array}{l}.0708 \\
.0558 \\
.0530 \\
.0420\end{array}$ & $\begin{array}{l}.2070 \\
.0727 \\
.0842 \\
.0548\end{array}$ & $\begin{array}{l}.0351 \\
.0416 \\
.0294 \\
.0270\end{array}$ & $\begin{array}{l}1.728 E+04 \\
1.258 E+04 \\
1.619 E+04 \\
7.121 E+02\end{array}$ \\
\hline APRIL & $\begin{array}{l}\text { WASTE AREA } \\
\text { S.W. PERIM } \\
\text { N.E. PERIM } \\
\text { STACK }\end{array}$ & 19 & .0009 & .0019 & .0000 & $\begin{array}{r}19 \\
5 \\
5 \\
5\end{array}$ & $\begin{array}{l}.1481 \\
.0614 \\
.0980 \\
.1735\end{array}$ & $\begin{array}{l}.4110 \\
.1540 \\
.1620 \\
.5680\end{array}$ & $\begin{array}{l}.0632 \\
.0040 \\
.0490 \\
.0289\end{array}$ & $\begin{array}{l}2.284 E+04 \\
1.451 E+04 \\
1.203 E+04 \\
7.818 E+02\end{array}$ \\
\hline BAY & $\begin{array}{l}\text { WASTE AFEA } \\
\text { S.W. PERIM } \\
\text { N.E. PERIM } \\
\text { STACK }\end{array}$ & ¿2 & .0006 & .0017 & . Guบũ & $\begin{array}{r}22 \\
4 \\
4 \\
4\end{array}$ & $\begin{array}{l}.1965 \\
.1087 \\
.1210 \\
.0393\end{array}$ & $\begin{array}{l}.4900 \\
.1280 \\
.1430 \\
.4430\end{array}$ & $\begin{array}{l}.0854 \\
.0692 \\
.0825 \\
.0208\end{array}$ & $\begin{array}{l}1.762 E+04 \\
1.624 E+04 \\
1.366 E+04 \\
6.545 E+02\end{array}$ \\
\hline JUNE & $\begin{array}{l}\text { WASTE AREA } \\
\text { S.W. PERIM } \\
\text { N.E. PERIM } \\
\text { STACK }\end{array}$ & 20 & .0005 & .0019 & .0000 & $\begin{array}{r}20 \\
4 \\
4 \\
4\end{array}$ & $\begin{array}{l}.1853 \\
.1188 \\
.1276 \\
.0282\end{array}$ & $\begin{array}{l}.4120 \\
.1540 \\
.1670 \\
.0345\end{array}$ & $\begin{array}{l}.0956 \\
.0943 \\
.0975 \\
.0113\end{array}$ & $\begin{array}{l}1.660 E+04 \\
1.583 E+04 \\
1.380 E+04 \\
4.563 E+03\end{array}$ \\
\hline JULY & $\begin{array}{l}\text { WASTE AREA } \\
\text { S.W. PERIM } \\
\text { N.E. PERIM } \\
\text { STACK }\end{array}$ & 18 & .0007 & .0017 & .0001 & $\begin{array}{r}18 \\
8 \\
5 \\
5\end{array}$ & $\begin{array}{l}.1628 \\
.1156 \\
.1319 \\
.0735\end{array}$ & $\begin{array}{l}.2960 \\
.2100 \\
.2270 \\
.1420\end{array}$ & $\begin{array}{l}.0865 \\
.0918 \\
.0835 \\
.0209\end{array}$ & $\begin{array}{l}1.602 E+04 \\
1.612 E+04 \\
1.298 E+04 \\
8.412 E+02\end{array}$ \\
\hline AUGUST & $\begin{array}{l}\text { WASTE AREA } \\
\text { S.W. PERIM } \\
\text { N.E. PERIM } \\
\text { STACK }\end{array}$ & 23 & .0040 & .1010 & .0002 & $\begin{array}{r}23 \\
4 \\
4 \\
5\end{array}$ & $\begin{array}{l}.2106 \\
.0944 \\
.0838 \\
.0547\end{array}$ & $\begin{array}{l}.5930 \\
.1120 \\
.0944 \\
.0865\end{array}$ & $\begin{array}{l}.0106 \\
.0785 \\
.0730 \\
. C=19\end{array}$ & $\begin{array}{l}1.774 E+04 \\
1.317 E+04 \\
1.437 E+04 \\
9.323 E+02\end{array}$ \\
\hline SEPTEMBER & $\begin{array}{l}\text { WASIE AREA } \\
\text { S.W. PER!M } \\
\text { N.E. PERIM } \\
\text { STACK }\end{array}$ & 13 & .0006 & .0013 & .0001 & $\begin{array}{r}13 \\
4 \\
4 \\
4\end{array}$ & $\begin{array}{l}.7640 \\
.0717 \\
.0733 \\
.1494\end{array}$ & $\begin{array}{l}.7600 \\
.0852 \\
.0970 \\
.3470\end{array}$ & $\begin{array}{l}.0976 \\
.0634 \\
.0546 \\
.0213\end{array}$ & $\begin{array}{l}1.027 E+04 \\
1.303 E+04 \\
1.238 E+04 \\
6.932 E+02\end{array}$ \\
\hline OCTOBER & $\begin{array}{l}\text { WASTE AREA } \\
\text { S.W. PERIM } \\
\text { N.E. PERIM } \\
\text { SIACK }\end{array}$ & 19 & .0018 & .0309 & .0002 & $\begin{array}{r}21 \\
6 \\
5 \\
4\end{array}$ & $\begin{array}{l}.0870 \\
.0619 \\
.0600 \\
.0599\end{array}$ & $\begin{array}{l}.3190 \\
.0900 \\
.0792 \\
.0690\end{array}$ & $\begin{array}{l}.0257 \\
.0418 \\
.0451 \\
.0419\end{array}$ & $\begin{array}{l}1.815 E+04 \\
1.489 E+04 \\
1.383 E+04 \\
8.378 E+02\end{array}$ \\
\hline NOVEMBER & $\begin{array}{l}\text { WASTE AREA } \\
\text { S.W. PERIM } \\
\text { N.E. PERIM } \\
\text { STACK }\end{array}$ & 18 & .0012 & .0022 & .0003 & $\begin{array}{r}18 \\
4 \\
3 \\
4\end{array}$ & $\begin{array}{l}.1063 \\
.0839 \\
.0654 \\
.0361\end{array}$ & $\begin{array}{l}.1800 \\
.1350 \\
.1100 \\
.0565\end{array}$ & $\begin{array}{l}.0554 \\
.0575 \\
.044 ! \\
.0192\end{array}$ & $\begin{array}{l}1.664 E+04 \\
1.067 E+04 \\
1.209 E+04 \\
6.123 E+02\end{array}$ \\
\hline DECEMBER & $\begin{array}{l}\text { WASTE AREA } \\
\text { S.W. PERIM } \\
\text { N.E. PERIM } \\
\text { STACK. }\end{array}$ & 19 & .0008 & .0023 & .0002 & $\begin{array}{r}18 \\
4 \\
4 \\
3\end{array}$ & $\begin{array}{l}.1: 12 \\
.0571 \\
.0478 \\
.0229\end{array}$ & $\begin{array}{l}.3010 \\
.1260 \\
.0510 \\
.0324\end{array}$ & $\begin{array}{l}.0373 \\
.0416 \\
.0403 \\
.0041\end{array}$ & $\begin{array}{r}1.887 E+04 \\
1.357 E+04 \\
1.535 E+04 \\
4.054 E+02\end{array}$ \\
\hline YTD & $\begin{array}{l}\text { WASTE AREA } \\
\text { S.W. PERIM } \\
\text { N.E. PERIM } \\
\text { STACK }\end{array}$ & 234 & .0012 & .1010 & .0000 & $\begin{array}{r}235 \\
56 \\
49 \\
5 !\end{array}$ & $\begin{array}{l}.1340 \\
.0794 \\
.0828 \\
.08+5\end{array}$ & $\begin{array}{r}.7600 \\
.2100 \\
.2270 \\
1.0000\end{array}$ & $\begin{array}{l}.0105 \\
.0040 \\
.0294 \\
.0041\end{array}$ & $\begin{array}{l}\text { 2. } 119 E+05 \\
1.727 E+05 \\
1.605 E+05 \\
1.265 E+04\end{array}$ \\
\hline FIRST OTR & $\begin{array}{l}\text { WASTE AREA } \\
\text { S.W. PERIM } \\
\text { N.E. PERIM } \\
\text { STACK }\end{array}$ & 63 & .0011 & .0032 & .0001 & $\begin{array}{l}63 \\
13 \\
11 \\
13\end{array}$ & $\begin{array}{l}.0701 \\
.0570 \\
.0530 \\
.2058\end{array}$ & $\begin{array}{r}.2070 \\
.1060 \\
.1120 \\
1.0000\end{array}$ & $\begin{array}{l}.0229 \\
.0182 \\
.0294 \\
.0270\end{array}$ & $\begin{array}{l}5.634 E+04 \\
4.468 E+04 \\
3.963 E+04 \\
2.342 E+0 .\end{array}$ \\
\hline SEGHD OTR & $\begin{array}{l}\text { WASTC ARCA } \\
\text { S.W. PERIM } \\
\text { N.E. PERIM } \\
\text { STACK }\end{array}$ & 61 & .0007 & . & nחnח & $\begin{array}{l}11 \\
13 \\
13 \\
13\end{array}$ & $\begin{array}{l}.1739 \\
.0974 \\
.1163 \\
.0483\end{array}$ & $\begin{array}{l}.4900 \\
.1540 \\
.1670 \\
.5680\end{array}$ & $\begin{array}{l}.0632 \\
.0040 \\
.0190 \\
.0113\end{array}$ & $\begin{array}{l}5.707 E+04 \\
4.658 E+04 \\
3.950 E+04 \\
6.000 E+03\end{array}$ \\
\hline TH!RD QTR & $\begin{array}{l}\text { WASTE AREA } \\
\text { S.W. PERIM } \\
\text { N.E. PERIM } \\
\text { STACK }\end{array}$ & 54 & .0020 & .1010 & .0001 & $\begin{array}{l}54 \\
16 \\
13 \\
14\end{array}$ & $\begin{array}{l}.2056 \\
.0955 \\
.0959 \\
.0877\end{array}$ & $\begin{array}{l}.7600 \\
.2100 \\
.2270 \\
.3470\end{array}$ & $\begin{array}{l}.0106 \\
.0634 \\
.0546 \\
.0209\end{array}$ & $\begin{array}{l}4.402 E_{2}+04 \\
4.232 E+04 \\
4.023 E+04 \\
2.467 E+03\end{array}$ \\
\hline LAST OTR & $\begin{array}{l}\text { WASTE AREA } \\
\text { S.W. PERIM } \\
\text { N.E, PERIM } \\
\text { STACK }\end{array}$ & 56 & .001 .2 & .0309 & .0002 & $\begin{array}{l}57 \\
14 \\
12 \\
11\end{array}$ & $\begin{array}{l}.1005 \\
.0662 \\
.0570 \\
.0440\end{array}$ & $\begin{array}{l}.3190 \\
.1350 \\
.1100 \\
.0690\end{array}$ & $\begin{array}{l}.0257 \\
.0416 \\
.0403 \\
.0041\end{array}$ & $\begin{array}{l}5.447 E+04 \\
3.913 E+04 \\
4.127 E+04 \\
1.856 E+03\end{array}$ \\
\hline
\end{tabular}

YTD: Yearly total

Reference Standards: Table 29 
TABLE 7

1979 BNL Environmental Monitoring Monthly Average Concentrations of Gross Beta Activity and of Gamma Emitting Nuclides in Monthly Composite Air Particulate and Charcoal Filters

$$
\left(\mathrm{pCi} / \mathrm{m}^{3} \text { or } 10^{-12} \mathrm{\mu Ci} / \mathrm{ml}\right)
$$

\begin{tabular}{|c|c|c|c|c|}
\hline \multirow[b]{2}{*}{ Month } & \multirow[b]{2}{*}{$\begin{array}{l}\text { Average } \\
\text { Croce } \beta\end{array}$} & \multirow{2}{*}{$\begin{array}{l}\text { Sample } \\
\text { Volume } \\
\left(\mathrm{m}^{3}\right)\end{array}$} & \multicolumn{2}{|c|}{ NUCLIDES } \\
\hline & & & ${ }^{7} \mathrm{BC}$ & $14 n_{\Pi_{-}-L}$ \\
\hline January & 0.066 & 53140 & - & - \\
\hline February & 0.065 & 4.1481 & .009 & - \\
\hline March & 0.060 & 46050 & .07 & - \\
\hline April & 0.110 & 49380 & .06 & $\cdot-$ \\
\hline May & 0.145 & 47520 & .10 & .003 \\
\hline June & 0.145 & 46230 & .08 & - \\
\hline July & 0.137 & 45120 & .08 & .002 \\
\hline August & 0.137 & 45280 & .09 & - \\
\hline September & 0.127 & 36180 & .06 & - \\
\hline Uctober & 0.011 & $468 / 0$ & .09 & .003 \\
\hline November & 0.088 & 39400 & .08 & .002 \\
\hline December & 0.075 & 47790 & - & - \\
\hline Average & 0.102 & & .01 & \\
\hline Radiation & & & & \\
\hline $\begin{array}{l}\text { Concentration } \\
\text { Guide [13] }\end{array}$ & 100 & & $4 \times 10^{4}$ & 1000 \\
\hline
\end{tabular}

Error of the counting of samples is estimated to be about $15 \%$.

Radionuclides such as ${ }^{131} \mathrm{I},{ }^{137} \mathrm{Cs},{ }^{144} \mathrm{Ce}$ all below MDL. (Table 29)

See Figure 2 for location of sampling stations: P-2, P-4, P-7, P-9, S-6 and S-13. $\mathrm{pCi}=3.7, \times 10^{-2} \mathrm{~Bq}$. 
the absence of Laboratory effluent contributions at concentrations 1/100,000 or less than radiation protection guide levels.

Sampling for tritium vapor was performed at the same air sampling stations by drawing a small side stream of air $\left(\backsim 100 \mathrm{~cm}^{3} \mathrm{~min}^{-1}\right)$ through silica gel cartridges which were generally changed on a monthly basis. During colder months, the sampling cycle was lengthened due to low humidity. The collected vapor was subsequently removed from the gel by heating, then condensed and assayed by liquid scintillation counting. The tritium air concentration data obtained during 1979 is shown in Table 8. The measured yearly average concentration (including background) at the site boundary, about $6.5 \mathrm{pCi} \mathrm{m}^{-3}(0.065 \mathrm{x}$ $10^{-10} \mu \mathrm{Ci} \mathrm{cm}^{-3}$ or $0.24 \times 10^{-6} \mathrm{~Bq} \mathrm{~cm}^{-3}$ ), was $0.004 \%$ of the applicable Radiation Concentration Guide (RCG).

The current Laboratory environmental monitoring program does not include air sampling for nonradioactive substances. The calculated annual average concentrations at the site boundary of the conventional pollutants released from the central steam plant are listed in Table 5. All were less than $2 \%$ of the EPA Primary Air Quality Standard for the reported constituents. As discussed earlier in this Section, the use of ALF with $\equiv_{16}$ fuel oil does not represent a significant impact on the environment.

About $250 \mathrm{~kg}$ of various pesticides, chiefly organo-phosphates, Thiodan, Diazinon, Carbaryl and Parathion, were applied on site during 1978, principally to protect crops which were grown for biological research purposes. All of these pesticides are considered biodegradable, with persistence times in the order of a week (14). Furthermore, they were applied with a "sticker" additive to minimize becoming airborne subsequently.

\subsubsection{Precipitation}

Two pot-type rain collectors each with a surface area of $0.33 \mathrm{~m}^{2}$, are situated adjacent to the sewage treatment plant (see Fig. 2). Two routine collections were made from these, one whenever precipitation was observed during a previous 24 hour (or weekend) period, and the other once a week (whether or not precipitation occurred) by washing down the rain collector with a known volume of water. Part of each collection was evaporated for gross beta counting, a sma 11 fraction was composited for monthly tritium analysis, and the balance was put through ion exchange columns for subsequent quarterly ${ }^{90} \mathrm{Sr}$ and gamma analyses. The data for 1979 (with the exception of tritium) are reported in Table 9. There was no detectable indication of Laboratory released airborne radioactivity in precipitation collected on site. The gross beta activity does reflect rainfall scavenging of radioactive fallout from the last Chinese nuclear test of December 14, 1978. The amounts of naturally produced gamma emitters, such as ${ }^{7} \mathrm{Be}$ and ${ }^{22} \mathrm{Na}$, which have been slightly higher each year since 1975 , especially during 1977 and 1978 (see EM Reports 1975-1978) were reduced by more than 50\% in 1979. Fission and activation products, such as ${ }^{54} \mathrm{Mn}, 65 \mathrm{Zn},{ }^{95} \mathrm{Zr}-\mathrm{Nb}$ and ${ }^{131} \mathrm{I}$, were all below their MDL (Table 29). 
TABLE 8

1979 BNL Environmental Monitoring Average Tritium Vapor Concentration in Air

$$
\left(\mathrm{pCi} / \mathrm{m}^{3} \text { or } 10^{-12} \mu \mathrm{Ci} / \mathrm{ml}\right)
$$

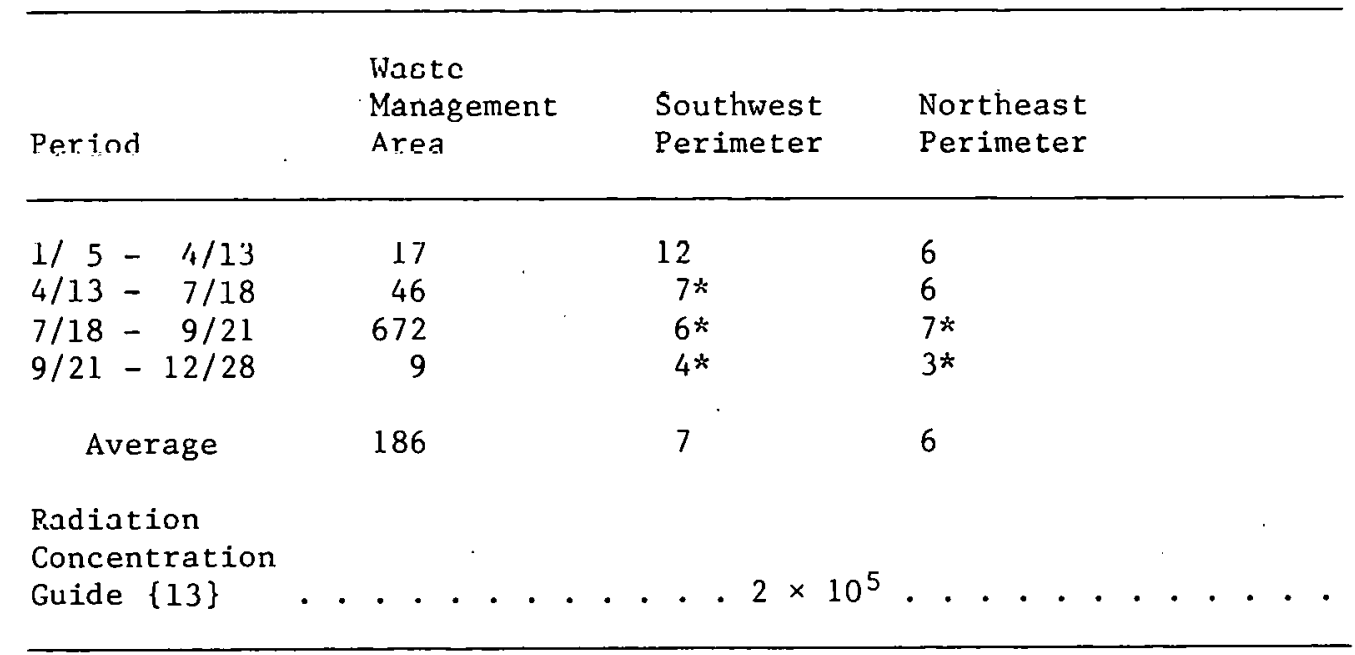

* Lega than MIDL.

$$
\begin{aligned}
& \mu \mathrm{Ci}=3.7 \times 10^{4} \mathrm{~Bq} . \\
& p C i=3.7 \times 10^{-2} \mathrm{~Bq} .
\end{aligned}
$$


TABLE 9

1979 Monthly Average Gross Beta Concentration,

Totel Gross Beta Activity and Individual Nuclide Activity in Precipitation a

\begin{tabular}{|c|c|c|c|c|c|c|c|c|c|c|}
\hline \multirow[b]{2}{*}{ Month } & \multirow{2}{*}{$\begin{array}{l}\text { Fainfall } \\
(=\mathrm{m})\end{array}$} & \multirow{2}{*}{$\begin{array}{c}\text { Average GB } \\
(\mathrm{pCi} / \operatorname{lit\in r} \\
\left.\text { or } 10^{-9} \mu C: i / \mathrm{ml}\right)\end{array}$} & \multicolumn{8}{|c|}{$\left(\mathrm{nCi} / \mathrm{m}^{2}\right)$} \\
\hline & & & $\mathrm{GB}$ & $7_{B}$ & $22 \mathrm{Na}$ & ${ }^{65} \mathrm{Zn}$ & $90 \mathrm{Sr}$ & $131_{I}$ & $137 \mathrm{Cs}$ & ${ }^{144} \mathrm{Ce}$ \\
\hline January & 33.0 & $1 . \epsilon$ & $0.53)$ & & & & & & & \\
\hline February & 13.4 & 23.6 & $3.16\}$ & 35.4 & 0.71 & - & .07 & - & 9.20 & - \\
\hline March & 9.0 & 6.2 & $0.56)$ & & & & & & & \\
\hline April & 12.6 & $13 . \Xi$ & 1.681 & & & & & & & \\
\hline May & 10.4 & $10 . c$ & $1.04\}$ & 11.6 & 0.10 & - & 0.11 & - & 0.10 & - \\
\hline June & 5.5 & $10 . \equiv$ & $0.57)$ & & & & & & & \\
\hline July & 1.5 & $45 . \varepsilon$ & 0.651 & & & & & & & \\
\hline August & 19.7 & $5 . ?$ & $1.04\}$ & 8.5 & 0.04 & - & 0.04 & - & 0.02 & - \\
\hline September & 8.1 & 3.2 & $0.2 E)$ & & & & & & & \\
\hline October & 11.6 & $9 . \epsilon$ & l.11) & & & & & & & \\
\hline November & 10.0 & 3.4 & $0.34\}$ & 7.0 & 0.05 & - & 0.04 & - & - & - \\
\hline December & 7.7 & 4.1 & 0.321 & & & & & & & \\
\hline Total & 142.5 & $(136.4)$ & 11.39 & 62.6 & 0.89 & - & .26 & - & 9.32 & - \\
\hline Average & 11.9 & 11.4 & $(11.30)$ & $(62.5)$ & $(0.90)$ & & & & & \\
\hline Average Ra & & . & & & & & & & & \\
\hline tion Guide & & $3 \times 10^{3}$ & $3 \times 10^{3}$ & $8 \times 10^{2}$ & $1 \times 10^{4}$ & $3 \times 10^{4}$ & $8 \times 10^{-1}$ & $8 \times 10^{1}$ & $5 \times 10^{3}$ & $3 \times 10^{3}$ \\
\hline
\end{tabular}

$\mathrm{nCi}=3.7 \times 10^{1} \mathrm{~Bq}$.

pCi $=3.7 \times 10^{-2} \mathrm{~Bq}$.

There were twc Chinese nuclear tests; one on March 14, 1978 and the other on December 14, 1978. Some fallout activity was detected at $\mathrm{BNL}$ as a result of the tests.

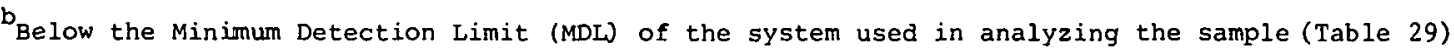


To obtain an indication of the washout of tritium from local airborne releases in addition to the pot-type collectors, small precipitation collectors were installed at the perimeter stations ( $\mathrm{P}-2, \mathrm{P}-4, \mathrm{P}-7, \mathrm{P}-9)$ and at Blue Point, some $20 \mathrm{~km}$ southwest of the Laboratory site. The average tritium concentration in the collectors located at station P-9 and at the sewage treatment plant (in the predominant downwind direction from Laboratory release locations) and at other collectors, were all reduced significantly when compared to 1974,1975 , 1976, 1977 and 1978. The levels detected were, however, at or below the MDL (Table 29). The average concentration (on site) was less than $0.01 \%$ of the RCG for drinking water. The estimated total deposition of tritium on the Laboratory site during 1979 was $<5.3 \mathrm{Ci}\left(1.96 \times 10^{11} \mathrm{~Bq}\right.$ ) (using the yearly totals of onsite and perimeter concentrations). The washout of Laboratory effluent appears to have been less than $3 \mathrm{Ci}\left(<1 \times 10^{11} \mathrm{~Bq}\right)$ or about $2 \%$ of the reported stack release of tritium vapor.

\subsection{Liquid Effluent Monitoring}

The basic principle of liquid waste management at the Laboratory is confincment and concentration in order to minimise the volumeo of liquide that would require decontamination prior to on-site release or processing into solid form for off-site burial. Accordingly, liquid wastes are segregated at the point of origin on the basis of their anticipated concentrations of radioactivity or other potentially harmful agents.

The primary water cooling systems of such facilities as the High Flux Beam Reactor, and the Medical Research Reactor, each of which contain multicurie (terabecquerel) amounts of radioactivity, are closed systems with no direct connection to any Laboratory waste system.

Small volumes (up to a few liters) of concentrated liquid wastes containing radioactivity or other hazardous agents are withheld from the Laboratory waste systems. They are stored at their sources of generation in small containers, collected by the Laboratory waste management group, and subsequently packaged for off-site disposal (in the case of hazardous agents, by an EPA licensed contractor).

Facilities which may produce larger volumes (up to several hundred liters/batch) of radioactive or otherwise contaminated waste liquids are provided with dual waste handling systems, one for "active" (D-probably contaminated) and one for "inactive" (F-probably uncontaminated) wastes. As shown in Figure 5, wastes placed into the "active" or D system are collected in holdup tanks. After sampling and analysis, they are either transferred by installed pipelines or by tank truck to storage tanks adjacent to the Laboratory 1 iquid waste evaporator. At this facility, liquids are concentrated about a hundred fold and ultimately disposed of as solid wastes. If found to be of sufficiently low concentration, $D$ wastes may be routed directly from holdup tanks to the Laboratory sanitary waste system.

As shown in Figure 5, "inactive" or $F$ wastes, depending on the results of analysis, are routed directly to the Laboratory sanitary waste system, where they are diluted by large quantities (approaching 4,000,000 $1 \mathrm{~d}^{-1}$ ) of cooling 


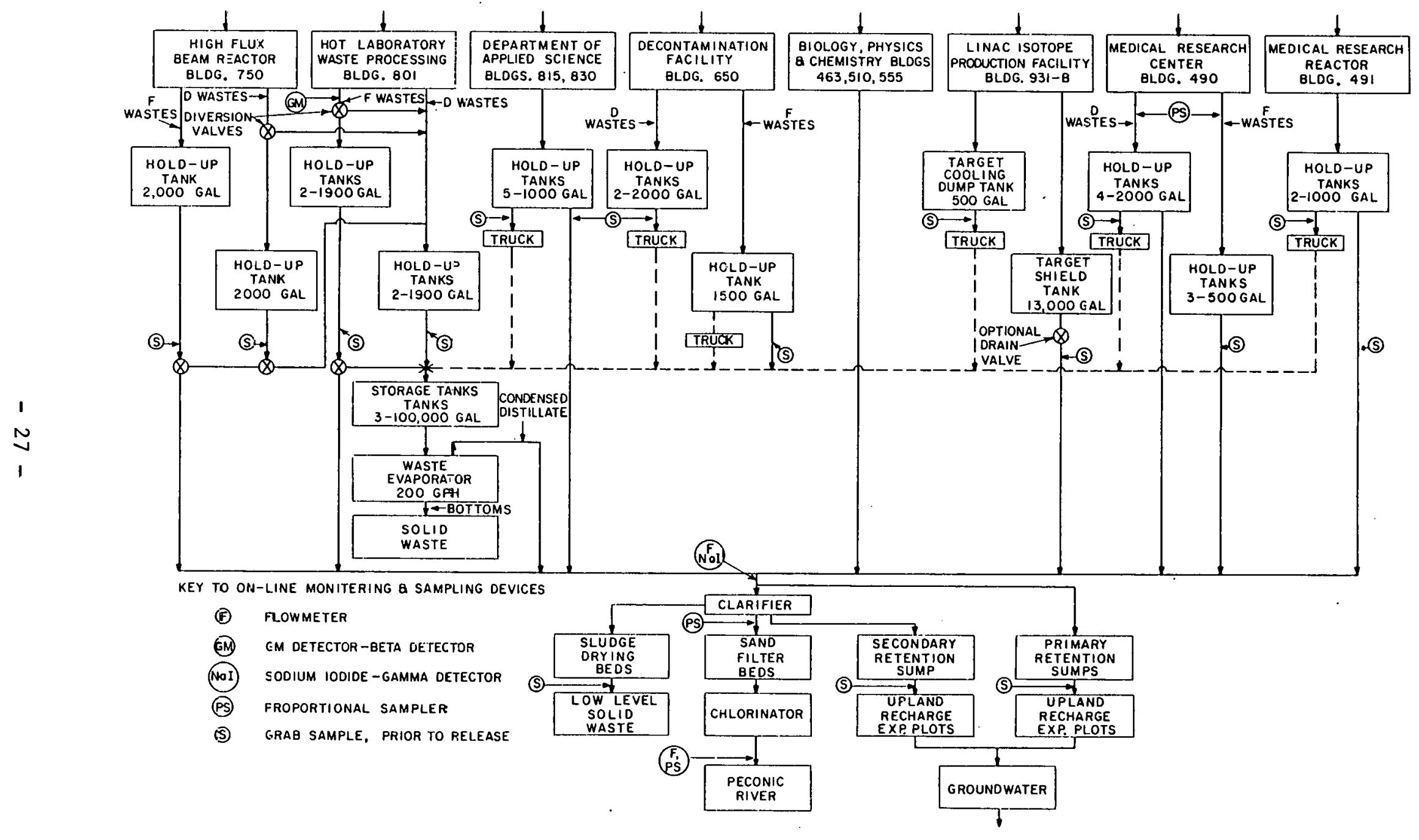

Figure 5. BNL Liquid Effluent Systems 
and other uncontaminated water routinely produced by diverse Laboratory operations. Sampling and analysis of facility holdup tanks is done to facilitate waste management; while effluent sampling is performed at the sewage treatment plant to establish the concentration and amounts of environmental releases.

The small amounts of low level radioactive waste effluents that may be routinely disposed of by release into the Laboratory sanitary waste system are established by administrative limits (15), which correspond to those applicable to sewage systems. Within these 1 imits, individual releases are kept as 1 ow as practicable.

\subsubsection{Nationa1 Pollutant Discharge Elimination System (NPDES) Permit}

As of January 31,1975 , the effluent from the Laboratory sewage treatment plant was subject to the conditions of 'l'he National Pollutant Discharge Elimination System (NPDES) Permit No. NY nกn 5835. Tharterly roporto have been pre pared in accordance with this permit using data obtained by the sewage treatment plant operators. A yearly summary of these data is shown in Table 10, which includes a specification of the permit conditions. The Laboratory effluent was within these conditions, with the exception of some daily $\mathrm{pH}$ levels which were "out of 1 imit," as set by the permit.

These pH levels were below the lower limit of 5.8 on 35 occasions. However, these were within the local natural range of ground water ( $\mathrm{pH} 4.5-5.5)$. A study has been made to determine the causative factors behind these low $\mathrm{pH}$ values. It indicates that the low $\mathrm{pH}$ of rainfall ( $\mathrm{pH} 2.5-4.9)$ on Long Island is a significant factor in lowering the $\mathrm{pH}$ of the Laboratory effluent as it passes through the sand filter beds. The U.S. EPA has been asked to consider the lowering of BNL's permit standard on this parameter ( $\mathrm{pH})$.

\subsubsection{Meadow-Marsh Project}

The Meadow-Marsh Project (16), which was conducted by the Department of Energy and Environment (DEE) since 1972 was discontinued on December 31, 1978. Since there was no effluent leaving the project sampling was also discontinued.

\subsubsection{Peconic River}

Primary treatment of the liquid stream collerted hy the sanitary wacte syslem to remove suspended solids is provided by a 950,000 1iter clarifier. The liquid effluent from it then flows onto sand filter heds, from whioh about 78\% of the water is recovered by an underlying tile field. This recovered water is chlorinated and then released into a small stream that forms one of the headwaters of the Peconic River.

A schematic of the sewage treatment plant and its related sampling arrangements are illustrated in Figure 6 . In addition to the inplant flow measurement and sampling instrumentation, totalizing flowmeters (Leopold and Stevens TP 61-2), with provision for taking a sample for each 7576 liters of flow in combination with positive action battery operated samplers (Brailsford DU-1), are 1o- 
TABLE 10

1979 BNL Environmental Monitoring National Pollution Discharge Elimination System

Summary of Data

\begin{tabular}{|c|c|c|c|c|c|c|c|c|c|c|c|c|c|}
\hline \multirow[b]{2}{*}{ Parameter } & \multirow{2}{*}{ Status } & \multicolumn{5}{|c|}{ Quantity } & \multicolumn{5}{|c|}{ Concentration } & \multirow[b]{2}{*}{$\begin{array}{l}\text { Frequency of } \\
\text { analysis }\end{array}$} & \multirow[b]{2}{*}{$\begin{array}{l}\text { Sample } \\
\text { type }\end{array}$} \\
\hline & & Minimum & Average & Maximum & Units & $\begin{array}{l}\text { No. }{ }^{a} \\
\text { ex. }\end{array}$ & Minimum & Average & Maximum & Units & $\begin{array}{l}\text { No. } \\
\text { ex. }\end{array}$ & & \\
\hline Flow & $\begin{array}{l}\text { Sample measurement } \\
\text { Permit requirement }\end{array}$ & 0.55 & 0.87 & 1.42 & MGD & - & $\begin{array}{l}\mathrm{xx} \\
\mathrm{xx}\end{array}$ & $\begin{array}{l}\mathrm{xx} \\
\mathrm{xx}\end{array}$ & $\begin{array}{l}x x \\
x x\end{array}$ & - & - & $\begin{array}{l}\text { Cont. } \\
\text { Cont. }\end{array}$ & $\begin{array}{l}\mathrm{NA} \\
\mathrm{NA}\end{array}$ \\
\hline $\mathrm{pH}$ Infiuent & $\begin{array}{l}\text { Sample measur ement } \\
\text { Permit requirement }\end{array}$ & $\begin{array}{r}6.3 \\
\mathrm{xx}\end{array}$ & $\begin{array}{l}6.8 \\
\mathrm{x} x\end{array}$ & $\begin{array}{r}9.2 \\
x x\end{array}$ & $\begin{array}{l}\text { Std. } \\
\text { Units }\end{array}$ & - & $\begin{array}{l}\mathrm{xX} \\
\mathrm{Xx}\end{array}$ & $\begin{array}{l}\mathrm{xx} \\
\mathrm{xx}\end{array}$ & $\begin{array}{l}x x \\
x x\end{array}$ & - & - & $\begin{array}{l}5 / 7 \\
\text { Daily }\end{array}$ & $\begin{array}{l}\text { Grab } \\
\text { Grab }\end{array}$ \\
\hline pH Effiuent & $\begin{array}{l}\text { Sample measur mment } \\
\text { Permit requirement }\end{array}$ & $\begin{array}{l}5.5 \\
5.8\end{array}$ & $\begin{array}{r}5.9 \\
\mathrm{xx}\end{array}$ & $\begin{array}{l}6.5 \\
9.0\end{array}$ & $\begin{array}{l}\text { Sts. } \\
\text { Units }\end{array}$ & 35 & $\begin{array}{l}\mathrm{xX} \\
\mathrm{xx}\end{array}$ & $\begin{array}{l}\mathrm{xx} \\
\mathrm{xx}\end{array}$ & $\begin{array}{l}\mathrm{xx} \\
\mathrm{xx}\end{array}$ & - & - & $\begin{array}{l}5 / 7 \\
\text { Daily }\end{array}$ & $\begin{array}{l}\text { Grab } \\
\text { Grab }\end{array}$ \\
\hline $\mathrm{BOD}_{S}$ Inflyent & $\begin{array}{l}\text { Sample measurement } \\
\text { Permit requirement }\end{array}$ & $\begin{array}{r}90.9 \\
\mathrm{XX}\end{array}$ & $\begin{array}{r}149.8 \\
x x\end{array}$ & $\begin{array}{r}218.4 \\
x x\end{array}$ & $\begin{array}{l}\mathrm{Kg} / \\
\text { day }\end{array}$ & - & $\begin{array}{r}22.0 \\
x x\end{array}$ & $\begin{array}{r}33.2 \\
\mathrm{xx}\end{array}$ & $\begin{array}{r}50.5 \\
\mathrm{xx}\end{array}$ & $\mathrm{mg} / 1$ & - & $\begin{array}{l}\text { Weekly } \\
\text { Monthly }\end{array}$ & $\begin{array}{l}8 \mathrm{hr} . \\
8 \mathrm{hr} .\end{array}$ \\
\hline $\begin{array}{c}\mathrm{BOD}_{5} \text { Effluent } \\
-\end{array}$ & $\begin{array}{l}\text { Sample measurement } \\
\text { Permit requirgment }\end{array}$ & $\begin{array}{r}6.6 \\
x \times\end{array}$ & $\begin{array}{r}13.2 \\
262.0\end{array}$ & $\begin{array}{r}24.6 \\
391.0\end{array}$ & $\begin{array}{l}\mathrm{Kg} / \\
\text { day }\end{array}$ & 0 & $\begin{array}{r}2.2 \\
\mathrm{xx}\end{array}$ & $\begin{array}{r}4.0 \\
30.0\end{array}$ & $\begin{array}{r}7.2 \\
45.0\end{array}$ & $\mathrm{mg} / 1$ & 0 & $\begin{array}{l}\text { Weekly } \\
\text { Monthly }\end{array}$ & $\begin{array}{l}8 \mathrm{hr} . \\
8 \mathrm{hr} .\end{array}$ \\
\hline $\begin{array}{l}\text { Percent removal, } \\
\text { BOD }_{5}\end{array}$ & $\begin{array}{l}\text { Sample measurament } \\
\text { Permit requirement }\end{array}$ & $\begin{array}{l}\mathrm{XX} \\
\mathrm{xx}\end{array}$ & $\begin{array}{l}\mathrm{xX} \\
\mathrm{xx}\end{array}$ & $\begin{array}{l}\mathrm{xx} \\
\mathrm{xx}\end{array}$ & - & - & $\begin{array}{r}85.1 \\
x x\end{array}$ & $\begin{array}{r}91.0 \\
x x\end{array}$ & $\begin{array}{r}95.0 \\
x x\end{array}$ & $\%$ & 0 & $\begin{array}{l}\text { Weekly } \\
\text { Monthly }\end{array}$ & $\overline{-}$ \\
\hline $\begin{array}{l}\text { Suspended solids, } \\
\text { Influent }\end{array}$ & $\begin{array}{l}\text { Sample measurement } \\
\text { Permit requirement }\end{array}$ & $\begin{array}{r}53.4 \\
x x\end{array}$ & ${ }_{\mathrm{XX}}^{125.1}$ & $\begin{array}{r}207.5 \\
x x\end{array}$ & $\begin{array}{l}\mathrm{Kg} / \\
\text { day }\end{array}$ & - & $\begin{array}{r}10.5 \\
\mathrm{xx}\end{array}$ & $\begin{array}{r}26.7 \\
x X\end{array}$ & $\begin{array}{r}53.9 \\
\mathrm{xx}\end{array}$ & $\mathrm{mg} / 1$ & - & $\begin{array}{l}\text { Biweekly } \\
\text { Monthly }\end{array}$ & $\begin{array}{l}8 \mathrm{hr} . \\
8 \mathrm{hr} .\end{array}$ \\
\hline $\begin{array}{l}\text { Suspended solids, } \\
\text { Effluent }\end{array}$ & $\begin{array}{l}\text { Sample measurement } \\
\text { Permit requirement }\end{array}$ & $\begin{array}{r}0.0 \\
\mathrm{xx}\end{array}$ & $\begin{array}{r}1.5 \\
262.0\end{array}$ & $\begin{array}{r}8.0 \\
391.0\end{array}$ & $\begin{array}{l}\mathrm{Kg} / \\
\text { day }\end{array}$ & 0 & $\begin{array}{r}0.0 \\
x x\end{array}$ & $\begin{array}{r}0.4 \\
30.0\end{array}$ & $\begin{array}{r}2.0 \\
45.0\end{array}$ & $\mathrm{mg} / 1$ & 0 & $\begin{array}{l}\text { Biweekly } \\
\text { Monthly }\end{array}$ & $\begin{array}{l}8 \mathrm{hr} . \\
8 \mathrm{hr} .\end{array}$ \\
\hline $\begin{array}{l}\text { Percent removal, } \\
\text { Suspended solids }\end{array}$ & $\begin{array}{l}\text { Sample measurement } \\
\text { Permit requirement }\end{array}$ & $\begin{array}{l}\mathrm{xx} \\
\mathrm{xx}\end{array}$ & $\begin{array}{l}\mathrm{xX} \\
\mathrm{xx}\end{array}$ & $\begin{array}{l}\mathrm{xx} \\
\mathrm{xx}\end{array}$ & - & - & $\begin{array}{l}95.0 \\
85.0\end{array}$ & $\begin{array}{r}98.8 \\
\mathrm{xX}\end{array}$ & $\begin{array}{r}100.0 \\
x x\end{array}$ & $\%$ & 0 & $\begin{array}{l}\text { Weeklyy } \\
\text { Monthly }\end{array}$ & $\begin{array}{l}- \\
-\end{array}$ \\
\hline $\begin{array}{l}\text { Settleable solids, } \\
\text { Influent }\end{array}$ & $\begin{array}{l}\text { Sample measurement } \\
\text { Permit requirement }\end{array}$ & $\begin{array}{l}\mathrm{xX} \\
\mathrm{xx}\end{array}$ & $\begin{array}{l}\mathrm{xX} \\
\mathrm{xx}\end{array}$ & $\begin{array}{l}\mathrm{xx} \\
\mathrm{xx}\end{array}$ & - & - & $\begin{array}{r}0.3 \\
\mathrm{xx}\end{array}$ & $\begin{array}{r}1.8 \\
\mathrm{xx}\end{array}$ & $\begin{array}{r}5.0 \\
\mathrm{XX}\end{array}$ & - & - & $\begin{array}{l}5 / 7 \\
\text { Daily }\end{array}$ & $\begin{array}{l}\text { Grab } \\
\text { Grab }\end{array}$ \\
\hline $\begin{array}{l}\text { Settleable solids, } \\
\text { Effluent }\end{array}$ & $\begin{array}{l}\text { Sample measurement } \\
\text { Permit requirement }\end{array}$ & $\begin{array}{l}x x \\
x x\end{array}$ & $\begin{array}{l}\mathrm{xX} \\
\mathrm{xx}\end{array}$ & $\begin{array}{l}\mathrm{xx} \\
\mathrm{xx}\end{array}$ & - & - & $\begin{array}{r}0.0 \\
x x\end{array}$ & $\begin{array}{r}0.0 \\
\mathrm{xx}\end{array}$ & $\begin{array}{r}0.0 \\
\mathrm{xx}\end{array}$ & - & - & $\begin{array}{l}5 / 7 \\
\text { Da1ly }\end{array}$ & $\begin{array}{l}\text { Grab } \\
\text { Grab }\end{array}$ \\
\hline $\begin{array}{l}\text { Residual Chlarine, } \\
\text { Effluent }\end{array}$ & $\begin{array}{l}\text { Sample measurement } \\
\text { Permit requirement }\end{array}$ & $\begin{array}{l}x x \\
x x\end{array}$ & $\begin{array}{l}\mathrm{xX} \\
\mathrm{xx}\end{array}$ & $\begin{array}{l}\mathrm{Xx} \\
\mathrm{xx}\end{array}$ & - & - & $\begin{array}{r}0.5 \\
x x\end{array}$ & $\begin{array}{r}1.0 \\
x x\end{array}$ & $\begin{array}{r}1.7 \\
\mathrm{xx}\end{array}$ & - & - & $\begin{array}{l}5 / 7 \\
\text { Daily }\end{array}$ & $\begin{array}{l}\text { Grab } \\
\text { Grab }\end{array}$ \\
\hline $\begin{array}{l}\text { Temperature, } \\
\text { Effluent }\end{array}$ & $\begin{array}{l}\text { Sample measurement } \\
\text { Permit requirement }\end{array}$ & $\begin{array}{r}6.0 \\
x x\end{array}$ & $\begin{array}{r}15.1 \\
\mathrm{xX}\end{array}$ & ${ }^{23} \mathrm{xx}$. & ${ }^{\circ} \mathrm{C}$ & - & $\begin{array}{l}\mathrm{xx} \\
\mathrm{xx}\end{array}$ & $\begin{array}{l}\mathrm{xx} \\
\mathrm{xx}\end{array}$ & $\begin{array}{l}\mathrm{XX} \\
\mathrm{xx}\end{array}$ & - & - & $\begin{array}{l}5 / 7 \\
\text { Daily }\end{array}$ & $\begin{array}{l}\text { Gráb } \\
\text { Grab }\end{array}$ \\
\hline $\begin{array}{l}\text { Fecal coliform, } \\
\text { Effluent }\end{array}$ & $\begin{array}{l}\text { Sample measurement } \\
\text { Permit requinement }\end{array}$ & & $\begin{array}{l}\mathrm{xx} \\
\mathrm{xx}\end{array}$ & $\begin{array}{l}x x \\
x x\end{array}$ & - & - & $\begin{array}{r}0.0 \\
\mathrm{XX}\end{array}$ & $\begin{array}{r}0.0 \\
200.0\end{array}$ & $\begin{array}{r}0.0 \\
400.0\end{array}$ & $\mathrm{n} / 100 \mathrm{ml}$ & 10 & $\begin{array}{l}\text { Weekly } \\
\text { Month1y }\end{array}$ & $\begin{array}{l}\text { Grab } \\
\text { Grab }\end{array}$ \\
\hline
\end{tabular}

$\mathrm{XX}$ Indicates not required.

a Total fo: the year.

Data collected by R. Richards and G. Yengel (Sewage Treatment Plant) and forwarded by H. Haller (Plant Engineering) to Safety and Envircnmental Protection Division. 


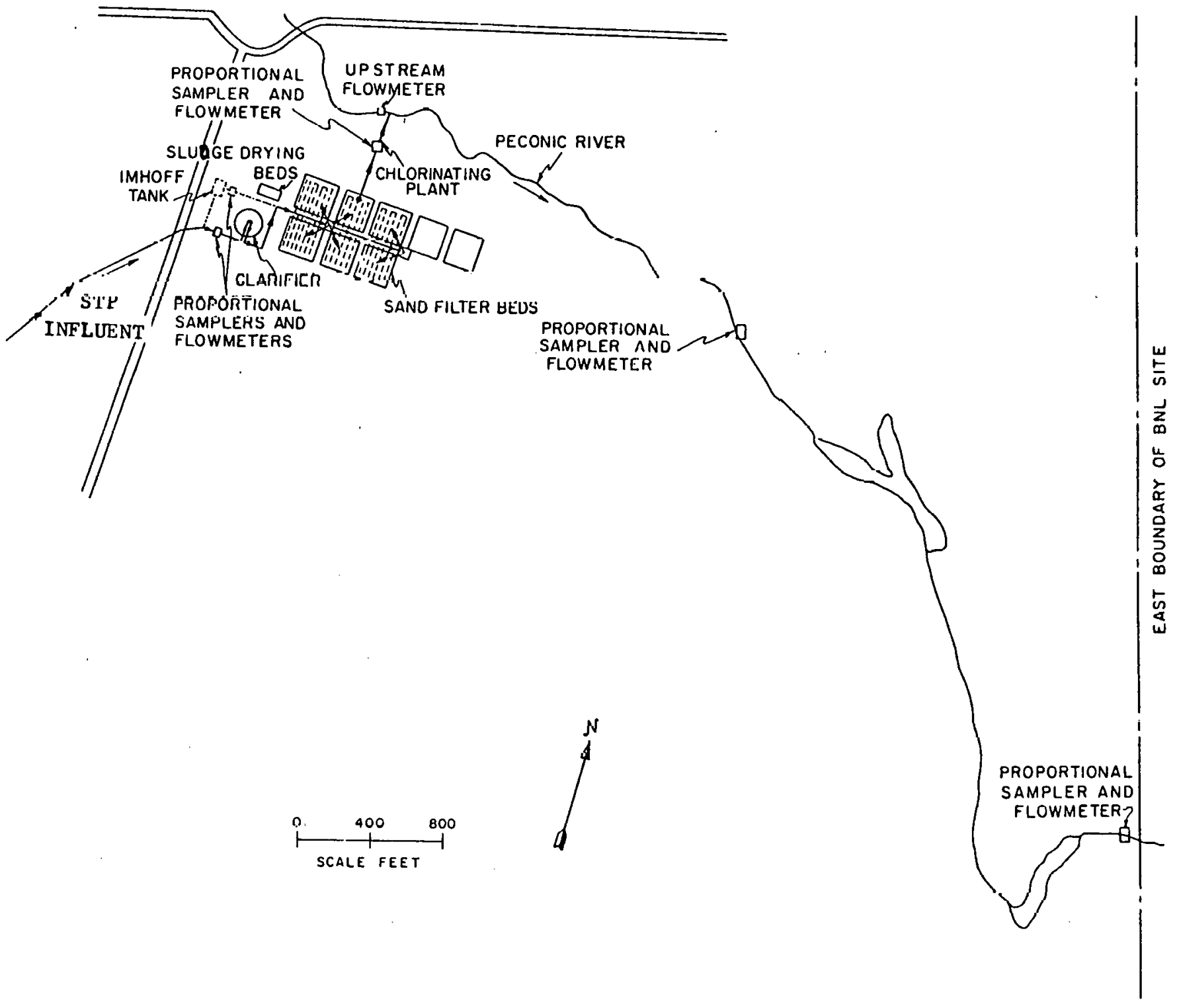

Figure 6. Sewage Treatment Plant 
cated at the chlorine house, at the former site boundary which is $0.8 \mathrm{~km}$ downstream on the Peconic River, and at the site boundary, $2.6 \mathrm{~km}$ downstream.

An aliquot of each daily (or weekend) sample of the input to the sand filter beds and of their output to the chlorine house outfall was evaporated for gross alpha and gross beta analysis, and another was counted directly for tritium analysis. Samples from the two downstream locations were obtained three times a week. Aliquots of each sample were analyzed for gross beta, alpha, and for tritium. Another aliquot, proportional to the measured flow during the sampling period, was passed through ion exchange colums for subsequent analysis as an integrated sample. If the gross beta count at each location did not indicate the need for immediate radionuclide identification, then one set of these columns was analyzed directly on a monthly or quarterly bas.is for gamma emitting nuclides and the other was eluted for radiochemical processing for ${ }^{90} \mathrm{Sr}$ analysis. The monthly average flow and the monthly totals of gross beta and principal nuclide activities at the clarifier (input to the filter beds) and at the chlorine house (output from the beds) are shown in Table 11 . Yearly totals and average concentrations are indicated. The average monthly flow at the clarifier which had decreased from previous years, during 1976 and 1977, increased in 1978 and 1979 an increase over the 1977 flows by $14 \%$ and $22 \%$ respectively. The output at the chlorine house has shown a similar increase. The loss to ground water through the sand filter beds, however, has apparently increased when compared to 1978, and could be the result of a combination of events, such as, calibration of flowmeters, loss of efficiency of filter beds, etc. This is being investigated. About $78 \%$ of the total flow into the clarifier appeared in the output at the chlorine house after passing through the sand filter beds. The balance was assumed to have percolated to the ground water flow under the beds. Estimates of the amount of radioactivity released to the ground water in this manner during 1979 are shown in Table 11. These were calculated on the additional as sumption that the average concentrations of the contained nuclides corresponded to those in the output from the beds, as observed at the chlorine house.

An analysis of the radionuclide concentrations at the chlorine house over the past six years (1974-1979) has indicated that the fluctuations observed appear to be relatable to sand filter beds. It also indicates that a time lag between input and output from from the sand filter beds is evident. This 1 ag appears to be greater for ${ }^{134} \mathrm{Cs}$ and $137 \mathrm{Cs}$ than for ${ }^{90} \mathrm{Sr}$. During 1979 , radionuclides such as $51 \mathrm{Cr}, 65 \mathrm{Zn}, 95 \mathrm{Zr}-\mathrm{Nb}, 125 \mathrm{Sb}, 131 \mathrm{I}$ and $144 \mathrm{Ce}$, which have been detected in previous years, were all at or below MDL (see Appendix B) and as such were not reported in the Table 11. The Laboratory releases of radionuclides have been on the decrease over the years as a result of the ALARA approach .

Flow and activity concentration information at the former site boundary sampling location, $0.8 \mathrm{~km}$ downstream (see Fig. 6), and at the present site boundary are shown in Table 12. The climatic conditions, which in 1977 had resulted in decreased flows when compared to previous years, were reversed in 1978 and 1979. Above average rainfall $(143 \mathrm{~cm}$ ) has resulted in a flow at the site perimeter which was nine times greater than that recorded in 1977 . It must be noted, however, that the flows recorded in 1979 were estimated on the basis of chloride 
TABLE 11

1979 BNL Environmental Monitoring Total Activities and Concentrations of Identifiable Nuclides in Liquid Effluents

Sewage Treatment Plant

\begin{tabular}{|c|c|c|c|c|c|c|c|c|c|c|c|c|c|c|}
\hline & Flow & & & $\mathrm{GB}$ & & & & & & & & & & \\
\hline Month & $10^{10} \mathrm{ml}$ & $\mathrm{Ga}$ & $G B$ & $+Y$ only ${ }^{a}$ & ${ }^{3} \mathrm{H}$ & ${ }^{7} \mathrm{Be}$ & ${ }^{22} \mathrm{Na}$ & ${ }^{54} \mathrm{Mn}$ & ${ }^{57} \mathrm{Co}$ & ${ }^{58} \mathrm{Co}$ & ${ }^{60} \mathrm{Co}$ & ${ }^{90} \mathrm{Sr}$ & $134 \mathrm{Cs}$ & ${ }^{137} \mathrm{Cs}$ \\
\hline \multicolumn{15}{|c|}{ Clarifier (mCi) } \\
\hline January & 12.59 & 0.46 & 21.8 & 33.41 & 1305 & 1.9 & 0.41 & $\mathrm{~b}$ & $\mathrm{~b}$ & $\mathrm{~b}$ & $\mathrm{~b}$ & 0.038 & 3.40 & 5.7 \\
\hline February & 10.61 & 0.21 & 8.4 & 10.93 & 467 & 0.2 & 0.05 & .03 & $b$ & $\mathrm{~b}$ & b & 0.017 & 0.63 & 1.6 \\
\hline April & 13.22 & 0.20 & 6.1 & 6.37 & 1553 & 0.15 & 0.001 & .003 & .02 & .05 & $b$ & 0.033 & .05 & .08 \\
\hline May & 14.79 & 0.22 & 3.4 & 3.58 & 636 & 0.09 & b & .01 & .02 & $\mathrm{~b}$ & .04 & 0.050 & .02 & b. \\
\hline June & 14.42 & 0.21 & 2.8 & 2.94 & 1177 & .04 & b & .01 & .02 & b & .04 & 0.023 & .02 & .007 \\
\hline July & 16.66 & 0.22 & 4.3 & 4.47 & 2523 & .05 & b & .02 & .02 & $\mathrm{~b}$ & .05 & 0.017 & .02 & .008 \\
\hline August & 15.49 & 0.20 & 3.3 & 3.42 & 4114 & .06 & .01 & וח. & .01 & $\mathrm{~h}$ & ก? & $n$ กอ? & b & .01 \\
\hline September & 11.74 & 0.15 & 10.3 & 11.41 & 1769 & b & b & b & .06 & $\mathbf{b}$ & 1.05 & 0.024 & $\mathrm{~b}$ & $\mathrm{~b}$ \\
\hline October & 16.92 & 0.20 & 4.3 & 4.64 & 3823 & 0.16 & b, & $h$ & $\mathrm{~h}$ & $\mathrm{~h}$ & ก. 16 & 0.036 & .02 & $\mathrm{~b}$ \\
\hline November & 13.29 & $0.1 \overline{7}$ & 4.0 & 4.68 & 2551 & 0.20 & b & $\mathrm{h}$ & $\mathrm{h}$ & $h$ & $\cap 4 a$ & 0.017 & .04 & b \\
\hline December & 10.96 & 0.17 & 2.9 & $3.4 \overline{7}$ & 1135 & 0.19 & b & $\mathrm{b}$ & $b$ & 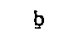 & 0.3 & 0.045 & ก.กא & ก.ก? \\
\hline \multirow[t]{4}{*}{ Total } & 162.81 & 2.58 & 75.2 & 94.16 & 21810 & 1.27 & 0.41 & .092 & 0.18 & .07 & 2.22 & 0.347 & 5.11 & 7.60 \\
\hline & \multicolumn{14}{|c|}{ 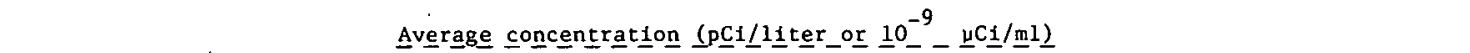 } \\
\hline & & 1.58 & 46.19 & 57.80 & 13400 & 0.78 & 0.25 & .06 & 0.10 & .04 & 1.36 & 0.21 & 3.14 & 4.67 \\
\hline & & & & & Groundw & ater ( & nd-Filt & er Beds & (mCl & & & & & \\
\hline \multirow[t]{3}{*}{ Total } & 36.17 & 0.51 & 10.3 & 12.61 & 4310 & 0.43 & 0.13 & .06 & 0.03 & $\mathrm{~b}$ & 0.09 & 0.19 & 0.57 & 1.00 \\
\hline & \multicolumn{14}{|c|}{ 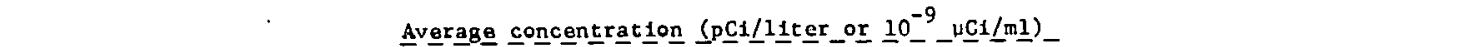 } \\
\hline & & 1.4 & 28.5 & 34.86 & 11910 & 1.20 & 0.37 & 0.17 & 0.09 & $\mathrm{~b}$ & 0.26 & 0.53 & 1.58 & 2.77 \\
\hline
\end{tabular}

\begin{tabular}{|c|c|c|c|c|c|c|c|c|c|c|c|c|c|c|}
\hline \multirow[b]{2}{*}{ January } & \multicolumn{14}{|c|}{ Chlorine House ( $\mathrm{mC} 1$ ) } \\
\hline & 10.41 & 0.19 & 3.6 & 4.94 & 887 & 0.75 & 0.27 & U. U's & b & b & .005 & 0.072 & 0.21 & 0.28 \\
\hline February & 7.74 & 0.12 & 1.8 & 2.93 & 256 & 0,40 & 0.09 & $\mathrm{~h}$ & n.ก2. & $\mathrm{h}$ & $n .03$ & ก.ก7n & 0.23 & 0.36 \\
\hline March & 10.12 & 0.16 & 2.1 & 2.81 & 624 & 0.14 & 0.02 & 0.05 & 0.01 & $\mathrm{~b}$ & 0.04 & 0.073 & 0.16 & 0.34 \\
\hline April & 10.46 & 0.16 & 3.8 & 4.19 & 1669 & b & 0.01 & 0.04 & 0.02 & $\mathrm{~b}$ & 0.06 & 0.061 & 0.18 & 0.24 \\
\hline May & 11.80 & 0.18 & 3.0 & 3.13 & 506 & b & 0.001 & 0.02 & b & $\mathrm{b}$ & 0.02 & 0.063 & 0.08 & 0.12 \\
\hline June & 14.78 & 0.22 & 3.5 & 3.62 & 860 & $h$ & $\mathrm{~h}$ & $n \cdot \cap ?$ & $n . n ?$ & b & 0.05 & 0.096 & 0.03 & 0.20 \\
\hline July & 12.46 & n.16 & 2.8 & 3.51 & 1957 & b & & 0.03 & b & $b$ & L & 0.062 & 0.23 & 0.43 \\
\hline Nuguat & 11.57 & 0.14 & $2 . \hat{2}$ & 3.11 & 2903 & 0.16 & 0.03 & $\dot{U} \cdot \dot{U} 2$ & $\mathbf{b}$ & b & b & U.011 & 0.2 .3 & 0.47 \\
\hline September & 8.22 & 0.09 & 6.5 & 7.66 & 823 & $b$ & h & $\mathrm{h}$ & $h$ & $\mathrm{~h}$ & n.mก? & n. n29 & ก 16 & 0.27 \\
\hline OcLuber & 10.20 & 0.12 & 3.1 & 3.11 & 2382 & 0.10 & 0.02 & 0.01 & 0.01 & $b$ & 0.01 & 0.048 & 0.15 & 0.29 \\
\hline November & 12.25 & 0.16 & 2.5 & 2.99 & 1597 & $b$ & 0.01 & 0.01 & 0.01 & b & 0.05 & 0.047 & 0.14 & 0.27 \\
\hline December & 6.97 & 0.08 & 1.3 & 1.67 & 653 & $b$ & 0.01 & 0.01 & b & b & 0.04 & 0.024 & 0.11 & 0.20 \\
\hline Total & 127.0 & 1.78 & 36.2 & 44.27 & 15120 & 1.52 & 0.47 & 0.22 & 0.12 & b & 0.33 & 0.676 & 2.01 & 3.52 \\
\hline
\end{tabular}

A

\begin{tabular}{|c|c|c|c|c|c|c|c|c|c|c|c|c|c|}
\hline [18] and Radiation & $\begin{array}{l}\text { regulations } 1.4 \\
\text { Concentration }\end{array}$ & 28.5 & $\begin{array}{l}34.86 \\
n^{3} \mathrm{c}\end{array}$ & $\begin{array}{l}11910 \\
2 \times 10^{4}\end{array}$ & $\begin{array}{l}1.20 \\
2 \times 10^{6}\end{array}$ & $\begin{array}{l}0.37 \\
4 \times 10^{4}\end{array}$ & $\begin{array}{l}0.17 \\
1 \times 10^{5}\end{array}$ & $\begin{array}{l}0.09 \\
5 \times 10^{4}\end{array}$ & $1 \times 10^{5}$ & $\begin{array}{r}0.26 \\
5 \times 10^{4}\end{array}$ & $0.5 ?$ & $\begin{array}{r}1.58 \\
9 \times 10^{3}\end{array}$ & $\begin{array}{l}2.77 \\
2 \times 10^{4}\end{array}$ \\
\hline
\end{tabular}

ancludes gamma (only) emitters but excludes tritium.

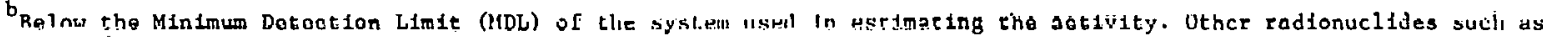
${ }^{51} \mathrm{Cr},{ }^{65} \mathrm{Zn},{ }^{95} \mathrm{ZrNb},{ }^{125} \mathrm{Sb},{ }^{131} \mathrm{I},{ }^{140} \mathrm{BaLa},{ }^{144} \mathrm{Ce}$ were al1 below MDL

$c_{\text {For mixtures of radionuclides containing }<10 \%}{ }^{90} \mathrm{Sr}, 125-133 \mathrm{I}$, or long lived alpha emitters. The concentration guides for unknown mixtures depend, within the range given, on whether certaln radionuclides are know to be present in concentrations less than 0.1 of their CGs, and the sum of the fractions of the CGs for all such nuclides is less than 0.25 .

$\mathrm{mC1}=3.7 \times 10^{7} \mathrm{~Bq}$.

$\mu \mathrm{Cl}=3.7 \times 10^{4} \mathrm{~Bq}$.

$\mathrm{pC} 1=3.7 \times 10^{-2} \mathrm{~Bq}$. 
TABLE 12

1979 BNL Environmental Monitoring Total Activities and Concentrations of Identifiable

Nuclides in Liquid Effluents, Peconic River

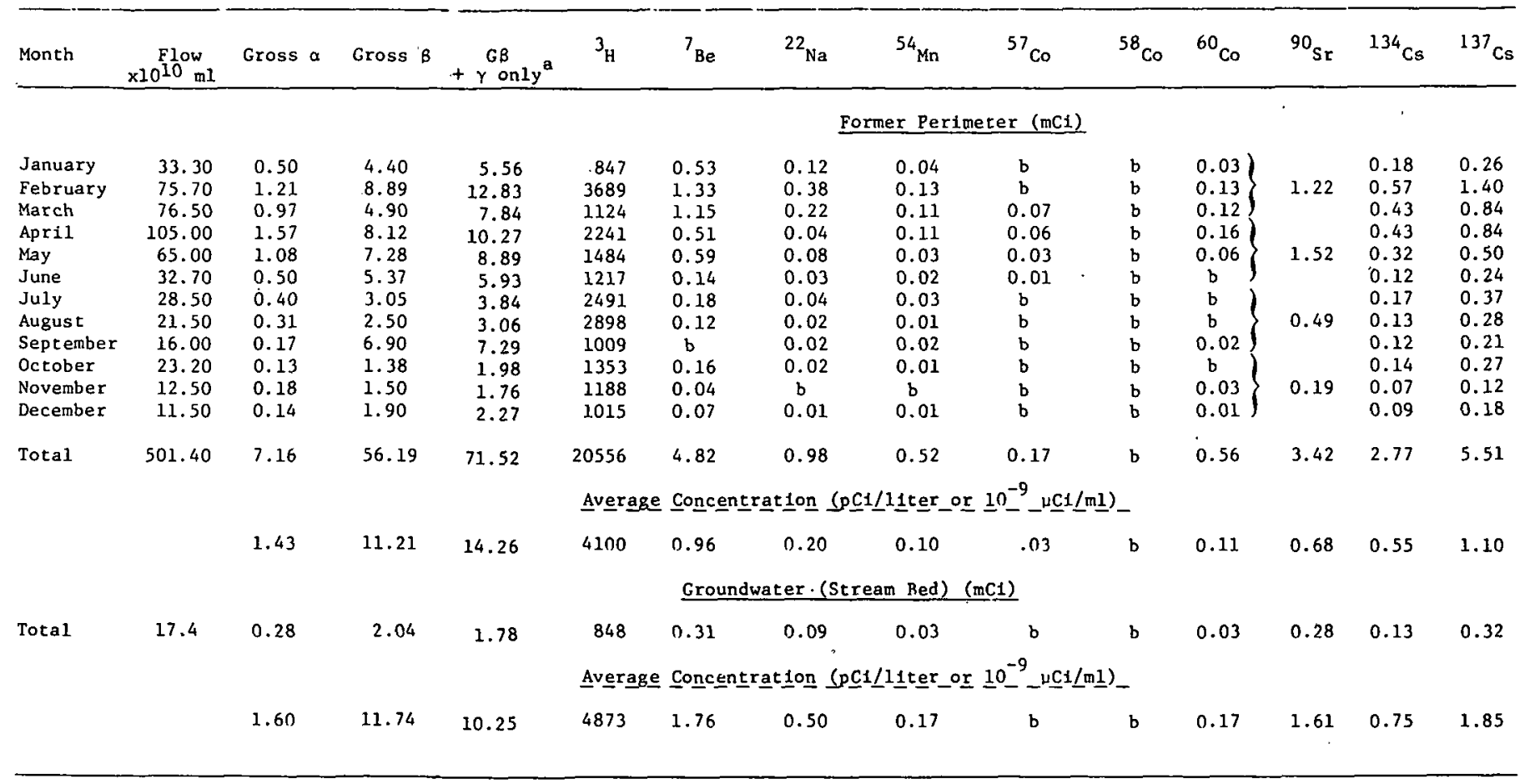

\begin{tabular}{|c|c|c|c|c|c|c|c|c|c|c|c|c|c|c|}
\hline January & 36.40 & 0.54 & 4.40 & 4.88 & 847 & 0.23 & 0.03 & 0.01 & 0.01 & b & $0.01)$ & \multirow[b]{2}{*}{1.43} & 0.08 & 0.11 \\
\hline February & 58.30 & 0.87 & 5.60 & 8.19 & 960 & 1.69 & 0.26 & 0.05 & 0.03 & $b$ & $0.01\}$ & & 0.11 & 0.44 \\
\hline March & 77.80 & 1.00 & 5.00 & 9.96 & 10090 & 1.01 & 0.36 & 0.09 & b & b & 0.18 & & 0.28 & 0.63 \\
\hline April & 112.00 & 1.62 & 21.20 & 25.03 & 3479 & 0.43 & b & 0.21 & $\mathrm{~b}$ & b & 0.10 & \multirow{3}{*}{0.44} & 0.03 & 0.10 \\
\hline May & 68.90 & 1.04 & 7.38 & 8.43 & 2022 & 0.39 & 0.05 & 0.05 & 0.05 & b & b & & 0.02 & 0.49 \\
\hline June & 34.10 & 0.46 & 7.70 & 8.0 & 648 & 0.11 & 0.01 & b & $\mathrm{b}$ & b & $b$ & & 0.06 & 0.12 \\
\hline July & 29.20 & 0.40 & 16.60 & 17.17 & 1216 & 0.11 & 0.02 & 0.03 & 0.01 & b & 0.02 & \multirow{3}{*}{0.27} & 0.12 & 0.26 \\
\hline August & 23.30 & 0.31 & 2.70 & 3.12 & 2368 & 0.09 & 0.01 & 0.01 & $\mathrm{~b}$ & b & b & & 0.10 & 0.21 \\
\hline September & 23.30 & 0.26 & 3. 30 & 3.74 & 1067 & $b$ & 0.02 & 0.02 & 0.05 & $b$ & 0.02 & & 0.12 & 0.21 \\
\hline October & 25.10 & 0.30 & 2.33 & 2.83 & 2774 & 0.14 & 0.02 & 0.01 & $b$ & $\mathrm{~b}$ & $\mathrm{~b}$ & \multirow{3}{*}{0.33} & 0.10 & 0.23 \\
\hline November & 16.20 & 0.21 & 1.70 & 1.95 & 1834 & 0.06 & b & $\mathrm{b}$ & $\mathrm{b}$ & b & $\mathbf{b}$ & & 0.07 & 0.12 \\
\hline December & 14.99 & 0.18 & 1.80 & 1.84 & 739 & $\mathrm{~b}$ & b & b & $\mathrm{b}$ & $b$ & $\mathrm{~b}$ & & 0.01 & 0.03 \\
\hline \multirow[t]{3}{*}{ Total } & 519.49 & 7.19 & 79.71 & 95.14 & 28044 & 4.26 & 0.78 & 0.48 & 0.15 & b & 0.34 & 2.47 & 1.10 & 2.95 \\
\hline & & \multicolumn{13}{|c|}{ 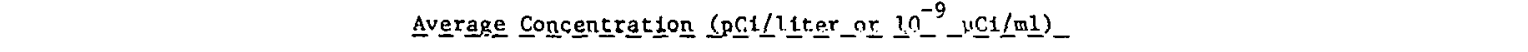 } \\
\hline & & 1.38 & 15.35 & 18.31 & 5399 & 0.82 & 0.15 & 0.10 & 0.03 & b & 0.07 & 0.48 & 0.21 & 0.57 \\
\hline \multicolumn{2}{|c|}{$\begin{array}{l}\text { EPA-Drinking Water } \\
\text { Regulations }[18] \text { and } \\
\text { Radiation Conc Guides }\end{array}$} & {$[17]$} & \multicolumn{2}{|c|}{$3 \times 10^{3 c}$} & $2 \times 10^{4}$ & $2 \times 10^{6}$ & $4 \times 10^{4}$ & $1 \times 10^{5}$ & $5 \times 10^{5}$ & $1 \times 10^{5}$ & $5 \times 10^{4}$ & 8 & $9 \times 10^{3}$ & $2 \times 10^{4}$ \\
\hline
\end{tabular}

ancludes gamma (only) emitters but excludes tritium.

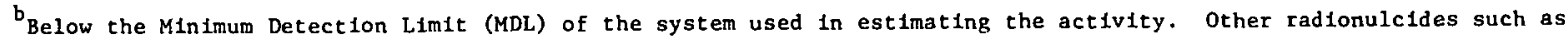

${ }^{51} \mathrm{Cr},{ }^{65} \mathrm{Zn},{ }^{95} \mathrm{ZrNb},{ }^{125} \mathrm{Sb},{ }^{131} \mathrm{I},{ }^{140} \mathrm{Ce}$ were al1 below MDL.

Fur mixlures of radionuclides containing $410 \%{ }^{90} 5 \mathrm{r}, 125-133 \mathrm{I}$, or long 11ved alpha emitters. The concentration guides for unknown mixtures depend, within the range given, on whether certain radionulcides are known to be present in concentrations less than $0: 1$ of the1r CGs, and the sum of the fractlons of the CGs for all such nuclides is less than 0.25 .

$\mathrm{mCl}=3.7$ c $10^{7} \mathrm{~Bq}$.

$\mu \mathrm{Cl}=3.7 \times 10^{4} \mathrm{~Bq}$.

$\mathrm{pCi}=3.7 \times 10^{-2} \mathrm{~Bq}$. 
concentrations, since the flows exceeded the capability of the measuring system. A review of the flow measuring system is in progress. Such changes in flow do affect the amount of water that percolates into the ground water system; for example, $16 \%$ of the flow between the former perimeter and the present site boundary was lost to the ground water in $1975,30 \%$ in $1976,60 \%$ in 1977 less than $1 \%$ in 1978 and about $3 \%$ in 1979. Such fluctuations can result in changes in radionuclide activity concentrations between the former and present site boundary but this is related to total flow since the total activity essentially remains almost constant. This is true for radionuclides, such as $7_{\mathrm{Be},} 22 \mathrm{Na}$, and $137 \mathrm{Cs}$, which have remained in solution and therefore have not significantly precipitated out of the water body. Upper limit estimates of the total activity that may have percolated to the underlying aquifier is also shown in Table 12 . These are based on the decrease in total flow between the former site boundary and the perimeter during October and November.

Analysis of monthly composite samples of the Peconic River at the former site boundary $(0.8 \mathrm{~km}$ downstream from the chlorine house) during this period showed that, on the average, $4 \%$ of the annual total activity (excluding tritium) consisted of $90 \mathrm{Sr}$ and that no appreciable amounts of long-lived radioactive iodine or other bone-seeking nuclides such as radium were prèsent. It must be noted that the $4 \%$ indicated above would decrease if other radionuclides were present. Under these circumstances, the applicable RCG was $3000 \mathrm{pCi} 1^{-1}(3.0 \mathrm{x}$ $10^{-6} \mu \mathrm{Ci} \mathrm{ml}-1$ or $\left.1.1 \times 10^{-1} \mathrm{~Bq} \mathrm{ml} 1^{-1}\right)$. The gross beta concentration in the portion which is assumed to have percolated to ground water was $11.7 \mathrm{pCi} 1^{-1}$ (1.17 $x 10^{-8} \mu \mathrm{Ci} \mathrm{ml}^{-1}$ or $4.32 \times 10^{-4} \mathrm{~Bq} \mathrm{ml}{ }^{-1}$ ) or $<1 \%$ of the RCG.

At the Laboratory perimeter ( $2.6 \mathrm{~km}$ downstream from the chlorine house), $3 \%$ of the annual total activity was $90 \mathrm{Sr}$. The applicable RCG was also $3000 \mathrm{pCi}$ $1^{-1}\left(1.1 \times 10^{-1} \mathrm{~Bq} \mathrm{~m} 1^{-1}\right)$. The observed gross beta concentration of the water released downstream was $15.4 \mathrm{pCi} 1^{-1}\left(1.54 \times 10^{-8} \mu \mathrm{Ci} \mathrm{ml}^{-1}\right.$ or $\left.5.7 \times 10^{-4} \mathrm{~Bq} \mathrm{ml}^{-1}\right)$ or $<1 \%$ of the RCG.

In addition to the above measurements, the Safety and Environmental Protection Division conducts routine measurements of water quality and purity of the filter beds effluent, upstream of the Peconic River, at the former perimeter of the Laboratory $(0.8 \mathrm{~km}$ downstream) and at the present Laboratory perimeter (2.6 km downstream). A summary of these data for 1979 is shown in Table 13. The outflow from the sand filter beds (EA) into the Peconic River complied with water quality standards for minimum dissolved oxygen (DO) (12,19), except at upstream of the outfal1 ( $\mathrm{HE}$ ) and at the perimter (HM). During the severe winter of $1977 / 1978$ there was heavy snowfall. This and the subsequent rise in water table in 1979 resulted in heavy decomposition of vegetation due to water logging in the Peconic River drainage area. This would lead to a high content of humic acid in the river water, which in turn would increase the biological oxygen demand and thereby lead to reductions in dissolved oxygen content, as indicated (20). Although occasionally below the standard (19), the effluent $\mathrm{pH}$ was within the range of local ambient levels. After mixing with the upstream flow, the temperature increment was within the standard (21) at the Laboratory perimeter. Yearly average concentrations of most metals were, before dilution, at or within the standard for the receiving body of water $(12,19)$ except iron $(\mathrm{Fe})$ and occasionally copper $(\mathrm{Cu})$, and lead $(\mathrm{Pb})$. From past studies, iron ( $\mathrm{Fe}$ ) 
TABLE 13

1979 BNL ENVIRONMENTAL MONI TORING LIOUID EFFLUENT WATER OUALITY AND PURITY

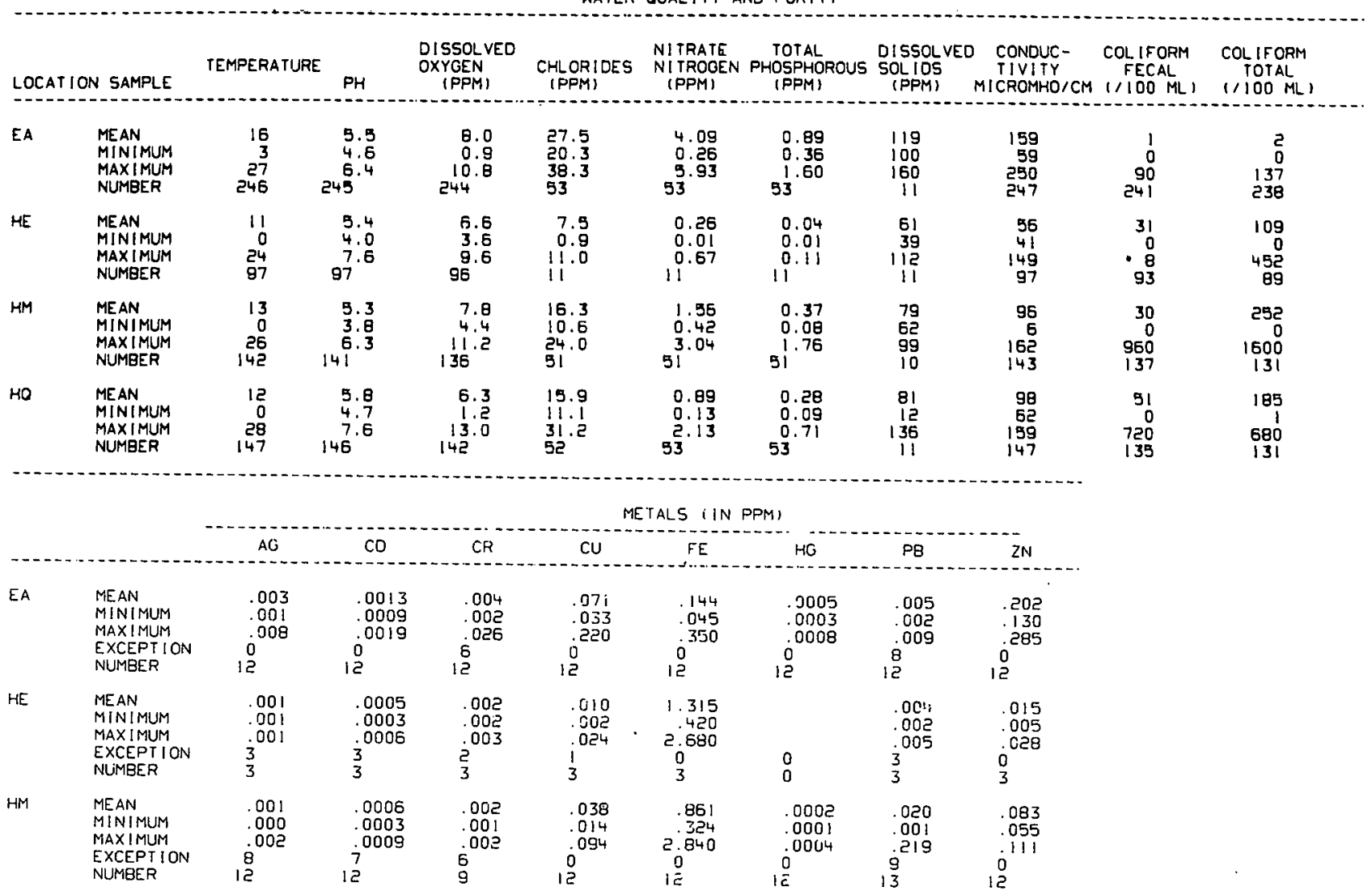

1979 BNL ENVIRONMENTAL MONITORING SEWAGE INFLUENT WATER QUAL ITY AND PURITY METALS

\begin{tabular}{|c|c|c|c|c|c|c|c|c|}
\hline & & $A G$ & $\mathrm{CD}$ & $\mathrm{CR}$ & TALS II & $\mathrm{FE}$ & $\mathrm{PB}$ & $\mathrm{ZN}$ \\
\hline DA & $\begin{array}{l}\text { MEAN } \\
\text { MINIMUM } \\
\text { MAXIMUM } \\
\text { EXCEP!IUN } \\
\text { NUMBER }\end{array}$ & $\begin{array}{l}.004 \\
.001 \\
.007 \\
12\end{array}$ & $\begin{array}{l}.0015 \\
.0008 \\
.0025 \\
0^{12}\end{array}$ & $\begin{array}{l}.008 \\
.002 \\
.019 \\
\dot{2}^{12}\end{array}$ & $\begin{array}{l}.078 \\
.033 \\
.181 \\
0 \\
12\end{array}$ & $\begin{array}{l}.331 \\
.213 \\
.510 \\
0 \\
12\end{array}$ & $\begin{array}{l}.007 \\
.003 \\
i^{011} \\
1 \mathrm{i}^{-011}\end{array}$ & $\begin{array}{l}.112 \\
.078 \\
.197 \\
0 \\
. \check{~}\end{array}$ \\
\hline
\end{tabular}

1979 BNL ENVIRONMENTAL MONITORING PRECIPITATION WATER QUALITY AND PURITY

\begin{tabular}{|c|c|c|c|c|c|c|c|c|c|c|c|}
\hline LOCATION & N SAMPLE & TEMPERATURE & $\mathrm{PH}$ & $\begin{array}{l}\text { DISSOL VF.D } \\
\text { OXYGEN } \\
\text { (PPM) }\end{array}$ & $\begin{array}{l}\text { CHLORIOES } \\
\text { (PPM) }\end{array}$ & $\begin{array}{l}\text { NiTRATE } \\
\text { Ni TROGEN } \\
\text { (PPM) }\end{array}$ & $\begin{array}{l}\text { TOTAL } \\
\text { PHOSPHOROUS } \\
\text { (PPM) }\end{array}$ & $\begin{array}{l}\text { OISSOLVED } \\
\text { SOL IOS } \\
\text { (PPM) }\end{array}$ & $\begin{array}{l}\text { CONDUC- } \\
\text { TIVITY } \\
\text { MICROMHO/CM }\end{array}$ & $\begin{array}{l}\text { COL IFORM } \\
\text { FECAL } \\
(/ 100 \mathrm{ML})\end{array}$ & $\begin{array}{c}\text { COL IFORM } \\
\text { TOTAL } \\
1 / 100 \mathrm{ML})\end{array}$ \\
\hline GD & $\begin{array}{l}\text { MEAN } \\
\text { MINIMUM } \\
\text { MAXIMUM } \\
\text { NUMBER }\end{array}$ & 0 & $\begin{array}{r}4.1 \\
3.0 \\
5.8 \\
52\end{array}$ & 0 & 0 & 0 & 0 & 0 & 0 & 0 & 0 \\
\hline
\end{tabular}

Reference Standards - Table 29

FA: Sand filter beds

HE: Upstreali of uulfall

HM: Former site boundary
Fxception: No. of samples below

Minimum Detection Limit (MDL)

HQ: SIte houndary

DA: Sewage Effluent

GD: Precipitation 
seems to be ubiquitous at the levels seen in the ground water system. Since the Laboratory derives all of its water from the underlying aquifer, the presence of Fe in our effluents is not surprising. The high value of Cu upstream of the outfall is consistent with the presence of humic acid, which is known to chelate and concentrate transition metals from the water (20).

Monthly "grab" water samples were obtained at on- and off-site locations along the upper tributary of the Peconic River, into which the Laboratory routinely discharges low level radioactive liquids within administrative limits (15). A battery operated fixed flow sampler is operated at Riverhead, at the mouth of the Peconic River, between March and December. Reference "grab" samples were obtained from other nearby streams and bodies of water outside the Laboratory drainage area. The sampling locations, as shown in Figure 7 , were as follows:

Uff-Site (Peconic Kiver, proreeding dnwstream)

A - Peconic River at Schultz Road, $4.85 \mathrm{~km}$ downstream (HA),

B - Peconic River at Wading River-Manorville Road, $7.04 \mathrm{~km}$ downstream (HB),

C - Peconic River at Manorville, $10.67 \mathrm{~km}$ downstream (HC),

D - Peconic River at Calverton, $14.23 \mathrm{~km}$ downstream (HD),

$\mathrm{R}$ - Peconic River at Riverhead, $19.35 \mathrm{~km}$ downstream (HR),

Controls (Not in the Laboratory drainage area)

E - Peconic River, upstream from the Laboratory effluent outfall (HE),

F - Peconic River, mith Libulary (independenl of the Laboratory (HF) drainage area),

H - Carman River, outfall of Yaphank Lake (HH),

I - Northeast corner of Artist Lake on Route 25 (HI).

Individual month $1 \mathrm{y}$ and yearly average gross beta, tritium and ${ }^{90}$ Sr concentrations at downstream and control locations are shown in Table 14 . A comparison with the on-site and perimeter concentrations shown in Table 12 indicates that the concentrations of Laboratory effluents in the Peconic River, downstream of the outfall, diminish rapidly to near background levels at the more distant sampling locations. Considering the concentrations of radioactivity near the mouth of the Peconic River at Riverhead, where the flow over the years has been about 19 times that at the Laboratory perimeter (USGS-1979 data), it was evident that the total amount of radioactivity at this location was much greater than those released into the Peconic River at the Laboratory perimeter. This obviously represents radioactivity washed out of drainage areas and tributary additions, in addition to that from the Laboratory area, by rainfall. During 


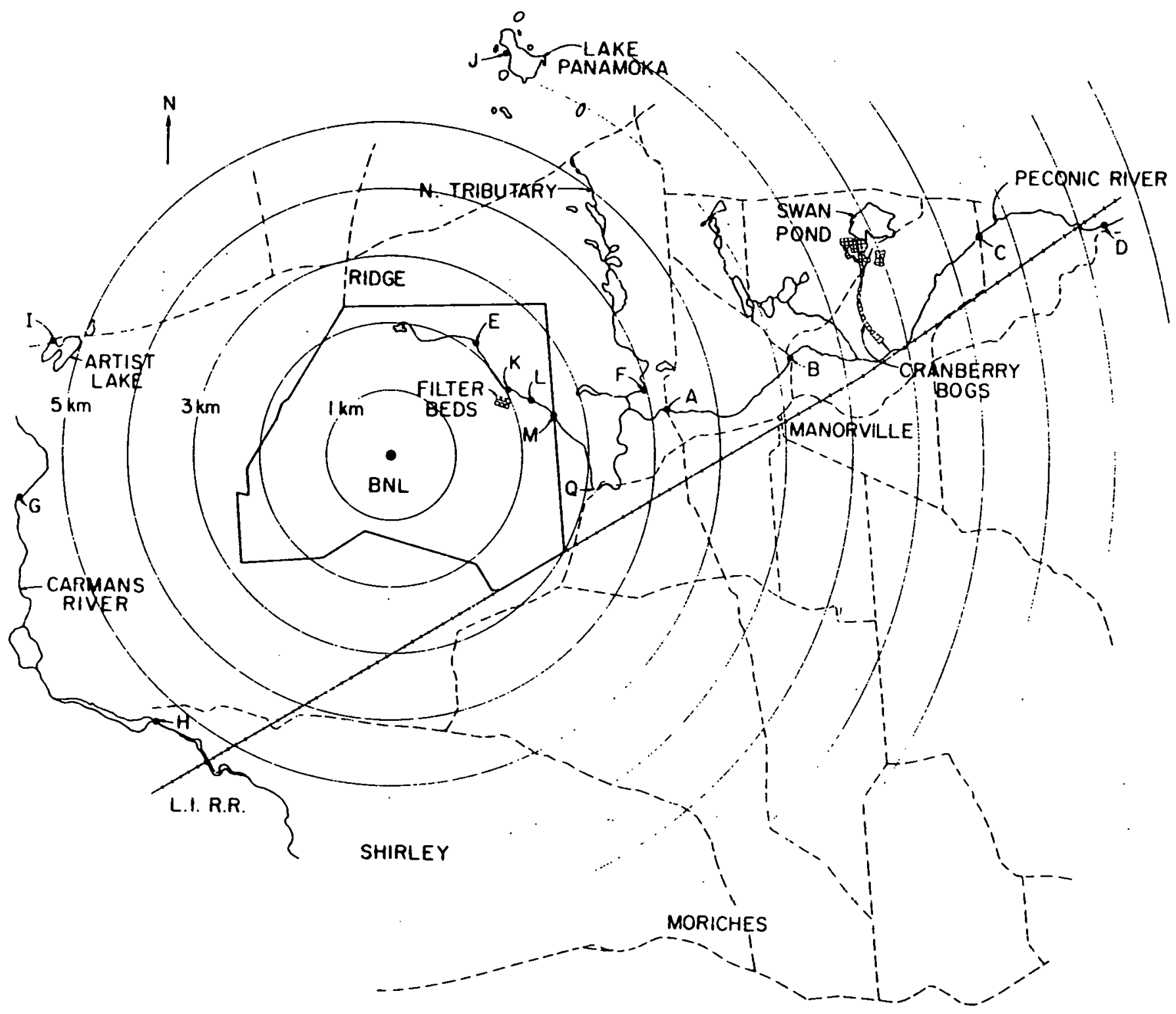

Figure 7. Peconic River, On-site and Downstream Sampling Locations 
TARLE 14

1979 BNL DUWNSTREAM AND CONTROL WATER SAMPLES

\begin{tabular}{|c|c|c|c|c|c|c|c|c|c|c|}
\hline LOCATION & QUARTER & $\begin{array}{l}\text { NO. OF } \\
\text { SAMPLES }\end{array}$ & $\begin{array}{c}\text { GROSS } \\
\text { ALPHA } \\
\text { (PCI I ) }\end{array}$ & ERROR & $\begin{array}{l}\text { GROSS } \\
\text { BETA } \\
\text { IPCI IL! }\end{array}$ & ERROR & $\begin{array}{l}\text { TRITIUM } \\
\text { (NCIIL) }\end{array}$ & ERROR & $\begin{array}{l}\text { SRSO } \\
(P C I / L)\end{array}$ & ERROR \\
\hline \multicolumn{11}{|l|}{ DOWNSTREAM } \\
\hline HA & $\begin{array}{l}\text { F.IRST } \\
\text { SECOND } \\
\text { THIRD } \\
\text { FOURTH }\end{array}$ & $\begin{array}{l}1 \\
1 \\
1 \\
1\end{array}$ & $\begin{array}{l}0.33 \\
0.56 \\
0.31 \\
0.26\end{array}$ & $\begin{array}{l}0.26 \\
0.37 \\
0.23 \\
0.26\end{array}$ & $\begin{array}{r}2.99 \\
5.38 \\
16.28 \\
7.35\end{array}$ & $\begin{array}{l}0.87 \\
1.07 \\
1.43 \\
1.13\end{array}$ & $\begin{array}{l}0.22 \\
0.32 \\
0.86 \\
0.90\end{array}$ & $\begin{array}{l}0.22 \\
0.32 \\
0.25 \\
0.26\end{array}$ & 0.62 & $\begin{array}{l}0.13 \\
0.11\end{array}$ \\
\hline \multicolumn{2}{|c|}{ SUMMARY } & 4 & 0.37 & 0.14 & 8.00 & 0.57 & $0.5 \%$ & 0.13 & 0.75 & 0.09 \\
\hline$H B$ & $\begin{array}{l}\text { F IRST } \\
\text { SECOND } \\
\text { THIRD } \\
\text { FOURTH }\end{array}$ & $\begin{array}{l}1 \\
1 \\
1 \\
1\end{array}$ & $\begin{array}{l}0.26 \\
0.42 \\
0.22 \\
0.24\end{array}$ & $\begin{array}{l}0.26 \\
0.36 \\
0.22 \\
0.24\end{array}$ & $\begin{array}{l}5.01 \\
6.30 \\
4.96 \\
4.14\end{array}$ & $\begin{array}{l}1.21 \\
1.14 . \\
0.99 \\
1.01\end{array}$ & $\begin{array}{l}0.22 \\
0.32 \\
0.32 \\
0.62\end{array}$ & $\begin{array}{l}0.22 \\
0.32 \\
0.32 \\
0.26\end{array}$ & $\begin{array}{l}0.69 \\
0.53\end{array}$ & $\begin{array}{l}0.13 \\
0.12\end{array}$ \\
\hline \multicolumn{2}{|c|}{ SUMMARY } & 4 & 0.29 & 0.14 & 5.10 & 0.55 & 0.37 & 0.14 & 0.61 & 0.09 \\
\hline$H C$ & $\begin{array}{l}\text { FIRST } \\
\text { EECOND } \\
\text { THIRC } \\
\text { FOURTH }\end{array}$ & $\begin{array}{l}1 \\
1\end{array}$ & $\begin{array}{l}0.31 \\
0.37 \\
0.34 \\
0.10\end{array}$ & $\begin{array}{l}0.31 \\
0.28 \\
0.23 \\
0.18\end{array}$ & $\begin{array}{l}3.55 \\
6.05 \\
7.95 \\
3.31\end{array}$ & $\begin{array}{l}0.90 \\
1.07 \\
1.14 \\
0.95\end{array}$ & $\begin{array}{l}0.22 \\
0.32 \\
0.32 \\
1.18\end{array}$ & $\begin{array}{l}0.2 .2 \\
0.32 \\
0.303 \\
0.28\end{array}$ & $\begin{array}{l}0.87 \\
0.47\end{array}$ & $\begin{array}{l}0.14 \\
0.11\end{array}$ \\
\hline \multicolumn{2}{|c|}{ SUMMARY } & 4 & 0.27 & 0.13 & 5.21 & $0.5 !$ & 0.51 & 0.14 & 0.72 & 0.09 \\
\hline Hח & $\begin{array}{l}\text { FIRSI } \\
\text { SECOND } \\
\text { THIRO } \\
\text { FOURTII }\end{array}$ & $\begin{array}{l}1 \\
1 \\
1 \\
1\end{array}$ & $\begin{array}{l}11.24 \\
0.24 \\
0.25 \\
0.18\end{array}$ & $\begin{array}{l}11.34 \\
0.24 \\
0.25 \\
0.18\end{array}$ & $\begin{array}{l}3.14 \\
3.48 \\
3.89 \\
1.35\end{array}$ & $\begin{array}{l}11.54 . \\
0.93 \\
0.94 \\
1.20\end{array}$ & $\begin{array}{l}\text { y. } 2 \mathrm{c} \\
0.32 \\
0.32 \\
1.33\end{array}$ & $\begin{array}{l}\text { U. } 55 \\
0.32 \\
0.32 \\
0.31\end{array}$ & $\begin{array}{l}0.70 \\
0.46\end{array}$ & $\begin{array}{l}0.13 \\
0.10\end{array}$ \\
\hline \multicolumn{2}{|c|}{ SUMMARY } & 4 & 0.24 & 0.12 & 3.88 & 0.50 & 0.55 & 0.15 & 0.58 & 0.08 \\
\hline $\mathrm{HL}$ & $\begin{array}{l}\text { FIRST } \\
\text { SECOND } \\
\text { THIRD } \\
\text { FOURTH }\end{array}$ & $\begin{array}{l}1 \\
1 \\
1 \\
0\end{array}$ & $\begin{array}{l}0.29 \\
0.65 \\
0.22 \\
0.00\end{array}$ & $\begin{array}{l}0.29 \\
0.40 \\
0.22 \\
0.00\end{array}$ & $\begin{array}{r}6.14 \\
12.01 \\
12.08 \\
0.00\end{array}$ & $\begin{array}{l}1.27 \\
1.35 \\
1.41 \\
0.00\end{array}$ & $\begin{array}{l}0.22 \\
0.32 \\
3.59 \\
0.00\end{array}$ & $\begin{array}{l}0.2 \bar{c} \\
0.32 \\
0.31 \\
0.00\end{array}$ & & \\
\hline \multicolumn{2}{|c|}{ SUMMARY } & 3 & 0.39 & 0.18 & 10.08 & 0.78 & 1.38 & 0.10 & & \\
\hline HR & $\begin{array}{l}\text { F IRST } \\
\text { SECOND } \\
\text { THIRD } \\
\text { FOURTH }\end{array}$ & $\begin{array}{l}0 \\
5 \\
6 \\
4\end{array}$ & $\begin{array}{l}0.00 \\
0.52 \\
0.47 \\
0.23\end{array}$ & $\begin{array}{l}0.00 \\
0.20 \\
0.19 \\
0.11\end{array}$ & $\begin{array}{l}0.09 \\
8.58 \\
5.39 \\
4.19\end{array}$ & $\begin{array}{l}0.00 \\
0.63 \\
0.89 \\
0.50\end{array}$ & $\begin{array}{l}0.00 \\
1.42 \\
0.31 \\
0.30\end{array}$ & $\begin{array}{l}0.00 \\
0.24 \\
0.11 \\
0.12\end{array}$ & $\begin{array}{l}0.45 \\
0.48\end{array}$ & $\begin{array}{l}0.04 \\
0.05\end{array}$ \\
\hline \multicolumn{2}{|c|}{ SUMMARY } & 15 & $0.4 \overline{3}$ & 0.11 & 6.65 & 0.41 & 0.80 & 0.12 & $0.4 \%$ & 0.03 \\
\hline \multicolumn{11}{|l|}{ CONTROL } \\
\hline ME & $\begin{array}{l}\text { FIRST } \\
\text { SECOND } \\
\text { THIRD } \\
\text { FOIRTH }\end{array}$ & $\begin{array}{l}1 \\
1 \\
1 \\
1\end{array}$ & $\begin{array}{l}0.51 \\
0.79 \\
0.16 \\
0.31\end{array}$ & $\begin{array}{l}0.30 \\
0.41 \\
0.16 \\
0.31\end{array}$ & $\begin{array}{l}4.19 \\
3.09 \\
1.14 \\
0.89\end{array}$ & $\begin{array}{l}0.93 \\
0.99 \\
1.14 \\
0.78\end{array}$ & $\begin{array}{l}0.52 \\
0.32 \\
0.23 \\
0.9 .1\end{array}$ & $\begin{array}{l}0.22 \\
0.32 \\
0.23 \\
0.21\end{array}$ & 0.41 & $\begin{array}{l}0.12 \\
0.11\end{array}$ \\
\hline \multicolumn{2}{|c|}{ SUMMARY } & 4 & 0.44 & 0.15 & 2.53 & 0.48 & 0.24 & $0.1 e$ & 0.50 & 0.09 \\
\hline $\mathrm{HF}$ & $\begin{array}{l}\text { FIRST } \\
\text { SECOND } \\
\text { THIRD } \\
\text { FOURTH }\end{array}$ & $\begin{array}{l}1 \\
1 \\
1\end{array}$ & $\begin{array}{l}0.50 \\
0.16 \\
0.20 \\
0.08\end{array}$ & $\begin{array}{l}0.29 \\
0.16 \\
0.19 \\
0.08\end{array}$ & $\begin{array}{l}2.68 \\
1.27 \\
2.41 \\
1.64\end{array}$ & $\begin{array}{l}0.85 \\
0.79 \\
0.84 \\
0.83\end{array}$ & $\begin{array}{l}0.22 \\
0.32 \\
0.23 \\
0.21\end{array}$ & $\begin{array}{l}0.22 \\
0.32 \\
0.23 \\
0.21\end{array}$ & $\begin{array}{l}0.91 \\
0.60\end{array}$ & $\begin{array}{l}0.13 \\
0.12\end{array}$ \\
\hline \multicolumn{2}{|c|}{ SUPPIARY } & ' & 0.ปّ" & 0.10 & 2.00 & $0: 41$ & 0.211 & 0.12 & 0.76 & 0.00 \\
\hline $\mathrm{HH}$ & $\begin{array}{l}\text { FIRST } \\
\text { ELCOHD } \\
\text { THIRO } \\
\text { FOURTH }\end{array}$ & $\begin{array}{l}1 \\
1 \\
1 \\
1\end{array}$ & $\begin{array}{l}0.16 \\
0.31 \\
0.16 \\
0.24\end{array}$ & $\begin{array}{l}0.16 \\
0.37 \\
0.16 \\
0.24\end{array}$ & $\begin{array}{l}3.05 \\
1.314 \\
2.52 \\
2.20\end{array}$ & $\begin{array}{l}0.90 \\
0.87 \\
0.91 \\
0.90\end{array}$ & $\begin{array}{l}0.22 \\
0.22 \\
0.23 \\
0.21\end{array}$ & $\begin{array}{l}0.22 \\
0.32 \\
0.23 \\
0.21\end{array}$ & $\begin{array}{l}0.47 \\
0.32\end{array}$ & $\begin{array}{l}0.13 \\
0.09\end{array}$ \\
\hline \multicolumn{2}{|c|}{ SUMMARY } & 4 & 0.22 & 0.11 & 2.28 & 0.44 & 0.24 & 0.12 & 0.40 & 0.08 \\
\hline ابث & $\begin{array}{l}\text { + IKG I } \\
\text { SECOND } \\
\text { THIRD } \\
\text { FOURTH }\end{array}$ & $\begin{array}{l}1 \\
1 \\
1\end{array}$ & $\begin{array}{l}0.29 \\
0.59 \\
0.20 \\
0.38\end{array}$ & $\begin{array}{l}0.29 \\
0.34 \\
0.20 \\
0.35\end{array}$ & $\begin{array}{l}3.19 \\
6.41 \\
6.69 \\
5.08\end{array}$ & $\begin{array}{l}118 \\
1.09 \\
1.13 \\
1.10\end{array}$ & $\begin{array}{l}0.8 p \\
0.32 \\
0.23 \\
0.25\end{array}$ & $\begin{array}{l}0.8 P \\
0.32 \\
0.23 \\
0.25\end{array}$ & $\begin{array}{l}0.82 \\
0.79\end{array}$ & $\begin{array}{l}0.14 \\
0.13\end{array}$ \\
\hline \multicolumn{2}{|c|}{ SLIMMARY } & 4 & 0.37 & 0.15 & 5.33 & 0.55 & 0.25 & 0.13 & 0.81 & 0.10 \\
\hline $\mathrm{HJ}$ & $\begin{array}{l}\text { FIRST } \\
\text { SFC.ONR } \\
\text { THIRD } \\
\text { FOURTH }\end{array}$ & $\begin{array}{l}0 \\
n \\
0 \\
1\end{array}$ & $\begin{array}{l}0.00 \\
0.06 \\
0.00 \\
0.21\end{array}$ & $\begin{array}{l}0.00 \\
0.00 \\
0.00 \\
0.2:\end{array}$ & $\begin{array}{l}0.00 \\
0.00 \\
0.00 \\
3.68\end{array}$ & $\begin{array}{l}0.00 \\
0.00 \\
0.00 \\
0.97\end{array}$ & $\begin{array}{l}0.00 \\
0.00 \\
0.00 \\
0.21\end{array}$ & $\begin{array}{l}0.00 \\
0.00 \\
0.00 \\
0.21\end{array}$ & 0.48 & 0.11 \\
\hline SUMMAR & & 1 & 0.21 & 0.21 & 3.68 & 0.97 & 0.21 & 0.21 & 0.48 & 0.11 \\
\hline
\end{tabular}

$\mathrm{nCI}=3.7 \times 10^{1} \mathrm{~Bq} . \quad \mathrm{pCI}=3.7 \times 10^{-2} \mathrm{~Bq}$.

Note: prefix $\mathrm{H}$ applied to factlitate sampling designation. 
1979, measurements of selected water quality and purity parameters at dowristream locations on the Peconic River and at control locations were initiated in order to provide some perspective on the same parameters in the Laboratory effluent (as reported in Table 13). These limited "grab" sample data are shown in Table 15. The effect of somewhat elevated levels of some of the parameters downstream results from other activities, residential and industrial, along the length of the Peconic River. This is based on the observation that the increased levels are not uniform but seem to be localized.

\subsubsection{Recharge Basin}

After use in "once through" heat exchangers and process cooling, about 19 million $1 \mathrm{~d}^{-1}$ (MLD) of water (data provided by Plant Engineering) was returned to the aquifer through on-site recharge basins: about 6.9 MLD to basin $N$ located about $610 \mathrm{~m}$ northeast of the AGS; about 5.8 MLD to bas in 0 about $670 \mathrm{~m}$ east of the HFBR; and about 6.3 MLD to basin P located $305 \mathrm{~m}$ south of the MRR (see Fig. 8). An organic phosphate is added to the AGS cooling and process water supply, which is independent of the Laboratory's potable supply, to establish a $\mathrm{PO}_{4}^{-3}$ concentration of about $2 \mathrm{ppm}$ in order to maintain the ambient iron in solution. Of the total AGS pumpage, about 4.3 MLD was discharged to the $N$ basin, and 4.3 MLD to the 0 basin. The HFBR secondary cooling system water recirculates through mechanical cooling towers and is treated to control corrosion and deposition of solids. Blowdown from this system, about 1.5 MLD, which contains about 6-8 ppm $\mathrm{PO}_{4}$ and 3-4 ppm benzotriazole is also discharged to the 0 sump. The MRR-MRC "once through" coolant which amounts to 6.3 MLD is not routinely treated and is discharged to the $P$ basin. Concentrations of radioactivity and other agents in the water discharged into these basins are monitored by routine weekly grab sampling. The average gross beta and tritium activity concentrations are shown in Table 16. The average gross beta activity concentration in the sump north of the AGS (N) and LINAC (T) are slightly above background since this water cools beam stops where activation products are formed and was $0.4 \%$ to $1 \%$ of the applicable RCG. However, in the Linac ( $T$ ) sump the gross beta activity was found to be about $1858 \mathrm{pCi} / 1$ in September. Investigations conducted at that time revealed that the increase in activity was due to a leak from the BLIP tank water which ultimately entered into the LINAC sump. The leak has been corrected and a significant amount of the contaminated water has been contained and will be disposed of in an appropriate manner. In the case of the sump east of the steam $p l a n t(U)$, the gross beta activity has continued to show levels approaching 3 to $8 \%$ of the RCG. However, wells used to monitor these sumps have not shown any increase in gross beta activity above background (gross beta activity in wells located upstream). Sampling frequency of these wells was increased in 1979 to investigate ground water contamination and no significant increases were noted in relation to previous years. Sampling frequency of the steam plant sump (U) will be increased in 1980 and analysis of these samples for specific radionuclides will be made. In general, the average concentration of gross beta and tritium activity concentrations in the other basins were slightly increased above those in the Laboratory supply wells and were about $0.1 \%$ of the applicable RCG for unidentified gross beta emitters and tritium. 
TABLE 15

1979 BNL ENVIRONMENTAL MONITORING DOWNSTREAM AND CONTROL WATER QUAL ITY AND PURITY

\begin{tabular}{|c|c|c|c|c|c|c|c|c|c|c|c|}
\hline LUL'A I IUN & $\checkmark$ SAMHLE & TEMPERATURE & $\mathrm{HH}$ & $\begin{array}{l}\text { OISSOLVED } \\
\text { OXYGEN } \\
\text { IHYM I }\end{array}$ & $\begin{array}{l}\text { CHLORIOES } \\
\text { (HIMI) }\end{array}$ & $\begin{array}{l}\text { NI TRATE } \\
\text { NI TROGEN } \\
\text { I PHMI }\end{array}$ & $\begin{array}{l}\text { TOTAL } \\
\text { PHOSPHOROUS } \\
\text { (HHM) }\end{array}$ & $\begin{array}{l}\text { DISSOLVED } \\
\text { SOLIDS } \\
\text { (HHM) }\end{array}$ & $\begin{array}{l}\text { CONDUC- } \\
\text { TIVITY } \\
\text { MILKUMHU/LM }\end{array}$ & $\begin{array}{l}\text { COL IFORM } \\
\text { FECAL } \\
\text {, /IUU ML। }\end{array}$ & $\begin{array}{c}\text { COL IFORM } \\
\text { TOTAL } \\
\text { (I IUU ML I }\end{array}$ \\
\hline HA & $\begin{array}{l}\text { MEAN } \\
\text { MINIMUM } \\
\text { MAXIMUM } \\
\text { NUMBER }\end{array}$ & $\begin{array}{r}14 \\
6 \\
21 \\
4\end{array}$ & $\begin{array}{l}5.7 \\
5.1 . \\
6.2 \\
4\end{array}$ & $\begin{array}{c}6.7 \\
1.8 \\
10.6 \\
4\end{array}$ & $\begin{array}{r}10.1 \\
8.6 \\
12: 1 \\
4\end{array}$ & $\begin{array}{l}0.59 \\
0.11 \\
0.45 \\
4\end{array}$ & $\begin{array}{l}0.07 \\
0.05 \\
0.10 \\
4\end{array}$ & $\begin{array}{r}55 \\
45 \\
64 \\
4\end{array}$ & $\begin{array}{r}63 \\
52 \\
69 \\
4\end{array}$ & $\begin{array}{r}35 \\
0 \\
100 \\
4\end{array}$ & $\begin{array}{r}175 \\
16 \\
392 \\
4\end{array}$ \\
\hline $\mathrm{HQT}$ & $\begin{array}{l}\text { MEAN } \\
\text { HINIPUII } \\
\text { MAXIMUM } \\
\text { NUMBER }\end{array}$ & $\begin{array}{r}12 \\
6 \\
18 \\
4\end{array}$ & $\begin{array}{l}.5 .6 \\
5.11 \\
6.0 \\
4\end{array}$ & $\begin{array}{r}7.0 \\
1.1 \\
12.0 \\
4\end{array}$ & $\begin{array}{r}9.9 \\
9.1 \\
11.8 \\
4\end{array}$ & $\begin{array}{l}0.30 \\
0.21 \\
0.41 \\
4\end{array}$ & $\begin{array}{l}0.06 \\
0.03 \\
0.14 \\
4\end{array}$ & $\begin{array}{r}57 \\
55 \\
59 \\
4\end{array}$ & $\begin{array}{r}52 \\
53 \\
67 \\
4\end{array}$ & $\begin{array}{r}29 \\
1 \\
68 \\
4\end{array}$ & $\begin{array}{r}144 \\
51 \\
300 \\
4\end{array}$ \\
\hline $\mathrm{HC}$ & $\begin{array}{l}\text { MEAN } \\
\text { MINIMUM } \\
\text { MAXIMUM } \\
\text { NUMBER }\end{array}$ & $\begin{array}{r}14 \\
6 \\
22 \\
4\end{array}$ & $\begin{array}{l}6.9 \\
5.5 \\
6.9 \\
4\end{array}$ & $\begin{array}{r}9.8 \\
0.6 \\
12.2 \\
4\end{array}$ & $\begin{array}{r}9.6 \\
9.1 \\
10.6 \\
4\end{array}$ & $\begin{array}{l}0.13 \\
0.04 \\
0.22 \\
4\end{array}$ & $\begin{array}{l}0.05 \\
0.02 \\
0.11 \\
4\end{array}$ & $\begin{array}{r}59 \\
51 \\
73 \\
4\end{array}$ & $\begin{array}{r}86 \\
54 \\
164 \\
4\end{array}$ & $\begin{array}{r}5 \\
0 \\
12 \\
12\end{array}$ & $\begin{array}{r}97 \\
32 \\
176 \\
4\end{array}$ \\
\hline HD & $\begin{array}{l}\text { MEAN } \\
\text { MINIMUM } \\
\text { MAXIMUM } \\
\text { NUMBER }\end{array}$ & $\begin{array}{r}13 \\
6 \\
21 \\
4\end{array}$ & $\begin{array}{l}6.2 \\
5.6 \\
6.6 \\
4\end{array}$ & $\begin{array}{r}10.6 \\
8.2 \\
12.6 \\
4\end{array}$ & $\begin{array}{r}12.0 \\
9.1 \\
19.2 \\
4\end{array}$ & $\begin{array}{l}0.31 \\
0.08 \\
0.64 \\
3\end{array}$ & $\begin{array}{l}0.04 \\
0.01 \\
0.09 \\
4\end{array}$ & $\begin{array}{r}72 \\
47 \\
116 \\
3\end{array}$ & $\begin{array}{r}101 \\
56 \\
172 \\
4\end{array}$ & $\begin{array}{r}35 \\
0 \\
104 \\
4\end{array}$ & $\begin{array}{r}127 \\
10 \\
190 \\
3\end{array}$ \\
\hline $\mathrm{HF}$ & $\begin{array}{l}\text { MEAN } \\
\text { MINIMUM } \\
\text { MAXIMUM } \\
\text { NUMBER }\end{array}$ & $\begin{array}{r}14 \\
5 \\
23 \\
4\end{array}$ & $\begin{array}{l}5.3 \\
4.5 \\
5.7 \\
4\end{array}$ & $\begin{array}{r}6.8 \\
1.4 \\
10.4 \\
4\end{array}$ & $\begin{array}{l}6.9 \\
6.6 \\
7.1 \\
4\end{array}$ & $\begin{array}{l}0.21 \\
0.03 \\
0.67 \\
4\end{array}$ & $\begin{array}{l}0.04 \\
0.01 \\
0.10 \\
4\end{array}$ & $\begin{array}{r}44 \\
36 \\
52 \\
4\end{array}$ & $\begin{array}{r}45 \\
42 \\
48 \\
4\end{array}$ & $\begin{array}{r}28 \\
0 \\
104 \\
4\end{array}$ & $\begin{array}{r}133 \\
10 \\
270 \\
4\end{array}$ \\
\hline $\mathrm{HH}$ & $\begin{array}{l}\text { MEAN } \\
\text { MINIMUM } \\
\text { MAXIMUM } \\
\text { NUMBER }\end{array}$ & $\begin{array}{r}13 \\
5 \\
21 \\
4\end{array}$ & $\begin{array}{l}5.3 \\
6.8 \\
5.4 \\
4\end{array}$ & $\begin{array}{r}10.8 \\
6.6 \\
12.6 \\
4\end{array}$ & $\begin{array}{r}13.0 \\
9.6 \\
19.2\end{array}$ & $\begin{array}{l}1.09 \\
0.90 \\
1.32 \\
4\end{array}$ & $\begin{array}{l}0.02 . \\
0.01 \\
0.04 \\
4\end{array}$ & $\begin{array}{r}84 \\
70 \\
110 \\
.4\end{array}$ & $\begin{array}{r}106 \\
94 \\
134 \\
4\end{array}$ & $\begin{array}{r}17 \\
46 \\
4\end{array}$ & $\begin{array}{r}58 \\
10 \\
100 \\
4\end{array}$ \\
\hline HI & $\begin{array}{l}\text { MEAN } \\
\text { MINIMUM } \\
\text { MAXIMUM } \\
\text { NUMBER }\end{array}$ & $\begin{array}{r}14 \\
4 \\
211 \\
4\end{array}$ & $\begin{array}{l}6.6 \\
6.3 \\
C .0 \\
4\end{array}$ & $\begin{array}{r}10.3 \\
8.4 \\
12.6 \\
4\end{array}$ & $\begin{array}{l}19.5 \\
18.2 \\
21.7 \\
4\end{array}$ & $\begin{array}{l}0.17 \\
0.10 \\
0.31 \\
4\end{array}$ & $\begin{array}{l}0.02 \\
0.01 \\
0.03 \\
4\end{array}$ & $\begin{array}{l}74 \\
60 \\
911 \\
4\end{array}$ & $\begin{array}{r}105 \\
90 \\
112 \\
4\end{array}$ & $\begin{array}{r}67 \\
5 \\
100 \\
4\end{array}$ & $\begin{array}{r}115 \\
22 \\
210 \\
4\end{array}$ \\
\hline HJ & $\begin{array}{l}\text { MEAN } \\
\text { MINIMUM } \\
\text { MAXIMUM } \\
\text { NUMBER }\end{array}$ & $\begin{array}{r}3 \\
3 \\
-3 \\
1\end{array}$ & $\begin{array}{l}7.0 \\
7.0 \\
7.0 \\
1\end{array}$ & $\begin{array}{l}12.4 \\
12.4 \\
12.4 \\
1\end{array}$ & $\begin{array}{l}9.1 \\
9.1 \\
9.1 \\
1\end{array}$ & $\begin{array}{l}0.38 \\
0.38 \\
0.38 \\
1 .\end{array}$ & $\begin{array}{l}0.04 \\
0.04 \\
0.04 \\
1\end{array}$ & $\begin{array}{r}52 \\
52 \\
52 \\
1\end{array}$ & $\begin{array}{c}68 \\
68 \\
68 \\
1\end{array}$ & $\begin{array}{l}0 \\
0 \\
0 \\
1\end{array}$ & $\begin{array}{l}0 \\
0 \\
0 \\
1\end{array}$ \\
\hline $\mathrm{HL}$ & $\begin{array}{l}\text { MEAN } \\
\text { MINIMUM } \\
\text { MAXIMUM } \\
\text { NUMBER }\end{array}$ & $\begin{array}{r}17 \\
6 \\
25 \\
3\end{array}$ & $\begin{array}{l}5.4 \\
4.9 \\
6.0 \\
3\end{array}$ & $\begin{array}{l}7.6 \\
6.2 \\
9.2 \\
3\end{array}$ & $\begin{array}{l}19.1 \\
12.6 \\
12.4 \\
3\end{array}$ & $\begin{array}{l}3.55 \\
1.15 \\
7.40 \\
3\end{array}$ & $\begin{array}{l}0.35 \\
0.15 \\
0.111 \\
3\end{array}$ & $\begin{array}{r}92 \\
62 \\
126 \\
3\end{array}$ & $\begin{array}{r}112 \\
73 \\
156 \\
3\end{array}$ & $\begin{array}{l}0 \\
0 \\
0 \\
3\end{array}$ & $\begin{array}{r}133 \\
120 \\
145 \\
2\end{array}$ \\
\hline HR & $\begin{array}{l}\text { HE ANI } \\
\text { MINIMU: } \\
\text { MAXIMUM } \\
\text { NUMELR }\end{array}$ & $\begin{array}{r}17 \\
5 \\
26 \\
17\end{array}$ & $\begin{array}{r}6.1 \\
5.3 \\
6.6 \\
17\end{array}$ & $\begin{array}{r}8.8 \\
7.4 \\
11.6 \\
17\end{array}$ & $\begin{array}{l}1 E .0 \\
9.0 \\
16.1 \\
0\end{array}$ & $\begin{array}{l}0.35 \\
0.03 \\
0.81 \\
0\end{array}$ & $\begin{array}{l}0.00 \\
0.05 \\
0.19 \\
0\end{array}$ & $\begin{array}{r}80 \\
60 \\
189 \\
8\end{array}$ & $\begin{array}{r}90 \\
74 \\
185 \\
17\end{array}$ & $\begin{array}{r}10 \\
1 \\
358 \\
16\end{array}$ & $\begin{array}{r}r 19 \\
10 \\
950 \\
16\end{array}$ \\
\hline
\end{tabular}


TABLE 15 (Continued)

1979 BNL ENVIRONMENTAL MONITORING DOWNSTREAM AND CONTROL WATER OUALITY AND PURITY-MET

\begin{tabular}{|c|c|c|c|c|c|c|c|c|}
\hline & & \multicolumn{7}{|c|}{ METALS (IN PPM) } \\
\hline & & $A G$ & $C D$ & CR & $\mathrm{CU}$ & $\mathrm{Fe}$ & $\mathrm{PB}$ & $\mathrm{ZN}$ \\
\hline HA & $\begin{array}{l}\text { MEAN } \\
\text { MINIMUM } \\
\text { MAXIMUM } \\
\text { EXCEPTION } \\
\text { NUMBER }\end{array}$ & $\begin{array}{l}.00 ! \\
.00 ! \\
.001 \\
3^{4}\end{array}$ & $\begin{array}{l}.0004 \\
.0002 \\
.0005 \\
4 \\
4\end{array}$ & $\begin{array}{l}.009 \\
.002 \\
.028 \\
3 \\
4\end{array}$ & $\begin{array}{l}.006 \\
.003 \\
.015 \\
0 \\
4\end{array}$ & $\begin{array}{l}.859 \\
.310 \\
2.250 \\
0 \\
4\end{array}$ & $\begin{array}{l}.007 \\
.002 \\
.017 \\
3 \\
4\end{array}$ & $\begin{array}{l}.012 \\
.004 \\
.022 \\
0 \\
4\end{array}$ \\
\hline $\mathrm{HB}$ & $\begin{array}{l}\text { MEAN } \\
\text { MINIMUM } \\
\text { MAXIMUM } \\
\text { EXCEPTION } \\
\text { NUMBER }\end{array}$ & $\begin{array}{l}.001 \\
.001 \\
.001 \\
3 \\
4\end{array}$ & $\begin{array}{l}.0004 \\
.0002 \\
.0006 \\
4^{4}\end{array}$ & $\begin{array}{l}.002 \\
.00 ! \\
.002 \\
4 \\
4\end{array}$ & $\begin{array}{l}.003 \\
.002 \\
.004 \\
0 \\
4\end{array}$ & $\begin{array}{l}1.539 \\
.310 \\
3.600 \\
0 \\
4\end{array}$ & $\begin{array}{l}.003 \\
.002 \\
.005 \\
4 \\
4\end{array}$ & $\begin{array}{l}.010 \\
.003 \\
.018 \\
0 \\
4\end{array}$ \\
\hline $\mathrm{HC}$ & $\begin{array}{l}\text { MEAN } \\
\text { MINIMUM } \\
\text { MAXIMUM } \\
\text { EXCEPT ION } \\
\text { NUMBER }\end{array}$ & $\begin{array}{l}.001 \\
.001 \\
.001 \\
4 \\
4\end{array}$ & $\begin{array}{l}.0004 \\
.0002 \\
.0006 \\
4 \\
4\end{array}$ & $\begin{array}{l}.002 \\
.001 \\
.002 \\
4 \\
4\end{array}$ & $\begin{array}{l}.002 \\
.001 \\
i 002 \\
i \\
4\end{array}$ & $\begin{array}{l}.907 \\
.380 \\
1.980 \\
0 \\
4\end{array}$ & $\begin{array}{l}.004 \\
.002 \\
.005 \\
4 \\
4\end{array}$ & $\begin{array}{l}.006 \\
.002 \\
.013 \\
0 \\
4\end{array}$ \\
\hline HD & $\begin{array}{l}\text { MEAN } \\
\text { MINIMUM } \\
\text { MAXIMUM } \\
\text { EXCEPTION } \\
\text { NUMBER }\end{array}$ & $\begin{array}{l}.001 \\
.001 \\
.001 \\
4 \\
4\end{array}$ & $\begin{array}{l}.0005 \\
.0003 \\
.0006 \\
3 \\
4\end{array}$ & $\begin{array}{l}.002 \\
.001 \\
.002 \\
4 \\
4\end{array}$ & $\begin{array}{l}.003 \\
.001 \\
.006 \\
0 \\
4\end{array}$ & $\begin{array}{l}.655 \\
.285 \\
1.310 \\
0 \\
4\end{array}$ & $\begin{array}{l}.004 \\
.002 \\
.005 \\
4 \\
4\end{array}$ & $\begin{array}{l}.005 \\
.002 \\
.011 \\
0 \\
4\end{array}$ \\
\hline $\mathrm{HF}$ & $\begin{array}{l}\text { MEAN } \\
\text { MINIMUM } \\
\text { MAXIMUM } \\
\text { EXCEPTION } \\
\text { NUHABER }\end{array}$ & $\begin{array}{l}.001 \\
.001 \\
.001 \\
4 \\
4\end{array}$ & $\begin{array}{l}.0004 \\
.0002 \\
.0006 \\
4 \\
4\end{array}$ & $\begin{array}{l}.002 \\
.001 \\
.002 \\
4 \\
4\end{array}$ & $\begin{array}{l}.001 \\
.001 \\
.002 \\
i \\
4\end{array}$ & $\begin{array}{c}1.689 \\
.255 \\
5.250 \\
0 \\
4\end{array}$ & $\begin{array}{l}.004 \\
.002 \\
.005 \\
3 \\
4\end{array}$ & $\begin{array}{l}.005 \\
.002 \\
.007 \\
0 \\
4\end{array}$ \\
\hline $\mathrm{HH}$ & $\begin{array}{l}\text { MEAN } \\
\text { M!!:IMUM } \\
\text { ridXIMUM } \\
\text { EXCEPT ION } \\
\text { NUMBER }\end{array}$ & $\begin{array}{l}.001 \\
.001 \\
.001 \\
4 \\
4\end{array}$ & $\begin{array}{l}.0004 \\
.0002 \\
.0006 \\
4 \\
4\end{array}$ & $\begin{array}{l}.002 \\
.001 \\
.002 \\
4 \\
4\end{array}$ & $\begin{array}{l}.001 \\
.001 \\
.002 \\
! \\
4\end{array}$ & $\begin{array}{l}.157 \\
.015 \\
.267 \\
i \\
4\end{array}$ & $\begin{array}{l}.004 \\
.002 \\
.005 \\
4 \\
4\end{array}$ & $\begin{array}{l}.002 \\
.001 \\
.003 \\
2^{4}\end{array}$ \\
\hline HI & $\begin{array}{l}\text { MEAN } \\
\text { MINIMUM } \\
\text { MAXIMUM } \\
\text { EXCEPT ION } \\
\text { NUMBER }\end{array}$ & $\begin{array}{l}.001 \\
.001 \\
.001 \\
4 \\
4\end{array}$ & $\begin{array}{l}.0004 \\
.0002 \\
.0006 \\
4 \\
4\end{array}$ & $\begin{array}{l}.004 \\
.002 \\
.011 \\
3 \\
4\end{array}$ & $\begin{array}{l}.002 \\
.002 \\
.002 \\
0 \\
4\end{array}$ & $\begin{array}{l}.046 \\
.046 \\
.046 \\
0 \\
1\end{array}$ & $\begin{array}{l}.008 \\
.004 \\
.015 \\
i \\
4\end{array}$ & $\begin{array}{l}.005 \\
.002 \\
.009 \\
0 \\
4\end{array}$ \\
\hline$H_{v}$ & $\begin{array}{l}\text { MEAN } \\
\text { MINIMUM } \\
\text { MAXIMUM } \\
\text { EXCEPTION } \\
\text { NUMBER }\end{array}$ & $\begin{array}{l}.001 \\
.001 \\
i^{001}\end{array}$ & $\begin{array}{l}.0002 \\
.0002 \\
i 0002 \\
i\end{array}$ & $\begin{array}{l}.002 \\
.002 \\
.002 \\
i \\
1\end{array}$ & $\begin{array}{l}.002 \\
.002 \\
.002 \\
0 \\
1\end{array}$ & $\begin{array}{l}.076 \\
.075 \\
.076 \\
0 \\
1\end{array}$ & $\begin{array}{l}.002 \\
.002 \\
i .002 \\
i\end{array}$ & $\begin{array}{l}.013 \\
.013 \\
.013 \\
0 \\
1\end{array}$ \\
\hline HL & $\begin{array}{l}\text { MEAN } \\
\text { MINIMUMA } \\
\text { MAXIMUM } \\
\text { EXCEPT ION } \\
\text { NUMBER }\end{array}$ & $\begin{array}{l}.002 \\
.001 \\
.003 \\
2 \\
3\end{array}$ & $\begin{array}{l}.0008 \\
.0003 \\
.0013 \\
1^{3}\end{array}$ & $\begin{array}{l}.002 \\
.001 \\
.002 \\
3 \\
3\end{array}$ & $\begin{array}{l}.027 \\
.007 \\
.053 \\
0 \\
3\end{array}$ & $\begin{array}{r}.870 \\
.400 \\
1.610 \\
0 \\
3\end{array}$ & $\begin{array}{l}.008 \\
.005 \\
i^{.14} \\
3\end{array}$ & $\begin{array}{l}.054 \\
.009 \\
090 \\
0 \\
3\end{array}$ \\
\hline HR & $\begin{array}{l}\text { MEAN } \\
\text { MINIMUM } \\
\text { MAXIMUM } \\
\text { EXCEPT ION } \\
\text { NUMBER }\end{array}$ & $\begin{array}{l}.001 \\
.001 \\
.001 \\
3 \\
3\end{array}$ & $\begin{array}{l}.0005 \\
.0003 \\
.0006 \\
3 \\
3\end{array}$ & $\begin{array}{l}.002 \\
.001 \\
.002 \\
3 \\
3\end{array}$ & $\begin{array}{l}.002 \\
.001 \\
i^{.002} \\
3\end{array}$ & $\begin{array}{l}.597 \\
.1405 \\
.767 \\
0 \\
3\end{array}$ & $\begin{array}{l}.004 \\
.002 \\
.005 \\
.3 \\
3\end{array}$ & $\begin{array}{l}.004 \\
.001 \\
.008 \\
0 \\
3\end{array}$ \\
\hline
\end{tabular}

Reference Standard - Table 29

Note: prefix $\mathrm{H}$ applied to

facilitate sampling designation. 
TABLE 16

1979 BNL MONTHLY SUMP SAMPLES

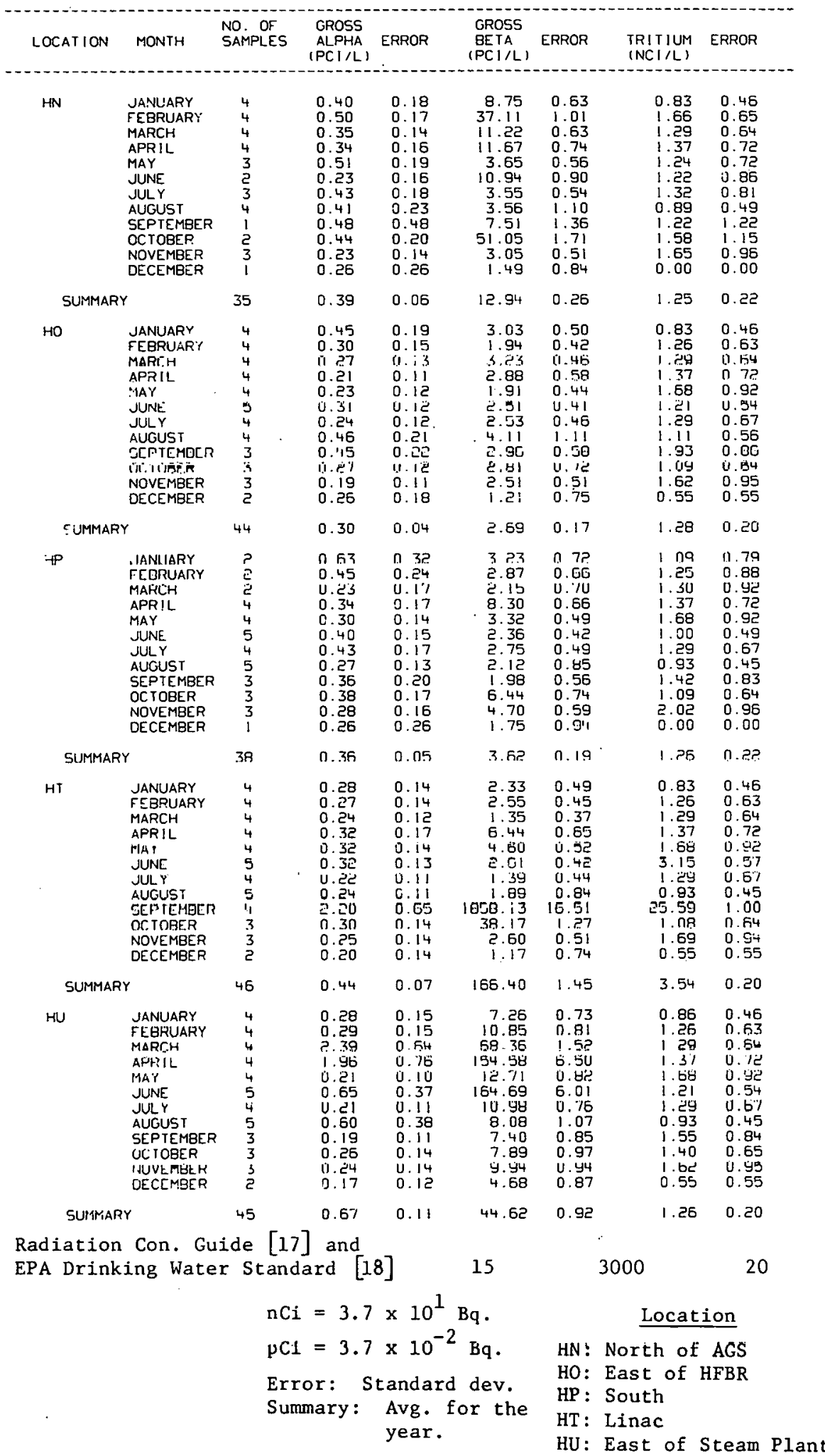


Water quality data obtained during 1979 from periodic (approximately monthly) analyses of "grab" samples from the recharge basins, from a culvert which conducts some air conditioning tower blowdown and storm sewer influents from the southeast Laboratory building complex to a natural sump south of the warehouse area (about $1.2 \mathrm{~km}$ south of Building 610 , see Fig. 3) is shown in Table 17. Al1 were within established standards for ground water quality except at sump HU where washout from recharging of ion exchange resins (used in softening water) increased the concentrations of cations and anions, thereby increasing conductivity. Excess metal concentrations, such as for $\mathrm{Cd}, \mathrm{Cr}, \mathrm{Cu}$, $\mathrm{Fe}, \mathrm{Pb}$ and $\mathrm{Zn}$, indicate effects of chemical treatment for keeping iron in solution and steam plant condensates and boiler washings ( $\mathrm{Pb}$ and $\mathrm{Zn}$ from pipes). Investigations are in progress to pinpoint the sources of these elements and examine the area in the vicinity of this sump for possible location of cesspools and/or irregularities in the sanitary system.

\subsubsection{Aquatic Biological Studies}

During the summers of 1977,1978 , and 1979, a comprehensive program aimed at understanding the effect of the Laboratory effluent on the Peconic River biological system over the years was initiated. This study, which is expected to take three to four years, will be phased in gradually. In 1977, 1978 and 1979, the program was exploratory, wherein sampling stations, sampling techniques and analytical procedures and their limitations were investigated. The results so obtained will be used to develop an adequate program to be implemented in 1981 when it is hoped that the data so obtained would be amenable to modeling for predictive purposes. Additional data from previous years will also be used. Figure 7 indicates some of the sampling locations. These are:

$$
\begin{aligned}
& \text { E - Reference point-headwaters of the Peconic River-Control Station, } \\
& \text { K }-0.045 \mathrm{~km} \text {, no vegetation, significant influence of chlorine. } \\
& \text { L }-0.106 \mathrm{~km} \text {, } \\
& \text { M }-0.798 \mathrm{~km} \text {, referred to as the former site boundary, } \\
& \text { Q }-2.11 \mathrm{~km} \text {, the present site boundary, fish collected from here, } \\
& \text { A }-4.85 \mathrm{~km} \text {, } \\
& \text { S }-7.05 \mathrm{~km}, \\
& \text { T }-10.82 \mathrm{~km} \text {, } \\
& \text { II }-14.23 \mathrm{~km} \\
& \text { W }-18.18 \mathrm{~km} \text {, mussel bed, } \\
& \text { Y }-22.21 \mathrm{~km} \text {, salt water meets fresh water. }
\end{aligned}
$$


TABLE 17

1979 GNL ENVIRONMENTAL MONITORING RECHARGE BASINS WATER OUALITY AND PURITY

\begin{tabular}{|c|c|c|c|c|c|c|c|c|c|c|c|}
\hline LOCATION & N SAMPLE & TEMPERATURE & $\mathrm{PH}$ & $\begin{array}{l}\text { OISSOLVED } \\
\text { OXYGEN } \\
\text { (PPM) }\end{array}$ & $\begin{array}{l}\text { CHLORIDES } \\
\text { (PPM) }\end{array}$ & $\begin{array}{l}\text { NI TRATE } \\
\text { NITROGEN } \\
\text { (PPM) }\end{array}$ & $\begin{array}{l}\text { TOTAL } \\
\text { PHOSPHOROUS } \\
\text { (PPM) }\end{array}$ & $\begin{array}{l}\text { DISSOL VED } \\
\text { SOL IDS } \\
\text { (PPM) }\end{array}$ & $\begin{array}{l}\text { CONDUC- } \\
\text { TIVITY } \\
\text { MICROMHO/CM }\end{array}$ & $\begin{array}{c}\text { COL IFORM } \\
\text { FECAL } \\
(1100 \mathrm{ML})\end{array}$ & $\begin{array}{c}\text { COL (FORM } \\
\text { TOTAL } \\
(1100 \mathrm{ML})\end{array}$ \\
\hline $\mathbf{H N}$ & $\begin{array}{l}\text { MEAN } \\
\text { MINIMUM } \\
\text { MAXIKIUM } \\
\text { NUMBER }\end{array}$ & $\begin{array}{r}15 \\
8 \\
26 \\
35\end{array}$ & $\begin{array}{r}6.5 \\
5.0 \\
7.2 \\
35\end{array}$ & $\begin{array}{r}9.6 \\
8.2 \\
11.2 \\
35\end{array}$ & $\begin{array}{r}15.0 \\
0.0 \\
20.2 \\
10\end{array}$ & $\begin{array}{l}0.21 \\
0.08 \\
0.46 \\
10\end{array}$ & $\begin{array}{l}0.19 \\
0.02 \\
0.58 \\
10\end{array}$ & $\begin{array}{l}72 \\
65 \\
86 \\
9\end{array}$ & $\begin{array}{r}102 \\
64 \\
215 \\
35\end{array}$ & $\begin{array}{l}1 \\
0 \\
4 \\
5\end{array}$ & $\begin{array}{r}98 \\
14 \\
210 \\
5\end{array}$ \\
\hline MO & $\begin{array}{l}\text { MEAN } \\
\text { MINIMUM } \\
\text { MAXIMUM } \\
\text { NUMBER }\end{array}$ & $\begin{array}{r}16 \\
9 \\
27 \\
44\end{array}$ & $\begin{array}{r}6.6 \\
5.7 \\
7.4 \\
44\end{array}$ & $\begin{array}{r}9.7 \\
9.0 \\
12.0 \\
44\end{array}$ & $\begin{array}{l}19.2 \\
15.6 \\
23.2 \\
11\end{array}$ & $\begin{array}{l}0.25 \\
0.03 \\
0.65 \\
11\end{array}$ & $\begin{array}{l}0.22 \\
0.01 \\
0.57 \\
11\end{array}$ & $\begin{array}{r}70 \\
53 \\
77 \\
9\end{array}$ & $\begin{array}{r}114 \\
88 \\
165 \\
44\end{array}$ & $\begin{array}{l}1 \\
0 \\
3 \\
5\end{array}$ & $\begin{array}{r}50 \\
0 \\
120 \\
5\end{array}$ \\
\hline$H$ & $\begin{array}{l}\text { MEAN } \\
\text { MINIMUM } \\
\text { MAXIMUM } \\
\text { NUMBER }\end{array}$ & $\begin{array}{l}15 \\
11 \\
28 \\
38\end{array}$ & $\begin{array}{r}6.1 \\
5.3 \\
7.0 \\
38\end{array}$ & $\begin{array}{r}6.5 \\
0.6 \\
13.2 \\
=0\end{array}$ & $\begin{array}{l}29.5 \\
23.7 \\
34.8 \\
.11\end{array}$ & $\begin{array}{l}1.43 \\
0.91 \\
1.72\end{array}$ & $\begin{array}{l}0.02 \\
0.01 \\
0.0 ! 1 \\
11\end{array}$ & $\begin{array}{r}115 \\
104 \\
133 \\
11\end{array}$ & $\begin{array}{r}176 \\
151 \\
280 \\
38\end{array}$ & $\begin{array}{l}0 \\
0 \\
n \\
3\end{array}$ & $\begin{array}{l}1 \\
0 \\
4 \\
3\end{array}$ \\
\hline rrs & $\begin{array}{l}\text { MEANI } \\
\text { MINIMUM } \\
\text { MAXIMUM } \\
\text { NUMBER }\end{array}$ & $\begin{array}{r}16 \\
8 \\
26 \\
50\end{array}$ & $\begin{array}{l}6.7 \\
5.4 \\
9.8 \\
50\end{array}$ & $\begin{array}{l}9.4 \\
7.0 \\
10.8 \\
50\end{array}$ & $\begin{array}{l}23.3 \\
5.5 \\
42.3 \\
49\end{array}$ & $\begin{array}{l}0.74 \\
0.08 \\
10.94 \\
49\end{array}$ & $\begin{array}{l}0.2 \theta \\
\text { U. Ũ } \\
1.96 \\
48\end{array}$ & $\begin{array}{r}111 \\
78 \\
213 \\
9\end{array}$ & $\begin{array}{r}139 \\
18 \\
380 \\
50\end{array}$ & $\begin{array}{r}63 \\
0 \\
336 \\
45\end{array}$ & $\begin{array}{r}242 \\
0 \\
890 \\
41\end{array}$ \\
\hline HI & $\begin{array}{l}\text { MEAN } \\
\text { HINIMU! I I I } \\
\text { MAXIMUM } \\
\text { NUMEER }\end{array}$ & $\begin{array}{l}19 \\
12 \\
31 \\
45\end{array}$ & $\begin{array}{l}6.7 \\
5.7 \\
7.6 \\
45\end{array}$ & $\begin{array}{l}8.8 \\
5.6 \\
10.6 \\
45\end{array}$ & $\begin{array}{l}22.5 \\
15.5 \\
28.1 \\
11\end{array}$ & $\begin{array}{l}0.39 \\
0.12 \\
0.67 \\
11\end{array}$ & $\begin{array}{l}0.04 \\
0.01 \\
0.11 \\
11\end{array}$ & $\begin{array}{r}98 \\
80 \\
139 \\
9\end{array}$ & $\begin{array}{r}130 \\
100 \\
160 \\
45\end{array}$ & $\begin{array}{l}0 \\
0 \\
0 \\
5\end{array}$ & $\begin{array}{l}2 \\
0 \\
\mathbf{0} \\
5\end{array}$ \\
\hline HU & $\begin{array}{l}\text { MEAN } \\
\text { MINIMUM } \\
\text { MAXIMUM } \\
\text { NUMEEA }\end{array}$ & $\begin{array}{l}21 \\
1 \\
32 \\
44\end{array}$ & $\begin{array}{r}9.8 \\
2.7 \\
11.3 \\
44\end{array}$ & $\begin{array}{l}6.6 \\
2.6 \\
10.8 \\
39\end{array}$ & $\begin{array}{r}976.2 \\
27.7 \\
.00 .0 \\
12\end{array}$ & $\begin{array}{r}0.88 \\
0.24 \\
2.25 \\
1 ? .25\end{array}$ & $\begin{array}{l}0.34 \\
0.01 \\
0.71 \\
12\end{array}$ & $\begin{array}{r}19 月 3 \\
190 \\
6562 \\
10\end{array}$ & $\begin{array}{r}1436 \\
153 \\
+\quad 0 \\
44\end{array}$ & $\begin{array}{r}7 \\
0 \\
40 \\
6\end{array}$ & $\begin{array}{r}13 \\
0 \\
80 \\
5\end{array}$ \\
\hline
\end{tabular}

1979 BNL ENYIRONMENTAL MONITORING RECHARGE BASINS WATER QUALITY AND PURITY-METALS

\begin{tabular}{|c|c|c|c|c|c|c|c|c|}
\hline & & \multicolumn{7}{|c|}{ METALS ! [N] PPM] } \\
\hline & & AO & $C D$ & in & $\mathrm{Cu}$ & FE. & PQ & $Z N$ \\
\hline HS & $\begin{array}{l}\text { MEAN } \\
\text { MINIMUM } \\
\text { MAXXIMUM } \\
\text { EXCEPTION } \\
\text { NUMBER }\end{array}$ & $\begin{array}{l}.001 \\
.001 \\
i .001 \\
i\end{array}$ & $\begin{array}{l}.0006 \\
.0006 \\
.0006 \\
i\end{array}$ & $\begin{array}{l}.002 \\
.002 \\
i^{202} \\
1\end{array}$ & $\begin{array}{l}.013 \\
.013 \\
013 \\
0 \\
1 .\end{array}$ & $\begin{array}{l}2.460 \\
2.460 \\
2.460 \\
0 \\
1\end{array}$ & $\begin{array}{l}.005 \\
.005 \\
i \\
i \\
1\end{array}$ & $\begin{array}{l}.197 \\
.197 \\
197 \\
0 \\
1\end{array}$ \\
\hline HU & $\begin{array}{l}\text { MEAN } \\
\text { MINIMUM } \\
\text { MAXIMIIM } \\
\text { EREEPTION } \\
\text { NUMBER }\end{array}$ & $\begin{array}{l}.001 \\
.007 \\
i^{007} \\
1\end{array}$ & $\begin{array}{l}1.5200 \\
1.5200 \\
1.5200 \\
0 \\
1\end{array}$ & $\begin{array}{l}\text { 8. } 100 \\
0.100 \\
y .100 \\
0 \\
1\end{array}$ & $\begin{array}{l}10.100 \\
10.100 \\
16.100 \\
u \\
1\end{array}$ & $\begin{array}{c}717.000 \\
747.000 \\
747.000 \\
i\end{array}$ & $\begin{array}{l}5.320 \\
5.320 \\
5.320 \\
0 \\
1\end{array}$ & $\begin{array}{c}05.600 \\
95.600 \\
95.600 \\
i \\
i\end{array}$ \\
\hline
\end{tabular}

Beference Standaris - Tahle 29

HN : North of AGS

HO: East of HFBR

HP: South of MRR

HS: South of Warehouse

HT: North of LINAC

HU: Easl uf Sleall Plaml

Exception: Below Minimum Detection Limit (MDL; 
These locations were sampled for sediment and vegetation common to most of the sites. A significant improvement from previous sediment sampling techniques was the use of coring to collect river bottom sediments. This procedure will enable determination of both vertical and horizontal migration of radionuclides in the bottom sediments. Fish samples were limited by availability to Station Q. Mussel samples were available only from Stations $T$ and $W$. Intensive sampling of the aquatic environment will be done in 1980 and 1981 , especially for stations beyond the influence of the Peconic River.

The data in Table 18 are restricted to ${ }^{90} \mathrm{Sr}$ and ${ }^{137} \mathrm{Cs}$. These radionuclides were found in detectable concentrations above the MDL and can be considered as principal contributors to body burden estimates in man. Other nuclides such as $60 \mathrm{Co}$, which are attributable to Laboratory effluents, were present but were either less than or equal to the MDL for the counting system used (Table 29 and Appendix B). In analyzing the data, it was noted that beyond Station $Q$, most of the radionuclides were at or below MDL. Also, since Station $Q$ represents the site boundary, a proper documentation of all releases is critical for the evaluation of Laboratory releases. The table, therefore, is also restricted to Station $Q$ on the above basis.

Looking at the concentration factors on a unit weight basis for ${ }^{90} \mathrm{Sr}$ and ${ }^{137} \mathrm{Cs}$ at Station $Q$, it is noted that there is an increase in unit activity across the food chain: water-vegetation-fish. For ${ }^{90} \mathrm{Sr}$, the concentration factor for fish ranges from 69 to 103 in flesh. In the case of $137 \mathrm{Cs}$, the concentration factor for fish ranged from 1190-1280. These results are in accordance with observations in aquatic environments $(22,23)$. Using an assumed intake of $1.36 \mathrm{~kg} / \mathrm{yr}$ of fish flesh (edible portions) for persons fishing along the Peconic River and the indicated range of concentrations of $90 \mathrm{Sr}$ and $137 \mathrm{Cs}$ in fish flesh (edible portions), one can compute for an adult, body burdens for these radionuclides in man to be ranging from $0.05 \%$ to $0.15 \%$ respectively of the doses permissible by the Regulatory Guide (24).

\subsubsection{Survei11ance We11s}

\subsubsection{Potable Water and Process Supply Wells}

The Laboratory's potable water wells and cooling water supply wells are screened at a depth of about $30 \mathrm{~m}$, or about $15 \mathrm{~m}$ below the water table, in the Long Island surface layer of glacial outwash, sand and gravel. These wells (Fig. 8) are located generally west to northwest of the Laboratory's principal facilities and 'upstream' of the local ground water flow pattern. An average of about 28.4 MLD was pumped from them.

Bimonthly grab samples were obtained from these wells. These were analyzed for gross alpha, gross beta and tritium. All gross alpha concentratïons were $<1 \mathrm{pCi} / 1$ iter $\left(<1 \times 10^{-9} \mu \mathrm{Ci} \mathrm{ml}-1\right.$ or $\left.3.7 \times 10^{-5} \mathrm{~Bq} \mathrm{~m} l^{-1}\right)$, and almost al1 tritium concentrations were $<1.0 \mathrm{nCi} / 1$ iter $\left(<10^{-6} \mu \mathrm{Ci} / \mathrm{ml}\right.$ or $3.7 \times 10^{-2} \mathrm{~Bq}$ $\left.\mathrm{ml}^{-1}\right)$. The gross beta and tritium results are 1 isted in Table 19. There are some differences in the gross beta concentrations among these we1ls. However, these differences are not corraborated by tritium concentrations indicating that the sources of gross beta are not related to Laboratory activities. In general, 
TABLE 18

1979 BNL Environmental Monitoring Concentration of ${ }^{90} \mathrm{Sr}$ and ${ }^{137} \mathrm{Cs}$ in Water, Sediment, Vegetation and Fish Obtained from the Peconic River at the Site Perimeter as Observed During 19\%4-19\%y

\begin{tabular}{|c|c|c|c|c|c|c|c|c|}
\hline \multirow{3}{*}{ Year } & \multicolumn{2}{|c|}{ Wateter } & \multicolumn{2}{|c|}{ 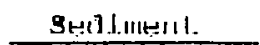 } & \multicolumn{2}{|c|}{ Veyelaliun } & \multicolumn{2}{|c|}{ Fisll } \\
\hline & $\begin{array}{c}90_{\mathrm{Sr}} \\
\end{array}$ & $\begin{array}{l}137 \mathrm{Cs} \\
(1,1)\end{array}$ & $\begin{array}{l}90 \mathrm{Sx} \\
(\mathrm{pCi}\end{array}$ & $\begin{array}{r}137 \mathrm{Cs} \\
\text { (wet) }\end{array}$ & $\begin{array}{l}90 \mathrm{Sr} \\
\text { (pCi }\end{array}$ & $\begin{array}{r}137 \mathrm{Cs} \\
- \text { sct) }\end{array}$ & $\begin{array}{l}90 \text { Sr } \\
\text { Flesh }\end{array}$ & $\begin{array}{l}137 \mathrm{Cs} \\
\text { Flooh }\end{array}$ \\
\hline & & & & & & & \multicolumn{2}{|c|}{$(p c i / K g-w e t)^{a}$} \\
\hline 1974 & 1.23 & 1.13 & - & 306 & - & 220 & - & $112-326$ \\
\hline 1975 & 1.74 & 4.46 & - & 525 & - & 1010 & - & 397 \\
\hline 1976 & 1.37 & 1.86 & - & 440 & - & 257 & - & 700 \\
\hline 1977 & 1.09 & 1.60 & - & 1656 & - & 1128 & $25-30$ & $772-3400$ \\
\hline $1978^{\circ}$ & 1.11 & 0.79 & - & 920 & - & 990 & $27-34$ & $536-1192$ \\
\hline 1979 & 0.58 & 0.87 & 19 & 188 & 86 & 585 & $40-60$ & $1036-1113$ \\
\hline
\end{tabular}

ariginal results given in dry weight; the results shown in Table are corrected to wet weight which facilitates estimation of concentration factors. 
these values have been consistent over many years. Well 非 which had previously showed larger than average concentrations in gross beta activity and in other pacameters such as coliform content, suggesting possible leakage in the well casing, has been discontinued.

\subsubsection{Ground Water Surveillance}

Samples of ground water were obtained from a network of shallow surveillance wells previously installed in the vicinity of several areas where a potential existed for the percolation of radioactivity from the surface downward into the saturated $z$ one of ground water. They include areas adjacent to on-site recharge basins, sand filter beds, downstream Peconic River, solid waste management area, former open dump, sanitary landfill, decontamination facility sump, and to the Meadow-Marsh Project area. The locations of most of these ground water surveillance wells are shown in Figure 9. The locations of the several wells installed at the landfill and solid waste management area are shown in Figure 10 .

For convenience in assessing the data, the wells have been divided into several groups. Yearly average gross alpha, gross beta, and tritium activity concentrations of the wells adjacent to the sand filter beds, and downstream on the Peconic River are summarized in Table 20. During the year, at least one sample from locations adjacent to the recharge basins and from locations immediately adjacent to the sand filter beds and the Peconic River were analyzed for ${ }^{90} \mathrm{Sr}$ and $137 \mathrm{Cs}$ (by gamma analysis) and are included in the table. Corresponding information for wells downstream (with reference to ground water movement) of the solid waste management area, the 1 andfill and former dump zones, and the decontamination facility sump (about $1 \mathrm{~km}$ east of the HFBR) is summarized in Table 21. Since the aquifer underlying Nassau and Suffolk Counties has been designated as a "Sole Source" (25), the EPA Drinking Water Standard is applicable (18). The data, therefore, is evaluated in terms of the EPA standard and not the RCG's, as was done in previous years.

In analyzing the data over the last six years (1974-1979), it has been observed that the spread of radioactivity in the ground water from Laboratory operations remained limited to within a few hundred meters of the identifiable foci. Above background activity concentrations of gross beta emitters, tritium and ${ }^{90} \mathrm{Sr}$ were fonnd nn-site adjacent to the sand filter beds and the Peconic River at small fractions of the Drinking Water Standards. In 1979, the activity concentrations were generally less than those noted in 1974 and 1975 , but were similar to that of 1976,1977 and 1978, indicating that radionuclides had not moved significantly since 1976. Wells XH and XZ which had shown a significant increase in gross beta activity in 1978 were reduced by more than $60 \%$ in 1979 . It must be noted, however, that these increases in 1978 were not accompanied by a similar increase in $90 \mathrm{Sr}$ activity. Wells $\mathrm{XK}, \mathrm{XL}, \mathrm{XN}, \mathrm{XX}$ and $\mathrm{XY}$ in proximity to the Peconic River did show slight increases in gross beta activity indicating the influence of the Peconic River. A similar influence was also seen in the gross alpha activity in wells $X M$ and $X N$. Adjacent to the Peconic River at the site boundary al1 activity concentrations (gross beta and tritium) were less than or equal to $4 \%$ of the Drinking Water Standards. Samples of well water collected from homes (Stations A, B, C and D - Figure 9) and well WS downstream 


\section{TARLE 19}

1979 BNI. POTABLE WATER AND COOL ING WATER WELLS

\begin{tabular}{|c|c|c|c|c|c|c|c|}
\hline & & $\begin{array}{l}\text { CROSSS } \\
\text { ALPHA } \\
\text { i PCI L I ) }\end{array}$ & ERFOR & $\begin{array}{l}\text { GROSS } \\
\text { BETA } \\
\text { (PCI/L) }\end{array}$ & ERROR & $\begin{array}{l}\text { TRITIUM } \\
\text { INCI/LI }\end{array}$ & ERROR \\
\hline $\mathrm{FB}$ & $\begin{array}{l}\text { MEAN } \\
\text { MINIMUM } \\
\text { MAXIMUM } \\
\text { NUMBER }\end{array}$ & $\begin{array}{l}0.31 \\
0.19 \\
0.58 \\
4\end{array}$ & $\begin{array}{l}0.13 \\
0.19 \\
0.35\end{array}$ & $\begin{array}{l}2.55 \\
2.14 \\
2.83 \\
4\end{array}$ & $\begin{array}{l}0.165 \\
0.90 \\
0.96\end{array}$ & $\begin{array}{l}0.26 \\
0.20 \\
0.31 \\
4\end{array}$ & $\begin{array}{l}0.13 \\
0.20 \\
0.31\end{array}$ \\
\hline FD & $\begin{array}{l}\text { MEAN } \\
\text { MINIMUM } \\
\text { MAXIMUM } \\
\text { MIJMBEK }\end{array}$ & $\begin{array}{l}0.30 \\
0.23 \\
0.38 \\
4\end{array}$ & $\begin{array}{l}0.14 \\
0.23 \\
0.28\end{array}$ & $\begin{array}{l}1.23 \\
0.87 \\
i .55 \\
4\end{array}$ & $\begin{array}{l}0.41 \\
0.85 \\
\dot{0.7 y}\end{array}$ & $\begin{array}{l}0.26 \\
0.20 \\
0.31 \\
4\end{array}$ & $\begin{array}{l}0.13 \\
0.20 \\
0.31\end{array}$ \\
\hline$r r$ & $\begin{array}{l}\text { PIE SNI } \\
\text { MINIMUM } \\
\text { MAXIMUM } \\
\text { NUMBER }\end{array}$ & $\begin{array}{l}0.24 \\
0.17 \\
0.32 \\
4\end{array}$ & $\begin{array}{l}0.12 \\
0.17 \\
0.32\end{array}$ & $\begin{array}{l}0.93 \\
0.75 \\
1.22 . \\
4 .\end{array}$ & $\begin{array}{l}\text { U.15 } \\
0.72 \\
1.22\end{array}$ & $\begin{array}{l}\text { U. } 010 \\
0.20 \\
6.31 \\
4\end{array}$ & $\begin{array}{l}0.13 \\
0.20 \\
0.31\end{array}$ \\
\hline$F F$ & $\begin{array}{l}\text { MEAN } \\
\text { MINIMUM } \\
\text { IAXIIIUII } \\
\text { NUMBER }\end{array}$ & $\begin{array}{l}0.35 \\
0.211 \\
0.717 \\
4\end{array}$ & $\begin{array}{l}0.15 \\
0.2: 1 \\
11 . .8 \%\end{array}$ & $\begin{array}{l}1.42 \\
1.19 \\
1.34 \\
4\end{array}$ & $\begin{array}{l}0.47 \\
1.19 \\
0.4 .4\end{array}$ & $\begin{array}{l}0.20 \\
0.20 \\
0.31 \\
4\end{array}$ & $\begin{array}{l}0.13 \\
0.200 \\
11.31\end{array}$ \\
\hline FO & $\begin{array}{l}\text { IEAN } \\
\text { MINIMUI" } \\
\text { MAXIMUM } \\
\text { NUMRFR }\end{array}$ & $\begin{array}{l}0.30 \\
0.17 \\
0.56 \\
3\end{array}$ & $\begin{array}{l}0.13 \\
0.17 \\
0.31\end{array}$ & $\begin{array}{l}0.9 \% \\
0.8 \% \\
1.20 \\
3\end{array}$ & $\begin{array}{l}0.54 \\
0.75 \\
\text { i. } 20\end{array}$ & $\begin{array}{l}0.27 \\
0.20 \\
0.31 \\
3\end{array}$ & $\begin{array}{l}0.16 \\
0.20 \\
0.31\end{array}$ \\
\hline $\mathbf{F H}$ & $\begin{array}{l}\text { MEAN } \\
\text { MINIMUM } \\
\text { MAYIMUM } \\
\text { NUMBER }\end{array}$ & $\begin{array}{l}0.21 \\
0.26 \\
0.20 \\
2\end{array}$ & $\begin{array}{l}0.19 \\
0.25 \\
0.20\end{array}$ & $\begin{array}{l}3.37 \\
2.40 \\
1.31 \\
2\end{array}$ & $\begin{array}{c}0.65 \\
0.84 \\
1.01 \\
.\end{array}$ & $\begin{array}{l}0.26 \\
0.21 \\
0.31 \\
0\end{array}$ & $\begin{array}{l}0.19 \\
0.21 \\
0.31\end{array}$ \\
\hline$F 1$ & $\begin{array}{l}\text { MEAN } \\
\text { MINIMUM } \\
\text { MAY!M!UM } \\
\text { NUMBER }\end{array}$ & $\begin{array}{l}0.28 \\
0.28 \\
0.28 \\
3\end{array}$ & $\begin{array}{l}0.16 \\
0.28 \\
0.28\end{array}$ & $\begin{array}{l}3.26 \\
1.97 \\
5.76 \\
3\end{array}$ & $\begin{array}{l}0.53 \\
0.90 \\
1.04\end{array}$ & $\begin{array}{l}0.24 \\
0.20 \\
0.31 \\
3\end{array}$ & $\begin{array}{l}0.14 \\
0.20 \\
0.31\end{array}$ \\
\hline F.J & $\begin{array}{l}\text { MEAN } \\
\text { PIINIMUM } \\
\text { MAX IIIU:1 } \\
\text { NUMGEP }\end{array}$ & $\begin{array}{l}0.55 \\
0.28 \\
0.95 \\
3\end{array}$ & $\begin{array}{l}0.19 \\
0.28 \\
8.41\end{array}$ & $\begin{array}{l}2.40 \\
2.27 \\
\dot{2} .4: \\
3\end{array}$ & $\begin{array}{l}0.31 \\
0.85 \\
U .86\end{array}$ & $\begin{array}{l}0.24 \\
0.20 \\
11.31 \\
3\end{array}$ & $\begin{array}{l}0 . ! 4 \\
0 . \bar{c} \\
0.31\end{array}$ \\
\hline $\mathrm{FI}_{-}$. & 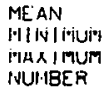 & $\begin{array}{l}0.24 \\
\text { U. } 16 \\
\text { U. 36 } \\
3\end{array}$ & $\begin{array}{l}0.14 \\
\text { J. Ib } \\
\text { U. } 3 .\end{array}$ & $\begin{array}{l}3.47 \\
3.106 \\
3.91\end{array}$ & $\begin{array}{l}0.54 \\
0.92 \\
0.91\end{array}$ & $\begin{array}{l}0.27 \\
0 . ? 1 \\
0.31 \\
3\end{array}$ & $\begin{array}{l}0.16 \\
0.21 \\
0.31\end{array}$ \\
\hline
\end{tabular}

\section{EPA-Drinking}

Water [18] and

Radiation Concentration

fuides [17]

3000 pC1/11ter for unidentifled nuclides in the absence of $y U_{5 r}, 22 y_{\mathrm{Ka}}$ or $\left.{ }^{12}\right)_{\mathrm{I}} ; 15 \mathrm{pci} / 11$ ter in the presence of $90 \mathrm{Sr}, 228 \mathrm{Ra}$ or ${ }^{12} 9_{\mathrm{I}}$.

20 nCi/liter - for Tritium $\left({ }^{3} \mathrm{H}\right)$

$\mathrm{nCi}=3.7 \times 10^{1} \mathrm{~Bq}$.

$\mathrm{pC} 1=3.7 \times 10^{-2} \mathrm{~Bq}$.

$F A: 1 \quad F E: 5 \quad F I: 102$

$F B: 2 \quad F F: 6 \quad F J: 103$

FC: 3 FG:7 FK:104

FD: 4 FH:101 FL:105 


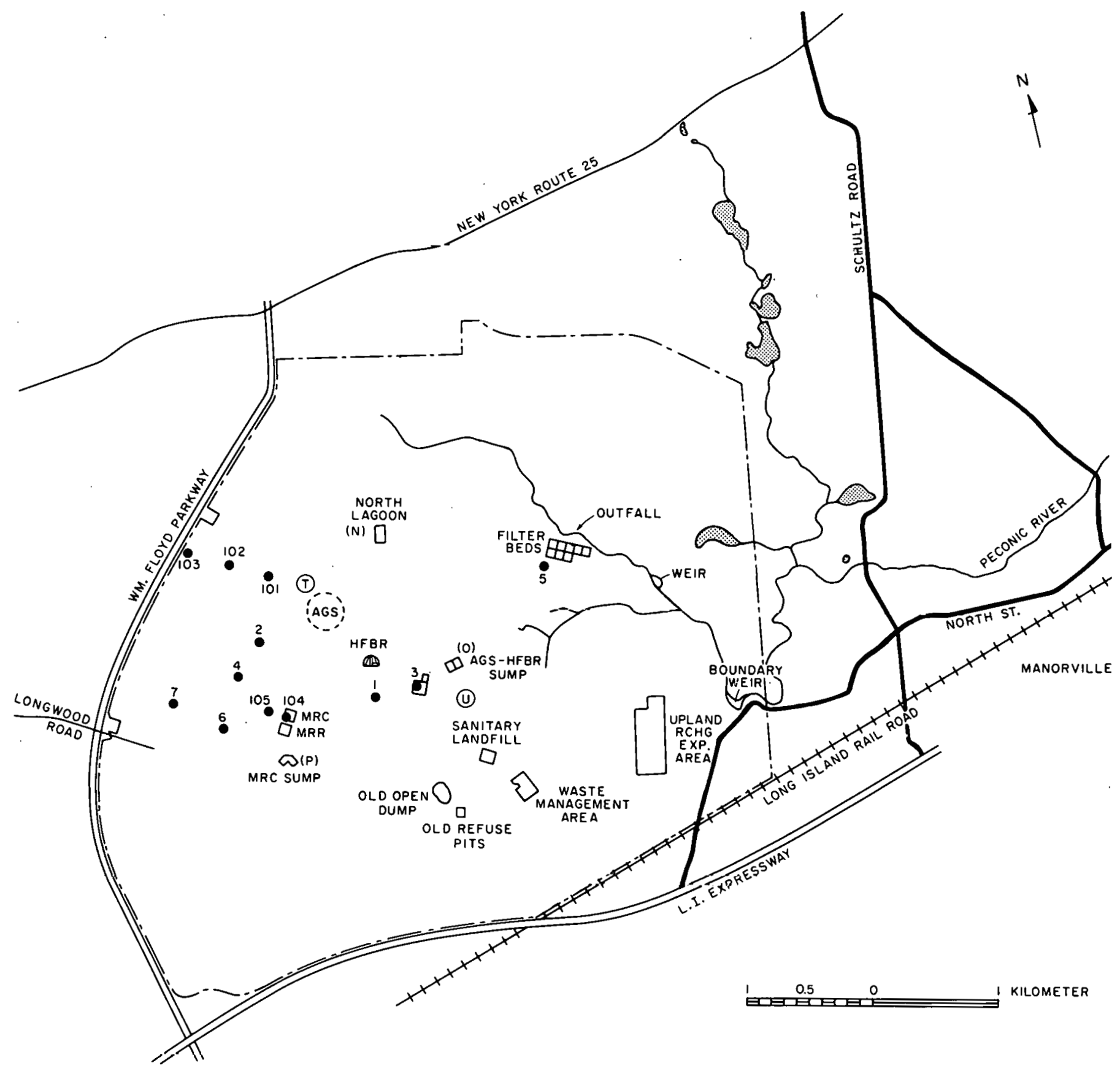

Figirte 8. On-sit.e Pntahlee and Supply Wells 


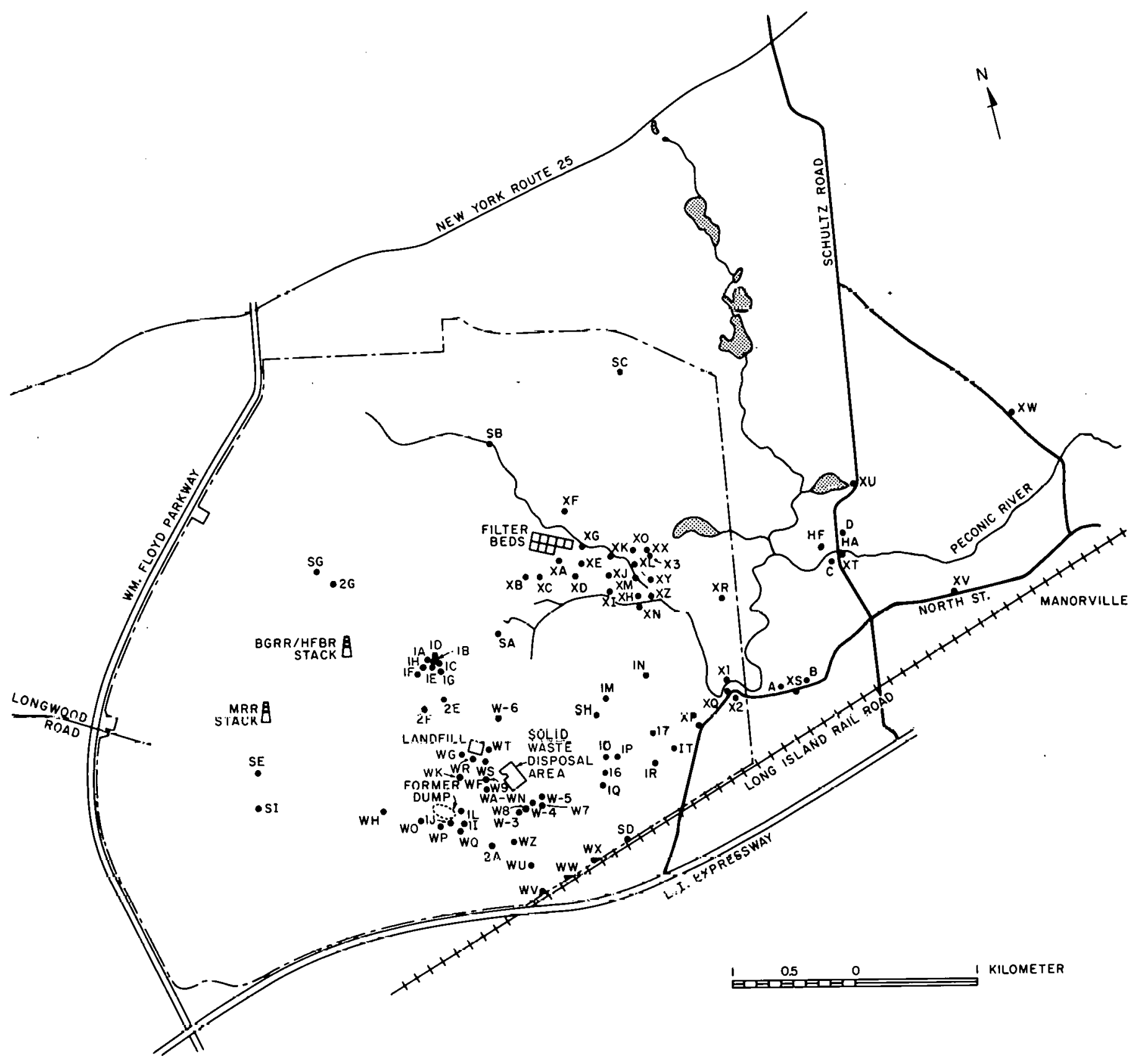

Figure 9. Location of Groundwater Surveillance Wells 


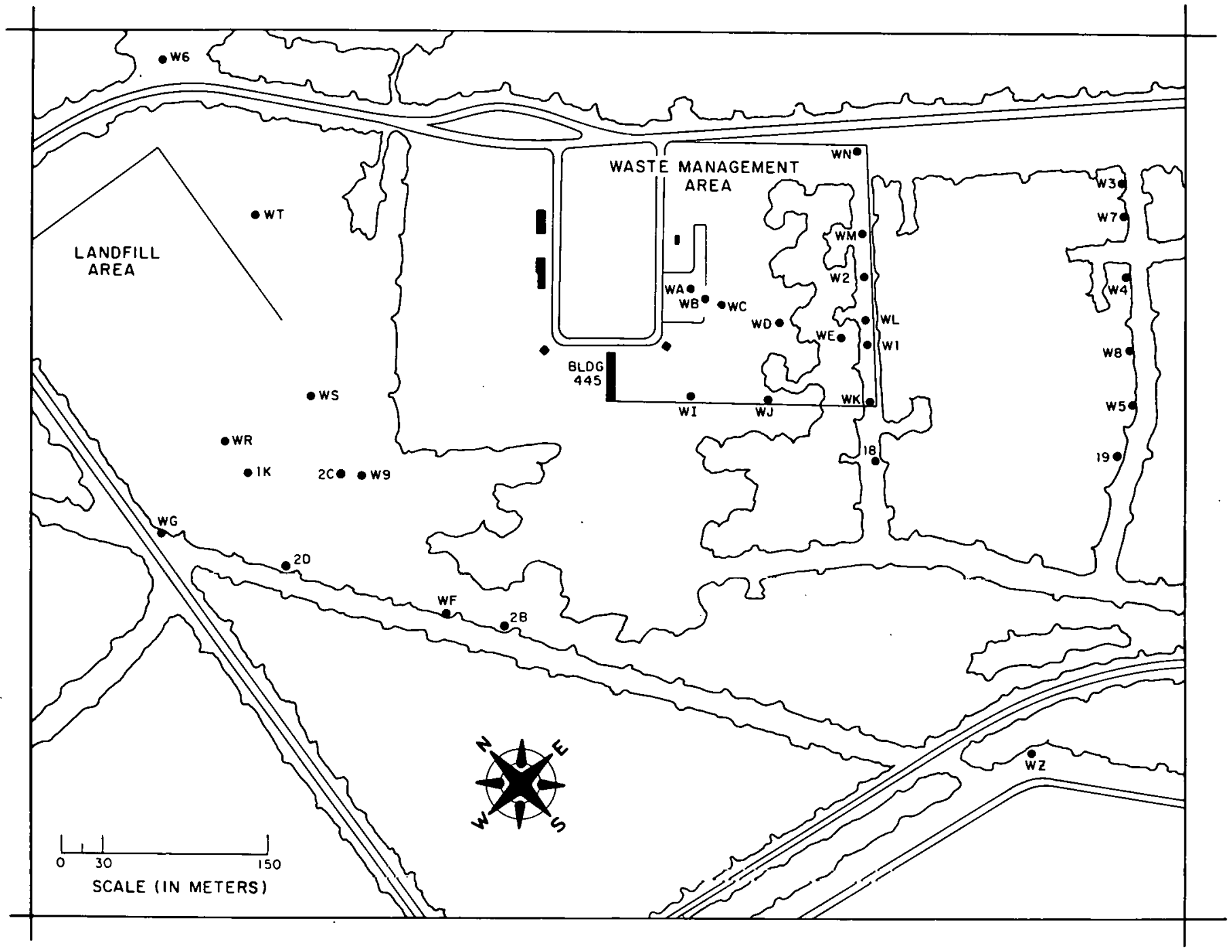

Figure 10. Landfill and Waste Management Area Surveillance Wells 
Table 20

1979 BNL SAND FILTER BED AND PECONIC RIVER AREA

\begin{tabular}{|c|c|c|c|c|c|c|c|c|c|}
\hline & & $\begin{array}{l}\text { GROSS } \\
\text { ALPHA } \\
\text { (PC) } 1 / L)\end{array}$ & ERROR & $\begin{array}{l}\text { GROSS } \\
\text { BEYA } \\
\text { [PCI/L) }\end{array}$ & ERROR & $\begin{array}{l}\text { TRI T I UM } \\
\text { (NCI/L) }\end{array}$ & ERROR & $\begin{array}{l}\text { SR90 } \\
\text { (PCI/L) }\end{array}$ & ERROR \\
\hline$X A$ & $\begin{array}{l}\text { MEAN } \\
\text { MINIMUM } \\
\text { MAXIMUM } \\
\text { NUIMBER }\end{array}$ & $\begin{array}{l}0.51 \\
0.20 \\
1.76 \\
8\end{array}$ & $\begin{array}{l}0.19 \\
0.20 \\
1.34\end{array}$ & $\begin{array}{r}11.41 \\
6.83 \\
15.45 \\
8\end{array}$ & $\begin{array}{l}0.52 \\
1.16 \\
1.48\end{array}$ & $\begin{array}{r}8.59 \\
0.50 \\
20.00 \\
8\end{array}$ & $\begin{array}{l}0.15 \\
0.29 \\
0.60\end{array}$ & $\begin{array}{l}1.89 \\
1.76 \\
1.98 \\
3\end{array}$ & $\begin{array}{l}0.09 \\
0.17 \\
0.19\end{array}$ \\
\hline XB & $\begin{array}{l}\text { MEAN } \\
\text { MINIMUM } \\
\text { MAXIMUM } \\
\text { NUMBER }\end{array}$ & $\begin{array}{l}0.32 \\
0.32 \\
0.32 \\
1\end{array}$ & $\begin{array}{l}0.32 \\
0.32 \\
0.32\end{array}$ & $\begin{array}{l}2.84 \\
2.84 \\
2.84 \\
1\end{array}$ & $\begin{array}{l}0.84 \\
0.84 \\
0.84\end{array}$ & $\begin{array}{l}0.22 \\
0.22 \\
0.22 \\
1\end{array}$ & $\begin{array}{l}0.22 \\
0.22 \\
0.22\end{array}$ & $\begin{array}{l}0.2 ! \\
0.21 \\
0.21 \\
1\end{array}$ & $\begin{array}{l}0.12 \\
0.12 \\
0.12\end{array}$ \\
\hline$x c$ & $\begin{array}{l}\text { MEAN } \\
\text { MIN!MUM } \\
\text { MAXIMUM } \\
\text { NUMBL.H }\end{array}$ & $\begin{array}{l}0.61 \\
0.61 \\
0.61 \\
1\end{array}$ & $\begin{array}{l}0.32 \\
0.32 \\
0.32\end{array}$ & $\begin{array}{l}5.78 \\
5.78 \\
5.78 \\
1\end{array}$ & $\begin{array}{l}0.98 \\
0.98 \\
0.98\end{array}$ & $\begin{array}{l}0.22 \\
0.22 \\
0.25 \\
j\end{array}$ & $\begin{array}{l}0.22 \\
0.22 \\
0.22\end{array}$ & $\begin{array}{l}1.91 \\
1.91 \\
1.91\end{array}$ & $\begin{array}{l}0.17 \\
0.17 \\
0.17\end{array}$ \\
\hline$x D$ & $\begin{array}{l}\text { MEAN } \\
\text { MINIMUM } \\
\text { MAX I MIJM } \\
\text { NI JMRER }\end{array}$ & 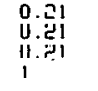 & 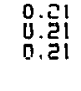 & $\mid \begin{array}{l}: 7 \\
1.7 \\
1.71\end{array}$ & $\begin{array}{l}8.76 \\
0.76 \\
0.76\end{array}$ & $\begin{array}{l}0.82 \\
0.22 \\
0.22 \\
1\end{array}$ & $\begin{array}{l}0.3 p \\
0.52 \\
0.52\end{array}$ & $\begin{array}{l}n .11 \\
0.11 \\
0.11 \\
1\end{array}$ & $\begin{array}{l}n \cap \cap A \\
n . \cap A \\
0.08\end{array}$ \\
\hline$X E$ & $\begin{array}{l}\text { MEAN } \\
\text { MINIMUM } \\
\text { MAXIMUM } \\
\text { NUHULIR }\end{array}$ & $\begin{array}{l}0.25 \\
0.25 \\
0.25 \\
1\end{array}$ & $\begin{array}{l}0.21 \\
0.21 \\
0.21\end{array}$ & $\begin{array}{l}1.77 \\
1.77 \\
1.77\end{array}$ & $\begin{array}{l}0.80 \\
0.80 \\
0.80\end{array}$ & $\begin{array}{l}0.20 \\
0.20 \\
0.20 \\
1\end{array}$ & $\begin{array}{l}0.20 \\
0.20 \\
0.20\end{array}$ & $\begin{array}{l}0.33 \\
0.33 \\
0.33 \\
1\end{array}$ & $\begin{array}{l}0.11 \\
0.11 \\
0.11\end{array}$ \\
\hline XF & $\begin{array}{l}\text { MEAN } \\
\text { MINIMUM } \\
\text { MAXIMUM } \\
\text { NUMBER }\end{array}$ & $\begin{array}{l}0.20 \\
0.20 \\
0.20 \\
1\end{array}$ & $\begin{array}{l}0.20 \\
0.20 \\
0.20\end{array}$ & $\begin{array}{l}1.13 \\
1.13 \\
1.13 \\
1\end{array}$ & $\begin{array}{l}1.13 \\
1.13 \\
1.13\end{array}$ & $\begin{array}{l}0.20 \\
0.20 \\
0.20 \\
1\end{array}$ & $\begin{array}{l}0.50 \\
0.50 \\
0.50\end{array}$ & \multicolumn{2}{|c|}{$<\mathrm{MDL}$} \\
\hline XG & MEAN & 0.40 & 0.10 & 3.83 & 0.35 & 0.24 & 0.08 & 0.89 & $0.0 \%$ \\
\hline$\rightarrow \quad--$ & $\begin{array}{l}\text { MINIMUM } \\
\text { MAXIMUM } \\
\text { NIIMRER }\end{array}$ & $\begin{array}{l}0.19 \\
0.89 \\
9\end{array}$ & $\begin{array}{l}0.19 \\
0.47\end{array}$ & $\begin{array}{l}2.2 b \\
7.30 \\
8\end{array}$ & $\begin{array}{l}0.86 \\
1.23\end{array}$ & $\begin{array}{l}0.22 \\
0.37 \\
0\end{array}$ & $\begin{array}{l}0.22 \\
0.37\end{array}$ & $\begin{array}{l}0.40 \\
1.67 \\
4\end{array}$ & $\begin{array}{l}0.10 \\
0.17\end{array}$ \\
\hline$x H$ & $\begin{array}{l}\text { MEAN } \\
\text { MINIPAUM } \\
\text { MAXIMUM } \\
\text { NUMBER }\end{array}$ & $\begin{array}{l}0.32 \\
0.32 \\
0.32 \\
1\end{array}$ & $\begin{array}{l}0.23 \\
0.23 \\
0.23\end{array}$ & $\begin{array}{l}0.98 \\
0.98 \\
0.98 \\
1\end{array}$ & $\begin{array}{l}0.75 \\
0.75 \\
0.75\end{array}$ & $\begin{array}{l}0.52 \\
0.25 \\
0.52 \\
1\end{array}$ & $\begin{array}{l}0.52 \\
0.25 \\
0.52\end{array}$ & & \\
\hline$x I$ & $\begin{array}{l}\text { MEAN } \\
\text { MINIMUM } \\
\text { MAXIMUM } \\
\text { NUMBER }\end{array}$ & $\begin{array}{l}0.47 \\
0.47 \\
0.47 \\
1\end{array}$ & $\begin{array}{l}0.28 \\
0.28 \\
0.28\end{array}$ & $\begin{array}{l}7.03 \\
7.03 \\
7.03 \\
1\end{array}$ & $\begin{array}{l}1.06 \\
1.06 \\
1.06\end{array}$ & $\begin{array}{l}0.23 \\
0.23 \\
0.23 \\
1\end{array}$ & $\begin{array}{l}0.23 \\
0.23 \\
0.23\end{array}$ & $\begin{array}{l}0.81 \\
0.17 \\
1.45 \\
2 .\end{array}$ & $\begin{array}{l}0.16 \\
0.04 \\
0.16\end{array}$ \\
\hline xu & $\begin{array}{l}\text { MEAN } \\
\text { MINIMUM } \\
\text { MAXIMUM } \\
\text { INUI+IBER }\end{array}$ & $\begin{array}{l}0.26 \\
0.26 \\
0.26 \\
1\end{array}$ & $\begin{array}{l}0.25 \\
0.22 \\
0.52\end{array}$ & $\begin{array}{l}3.75 \\
3.75 \\
3.75 \\
1\end{array}$ & $\begin{array}{l}0.90 \\
0.90 \\
0.90\end{array}$ & $\begin{array}{l}0.20 \\
0.20 \\
0.20 \\
1\end{array}$ & $\begin{array}{l}0.20 \\
0.20 \\
0.50\end{array}$ & $\begin{array}{l}.08 \\
1.08 \\
1.08\end{array}$ & $\begin{array}{l}0.14 \\
0.14 \\
0.14\end{array}$ \\
\hline XIC & 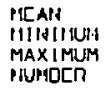 & $\begin{array}{l}0.05 \\
0.10 \\
1.49 \\
0\end{array}$ & $\begin{array}{l}0.15 \\
0.18 \\
0.93\end{array}$ & $\begin{array}{r}10.40 \\
7.80 \\
17.22 \\
9\end{array}$ & $\begin{array}{l}0.45 \\
1.25 \\
2.40\end{array}$ & $\begin{array}{l}1.45 \\
\text { U.31 } \\
3.18 \\
3\end{array}$ & $\begin{array}{l}\text { U. UY } \\
\text { U. } \\
0.30\end{array}$ & $\begin{array}{l}2.53 \\
2.14 \\
2.97 \\
5\end{array}$ & $\begin{array}{l}0.07 \\
0.19 \\
0.22\end{array}$ \\
\hline$X \mathrm{X}$ & $\begin{array}{l}\text { MEAN } \\
\text { MINIMUM } \\
\text { MAXIMUM } \\
\text { NUMBER }\end{array}$ & $\begin{array}{l}1.07 \\
0.68 \\
1.61 \\
6\end{array}$ & $\begin{array}{l}0.20 \\
0.38 \\
0.54\end{array}$ & $\begin{array}{c}24.28 \\
15.16 \\
31.26 \\
6\end{array}$ & $\begin{array}{l}0.73 \\
1.41 \\
2.01\end{array}$ & $\begin{array}{l}1.92 \\
1.03 \\
3.03 \\
6\end{array}$ & $\begin{array}{l}0.22 \\
0.39 \\
0.37\end{array}$ & $\begin{array}{l}5.88 \\
5.69 \\
7.19 \\
3\end{array}$ & $\begin{array}{l}0.16 \\
0.29 \\
0.31\end{array}$ \\
\hline YM & $\begin{array}{l}\text { ME. AM } \\
\text { MINIMUM } \\
\text { PLXIMUM }\end{array}$ & $\begin{array}{r}6.10 \\
1.45 \\
27.28 \\
0\end{array}$ & $\begin{array}{l}0.711 \\
0.5 .3 \\
5.54\end{array}$ & $\begin{array}{c}11.63 \\
11.37 \\
17.70 \\
0\end{array}$ & $\begin{array}{l}0.53 \\
1.31 \\
1.84\end{array}$ & $\begin{array}{l}5.09 \\
n .22 \\
5.45 \\
n\end{array}$ & $\begin{array}{l}0.17 \\
n .23 \\
0.35\end{array}$ & $\begin{array}{l}1.04 \\
1.51 \\
2.16 \\
2\end{array}$ & $\begin{array}{l}0.15 \\
0.15 \\
0.20\end{array}$ \\
\hline $\mathrm{XN}$ & $\begin{array}{l}\text { MEAN } \\
\text { MINIMUM } \\
\text { MAXIMUM } \\
\text { NUMBER }\end{array}$ & $\begin{array}{l}6.52 \\
6.52 \\
6.52 \\
1\end{array}$ & $\begin{array}{l}2.03 \\
2.03 \\
2.03\end{array}$ & $\begin{array}{c}20.93 \\
20.93 \\
1 \\
1\end{array}$ & $\begin{array}{l}4.75 \\
4.75 \\
4.75\end{array}$ & $\begin{array}{l}0.21 \\
0.21 \\
0.21 \\
1\end{array}$ & $\begin{array}{l}0.21 \\
0.21 \\
0.21\end{array}$ & $\begin{array}{l}0.14 \\
0 \\
0.14 \\
1 \\
1\end{array}$ & $\begin{array}{l}0.09 \\
\text { n no } \\
0.09\end{array}$ \\
\hline$\times 0$ & $\begin{array}{l}\text { MEAN } \\
\text { IIIINIIIUH } \\
\text { MAXIMUM } \\
\text { NI IMRFR }\end{array}$ & $\begin{array}{l}0.45 \\
0.10 \\
0.80 \\
5\end{array}$ & $\begin{array}{l}0.17 \\
0.16 \\
0.44\end{array}$ & $\begin{array}{l}6.40 \\
3.18 \\
9.10 \\
6\end{array}$ & $\begin{array}{l}0.52 \\
1.03 \\
1.23\end{array}$ & $\begin{array}{l}0.98 \\
\text { U. } \\
2.07 \\
5\end{array}$ & $\begin{array}{l}0.13 \\
0.23 \\
0.28\end{array}$ & $\begin{array}{l}1.18 \\
1.09 \\
1.29 \\
2\end{array}$ & $\begin{array}{l}0.10 \\
0.14 \\
0.15\end{array}$ \\
\hline$X R$ & $\begin{array}{l}\text { MEAN } \\
\text { MINIMUM } \\
\text { MAXIMUM } \\
\text { NUMBER }\end{array}$ & $\begin{array}{l}0.70 \\
0.70 \\
0.70 \\
1\end{array}$ & $\begin{array}{l}0.31 \\
0.31 \\
0.31\end{array}$ & $\begin{array}{l}5.23 \\
5.23 \\
5.23 \\
1\end{array}$ & $\begin{array}{l}0.98 \\
0.98 \\
0.98\end{array}$ & $\begin{array}{l}0.23 \\
0.23 \\
0.23 \\
1\end{array}$ & $\begin{array}{l}0.23 \\
0.23 \\
0.23\end{array}$ & $\begin{array}{l}1.80 \\
1.80 \\
1.80 \\
1\end{array}$ & $\begin{array}{l}0.17 \\
0.17 \\
0.17\end{array}$ \\
\hline XS & $\begin{array}{l}\text { MCAN } \\
\text { MINIMUM } \\
\text { MAXIMUM } \\
\text { NIIMRFR }\end{array}$ & $\begin{array}{l}2.32 \\
1.38 \\
3.31 \\
11\end{array}$ & $\begin{array}{l}0.22] \\
0.57 \\
0.89\end{array}$ & $\begin{array}{c}12.32 \\
9.34 \\
15.18 \\
10\end{array}$ & $\begin{array}{l}0.43 \\
1.29 \\
1.48\end{array}$ & $\begin{array}{l}0.25 \\
0.22 \\
0.32 \\
10\end{array}$ & $\begin{array}{l}0.08 \\
0.22 \\
0.32\end{array}$ & $\begin{array}{l}1.91 \\
1.92 \\
2.01 \\
4\end{array}$ & $\begin{array}{l}0.07 \\
0.19 \\
0.18\end{array}$ \\
\hline
\end{tabular}


Table 20 (continued)

1979 BNL SAND FILTER BED AND PECONIC RIVER AREA

\begin{tabular}{|c|c|c|c|c|c|c|c|c|c|}
\hline & & $\begin{array}{l}\text { GROSS } \\
\text { ALPHA } \\
\text { (PCI L ) }\end{array}$ & ERROR & $\begin{array}{l}\text { GROSS } \\
\text { BETA } \\
\text { (PCI/L) }\end{array}$ & ERROR & $\begin{array}{l}\text { TRITIUM } \\
\text { (NCI/L) }\end{array}$ & ERROR & $\begin{array}{l}\text { SR9G } \\
(\mathrm{PC} J / L)\end{array}$ & ERROR \\
\hline$X W$ & $\begin{array}{l}\text { MEAN } \\
\text { MINIMUM } \\
\text { MAXIMUM } \\
\text { NUMBER }\end{array}$ & $\begin{array}{l}0.19 \\
0.19 \\
0.19 \\
1\end{array}$ & $\begin{array}{l}0.19 \\
0.19 \\
0.19\end{array}$ & $\begin{array}{l}1.83 \\
1.83 \\
1.83 \\
1 .\end{array}$ & $\begin{array}{l}0.80 \\
0.80 \\
0.80\end{array}$ & $\begin{array}{l}1 \\
0.52 \\
0.52 \\
0.25\end{array}$ & $\begin{array}{l}0.25 \\
0.22 \\
0.25\end{array}$ & $\begin{array}{l}0.15 \\
0.15 \\
0.15 \\
1\end{array}$ & $\begin{array}{l}0.08 \\
0.08 \\
0.08\end{array}$ \\
\hline$x x$ & $\begin{array}{l}\text { MEAN } \\
\text { MINIMUM } \\
\text { MAXIMUM } \\
\text { NUMBER }\end{array}$ & $\begin{array}{l}0.90 \\
0.68 \\
1.18 \\
10\end{array}$ & $\begin{array}{l}0.13 \\
0.37 \\
0.50\end{array}$ & $\begin{array}{l}12.09 \\
7.80 \\
17.51 \\
10\end{array}$ & $\begin{array}{l}0.43 \\
1.12 \\
1.63\end{array}$ & $\begin{array}{l}2.19 \\
0.24 \\
11.20 \\
10\end{array}$ & $\begin{array}{l}0.09 \\
0.24 \\
0.40\end{array}$ & $\begin{array}{l}3.34 \\
2.12 \\
4.56 \\
4\end{array}$ & $\begin{array}{l}0.11 \\
0.18 \\
0.26\end{array}$ \\
\hline$X Y$ & $\begin{array}{l}\text { MEAN } \\
\text { MINIMUM } \\
\text { MAXIMUM } \\
\text { NUMBER }\end{array}$ & $\begin{array}{l}0.23 \\
0.23 \\
0.23 \\
1\end{array}$ & $\begin{array}{l}0.23 \\
0.23 \\
0.23\end{array}$ & $\begin{array}{l}11.81 \\
11.81 \\
11.81 \\
1\end{array}$ & $\begin{array}{l}1.28 \\
1.28 \\
1.28\end{array}$ & $\begin{array}{l}2.01 \\
2.01 \\
2.01 \\
1\end{array}$ & $\begin{array}{r}0.25 \\
0.25 \\
0.25\end{array}$ & $\begin{array}{l}2.68 \\
2.68 \\
2.68 \\
.\end{array}$ & $\begin{array}{l}0.19 \\
0.19 \\
0.19\end{array}$ \\
\hline$x z$ & $\begin{array}{l}\text { MEAN } \\
\text { MINIMUM } \\
\text { MAXIMUM } \\
\text { NUMBER }\end{array}$ & $\begin{array}{l}0.27 \\
0.27 \\
0.27 \\
1\end{array}$ & $\begin{array}{l}0.27 \\
\text { u. } \\
0.27\end{array}$ & $\begin{array}{l}3.60 \\
3.60 \\
3.60 \\
1\end{array}$ & $\begin{array}{l}0.88 \\
0.88 \\
0.88\end{array}$ & $\begin{array}{l}0.22 \\
0.22 \\
0.22 \\
1\end{array}$ & $\begin{array}{l}0.25 \\
0.22 \\
0.25\end{array}$ & $\begin{array}{l}0.55 \\
0.55 \\
0.55 \\
1\end{array}$ & $\begin{array}{l}0.11 \\
0.11 \\
0.11\end{array}$ \\
\hline$\times 1$ & $\begin{array}{l}\text { MEAN } \\
\text { MINIMUM } \\
\text { MAXIMUM } \\
\text { NUMBER }\end{array}$ & $\begin{array}{l}0.39 \\
0.39 \\
0.39 \\
1\end{array}$ & $\begin{array}{l}0.2^{4} \\
0.2^{4} \\
0.2^{4}\end{array}$ & $\begin{array}{l}2.51 \\
2.51 \\
2.51 \\
!\end{array}$ & $\begin{array}{l}0.84 \\
0.84 \\
0.84\end{array}$ & $\begin{array}{l}0.23 \\
0.23 \\
0.23 \\
1\end{array}$ & $\begin{array}{l}0.23 \\
0.23 \\
0.23\end{array}$ & & ' \\
\hline$x 2$ & $\begin{array}{l}\text { MEAN } \\
\text { MINIMUM } \\
\text { MAXIMUM } \\
\text { NUMBBER }\end{array}$ & $\begin{array}{l}0.17 \\
0.17 \\
0.17 \\
1\end{array}$ & $\begin{array}{l}0.17 \\
0.17 \\
0.17\end{array}$ & $\begin{array}{l}1.09 \\
1.09 \\
1.09\end{array}$ & $\begin{array}{l}1.09 \\
1.09 \\
1.09\end{array}$ & $\begin{array}{l}0.33 \\
0.33 \\
0.33 \\
1\end{array}$ & $\begin{array}{l}0.21 \\
0.21 \\
0.21\end{array}$ & \multicolumn{2}{|c|}{$<\mathrm{MDL}$} \\
\hline
\end{tabular}


TABLE 20 (Continued)

1979 BNL MISCELLANEOUS ON SITE

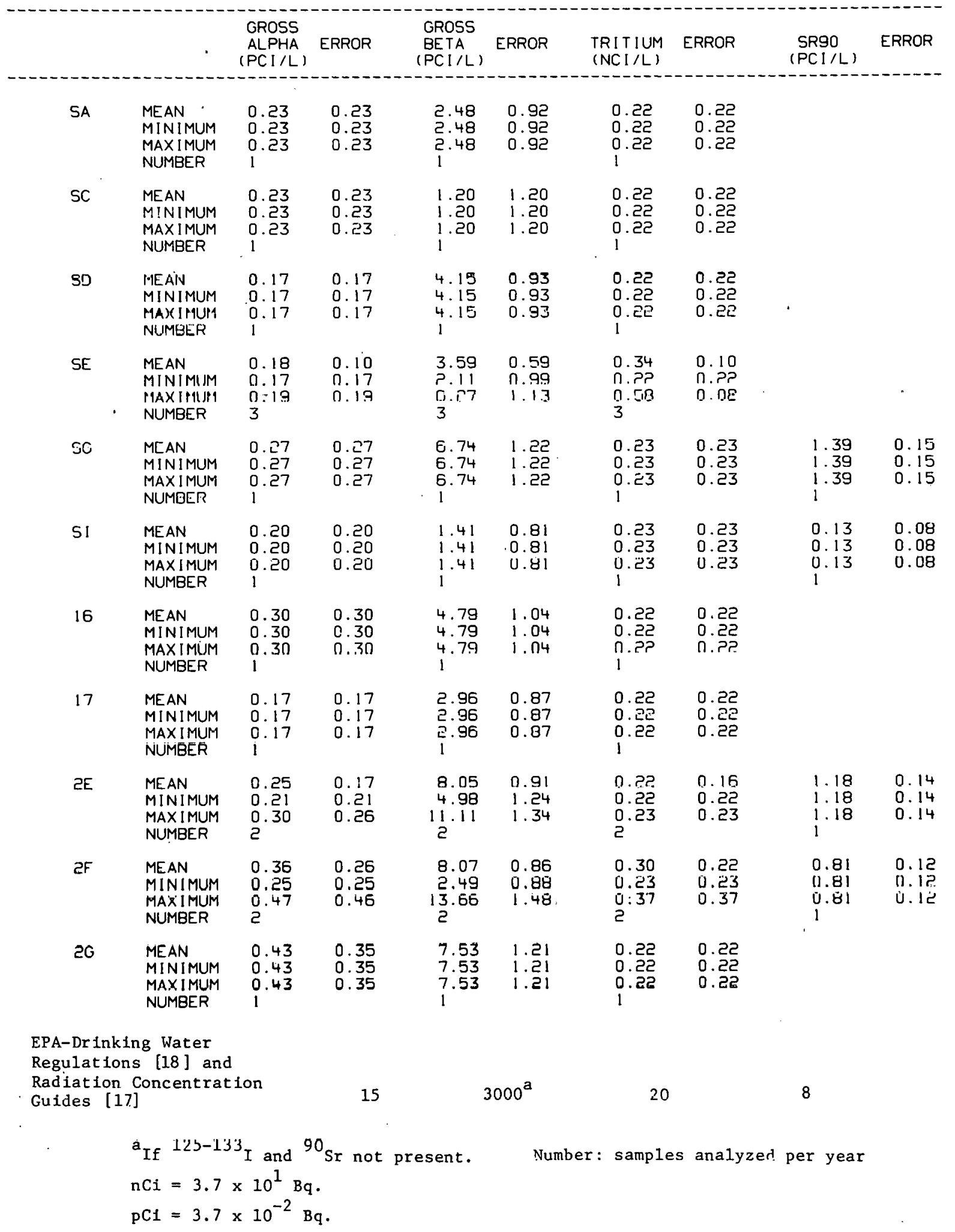


TABLE 21

1979 BNL WASTE MANAGEMENT AREA

PART

\begin{tabular}{|c|c|c|c|c|c|c|c|c|c|}
\hline & & $\begin{array}{l}\text { GROSS } \\
\text { ALPHA } \\
(P C I / L)\end{array}$ & ERROR & $\begin{array}{l}\text { GROSS } \\
\text { BETA } \\
\text { (PCI/L) }\end{array}$ & ERROR & $\begin{array}{l}\text { TRITIUH } \\
\text { (NCI/L) }\end{array}$ & ERROR & $\begin{array}{c}\text { SR9O } \\
(\mathrm{PCI} / \mathrm{L})\end{array}$ & ERROR \\
\hline WB & $\begin{array}{l}\text { MEAN } \\
\text { MINIMUM } \\
\text { MAXIMUM } \\
\text { NUMBER }\end{array}$ & $\begin{array}{l}0.72 \\
0.61 \\
0.81 \\
3\end{array}$ & $\begin{array}{l}0.21 \\
0.35 \\
0.37\end{array}$ & $\begin{array}{c}204.67 \\
42.54 \\
477.60 \\
3\end{array}$ & $\begin{array}{l}2.68 \\
2.17 \\
7.07\end{array}$ & $\begin{array}{l}10.00 \\
1.69 \\
20.00 \\
3\end{array}$ & $\begin{array}{l}0.77 \\
0 . .8 \\
0.60 \\
0.60\end{array}$ & $\begin{array}{c}40.97 \\
40.97 \\
40.97 \\
1\end{array}$ & $\begin{array}{l}0.72 \\
0.72 \\
0.72\end{array}$ \\
\hline WC & $\begin{array}{l}\text { MEAN } \\
\text { M!NIMUM } \\
\text { MAXIMUM } \\
\text { NUMBER }\end{array}$ & $\begin{array}{l}0.77 \\
0.77 \\
0.77 \\
1\end{array}$ & $\begin{array}{r}0.41 \\
0.41 \\
0.41 \\
?\end{array}$ & $\begin{array}{c}157.00 \\
157.00 \\
157.00 \\
1\end{array}$ & $\begin{array}{l}4.08 \\
4.08 \\
4.08\end{array}$ & $\begin{array}{l}21.90 \\
21.90 \\
21.90 \\
1\end{array}$ & $\begin{array}{l}0.60 \\
0.60 \\
0.60\end{array}$ & $\begin{array}{l}60.18 \\
60.18 \\
60.18 \\
1\end{array}$ & $\begin{array}{l}0.84 \\
0.84 \\
0.84\end{array}$ \\
\hline WD & $\begin{array}{l}\text { MEAN } \\
\text { MINIMUM } \\
\text { MAXIMUM } \\
\text { NUMBER }\end{array}$ & $\begin{array}{l}0.44 \\
0.25 \\
0.67 \\
5\end{array}$ & $\begin{array}{l}0.14 \\
0.25 \\
0.36\end{array}$ & $\begin{array}{l}27.07 \\
15.77 \\
39.07 \\
5\end{array}$ & $\begin{array}{l}0.80 \\
1.46 \\
2.09\end{array}$ & $\begin{array}{r}20.60 \\
2.75 \\
52.10 \\
5\end{array}$ & $\begin{array}{l}0.39 \\
0.30 \\
1.70\end{array}$ & $\begin{array}{c}9.47 \\
8.97 \\
10.01 \\
3\end{array}$ & $\begin{array}{l}0.36 \\
0.40 \\
0.28\end{array}$ \\
\hline WE & $\begin{array}{l}\text { MEAN } \\
\text { MINIMUM } \\
\text { MAXIMUUM } \\
\text { NUMBER }\end{array}$ & $\begin{array}{l}0.25 \\
0.25 \\
0.25 \\
1\end{array}$ & $\begin{array}{l}0.25 \\
0.25 \\
0.25\end{array}$ & $\begin{array}{l}15.36 \\
15.36 \\
15.36 \\
1\end{array}$ & $\begin{array}{l}1.36 \\
1.36 \\
1.36\end{array}$ & $\begin{array}{l}0.23 \\
0.23 \\
0.23 \\
i\end{array}$ & $\begin{array}{l}0.23 \\
0.23 \\
0.23\end{array}$ & $\begin{array}{l}5.83 \\
5.83 \\
5.83 \\
1\end{array}$ & $\begin{array}{l}0.27 \\
0.27 \\
0.27\end{array}$ \\
\hline WJ & $\begin{array}{l}\text { MEAN } \\
\text { MINIMUM } \\
\text { MAXIMUM } \\
\text { NUMBER }\end{array}$ & $\begin{array}{l}0.61 \\
0.61 \\
0.61 \\
1\end{array}$ & $\begin{array}{l}0.34 \\
0.34 \\
0.34\end{array}$ & $\begin{array}{l}9.01 \\
9.01 \\
9.01 \\
1\end{array}$ & $\begin{array}{l}1.16 \\
1.16 \\
1.16\end{array}$ & $\begin{array}{l}1.82 \\
1.82 \\
1.82 \\
1\end{array}$ & $\begin{array}{l}0.28 \\
0.28 \\
0.28\end{array}$ & $\begin{array}{l}0.84 \\
0.84 \\
0.84 \\
1\end{array}$ & $\begin{array}{l}0.13 \\
0.13 \\
0.13\end{array}$ \\
\hline WK & $\begin{array}{l}\text { MEAN } \\
\text { MINIMUM } \\
\text { MAXIMUM } \\
\text { NUMBER }\end{array}$ & $\begin{array}{l}1.5 ! \\
0.20 \\
2.23 \\
6\end{array}$ & $\begin{array}{l}0.51 \\
0.20 \\
0.61\end{array}$ & $\begin{array}{c}148.66 \\
79.49 \\
228.70 \\
6\end{array}$ & $\begin{array}{l}2.05 \\
2.87 \\
4.69\end{array}$ & $\begin{array}{l}5.39 \\
2.62 \\
9.05 \\
6\end{array}$ & $\begin{array}{l}0.23 \\
0.21 \\
0.43\end{array}$ & $\begin{array}{r}66.17 \\
34.70 \\
106.90 \\
.3\end{array}$ & $\begin{array}{l}0.49 \\
0.63 \\
1.20\end{array}$ \\
\hline WL & $\begin{array}{l}\text { MEAN } \\
\text { MINIMUM } \\
\text { MAXIMUM } \\
\text { NUMBER }\end{array}$ & $\begin{array}{l}0.88 \\
0.34 \\
1.80 \\
5\end{array}$ & $\begin{array}{l}0.18 \\
0.27 \\
0.55\end{array}$ & $\begin{array}{c}129.13 \\
69.28 \\
188.00 \\
5\end{array}$ & $\begin{array}{l}1.62 \\
2.70 \\
4.51\end{array}$ & $\begin{array}{l}3.11 \\
0.55 \\
5.52 \\
5\end{array}$ & $\begin{array}{l}0.26 \\
0.25 \\
1.15\end{array}$ & $\begin{array}{c}57.92 \\
35.69 \\
79.70 \\
3\end{array}$ & $\begin{array}{l}0.58 \\
0.71 \\
1.03\end{array}$ \\
\hline WM & $\begin{array}{l}\text { MEAN } \\
\text { MINIMUM } \\
\text { MAXIMUMM } \\
\text { NUMBER }\end{array}$ & $\begin{array}{l}0.61 \\
0.61 \\
0.61 \\
1\end{array}$ & $\begin{array}{l}0.38 \\
0.38 \\
0.38\end{array}$ & $\begin{array}{c}26.93 \\
26.93 \\
26.93 \\
1\end{array}$ & $\begin{array}{l}1.82 \\
1.82 \\
1.82\end{array}$ & $\begin{array}{l}4.11 \\
4.11 \\
4.11 \\
1\end{array}$ & $\begin{array}{l}0.34 \\
0.34 \\
0.34\end{array}$ & $\begin{array}{l}5.25 \\
2.52 \\
2.25 \\
1\end{array}$ & $\begin{array}{l}0.18 \\
0.18 \\
0.18\end{array}$ \\
\hline WN & $\begin{array}{l}\text { MEAN } \\
\text { MINIMIMM } \\
\text { MAXIMUM } \\
\text { NUMBER }\end{array}$ & $\begin{array}{l}0.23 \\
0.23 \\
0.23 \\
1\end{array}$ & $\begin{array}{l}0.23 \\
0.23 \\
0.23\end{array}$ & $\begin{array}{l}1.84 \\
1.84 \\
1.84 \\
1\end{array}$ & $\begin{array}{l}0.83 \\
0.8 .3 \\
0.83\end{array}$ & $\begin{array}{l}0.58 \\
0.58 \\
0.58 \\
1\end{array}$ & $\begin{array}{l}0.25 \\
0.75 \\
0.25\end{array}$ & $\begin{array}{l}0.11 \\
0.11 \\
0.11 \\
1\end{array}$ & $\begin{array}{l}0.08 \\
11.01 \% \\
0.08\end{array}$ \\
\hline WU & $\begin{array}{l}\text { MEAN } \\
\text { MINIMUM } \\
\text { MAXIMUM } \\
\text { NUMBER }\end{array}$ & $\begin{array}{l}0.27 \\
0.27 \\
0.27 \\
1\end{array}$ & $\begin{array}{l}0.27 \\
0.27 \\
0.27\end{array}$ & $\begin{array}{l}1.00 \\
1.00 \\
1.00 \\
1\end{array}$ & $\begin{array}{l}0.75 \\
0.75 \\
0.75\end{array}$ & $\begin{array}{l}0.20 \\
0.20 \\
0.20 \\
1\end{array}$ & $\begin{array}{l}0.20 \\
0.20 \\
0.20\end{array}$ & $\begin{array}{l}0.12 \\
0.12 \\
0.12 \\
1\end{array}$ & $\begin{array}{l}0.08 \\
0.08 \\
0.08\end{array}$ \\
\hline WV & $\begin{array}{l}\text { MEAN } \\
\text { MINIMUM } \\
\text { MAXIMUUM } \\
\text { NUMBER }\end{array}$ & $\begin{array}{l}0.27 \\
0.27 \\
0.27 \\
1\end{array}$ & $\begin{array}{l}0.27 \\
0.27 \\
0.27\end{array}$ & $\begin{array}{l}0.98 \\
0.98 \\
0.98 \\
1\end{array}$ & $\begin{array}{l}0.73 \\
0.73 \\
0.73\end{array}$ & $\begin{array}{l}0.20 \\
0.20 \\
0.20 \\
1 .\end{array}$ & $\begin{array}{l}0.20 \\
0.20 \\
0.20\end{array}$ & \multicolumn{2}{|c|}{ <MnT. } \\
\hline WW & $\begin{array}{l}\text { MEAN } \\
\text { MINIMUM } \\
\text { MAXIMUM } \\
\text { NIJMBER }\end{array}$ & $\begin{array}{l}0.13 \\
0.13 \\
0.13 \\
1\end{array}$ & $\begin{array}{l}0.13 \\
0.13 \\
0.13\end{array}$ & $\begin{array}{l}1.92 \\
1.72 \\
1.72 \\
1\end{array}$ & $\begin{array}{l}0.82 \\
0.82 \\
0.82\end{array}$ & $\begin{array}{l}0.20 \\
0.20 \\
0.20 \\
1\end{array}$ & $\begin{array}{l}0.20 \\
0.20 \\
0.20\end{array}$ & \multicolumn{2}{|c|}{$<$ MDL } \\
\hline
\end{tabular}


TABLE 21 (Continued)

1979 BNL WASTE MANAGEMENT AREA

PART 2

\begin{tabular}{|c|c|c|c|c|c|c|c|c|c|}
\hline & & $\begin{array}{l}\text { GROSS } \\
\text { ALPHA } \\
\text { (PCI/L ) }\end{array}$ & ERROR & $\begin{array}{l}\text { GROSS } \\
\text { BETA } \\
\text { (PCIIL) }\end{array}$ & ERROR & $\begin{array}{l}\text { TRITIUM } \\
\text { (NCIIL) }\end{array}$ & ERROR & $\begin{array}{c}\text { SR9O } \\
(P C \mid / L)\end{array}$ & ERROR \\
\hline$W X$ & $\begin{array}{l}\text { MEAN } \\
\text { MINIMUM } \\
\text { MAXIMUM } \\
\text { NUMBER }\end{array}$ & $\begin{array}{l}0.30 \\
0.30 \\
0.30 \\
1\end{array}$ & $\begin{array}{l}0.30 \\
0.30 \\
0.30\end{array}$ & $\begin{array}{l}1.14 \\
1.14 \\
1.14 \\
1\end{array}$ & $\begin{array}{l}0.76 \\
0.76 \\
0.76\end{array}$ & $\begin{array}{l}0.20 \\
0.20 \\
0.20 \\
1\end{array}$ & $\begin{array}{l}0.20 \\
0.20 \\
0.20\end{array}$ & & \\
\hline WZ & $\begin{array}{l}\text { MEAN } \\
\text { M!NIMUM } \\
\text { MAXIMUM } \\
\text { NUMBER }\end{array}$ & $\begin{array}{l}1.38 \\
1.38 \\
1.38\end{array}$ & $\begin{array}{l}1.38 \\
1.38 \\
1.38\end{array}$ & $\begin{array}{l}5.60 \\
5.60 \\
5.60 \\
1\end{array}$ & $\begin{array}{l}5.60 \\
5.60 \\
5.60\end{array}$ & $\begin{array}{l}0.20 \\
0.20 \\
0.20 \\
1\end{array}$ & $\begin{array}{l}0.200 \\
0.20 \\
0.20\end{array}$ & & \\
\hline WI & $\begin{array}{l}\text { MEAN } \\
\text { MINIMUM } \\
\text { MAXIMUM } \\
\text { NUMBER }\end{array}$ & $\begin{array}{l}\text { U. } 33 \\
0.20 \\
0.49 \\
5\end{array}$ & $\begin{array}{l}U .11 \\
0.20 \\
0.28\end{array}$ & $\begin{array}{l}18.24 \\
10.04 \\
27.03 \\
5\end{array}$ & $\begin{array}{l}0.66 \\
1.17 \\
1.74\end{array}$ & $\begin{array}{l}1.16 \\
0.36 \\
1.70 \\
5\end{array}$ & $\begin{array}{l}\text { U. } .^{44} \\
0.25 \\
1.10\end{array}$ & $\begin{array}{c}1.64 \\
4.30 \\
10.98 \\
2\end{array}$ & $\begin{array}{l}\text { U. }{ }^{\prime \prime} \\
0.25 \\
0.41\end{array}$ \\
\hline W3 & $\begin{array}{l}\text { MEAN } \\
\text { MINIMUM } \\
\text { MAXIMUM } \\
\text { NUMBER }\end{array}$ & $\begin{array}{l}0.15 \\
0.15 \\
0.15 \\
1\end{array}$ & $\begin{array}{l}0.15 \\
0.15 \\
0.15\end{array}$ & $\begin{array}{l}1.44 \\
1.44 \\
1.44 \\
1 .\end{array}$ & $\begin{array}{l}0.93 \\
0.93 \\
0.93\end{array}$ & $\begin{array}{l}0.31 \\
0.31 \\
0.31 \\
1\end{array}$ & $\begin{array}{l}0.31 \\
0.31 \\
0.31\end{array}$ & \multicolumn{2}{|c|}{$<\mathrm{MDL}$} \\
\hline W4 & $\begin{array}{l}\text { MEAN } \\
\text { MINIMUM } \\
\text { MAXIMUM } \\
\text { NUMBER }\end{array}$ & $\begin{array}{l}0.18 \\
0.18 \\
0.18 \\
1\end{array}$ & $\begin{array}{l}0.15 \\
0.15 \\
0.15\end{array}$ & $\begin{array}{l}3.78 \\
3.78 \\
3.78 \\
1\end{array}$ & $\begin{array}{l}1.13 \\
1.13 \\
1.13\end{array}$ & $\begin{array}{l}0.22 \\
0.22 \\
0.22 \\
1\end{array}$ & $\begin{array}{l}0.25 \\
0.25 \\
0.52\end{array}$ & $\begin{array}{l}0.13 \\
0.13 \\
0.13 \\
1\end{array}$ & $\begin{array}{l}0.09 \\
0.09 \\
0.09\end{array}$ \\
\hline WE & $\begin{array}{l}\text { MEAN } \\
\text { MINIMUM } \\
\text { MAXIMUM } \\
\text { NUMBER }\end{array}$ & $\begin{array}{l}0.36 \\
0.36 \\
0.36 \\
1\end{array}$ & $\begin{array}{l}0.21 \\
0.21 \\
0.21\end{array}$ & $\begin{array}{l}2.36 \\
2.36 \\
i .36 \\
1\end{array}$ & $\begin{array}{l}1.06 \\
1.06 \\
1.06\end{array}$ & $\begin{array}{l}0.22 \\
0.22 \\
0.22 \\
1\end{array}$ & $\begin{array}{l}0.25 \\
0.25 \\
0.52\end{array}$ & & \\
\hline W"I & $\begin{array}{l}\text { MFAN } \\
\text { MINIMUM } \\
\text { MAXIMUM } \\
\text { NUMBER }\end{array}$ & $\begin{array}{l}11.54 \\
0.24 \\
0.24 \\
1\end{array}$ & $\begin{array}{l}11.24 \\
0.24 \\
0.24\end{array}$ & $\begin{array}{l}1.6 \% \\
1.62 \\
1.62 \\
1\end{array}$ & $\begin{array}{l}0.84 \\
0.88 \\
0.88\end{array}$ & $\begin{array}{l}11.31 \\
0.31 \\
0.31 \\
1\end{array}$ & $\begin{array}{l}11.31 \\
0.31 \\
0.31\end{array}$ & \multicolumn{2}{|c|}{$<\mathrm{MDL}$} \\
\hline พ8 & $\begin{array}{l}\text { MEAN } \\
\text { MINIMUM } \\
\text { MAXIMUM } \\
\text { PUUMOCR }\end{array}$ & $\begin{array}{l}0.15 \\
0.15 \\
0.15 \\
1\end{array}$ & $\begin{array}{l}0.14 \\
0.14 \\
0.111\end{array}$ & $\begin{array}{l}2.84 \\
2.84 \\
2.84 \\
1\end{array}$ & $\begin{array}{l}1.10 \\
1.10 \\
1.10\end{array}$ & $\begin{array}{l}0.22 \\
0.22 \\
0.22 \\
1\end{array}$ & $\begin{array}{l}0.25 \\
0.52 \\
0.52\end{array}$ & & . \\
\hline 18 & $\begin{array}{l}\text { MEAN } \\
\text { MINIMUM } \\
\text { MAXIMUMM } \\
\text { NUMBER. }\end{array}$ & $\begin{array}{l}1.11 \\
1.11 \\
1.11 \\
1\end{array}$ & $\begin{array}{l}0.51 \\
0.51 \\
0.51\end{array}$ & $\begin{array}{l}3.95 \\
3.95 \\
3.95 \\
1\end{array}$ & $\begin{array}{l}0.99 \\
0.99 \\
0.99\end{array}$ & $\begin{array}{l}0.39 \\
0.39 \\
0.39 \\
1\end{array}$ & $\begin{array}{l}0.24 \\
0.24 \\
0.24\end{array}$ & $\begin{array}{l}0.12 \\
0.12 \\
0.12 \\
1\end{array}$ & $\begin{array}{l}0.08 \\
0.08 \\
0.08\end{array}$ \\
\hline 19 & $\begin{array}{l}\text { MEAN } \\
\text { MINIMUM } \\
\text { MAXIMUUM } \\
\text { NUMBER }\end{array}$ & $\begin{array}{l}0.43 \\
0.43 \\
0.43 \\
1\end{array}$ & $\begin{array}{l}0.28 \\
0.28 \\
0.28\end{array}$ & $\begin{array}{l}21.88 \\
21.88 \\
21.88 \\
1\end{array}$ & $\begin{array}{l}1.82 \\
1.62 \\
1.62\end{array}$ & $\begin{array}{l}0.23 \\
0.23 \\
0.23 \\
1\end{array}$ & $\begin{array}{l}0.23 \\
0.23 \\
0.23\end{array}$ & $\begin{array}{l}0.40 \\
0.40 \\
0.40 \\
1\end{array}$ & $\begin{array}{l}0.10 \\
0.10 \\
0.10\end{array}$ \\
\hline
\end{tabular}


TABLE 21 (Continued)

1979 BNL LAND F ILL AREA

\begin{tabular}{|c|c|c|c|c|c|c|c|c|c|}
\hline & & $\begin{array}{l}\text { CROSS } \\
\text { ALPHA. } \\
\text { 'PC, I'L, }\end{array}$ & ERROR & $\begin{array}{l}\text { GROSS } \\
\text { BETA } \\
\text { IH:I/ I }\end{array}$ & ERROR & $\begin{array}{l}\text { TR I T IUM } \\
\text { INEI I L I }\end{array}$ & ERROR & SR90 & ERROR \\
\hline$W F$ & $\begin{array}{l}\text { MEAN } \\
\text { MIN!MUM } \\
\text { MAXIMUM } \\
\text { NUMBER }\end{array}$ & $\begin{array}{l}0.25 \\
0.24 \\
0.25 \\
2\end{array}$ & $\begin{array}{l}0.18 \\
0.24 \\
0.25\end{array}$ & $\begin{array}{l}2.38 \\
2.14 \\
2.42 \\
2\end{array}$ & $\begin{array}{l}0.61 \\
0.82 \\
0.89\end{array}$ & $\begin{array}{l}1.51 \\
0.52 \\
7.70 \\
1\end{array}$ & $\begin{array}{l}1.11 \\
0.22 \\
2.20\end{array}$ & $\begin{array}{l}0.47 \\
0.47 \\
0.47 \\
1\end{array}$ & $\begin{array}{l}0.10 \\
0.10 \\
0.10\end{array}$ \\
\hline HG & $\begin{array}{l}\text { MEAN } \\
\text { P! NIMIMM } \\
\text { MAXIMUM } \\
\text { NUMBER }\end{array}$ & $\begin{array}{l}0.57 \\
0.23 \\
0 \\
0 \\
0\end{array}$ & $\begin{array}{l}0.17 \\
0.23 \\
0.24\end{array}$ & $\begin{array}{l}1.64 \\
0.97 \\
2.30 \\
2\end{array}$ & $\begin{array}{l}0.60 \\
0.76 \\
0.92\end{array}$ & $\begin{array}{l}0.55 \\
0.55 \\
0.0 .5 \\
0.0\end{array}$ & $\begin{array}{l}0.15 \\
0.22 \\
0.22\end{array}$ & $\begin{array}{l}0.17 \\
0.17 \\
0.17 \\
1\end{array}$ & $\begin{array}{l}0.10 \\
0.100 \\
0.10\end{array}$ \\
\hline$W R$ & $\begin{array}{l}\text { MEAN } \\
\text { MINIMUM } \\
\text { MAXIMUM } \\
\text { NUMBER }\end{array}$ & $\begin{array}{l}5.74 \\
5.74 \\
5.74 \\
1\end{array}$ & $\begin{array}{l}3.32 \\
3.32 \\
j .32\end{array}$ & $\begin{array}{c}86.68 \\
86.68 \\
86.68 \\
1\end{array}$ & $\begin{array}{l}8.19 \\
8.19 \\
8.19\end{array}$ & $\begin{array}{c}15.60 \\
15.60 \\
15.60 \\
1\end{array}$ & $\begin{array}{l}0.50 \\
0.50 \\
0.50\end{array}$ & $\begin{array}{l}5.29 \\
5.29 \\
5.29 \\
1\end{array}$ & $\begin{array}{l}0.29 \\
0.29 \\
0.29\end{array}$ \\
\hline WS & $\begin{array}{l}\text { MEAN } \\
\text { MINIMUM } \\
\text { MAXIMUM } \\
\text { NiMBEER }\end{array}$ & $\begin{array}{l}4.77 \\
2.27 \\
8.55 \\
4\end{array}$ & $\begin{array}{l}1.17 \\
1.53 \\
2.95\end{array}$ & $\begin{array}{l}54.91 \\
47.87 \\
65.28 \\
4\end{array}$ & $\begin{array}{l}3.29 \\
6.25 \\
6.71\end{array}$ & $\begin{array}{r}133.25 \\
12.20 \\
427.00 \\
4\end{array}$ & $\begin{array}{l}0.82 \\
0.50 \\
3.00\end{array}$ & $\begin{array}{l}2.73 \\
2.73 \\
2.73 \\
1\end{array}$ & $\begin{array}{l}0.21 \\
0.21 \\
0.21\end{array}$ \\
\hline WI & $\begin{array}{l}\text { MEAN } \\
\text { MINIMUM } \\
\text { MAXIMUM } \\
\text { NUMBER }\end{array}$ & $\begin{array}{c}5.44 \\
0.05 \\
10.03 \\
2\end{array}$ & $\begin{array}{l}1.47 \\
0.36 \\
2.42\end{array}$ & $\begin{array}{l}72.35 \\
57.15 \\
87.55 \\
2\end{array}$ & $\begin{array}{l}2.06 \\
2.44 \\
3.33\end{array}$ & $\begin{array}{l}0.29 \\
0.26 \\
0.32 \\
2\end{array}$ & $\begin{array}{l}0.15 \\
0.20 \\
0.32\end{array}$ & $\begin{array}{l}0.10 \\
0.10 \\
0.10 \\
1\end{array}$ & $\begin{array}{l}0.07 \\
0.07 \\
0.07\end{array}$ \\
\hline W9 & $\begin{array}{l}\text { MEAN } \\
\text { iTINIMUM } \\
\text { MAXIMUM } \\
\text { NUMBER }\end{array}$ & $\begin{array}{r}10.26 \\
5.19 \\
20.23 \\
4\end{array}$ & $\begin{array}{r}3.12 \\
2.88 \\
11.23\end{array}$ & $\begin{array}{l}7 c .19 \\
50.02 \\
80.06 \\
4\end{array}$ & $\begin{array}{l}3.84 \\
5.84 \\
8.72\end{array}$ & $\begin{array}{r}15.1,5 \\
0.22 \\
85.20 \\
4\end{array}$ & $\begin{array}{l}0.47 \\
0.22 \\
1.10\end{array}$ & $\begin{array}{l}5.34 \\
4.13 \\
8.54 \\
2\end{array}$ & $\begin{array}{l}0.21 \\
0.24 \\
0.34\end{array}$ \\
\hline$i k$ & $\begin{array}{l}\text { MEAN } \\
\text { MINIMUM } \\
\text { MAXIMUY } \\
\text { :HUMBCR }\end{array}$ & $\begin{array}{r}19.46 \\
7.19 \\
42.45 \\
3\end{array}$ & $\begin{array}{r}3.95 \\
3.6 ? \\
10.72\end{array}$ & $\begin{array}{r}78.95 \\
52.27 \\
116.80 \\
3\end{array}$ & $\begin{array}{l}4.62 \\
6.79 \\
9.34\end{array}$ & $\begin{array}{r}20.70 \\
4.69 \\
39.60 \\
3\end{array}$ & $\begin{array}{l}0.39 \\
0.33 \\
0.80\end{array}$ & $\begin{array}{l}5.10 \\
5.10 \\
5.10 \\
1\end{array}$ & $\begin{array}{l}0.33 \\
0.33 \\
0.33\end{array}$ \\
\hline $2 A$ & $\begin{array}{l}\text { MEAN } \\
\text { MINIMUM } \\
\text { MAXIMUM } \\
\text { NUMBER }\end{array}$ & $\begin{array}{l}0.22 \\
0.19 \\
0.25 \\
z\end{array}$ & $\begin{array}{l}0.16 \\
0.19 \\
0.25\end{array}$ & $\begin{array}{l}2.54 \\
1.45 \\
3.24 \\
2\end{array}$ & $\begin{array}{l}0.62 \\
0.80 \\
c .96\end{array}$ & $\begin{array}{l}0.26 \\
0.20 . \\
0.32 \\
2\end{array}$ & $\begin{array}{l}0.15 \\
0.20 \\
0.32\end{array}$ & $\begin{array}{l}0.44 \\
0.44 \\
0.44 \\
1\end{array}$ & $\begin{array}{l}0.10 \\
0.10 \\
0.10\end{array}$ \\
\hline $2 \theta$ & $\begin{array}{l}\text { MEAN } \\
\text { MINIMUM } \\
\text { MAXI:AUM } \\
\text { NUMBER }\end{array}$ & $\begin{array}{l}0.17 \\
0.12 \\
0.23 \\
2\end{array}$ & $\begin{array}{l}0.13 \\
0.12 \\
0.23\end{array}$ & $\begin{array}{l}7.99 \\
4.54 \\
1 ! .44 \\
2\end{array}$ & $\begin{array}{l}0.83 \\
1.09 \\
1.8 .6\end{array}$ & $\begin{array}{l}0.26 \\
0.20 \\
0.32 \\
?\end{array}$ & $\begin{array}{l}0.15 \\
0.20 \\
0.32\end{array}$ & \multicolumn{2}{|c|}{$<\mathrm{MNL}$} \\
\hline$\Sigma c$ & $\begin{array}{l}\text { ME AN } \\
\text { MINIMUM } \\
\text { MAXIMUM } \\
\text { NUMBER }\end{array}$ & $\begin{array}{l}4.34 \\
0.13 \\
7.913 \\
2\end{array}$ & $\begin{array}{l}1.96 \\
1.35 \\
3.95\end{array}$ & $\begin{array}{r}50.74 \\
1.11 \\
112.30 \\
?\end{array}$ & $\begin{array}{l}4.55 \\
1.12 \\
9.03\end{array}$ & $\begin{array}{l}49.20 \\
27.80 \\
68.80 \\
2\end{array}$ & $\begin{array}{l}0.64 \\
0.90 \\
0.90\end{array}$ & $\begin{array}{l}7.44 \\
7.44 \\
7.44 \\
1\end{array}$ & $\begin{array}{l}0.34 \\
0.34 \\
0.34\end{array}$ \\
\hline 20 & $\begin{array}{l}\text { MEAN } \\
\text { MINIMUM } \\
\text { MAXIMUM } \\
\text { SUMBER }\end{array}$ & $\begin{array}{l}18.48 \\
16.45 \\
20.51 \\
2\end{array}$ & $\begin{array}{l}5.14 \\
5.74 \\
8.52\end{array}$ & $\begin{array}{l}86.34 \\
53.78 \\
: 18.90 \\
2\end{array}$ & $\begin{array}{l}5.95 \\
7.03 \\
9.61\end{array}$ & $\begin{array}{r}32.45 \\
5.09 \\
58.80 \\
2\end{array}$ & $\begin{array}{l}0.67 \\
0.35 \\
1.30\end{array}$ & & \\
\hline
\end{tabular}


TARLE 21 (Continued)

1979 BNL 650 SUMP AREA AND FORMER DUMP AREA

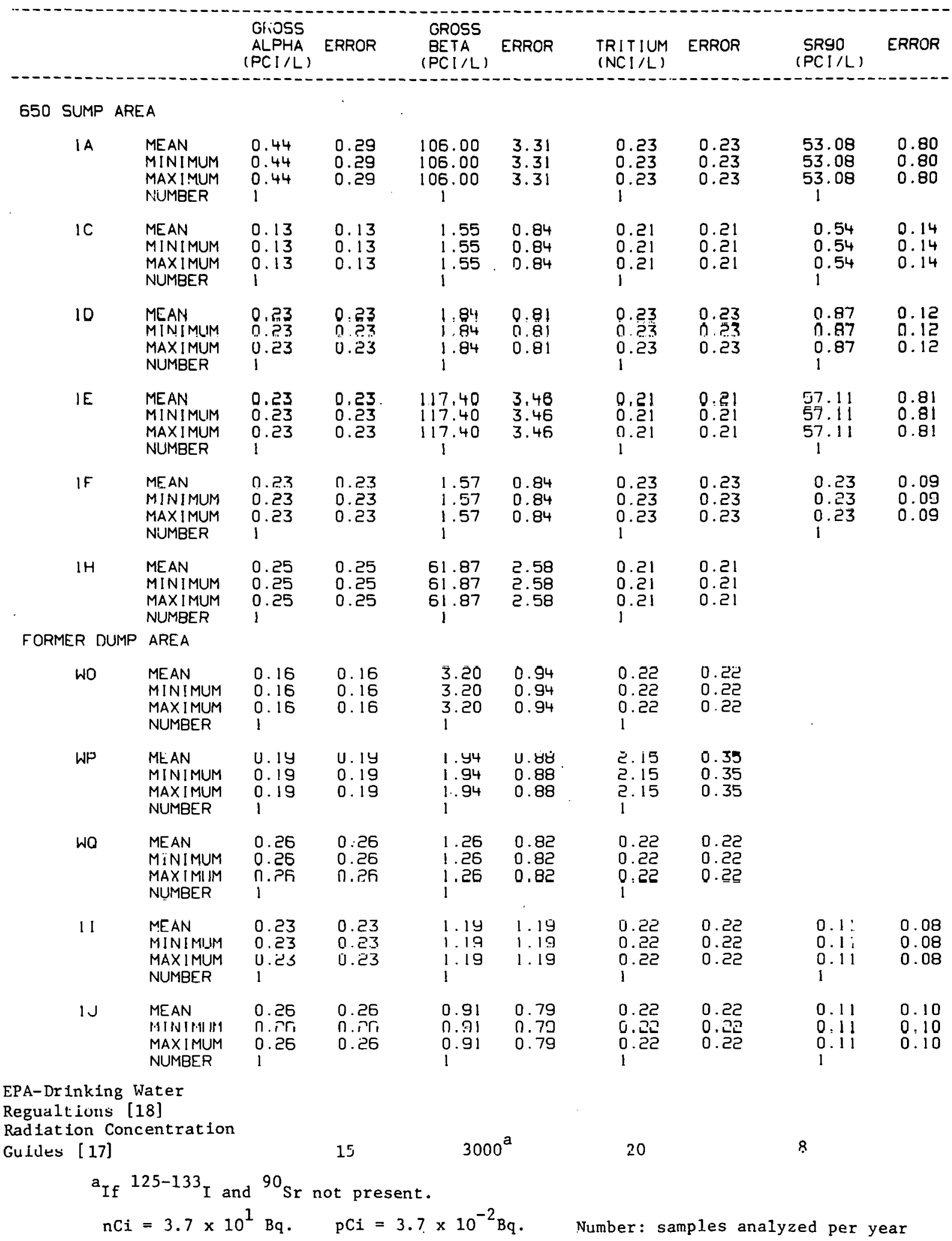


(with reference to ground water movement) of the Laboratory and the Peconic River, which had in 1978 indicated ${ }^{90} \mathrm{Sr}$ concentrations approaching one to two $\mathrm{pCi} \ell^{-1}$, showed a slight decrease in concentration. Whether the ${ }^{90} \mathrm{Sr}$ present in these wells result from Laboratory operations or not, the above values confirm that the EPA drinking water limit of $8 \mathrm{pCi} l^{-1}$ (18) has not been exceeded. In order to establish "background" levels of ${ }^{90} \mathrm{Sr}$ in local ground water remote from the possible influence of any BNL effluent, this Laboratory collaborated with the Suffolk County Department of Health Services (SCDHS) during July/August 1979 , in the collection and analysis of a number of samples from the county-wide network of test wells. Twenty three samples were collected from wells located from Babylon to Southhampton and having depths ranging from $8 \mathrm{~m}$ (shallow) to 86 $\mathrm{m}$ (deep). In addition, samples were collected from 20 private wells which are used as supply of drinking water in homes, schools and fire stations located primarily east of the Laboratory. All 43 samples were analyzed for ${ }^{90} \mathrm{Sr}$ by this Laboratory. The results, based on a minimum level of detection of $0.1 \mathrm{pCi} \ell^{-1}$ ${ }^{90} \mathrm{Sr}$, indicated that the largest concentration was found in four of the test wells and ranged from 1.1 to $2.75 \mathrm{\ell}^{-1} \mathrm{pCi}$ while the rest of the wells were below $0.5 \mathrm{pCi} \ell^{-1}$. When the ${ }^{90} \mathrm{Sr}$ concentrations were related to the depth of the water table, it was apparent that the largest concentrations were present in the shallow wells regardless of their proximity to the Laboratory. This observation casts doubt on the assumption made in the 1978 Environmental Monitoring Report that the presence of $90 \mathrm{Sr}$, in concentration of $1-2 \mathrm{pCi} \ell^{-1}$, in samples collected from shallow private wells located a short distance east of the Laboratory site and adjacent to the Peconic River was entirely the result of past releases by the Laboratory into the Peconic River. It now appears that most, if not all, of the ${ }^{90} \mathrm{Sr}$ concentrations found in these wells is most likely from fallout from past nuclear weapon tests. A review of the data on the levels of falloutrelated ${ }^{90} \mathrm{Sr}$ in precipitation shows that it was present in yearly average concentrations of more than $2 \mathrm{pCi} \ell^{-1}$ from 1953 to 1965 , with the exception of 1959. The maximum concentration measured locally was $26 \mathrm{pCi} \ell^{-1}$ in 1963 .

According to local and state radiological health authorities, the health significance of these small amounts of $90 \mathrm{Sr}$ is minimal. The EPA limit for $90 \mathrm{Sr}$ in a community water supply (applicable to the ground water in Suffolk as a "sole source" of drinking water $(25)$ ) is $8 \mathrm{pCi} \ell^{-1}(18)$. This would correspond to a yearly dose commitment of four millirems.

The current average concentration of past weapons testing related $90 \mathrm{Sr}$ in milk from a local dairy is about $14 \mathrm{pCi} \ell^{-1}$ while the average total dietary intake of ${ }^{90} \mathrm{Sr}$ in the New York area is about $8 \mathrm{pCi} \mathrm{d}^{-1}$. A yearly dose commitment of four millirems is with in the local fluctuations of external background radiation, which averages about 70 millirems per year on Long Island.

Compared with the values detected in 1974-1978, the gross beta, tritium and $90 \mathrm{Sr}$ activity concentrations for 1979 have, in most cases, decreased to $50 \%$ of the values in 1977 in several wells adjacent to the solid waste management area. The elevated ${ }^{90} \mathrm{Sr}$ activity concentrations, exceeding Drinking Water Standards, in we $11 \mathrm{~s} \mathrm{WK}, \mathrm{WL}, \mathrm{WB}$ and $\mathrm{WC}$ continue to reflect the inadvertent injection in 1960 of approximately $1 \mathrm{Ci}\left(3.7 \times 10^{10} \mathrm{~Bq}\right)$ of this nuclide into ground water at well WA. The concentrations in wells WK and WL, which had decreased by 20 to $30 \%$ of the 1977 levels, remained at the level seen in 1978, while well WB, 
which is adjacent to WA, has decreased by a factor of 2 , but we 11 WC has increased to 12 times the 1978 level. Other wells, such as WD, WE, WM, W1 and 19 have all shown decreases in gross beta and ${ }^{90} \mathrm{Sr}$ activity when compared to 1978 levels. Such fluctuations represent the complex interaction of ground water movement rates and distribution coefficients of the elements in the soil matrix. The gross beta and tritium activity concentrations, which had shown an increasing trend since 1974, decreased in several wells immediately adjacent to the landfill area and reflects movement and dilution through ground water. A further decrease in activity concentrations was apparent in wells adjacent to the former open dump when compared to the years 1974-1978. The gross beta and ${ }^{90} \mathrm{Sr}$ values in we $1 \mathrm{ls} 1 \mathrm{~A}$ and $1 \mathrm{H}$, monitoring the decontamination facility (Bldg. 650) sump, which had increased by a factor of two in 1978 showed significant reductions in activity in 1979 but well $1 \mathrm{E}$ has increased twofold without a similar increase in $90 \mathrm{Sr}$ concentration, while the other wells around this sump area have further decreased when compared to the concentrations during 1974-1978. In view of the new standards that are applicable to ground water systems, the gross beta and ${ }^{90} \mathrm{Sr}$ indeed exceed the limits. However, in the case of ${ }^{90} \mathrm{Sr}$, calculations based on ground water travel times, $90 \mathrm{Sr}$ distribution coefficient for ionexchange and distance to potential user (as drinking water) indicate travel time greater than two ${ }^{90} \mathrm{Sr}$ half-1ives (approximately 60 years) to reach areas where exposure can occur. Based on the existing levels in the above wells, the Laboratory does not foresee this inadvertent dumping of $90 \mathrm{Sr}$ in we 11 WA and the 650 sump area will cause the concentrations of $90_{\mathrm{Sr}}$ in wells off-site to exceed EPA drinking water limits. A study on $90 \mathrm{Sr}$ in groundwater discussed elsewhere in this section further substantiates the above observation.

Several water quality and purity parameters were evaluated for all ground water surveillance wells. The data for those wells nearby to on-site sumps, the sand filter beds, and downstream of the Peccnic River on- and off-site, are shown in Table 22. Similarly, the data for wells nearby to the solid waste management area, the landfill, the dump area and the $650 \mathrm{sump}$, are shown in Table 23. Table 24 presents similar data for potable and cooling water supply wells. This data is further compared with tap water, for a few of the parameters, in the same table. In all cases, the ground water guality parameters were within standard limits. Analyses for selected metals were conducted for a few wells immediately adjacent to the sand filter beds, to the Peconic River, to the waste management, landfill and former dump areas. These data are shown in Table 25 . Results of trace element analyses for potable and cooling water supply wells, and tap water are given in Table 26.

In genera1, tho data were comparable to that obseived luring 1974-1978. With the exception of $\mathrm{pH}$, all analyzed water quality parameters were within New York State Water Quality Standards (13). The somewhat lower pH levels appear to reflect natural ambient levels, since higher $\mathrm{pH}$ levels were present in the input to and output from the sewage treatment plant (see Table 10). Concentrations of $\mathrm{Fe}, \mathrm{Zn}$ and $\mathrm{Pb}$ in excess of water quality standards were found in some of the wells immediately adjacent to the sand filter beds, the Peconic River, 1 andfill areas, and the 650 sump area. It is not clear to what extent they may be an artifact produced by the sampling well casings, or reflect the leaching of accumulations of these metals from past Laboratory releases. A program to compare effects of well casings has been instituted in 1980. Tracing the levels of these 
TABLE 22

1979 BNL ENVIRONMENTAL MONITORING SAND FILTER BEOS AND PECONIC RIVER AREA WELLS WATER QUALITY AND PURITY

\begin{tabular}{|c|c|c|c|c|c|c|c|c|c|c|c|}
\hline LOCATION & $\checkmark$ SAMPLE & TEMPERATURE & PH & $\begin{array}{l}\text { DISSOLVED } \\
\text { OXYGEN } \\
\text { (PPM) }\end{array}$ & $\begin{array}{l}\text { CHLORIDES } \\
\text { (PPM) }\end{array}$ & $\begin{array}{l}\text { NI TRATE } \\
\text { Ni TROGEN } \\
\text { (PPM) }\end{array}$ & $\begin{array}{l}\text { TOTAL } \\
\text { PHOSPHOROUS } \\
\text { (PPM) }\end{array}$ & $\begin{array}{l}\text { DISSOLVED } \\
\text { SOLIDS } \\
\text { (PPM) }\end{array}$ & $\begin{array}{l}\text { CONDUC- } \\
\text { T!VITY } \\
\text { MICROMHO/CM }\end{array}$ & $\begin{array}{c}\text { COL IFORM } \\
\text { FECAL } \\
(/ 100 \mathrm{ML})\end{array}$ & $\begin{array}{c}\text { COL IFORM } \\
\text { TOTAL } \\
(/ 100 \mathrm{ML})\end{array}$ \\
\hline$X A$ & $\begin{array}{l}\text { MEAN } \\
\text { MINIMUM } \\
\text { MAXIMUM } \\
\text { NUMBER }\end{array}$ & $\begin{array}{r}17 \\
14 \\
22 \\
8\end{array}$ & $\begin{array}{l}5.5 \\
5.2 \\
5.8 \\
8\end{array}$ & $\begin{array}{l}4.4 \\
2.0 \\
7.2 \\
8\end{array}$ & $\begin{array}{c}27.0 \\
22.3 \\
32.8 \\
8\end{array}$ & $\begin{array}{l}6.41 \\
3.33 \\
11.47 \\
8\end{array}$ & $\begin{array}{l}0.03 \\
0.01 \\
0.12 \\
8\end{array}$ & $\begin{array}{r}131 \\
117 \\
142 \\
7\end{array}$ & $\begin{array}{r}175 \\
154 \\
198 \\
8\end{array}$ & $\begin{array}{l}0 \\
0 \\
0 \\
8\end{array}$ & $\begin{array}{l}0 \\
0 \\
0 \\
8\end{array}$ \\
\hline$X B$ & $\begin{array}{l}\text { MEAN } \\
\text { MINIMUM } \\
\text { MAXIMUM } \\
\text { NUMBER }\end{array}$ & $\begin{array}{r}10 \\
10 \\
10 \\
1\end{array}$ & $\begin{array}{l}5.3 \\
5.3 \\
5.3 \\
1\end{array}$ & $\begin{array}{l}7.8 \\
7.8 \\
7.8 \\
1\end{array}$ & $\begin{array}{l}31.5 \\
31.5 \\
3 ! .5 \\
1\end{array}$ & $\begin{array}{l}0.03 \\
0.03 \\
0.03 \\
1\end{array}$ & $\begin{array}{l}0.01 \\
0.01 \\
0.01 \\
1\end{array}$ & $\begin{array}{r}157 \\
157 \\
157 \\
1\end{array}$ & $\begin{array}{l}160 \\
160 \\
160 \\
1\end{array}$ & $\begin{array}{l}0 \\
0 \\
0 \\
1\end{array}$ & $\begin{array}{l}0 \\
0 \\
0 \\
1\end{array}$ \\
\hline$x C$ & $\begin{array}{l}\text { MEAN } \\
\text { M!NIMUM } \\
\text { MAXIMUM } \\
\text { NUMBER }\end{array}$ & $\begin{array}{r}10 \\
10 \\
10 \\
1\end{array}$ & $\begin{array}{l}5.3 \\
6.3 \\
6.3 \\
1\end{array}$ & $\begin{array}{l}3.8 \\
3.8 \\
3.8 \\
1\end{array}$ & $\begin{array}{l}13.5 \\
13.5 \\
13.5 \\
1\end{array}$ & $\begin{array}{l}0.03 \\
0.03 \\
0.03 \\
1\end{array}$ & $\begin{array}{l}0.01 \\
0.01 \\
0.01 \\
1\end{array}$ & $\begin{array}{r}65 \\
65 \\
65 \\
1\end{array}$ & $\begin{array}{l}87 \\
87 \\
87 \\
1\end{array}$ & $\begin{array}{l}0 \\
0 \\
0 \\
1\end{array}$ & $\begin{array}{l}0 \\
0 \\
0 \\
1\end{array}$ \\
\hline XD & $\begin{array}{l}\text { MEAN } \\
\text { MINIMUM } \\
\text { MAXIMUM } \\
\text { NUMBER }\end{array}$ & $\begin{array}{r}10 \\
10 \\
10 \\
1\end{array}$ & $\begin{array}{l}5.6 \\
5.6 \\
5.6 \\
1\end{array}$ & $\begin{array}{l}2.6 \\
2.6 \\
2.6 \\
1\end{array}$ & $\begin{array}{l}11.5 \\
11.5 \\
11.5 \\
1\end{array}$ & $\begin{array}{l}0.06 \\
0.06 \\
0.06 \\
1\end{array}$ & $\begin{array}{l}0.01 \\
0.01 \\
0.01 \\
1\end{array}$ & $\begin{array}{c}48 \\
48 \\
48 \\
1\end{array}$ & $\begin{array}{c}64 \\
64 \\
64 \\
1\end{array}$ & $\begin{array}{l}0 \\
0 \\
0 \\
1\end{array}$ & $\begin{array}{l}0 \\
0 \\
0 \\
1\end{array}$ \\
\hline$X E$ & $\begin{array}{l}\text { MEAN } \\
\text { MINIMUM } \\
\text { MAXIMIJM } \\
\text { NUMBER }\end{array}$ & $\begin{array}{r}12 \\
12 \\
12 \\
1\end{array}$ & $\begin{array}{l}5.3 \\
5.3 \\
5.3 \\
1\end{array}$ & $\begin{array}{l}6.6 \\
6.6 \\
6.6 \\
1\end{array}$ & $\begin{array}{l}8.2 \\
8.2 \\
8.2 \\
1\end{array}$ & $\begin{array}{l}0.54 \\
0.54 \\
0.54 \\
1\end{array}$ & $\begin{array}{l}0.03 \\
0.03 \\
0.03 \\
1\end{array}$ & $\begin{array}{l}1 \\
1 \\
1 \\
1\end{array}$ & $\begin{array}{r}63 \\
63 \\
63 \\
1\end{array}$ & $\begin{array}{l}0 \\
0 \\
0 \\
1\end{array}$ & $\begin{array}{l}0 \\
0 \\
0 \\
1\end{array}$ \\
\hline XF & $\begin{array}{l}\text { MEAN } \\
\text { MINIMUM } \\
\text { MAXIMUM } \\
\text { NUMBER }\end{array}$ & $\begin{array}{l}11 \\
11 \\
11 \\
1\end{array}$ & $\begin{array}{l}5.4 \\
5.4 \\
5.4 \\
1\end{array}$ & $\begin{array}{l}4.2 \\
4.2 \\
4.2 \\
1\end{array}$ & $\begin{array}{l}11.7 \\
11.7 \\
11.7\end{array}$ & $\begin{array}{l}0.02 \\
0.02 \\
0.02 \\
1\end{array}$ & $\begin{array}{l}0.01 \\
0.01 \\
0.01 \\
1\end{array}$ & $\begin{array}{c}54 \\
54 \\
54 \\
1\end{array}$ & $\begin{array}{c}72 \\
72 \\
72 \\
1\end{array}$ & $\begin{array}{l}0 \\
0 \\
0 \\
1\end{array}$ & $\begin{array}{l}0 \\
0 \\
0 \\
1\end{array}$ \\
\hline$X G$ & $\begin{array}{l}\text { MEAN } \\
\text { MINIMUM } \\
\text { MAXIMUM } \\
\text { NUMBER }\end{array}$ & $\begin{array}{r}13 \\
9 \\
16 \\
9\end{array}$ & $\begin{array}{l}5.5 \\
5.1 \\
6.0 \\
9\end{array}$ & $\begin{array}{l}7.5 \\
5.4 \\
8.6 \\
9\end{array}$ & $\begin{array}{r}7.5 \\
4.0 \\
11.1 \\
9\end{array}$ & $\begin{array}{l}0.14 \\
0.01 \\
0.40 \\
9\end{array}$ & $\begin{array}{l}0.04 \\
0.01 \\
0.09 \\
9\end{array}$ & $\begin{array}{r}72 \\
58 \\
100 \\
9\end{array}$ & $\begin{array}{r}72 \\
58 \\
91 \\
9\end{array}$ & $\begin{array}{l}0 \\
0 \\
0 \\
6\end{array}$ & $\begin{array}{l}0 \\
0 \\
0 \\
6\end{array}$ \\
\hline $\mathbf{X H}$ & $\begin{array}{l}\text { MEAN } \\
\text { MINIMUM } \\
\text { MAXIMUM } \\
\text { NUMBER }\end{array}$ & $\begin{array}{l}11 \\
11 \\
11 \\
1\end{array}$ & $\begin{array}{l}5.1 \\
5.1 \\
5.1 \\
1\end{array}$ & $\begin{array}{l}3.0 \\
3.0 \\
3.0 \\
1\end{array}$ & $\begin{array}{l}9.2 \\
9.2 \\
9.2 \\
1\end{array}$ & $\begin{array}{l}0.04 \\
0.04 \\
0.04 \\
1\end{array}$ & $\begin{array}{l}0.01 \\
0.01 \\
0.01 \\
1\end{array}$ & $\begin{array}{l}69 \\
69 \\
69 \\
1\end{array}$ & $\begin{array}{r}53 \\
53 \\
53 \\
1\end{array}$ & $\begin{array}{l}0 \\
0 \\
0 \\
1\end{array}$ & $\begin{array}{l}0 \\
0 \\
0 \\
1\end{array}$ \\
\hline$x \mid$ & $\begin{array}{l}\text { MEAN } \\
\text { MINIMUM } \\
\text { MAXIMUM } \\
\text { NUMBER }\end{array}$ & $\begin{array}{l}9 \\
9 \\
9 \\
1\end{array}$ & $\begin{array}{l}5.6 \\
5.6 \\
5.6 \\
1\end{array}$ & $\begin{array}{l}6.6 \\
6.6 \\
6.6 \\
1\end{array}$ & $\begin{array}{l}13.5 \\
13.5 \\
13.5 \\
1\end{array}$ & $\begin{array}{l}0.03 \\
0.03 \\
0.03 \\
1\end{array}$ & $\begin{array}{l}0.01 \\
0.01 \\
0.01 \\
1\end{array}$ & $\begin{array}{r}35 \\
35 \\
35 \\
1\end{array}$ & $\begin{array}{r}54 \\
54 \\
54 \\
1\end{array}$ & $\begin{array}{l}0 \\
0 \\
0 \\
1\end{array}$ & $\begin{array}{l}0 \\
0 \\
0 \\
1\end{array}$ \\
\hline$x J$ & $\begin{array}{l}\text { MEAN } \\
\text { MINIMUM } \\
\text { MAXIMUM } \\
\text { NUMBER }\end{array}$ & $\begin{array}{ll}1 & 1 \\
11 \\
11 \\
11\end{array}$ & $\begin{array}{l}5.1 \\
5.1 \\
5.1 \\
1\end{array}$ & $\begin{array}{l}1.6 \\
1.6 \\
1.6\end{array}$ & $\begin{array}{l}6.1 \\
6.1 \\
6.1 \\
1\end{array}$ & $\begin{array}{l}0.02 \\
0.02 \\
0.02 \\
1\end{array}$ & $\begin{array}{l}0.03 \\
0.03 \\
0.03 \\
1\end{array}$ & $\begin{array}{r}40 \\
40 \\
40 \\
1\end{array}$ & $\begin{array}{l}47 \\
47 \\
47 \\
1\end{array}$ & $\begin{array}{l}0 \\
0 \\
0 \\
1\end{array}$ & $\begin{array}{l}0 \\
0 \\
0 \\
1\end{array}$ \\
\hline XK & $\begin{array}{l}\text { MEAN } \\
\text { MINIMUM } \\
\text { MAXIMUM } \\
\text { NUMBER }\end{array}$ & $\begin{array}{r}12 \\
9 \\
15 \\
9\end{array}$ & $\begin{array}{l}5.6 \\
5.2 \\
5.2 \\
9\end{array}$ & $\begin{array}{l}2.0 \\
1.5 \\
2.4 \\
9\end{array}$ & $\begin{array}{c}17.5 \\
14.4 \\
24.2 \\
9\end{array}$ & $\begin{array}{l}0.07 \\
0.03 \\
0.24 \\
7\end{array}$ & $\begin{array}{l}0.05 \\
0.01 \\
0.12 \\
9\end{array}$ & $\begin{array}{r}109 \\
84 \\
161 \\
9\end{array}$ & $\begin{array}{r}13.8 \\
111 \\
159 \\
9\end{array}$ & $\begin{array}{l}0 \\
0 \\
0 \\
8\end{array}$ & $\begin{array}{l}0 \\
0 \\
0 \\
8\end{array}$ \\
\hline$X \mathbf{L}$ & $\begin{array}{l}\text { MEAN } \\
\text { MINIMUM } \\
\text { MAXIMUM } \\
\text { NUMUEH }\end{array}$ & $\begin{array}{r}13 \\
4 \\
16 \\
7\end{array}$ & $\begin{array}{l}5.6 \\
5.0 \\
6.3 \\
7\end{array}$ & $\begin{array}{l}3.0 \\
2.0 \\
4.4 \\
7\end{array}$ & $\begin{array}{c}23.0 \\
17.3 \\
41.9 \\
7\end{array}$ & $\begin{array}{l}0.16 \\
0.01 \\
0.65 \\
7\end{array}$ & $\begin{array}{l}0.03 \\
0.01 \\
0.07 \\
7\end{array}$ & $\begin{array}{r}120 \\
86 \\
190 \\
.7\end{array}$ & $\begin{array}{r}142 \\
115 \\
210 \\
7\end{array}$ & $\begin{array}{l}0 \\
0 \\
0 \\
\mathbf{6}\end{array}$ & $\begin{array}{l}0 \\
0 \\
3 \\
7\end{array}$ \\
\hline XM & $\begin{array}{l}\text { MEAN } \\
\text { MINIMUM } \\
\text { MAXIMUM } \\
\text { NUMBER }\end{array}$ & $\begin{array}{r}18 \\
16 \\
19 \\
8\end{array}$ & $\begin{array}{l}5.5 \\
5.0 \\
6.2 \\
8\end{array}$ & $\begin{array}{l}6.6 \\
1.2 \\
8.0 \\
8\end{array}$ & $\begin{array}{c}18.4 \\
10.1 \\
26.0 \\
8\end{array}$ & $\begin{array}{l}0.07 \\
0.02 \\
0.13 \\
8\end{array}$ & $\begin{array}{l}0.10 \\
0.01 \\
0.38 \\
8\end{array}$ & $\begin{array}{r}93 \\
71 \\
130 \\
8\end{array}$ & $\begin{array}{r}108 \\
86 \\
132 \\
8\end{array}$ & $\begin{array}{l}0 \\
0 \\
0 \\
7\end{array}$ & $\begin{array}{l}0 \\
0 \\
3 \\
7\end{array}$ \\
\hline XN & $\begin{array}{l}\text { MEAN } \\
\text { MINIMUM } \\
\text { MAXIMUM } \\
\text { NUMBER }\end{array}$ & $\begin{array}{r}13 \\
13 \\
13 \\
1\end{array}$ & $\begin{array}{l}5.0 \\
5.0 \\
5.0 \\
1\end{array}$ & $\begin{array}{l}1.6 \\
1.6 \\
1.6\end{array}$ & $\begin{array}{l}20.9 \\
20.9 \\
20.9 \\
1\end{array}$ & $\begin{array}{l}0.02 \\
0.02 \\
0.02 \\
1\end{array}$ & $\begin{array}{l}0.03 \\
0.03 \\
0.03 \\
1\end{array}$ & $\begin{array}{r}123 \\
123 \\
123 \\
1\end{array}$ & $\begin{array}{c}96 \\
96 \\
96 \\
1\end{array}$ & $\begin{array}{l}0 \\
0 \\
0 \\
1\end{array}$ & $\begin{array}{l}0 \\
0 \\
0 \\
1\end{array}$ \\
\hline$x a$ & $\begin{array}{l}\text { MEAN } \\
\text { MINIMUM } \\
\text { MAXIIMUM } \\
\text { NUMBER }\end{array}$ & $\begin{array}{r}13 \\
12 \\
15 \\
5\end{array}$ & $\begin{array}{l}5.9 \\
5.7 \\
5.0 \\
5\end{array}$ & $\begin{array}{l}2.7 \\
2.0 \\
5.0 \\
5\end{array}$ & $\begin{array}{l}25.4 \\
21.4 \\
32.7 \\
5\end{array}$ & $\begin{array}{l}0.08 \\
0.01 \\
0.29 \\
5\end{array}$ & $\begin{array}{l}0.02 \\
0.01 \\
0.04 \\
5\end{array}$ & $\begin{array}{r}103 \\
75 \\
140 \\
5\end{array}$ & $\begin{array}{r}136 \\
116 \\
147 \\
5\end{array}$ & $\begin{array}{l}0 \\
0 \\
0 \\
5\end{array}$ & $\begin{array}{l}0 \\
0 \\
0 \\
4\end{array}$ \\
\hline $\mathrm{XR}$ & $\begin{array}{l}\text { MEAN } \\
\text { MINIMUM } \\
\text { MAXIMUM } \\
\text { NUMBER }\end{array}$ & $\begin{array}{c}10 \\
10 \\
10 \\
1\end{array}$ & $\begin{array}{l}5 \cdot 3 \\
5 \cdot 3 \\
5 \cdot 3 \\
1\end{array}$ & $\begin{array}{l}1 \\
7.5 \\
7.5 \\
7.5\end{array}$ & $\begin{array}{l}7.4 \\
7.4 \\
7.4 \\
1\end{array}$ & $\begin{array}{l}0.03 \\
0.03 \\
0.03 \\
1\end{array}$ & $\begin{array}{l}0.06 \\
0.06 \\
0.06 \\
1\end{array}$ & $\begin{array}{r}26 \\
25 \\
26 \\
1\end{array}$ & $\begin{array}{r}45 \\
45 \\
45 \\
1\end{array}$ & $\begin{array}{l}0 \\
0 \\
0 \\
1\end{array}$ & $\begin{array}{l}0 \\
0 \\
0 \\
1\end{array}$ \\
\hline xs & $\begin{array}{l}\text { MEAN } \\
\text { MINIMUM } \\
\text { MAXIMUM } \\
\text { NUMBER }\end{array}$ & $\begin{array}{l}12 \\
11 \\
13 \\
10\end{array}$ & $\begin{array}{r}5.4 \\
4.8 \\
5.8 \\
10\end{array}$ & $\begin{array}{r}8.6 \\
7.8 \\
9.4 \\
10\end{array}$ & $\begin{array}{r}11.4 \\
0.0 \\
25.0 \\
10\end{array}$ & $\begin{array}{l}0.92 \\
0.13 \\
1.47 \\
10\end{array}$ & $\begin{array}{l}0.08 \\
0.01 \\
0.46 \\
7\end{array}$ & $\begin{array}{r}10 ! \\
71 \\
155 \\
10\end{array}$ & $\begin{array}{l}126 \\
106 \\
151 \\
10\end{array}$ & $\begin{array}{l}0 \\
0 \\
0 \\
8\end{array}$ & $\begin{array}{l}0 \\
0 \\
0 \\
9\end{array}$ \\
\hline
\end{tabular}


TABLE 22 (Continued)

1979 BNL ENVIRONMENTAL MONITORING MISCELLANEOUS ON SITE WELLS WA. IER QUALITY AND PURITY

\begin{tabular}{|c|c|c|c|c|c|c|c|c|c|c|c|}
\hline LOCATION & N SAMPLE & TEMPERATURE & $\mathrm{PH}$ & $\begin{array}{l}\text { OISSOLVEO } \\
\text { OXYGEN } \\
\text { (PPM) }\end{array}$ & $\begin{array}{l}\text { CHLORIDES } \\
\text { (PPM) }\end{array}$ & $\begin{array}{l}\text { NITRATE } \\
\text { NITROGEN } \\
\text { (PPM) }\end{array}$ & $\begin{array}{l}\text { TOTAL } \\
\text { PHOSPHOROUS } \\
\text { (PPM) }\end{array}$ & $\begin{array}{l}\text { DISSOL VED } \\
\text { SOL IOS } \\
\text { (PPM) }\end{array}$ & $\begin{array}{l}\text { CONOUC- } \\
\text { TIVITY } \\
\text { MICROMHO/CM }\end{array}$ & $\begin{array}{l}\text { COLIFORM } \\
\text { FECAL } \\
(/ 100 M L)\end{array}$ & $\begin{array}{c}\text { COLIFORM } \\
\text { TOTAL } \\
(100 \mathrm{ML})\end{array}$ \\
\hline$X W$ & $\begin{array}{l}\text { MEAN } \\
\text { MINIMUM } \\
\text { MAXIMUM } \\
\text { NUMBER }\end{array}$ & $\begin{array}{l}11 \\
11 \\
11 \\
1\end{array}$ & $\begin{array}{l}5.0 \\
5.0 \\
5.0 \\
1\end{array}$ & $\begin{array}{l}5.0 \\
5.0 \\
5.0 \\
1\end{array}$ & $\begin{array}{c}33.7 \\
33.7 \\
33.7 \\
1\end{array}$ & $\begin{array}{l}0.03 \\
0.03 \\
0.03 \\
1\end{array}$ & $\begin{array}{l}0.01 \\
0.01 \\
0.01 \\
1\end{array}$ & $\begin{array}{r}90 \\
90 \\
90 \\
1\end{array}$ & $\begin{array}{r}144 \\
144 \\
144 \\
1\end{array}$ & $\begin{array}{l}0 \\
0 \\
0 \\
1\end{array}$ & $\begin{array}{l}0 \\
0 \\
0 \\
1\end{array}$ \\
\hline$x x$ & $\begin{array}{l}\text { MEAN } \\
\text { MINIMUM } \\
\text { MAXIMUM } \\
\text { NUMBER }\end{array}$ & $\begin{array}{l}12 \\
11 \\
14 \\
11\end{array}$ & $\begin{array}{r}5.4 \\
5.0 \\
6.2 \\
11\end{array}$ & $\begin{array}{l}2.5 \\
1.6 \\
4.6 \\
10\end{array}$ & $\begin{array}{l}17.6 \\
13.1 \\
22.4 \\
10\end{array}$ & $\begin{array}{l}0.08 \\
0.00 \\
0.43 \\
10\end{array}$ & $\begin{array}{l}0.56 \\
0.01 \\
6.01 \\
11\end{array}$ & $\begin{array}{r}84 \\
53 \\
186 \\
10\end{array}$ & $\begin{array}{r}98 \\
86 \\
114 \\
10\end{array}$ & $\begin{array}{l}0 \\
0 \\
0 \\
9\end{array}$. & $\begin{array}{l}0 \\
0 \\
0 \\
9\end{array}$ \\
\hline$X Y$ & $\begin{array}{l}\text { MEAN } \\
\text { MINIMUM } \\
\text { MAXIMUM } \\
\text { NUMEER }\end{array}$ & $\begin{array}{c}14 \\
14 \\
14 \\
1\end{array}$ & $\begin{array}{l}5.3 \\
5.3 \\
5.3 \\
1\end{array}$ & $\begin{array}{l}.8 \\
1.8 \\
1.8\end{array}$ & $\begin{array}{l}20.9 \\
20.9 \\
20.9 \\
1\end{array}$ & $\begin{array}{l}0.02 \\
0.02 \\
0.02 \\
1\end{array}$ & $\begin{array}{l}0.01 \\
0.01 \\
0.01 \\
1\end{array}$ & $\begin{array}{r}84 \\
84 \\
84 \\
1\end{array}$ & $\begin{array}{r}102 \\
102 \\
102 \\
1\end{array}$ & $\begin{array}{l}0 \\
0 \\
0 \\
1\end{array}$ & $\begin{array}{l}0 \\
0 \\
0 \\
1\end{array}$ \\
\hline$x z$ & $\begin{array}{l}\text { MEAN } \\
\text { MINIMUM } \\
\text { MAXIMUM } \\
\text { INUMEER }\end{array}$ & $\begin{array}{l}11 \\
11 \\
11 \\
1\end{array}$ & $\begin{array}{l}5.8 \\
5.8 \\
5.8 \\
1\end{array}$ & $\begin{array}{l}4.6 \\
4.6 \\
4.6 \\
1\end{array}$ & $\begin{array}{l}27.5 \\
27.5 \\
27.5 \\
1\end{array}$ & $\begin{array}{l}0.26 \\
0.26 \\
0.26 \\
1\end{array}$ & $\begin{array}{l}0.02 \\
0.02 \\
0.02 \\
1\end{array}$ & $\begin{array}{r}146 \\
146 \\
146 \\
1\end{array}$ & $\begin{array}{r}149 \\
149 \\
149 \\
1\end{array}$ & $\begin{array}{l}0 \\
0 \\
0 \\
1\end{array}$ & $\begin{array}{l}0 \\
0 \\
0 \\
1\end{array}$ \\
\hline$x_{1}$ & $\begin{array}{l}\text { MEAN } \\
\text { MINIMUM } \\
\text { MAXIMUM } \\
\text { NUMBER }\end{array}$ & $\begin{array}{l}8 \\
8 \\
8 \\
1\end{array}$ & $\begin{array}{l}5.0 \\
5.0 \\
5.0 \\
1\end{array}$ & $\begin{array}{l}6.4 \\
6.4 \\
6.4 \\
1\end{array}$ & $\begin{array}{l}6.9 \\
6.9 \\
6.9 \\
1\end{array}$ & $\begin{array}{l}0.03 \\
0.03 \\
0.03 \\
1\end{array}$ & $\begin{array}{l}0.03 \\
0.03 \\
0.03 \\
1\end{array}$ & $\begin{array}{r}97 \\
97 \\
97 \\
1\end{array}$ & $\begin{array}{r}45 \\
45 \\
45 \\
1\end{array}$ & $\begin{array}{l}0 \\
0 \\
0 \\
1\end{array}$ & $\begin{array}{l}0 \\
0 \\
0 \\
1\end{array}$ \\
\hline$x$ & $\begin{array}{l}\text { MEAPI } \\
\text { IIIIIINII } \\
\text { MAXIMUM } \\
\text { NUMBER }\end{array}$ & $\begin{array}{l}11 \\
11 \\
11 \\
1\end{array}$ & $\begin{array}{l}5.2 \\
5.2 \\
5.2 \\
1\end{array}$ & $\begin{array}{l}2.0 \\
\text { E. } \\
2.0 \\
1\end{array}$ & $\begin{array}{l}0.1 \\
9.1 \\
9.1 \\
1\end{array}$ & $\begin{array}{l}0.02 \\
\text { u.je } \\
0.02 \\
1\end{array}$ & $\begin{array}{l}0.02 \\
0.02 \\
0.02 \\
1\end{array}$ & $\begin{array}{r}145 \\
145 \\
145 \\
1\end{array}$ & $\begin{array}{r}14 ! \\
14 ! \\
14 ! \\
1\end{array}$ & $\begin{array}{l}0 \\
U \\
0 \\
1\end{array}$ & $\begin{array}{l}0 \\
0 \\
0 \\
1\end{array}$ \\
\hline$\times 5$ & $\begin{array}{l}\text { MEAN } \\
\text { MINIMUM } \\
\text { MAXIMIJM } \\
\text { MUMBER }\end{array}$ & $\begin{array}{c}10 \\
10 \\
10 \\
1\end{array}$ & $\begin{array}{l}5.6 \\
5.6 \\
5.6 \\
1\end{array}$ & $\begin{array}{l}8.0 \\
8.0 \\
8.0 \\
1\end{array}$ & $\begin{array}{l}9.3 \\
9.3 \\
9.3 \\
1\end{array}$ & $\begin{array}{l}0.47 \\
0.47 \\
0.47 \\
1\end{array}$ & $\begin{array}{l}0.02 \\
0.02 \\
0.02 \\
1\end{array}$ & $\begin{array}{r}54 \\
54 \\
54 \\
1\end{array}$ & $\begin{array}{c}79 \\
79 \\
79 \\
1\end{array}$ & $\begin{array}{l}0 \\
0 \\
0 \\
1\end{array}$ & $\begin{array}{l}0 \\
0 \\
0 \\
1\end{array}$ \\
\hline SA & $\begin{array}{l}\text { MEAN } \\
\text { MINIMUM } \\
\text { MAXIMUM } \\
\text { NUMBER }\end{array}$ & $\begin{array}{l}14 \\
14 \\
14 \\
1\end{array}$ & $\begin{array}{l}5.5 \\
5.5 \\
5.5 \\
1\end{array}$ & $\begin{array}{l}6.4 \\
6.4 \\
6.4 \\
1\end{array}$ & $\begin{array}{c}45.0 \\
46.0 \\
46.0 \\
1\end{array}$ & $\begin{array}{l}2.51 \\
2.51 \\
2.51 \\
1\end{array}$ & $\begin{array}{l}0.0 ! \\
0.0 ! \\
0.01 \\
1\end{array}$ & $\begin{array}{r}139 \\
139 \\
139 \\
1\end{array}$ & $\begin{aligned} 212 \\
212 \\
215 \\
1\end{aligned}$ & $\begin{array}{l}0 \\
0 \\
0 \\
1\end{array}$ & $\begin{array}{l}0 \\
0 \\
0 \\
1\end{array}$ \\
\hline SC & $\begin{array}{l}\text { MEAN } \\
\text { MINIMUM } \\
\text { MAXIMUM } \\
\text { NUMBER }\end{array}$ & $\begin{array}{r}12 \\
12 \\
12 \\
1\end{array}$ & $\begin{array}{l}6.1 \\
6.1 \\
5.1 \\
1\end{array}$ & $\begin{array}{l}7.2 \\
7.2 \\
7.2 \\
1\end{array}$ & $\begin{array}{l}8.1 \\
8.1 \\
8.1 \\
1\end{array}$ & $\begin{array}{l}0.01 \\
0.01 \\
0.01 \\
1\end{array}$ & $\begin{array}{l}0.02 \\
0.02 \\
0.02 \\
1\end{array}$ & $\begin{array}{r}44 \\
44 \\
44 \\
1\end{array}$ & $\begin{array}{c}55 \\
56 \\
56 \\
1\end{array}$ & 0 & 0 \\
\hline SD & $\begin{array}{l}\text { MEAN } \\
\text { MIINII:UI1 } \\
\text { MAXIMUM } \\
\text { NUMBER }\end{array}$ & $\begin{array}{r}12 \\
12 \\
12 \\
1 \\
1\end{array}$ & $\begin{array}{l}5.2 \\
5: 2 \\
5.2 \\
1\end{array}$ & $\begin{array}{l}9.2 \\
9.2 \\
9.2 \\
1\end{array}$ & $\begin{array}{l}8.6 \\
0.0 \\
8.5 \\
1\end{array}$ & $\begin{array}{l}0.01 \\
0.01 \\
0.01 \\
1\end{array}$ & $\begin{array}{l}0.01 \\
0.01 \\
0.01 \\
1\end{array}$ & $\begin{array}{r}41 \\
41 \\
41 \\
1\end{array}$ & $\begin{array}{c}67 \\
67 \\
67 \\
1\end{array}$ & $\begin{array}{l}0 \\
\dot{u} \\
0 \\
1\end{array}$ & $\begin{array}{l}0 \\
U \\
0 \\
1\end{array}$ \\
\hline SE & $\begin{array}{l}\text { MEAN } \\
\text { MINIMUM } \\
\text { MAXIMUM } \\
\text { NUMBER }\end{array}$ & $\begin{array}{r}14 \\
13 \\
14 \\
3\end{array}$ & $\begin{array}{l}6.0 \\
5.6 \\
6.3 \\
3\end{array}$ & $\begin{array}{r}9.1 \\
8.5 \\
10.0 \\
3\end{array}$ & $\begin{array}{c}35.7 \\
28.7 \\
44.9\end{array}$ & $\begin{array}{l}1.39 \\
1.31 \\
1.46 \\
3\end{array}$ & $\begin{array}{l}0.03 \\
0.01 \\
0.07 \\
3\end{array}$ & $\begin{array}{r}152 \\
119 \\
169 \\
3\end{array}$ & $\begin{array}{r}197 \\
178 \\
205 \\
3\end{array}$ & $\begin{array}{l}0 \\
0 \\
0 \\
2\end{array}$ & $\begin{array}{l}0 \\
0 \\
0 \\
己\end{array}$ \\
\hline SG & $\begin{array}{l}\text { MEAN } \\
\text { MINIMUM } \\
\text { MAXIMUM } \\
\text { NUMBER }\end{array}$ & $\begin{array}{c}12 \\
12 \\
12 \\
1\end{array}$ & $\begin{array}{l}5.4 \\
5.4 \\
5.4 \\
1\end{array}$ & $\begin{array}{l}10.0 \\
10.0 \\
10.0 \\
1\end{array}$ & $\begin{array}{c}63.4 \\
63.4 \\
63.4 \\
1\end{array}$ & $\begin{array}{l}0.45 \\
0.45 \\
0.45 \\
1\end{array}$ & $\begin{array}{l}0.01 \\
0.01 \\
0.01 \\
1\end{array}$ & $\begin{array}{r}82 \\
82 \\
82 \\
1\end{array}$ & $\begin{array}{r}236 \\
236 \\
236 \\
1\end{array}$ & $\begin{array}{l}0 \\
0 \\
0 \\
1\end{array}$ & $\begin{array}{l}0 \\
0 \\
0 \\
1\end{array}$ \\
\hline 51 & $\begin{array}{l}\text { MEAN } \\
\text { MINIMUM } \\
\text { MAXIMUM } \\
\text { NUMBER }\end{array}$ & $\begin{array}{r}13 \\
13 \\
13 \\
1\end{array}$ & $\begin{array}{l}5.7 \\
5.7 \\
5.7 \\
1\end{array}$ & $\begin{array}{r}10.0 \\
10.0 \\
10.0 \\
1\end{array}$ & $\begin{array}{l}22 \cdot 3 \\
22.3 \\
22.3 \\
1\end{array}$ & $\begin{array}{l}0.62 \\
0.62 \\
0.62 \\
1\end{array}$ & $\begin{array}{l}0.01 \\
0.01 \\
0.01 \\
1\end{array}$ & $\begin{array}{r}87 \\
87 \\
87 \\
1\end{array}$ & $\left\{\begin{array}{c}114 \\
114 \\
114 \\
1\end{array}\right.$ & $\begin{array}{l}0 \\
0 \\
0 \\
1\end{array}$ & $\begin{array}{l}0 \\
0 \\
0 \\
1\end{array}$ \\
\hline 18 & $\begin{array}{l}\text { MEAIN } \\
\text { MINIMUM } \\
\text { MAXIMUM } \\
\text { NUMBER }\end{array}$ & $\begin{array}{l}12 \\
12 \\
12 \\
1\end{array}$ & $\begin{array}{l}4.7 \\
4.7 \\
4.7 \\
1\end{array}$ & $\begin{array}{l}2.6 \\
2.6 \\
2.6 \\
1\end{array}$ & $\begin{array}{l}11.8 \\
11.5 \\
11.5\end{array}$ & $\begin{array}{l}1.29 \\
2.29 \\
2.29\end{array}$ & $\begin{array}{l}0.01 \\
0.01 \\
0.01 \\
1\end{array}$ & $\begin{array}{l}121 \\
121 \\
121\end{array}$ & $\begin{array}{l}112 \\
112 \\
112 \\
112 \\
1\end{array}$ & $\begin{array}{l}0 \\
0 \\
n \\
1\end{array}$ & $\begin{array}{l}0 \\
0 \\
n \\
1\end{array}$ \\
\hline 17 & $\begin{array}{l}\text { MEAN } \\
\text { MINIMUM } \\
\text { PAXIMIUPI } \\
\text { NUMBER }\end{array}$ & 11 & $\begin{array}{l}5.8 \\
5.8 \\
5.8 \\
1\end{array}$ & $\begin{array}{l}9.8 \\
9.8 \\
9.8 \\
1\end{array}$ & $\begin{array}{l}12.1 \\
12.1 \\
12.1 \\
1\end{array}$ & $\begin{array}{l}0.01 \\
0.01 \\
0.01 \\
1\end{array}$ & $\begin{array}{l}0.01 \\
0.01 \\
u .01 \\
1\end{array}$ & $\begin{array}{c}100 \\
100 \\
100 \\
1\end{array}$ & $\begin{array}{c}68 \\
68 \\
b 8 \\
1\end{array}$ & $\begin{array}{l}0 \\
0 \\
u \\
1\end{array}$ & $\begin{array}{l}0 \\
0 \\
0 \\
1\end{array}$ \\
\hline टE & $\begin{array}{l}\text { MEAN } \\
\text { MINIMUM } \\
\text { MAXIMUM } \\
\text { NUMBER }\end{array}$ & $\begin{array}{r}11 \\
8 \\
14 \\
2\end{array}$ & $\begin{array}{l}5.4 \\
4.8 \\
6.0 \\
2\end{array}$ & $\begin{array}{l}7.3 \\
5.5 \\
9.0 \\
2\end{array}$ & $\begin{array}{c}56.7 \\
22.2 \\
91.1 \\
2\end{array}$ & $\begin{array}{l}0.13 \\
0.01 \\
0.26 \\
2\end{array}$ & $\begin{array}{l}0.03 \\
0.02 \\
0.04 \\
2\end{array}$ & $\begin{array}{r}132 \\
92 \\
17 ! \\
2\end{array}$ & $\begin{array}{r}202 \\
123 \\
281 \\
2\end{array}$ & $\begin{array}{l}0 \\
0 \\
0 \\
2\end{array}$ & $\begin{array}{l}0 \\
0 \\
0 \\
2\end{array}$ \\
\hline $2 F$ & $\begin{array}{l}\text { MEAN } \\
\text { MINIMUM } \\
\text { MAXIMUM } \\
\text { NUMBER }\end{array}$ & $\begin{array}{r}15 \\
15 \\
15 \\
2\end{array}$ & $\begin{array}{l}6.0 \\
6.0 \\
6.0 \\
2\end{array}$ & $\begin{array}{l}9.3 \\
9.2 \\
+9.4 \\
2 .\end{array}$ & $\begin{array}{c}45.2 \\
12.4 \\
78.0 \\
2\end{array}$ & $\begin{array}{l}0.23 \\
0.19 \\
0.27 \\
2\end{array}$ & $\begin{array}{l}0.01 \\
0.01 \\
0.01 \\
2\end{array}$ & $\begin{array}{r}142 \\
9 ! \\
192 \\
2\end{array}$ & $\begin{array}{r}193 \\
88 \\
298 \\
2\end{array}$ & $\begin{array}{l}0 \\
0 \\
0 \\
2\end{array}$ & $\begin{array}{l}0 \\
0 \\
0 \\
?\end{array}$ \\
\hline Cיב & $\begin{array}{l}\text { MEAN } \\
\text { MINIMUM } \\
\text { MAXIMUM } \\
\text { INMMUI }\end{array}$ & $\begin{array}{l}21 \\
21 \\
21 \\
1\end{array}$ & $\begin{array}{l}5.7 \\
5.7 \\
5.7 \\
1\end{array}$ & $\begin{array}{l}2.5 \\
2.6 \\
2.5 \\
1\end{array}$ & $\begin{array}{c}26.2 \\
26.2 \\
26.2 \\
1\end{array}$ & $\begin{array}{l}0.09 \\
0.09 \\
0.09 \\
1\end{array}$ & $\begin{array}{l}0.26 \\
0.26 \\
0.26 \\
1\end{array}$ & $\begin{array}{r}10 ! \\
101 \\
101 \\
1\end{array}$ & $\begin{array}{r}143 \\
143 \\
143 \\
1\end{array}$ & $\begin{array}{l}0 \\
0 \\
0 \\
1\end{array}$ & $\begin{array}{l}0 \\
0 \\
0 \\
1\end{array}$ \\
\hline
\end{tabular}

Reference Standards - Table 29

Number: samples analyzed per year 
TABLE 23

1979 BNL ENVIRONMENTAL MONITORING WASTE MANAGEMENT AREA WELLS WATER OUAL, ITY AND PURITY

\begin{tabular}{|c|c|c|c|c|c|c|c|c|c|c|c|}
\hline LOCATION & V SAMPLE & TEMPERATURE & $\mathrm{PH}$ & $\begin{array}{l}\text { DISSOL VED } \\
\text { OXYGEN } \\
\text { (PPM) }\end{array}$ & $\begin{array}{l}\text { CHLORIDES } \\
\text { (PPM) }\end{array}$ & $\begin{array}{l}\text { Ni TRATE } \\
\text { Ni TROGEN } \\
\text { (PPM) }\end{array}$ & $\begin{array}{l}\text { TOTAL } \\
\text { PHOSPHOROUS } \\
\text { (PPM) }\end{array}$ & $\begin{array}{l}\text { DISSOLVED } \\
\text { SOL IDS } \\
\text { (PPM) }\end{array}$ & $\begin{array}{l}\text { CONDUC- } \\
\text { TIVITY } \\
\text { MICROMHO/CM }\end{array}$ & $\begin{array}{l}\text { COL IFORM } \\
\text { FECAL } \\
(/ 100 \mathrm{ML})\end{array}$ & $\begin{array}{l}\text { COL IFORM } \\
\text { TOTAL } \\
(1 / 00 \mathrm{ML})\end{array}$ \\
\hline WB & $\begin{array}{l}\text { MEAN } \\
\text { M!NIMUM } \\
\text { MAXIMUM } \\
\text { NUMBER }\end{array}$ & $\begin{array}{r}14 \\
13 \\
15 \\
3\end{array}$ & $\begin{array}{l}5.4 \\
5.2 \\
5.5 \\
2\end{array}$ & $\begin{array}{l}6.0 \\
4.8 \\
7.0 \\
3\end{array}$ & $\begin{array}{l}8.2 \\
7.6 \\
8.6 \\
3\end{array}$ & $\begin{array}{l}2.51 \\
0.51 \\
5.25 \\
3\end{array}$ & $\begin{array}{l}2.01 \\
0.01 \\
6.01 \\
3\end{array}$ & $\begin{array}{r}50 \\
1 \\
94 \\
3\end{array}$ & $\begin{array}{r}105 \\
74 \\
124 \\
3\end{array}$ & $\begin{array}{l}0 \\
0 \\
0 \\
2\end{array}$ & $\begin{array}{l}0 \\
0 \\
0 \\
2\end{array}$ \\
\hline WC & $\begin{array}{l}\text { MEAN } \\
\text { MINIMUM } \\
\text { MAXIMUM } \\
\text { NIUMBER }\end{array}$ & $\begin{array}{r}15 \\
15 \\
15 \\
1\end{array}$ & $\begin{array}{l}5.2 \\
5.2 \\
5.2 \\
1\end{array}$ & $\begin{array}{l}7.4 \\
7.4 \\
7.4 \\
1\end{array}$ & $\begin{array}{l}6.6 \\
6.6 \\
6.6 \\
1\end{array}$ & $\begin{array}{l}4.85 \\
4.85 \\
4.85 \\
1\end{array}$ & $\begin{array}{l}0.02 \\
0.02 \\
0.02 \\
1\end{array}$ & $\begin{array}{l}75 \\
75 \\
75 \\
1\end{array}$ & $\begin{array}{c}104 \\
104 \\
104 \\
1\end{array}$ & $\begin{array}{l}0 \\
0 \\
0 \\
1\end{array}$ & $\begin{array}{l}0 \\
0 \\
0 \\
1\end{array}$ \\
\hline WD & $\begin{array}{l}\text { MEAN } \\
\text { MINIMUM } \\
\text { MAXIMUM } \\
\text { NUMBER }\end{array}$ & $\begin{array}{l}12 \\
10 \\
14 \\
5\end{array}$ & $\begin{array}{l}5.2 \\
4.8 \\
5.6 \\
5\end{array}$ & $\begin{array}{l}8.8 \\
8.4 \\
9.4 \\
5\end{array}$ & $\begin{array}{l}5.7 \\
4.0 \\
7.1 \\
5\end{array}$ & $\begin{array}{l}1.03 \\
0.66 \\
1.70 \\
5\end{array}$ & $\begin{array}{l}0.07 \\
0.02 \\
0.20 \\
5\end{array}$ & $\begin{array}{r}78 \\
70 \\
98 \\
5\end{array}$ & $\begin{array}{r}111 \\
100 \\
120 \\
5\end{array}$ & $\begin{array}{l}0 \\
0 \\
0 \\
1\end{array}$ & $\begin{array}{l}0 \\
0 \\
0 \\
1\end{array}$ \\
\hline WE & $\begin{array}{l}\text { MEAN } \\
\text { MINIMUM } \\
\text { MAXIMUM } \\
\text { NUMBER }\end{array}$ & $\begin{array}{l}13 \\
13 \\
13 \\
1\end{array}$ & $\begin{array}{l}5.7 \\
5.7 \\
5.7 \\
i\end{array}$ & $\begin{array}{l}8.4 \\
8.4 \\
8.4 \\
1\end{array}$ & $\begin{array}{l}2.5 \\
2.5 \\
2.5 \\
1\end{array}$ & $\begin{array}{l}0.01 \\
0.01 \\
0.01 \\
1\end{array}$ & $\begin{array}{l}0.02 \\
0.02 \\
0.02 \\
1\end{array}$ & $\begin{array}{r}39 \\
39 \\
39 \\
1\end{array}$ & $\begin{array}{r}44 \\
44 \\
44 \\
1\end{array}$ & 0 & 0 \\
\hline WJ & $\begin{array}{l}\text { MEAN } \\
\text { MINIMUM } \\
\text { MAXIMUM } \\
\text { NUMBER }\end{array}$ & $\begin{array}{r}13 \\
13 \\
13 \\
1\end{array}$ & $\begin{array}{l}5.2 \\
5.2 \\
5.2 \\
1\end{array}$ & $\begin{array}{l}7.0 \\
7.0 \\
7.0 \\
1\end{array}$ & $\begin{array}{l}9.1 \\
9.1 \\
9.1 \\
1\end{array}$ & $\begin{array}{l}0.60 \\
0.60 \\
0.60 \\
1\end{array}$ & $\begin{array}{l}0.02 \\
0.02 \\
0.02 \\
1\end{array}$ & $\begin{array}{c}70 \\
70 \\
70 \\
1\end{array}$ & $\begin{array}{l}95 \\
95 \\
95 \\
1\end{array}$ & 0 & 0 \\
\hline WK & $\begin{array}{l}\text { MEAN } \\
\text { MINIMUM } \\
\text { MAXIMUM } \\
\text { NUMBER }\end{array}$ & $\begin{array}{r}11 \\
10 \\
12 \\
5\end{array}$ & $\begin{array}{l}5.4 \\
5.2 \\
5.7 \\
5\end{array}$ & $\begin{array}{l}8.5 \\
8.0 \\
9.4 \\
5\end{array}$ & $\begin{array}{l}7.9 \\
5.6 \\
9.2 \\
4\end{array}$ & $\begin{array}{l}1.04 \\
0.62 \\
1.54 \\
5\end{array}$ & $\begin{array}{l}0.03 \\
0.01 \\
0.06 \\
5\end{array}$ & $\begin{array}{r}66 \\
58 \\
90 \\
5\end{array}$ & $\begin{array}{r}94 \\
86 \\
109 \\
5\end{array}$ & $\begin{array}{l}0 \\
0 \\
0 \\
4\end{array}$ & $\begin{array}{l}0 \\
0 \\
0 \\
4\end{array}$ \\
\hline WL & $\begin{array}{l}\text { MEAN } \\
\text { MINIMUM } \\
\text { MAXIMUM } \\
\text { NUMBER }\end{array}$ & $\begin{array}{r}11 \\
10 \\
13 \\
5\end{array}$ & $\begin{array}{l}5.3 \\
4.8 \\
5.8 \\
5\end{array}$ & $\begin{array}{l}9.5 \\
9.0 \\
9.8 \\
5\end{array}$ & $\begin{array}{l}3.5 \\
1.5 \\
4.5 \\
5\end{array}$ & $\begin{array}{l}1.66 \\
0.25 \\
2.42 \\
5\end{array}$ & $\begin{array}{l}0.03 \\
0.01 \\
0.05 \\
5\end{array}$ & $\begin{array}{r}75 \\
63 \\
83 \\
4\end{array}$ & $\begin{array}{r}94 \\
80 \\
104 \\
5\end{array}$ & $\begin{array}{l}0 \\
0 \\
0 \\
1\end{array}$ & $\begin{array}{l}0 \\
0 \\
0 \\
1\end{array}$ \\
\hline WM & $\begin{array}{l}\text { MEAN } \\
\text { MINIMUM } \\
\text { MAXIMUM } \\
\text { NUMBER }\end{array}$ & $\begin{array}{c}14 \\
14 \\
14 \\
1\end{array}$ & $\begin{array}{l}6.1 \\
5.1 \\
6.1 \\
1\end{array}$ & $\begin{array}{l}6.4 \\
6.4 \\
6.4 \\
1\end{array}$ & $\begin{array}{l}8.1 \\
8.1 \\
8.1 \\
1\end{array}$ & $\begin{array}{l}0.44 \\
0.44 \\
0.44 \\
1\end{array}$ & $\begin{array}{l}0.02 \\
0.02 \\
0.02 \\
1\end{array}$ & $\begin{array}{l}101 \\
101 \\
101 \\
1\end{array}$ & $\begin{array}{c}153 \\
153 \\
153 \\
1\end{array}$ & 0 & 0 \\
\hline Wiv & $\begin{array}{l}\text { MEAN } \\
\text { MINIMUM } \\
\text { MAXIMUM } \\
\text { NUMBER }\end{array}$ & $\begin{array}{c}13 \\
13 \\
13 \\
1\end{array}$ & $\begin{array}{l}5.9 \\
5.9 \\
5.9 \\
1\end{array}$ & $\begin{array}{l}7.0 \\
7.0 \\
7.0 \\
1\end{array}$ & $\begin{array}{l}9.1 \\
9.1 \\
9.1 \\
1\end{array}$ & $\begin{array}{l}0.13 \\
0.13 \\
0.13 \\
1\end{array}$ & $\begin{array}{l}0.02 \\
0.02 \\
0.02 \\
1\end{array}$ & $\begin{array}{c}95 \\
95 \\
95 \\
1\end{array}$ & $\begin{array}{r}148 \\
148 \\
148 \\
1\end{array}$ & 0 & 0 \\
\hline WV & $\begin{array}{l}\text { MEAN } \\
\text { MINIMUM } \\
\text { MAXIMUM } \\
\text { NUMBER }\end{array}$ & $\begin{array}{l}11 \\
11 \\
11 \\
1\end{array}$ & $\begin{array}{l}6.0 \\
6.0 \\
6.0 \\
1\end{array}$ & $\begin{array}{l}7.6 \\
7.6 \\
7.6 \\
1\end{array}$ & $\begin{array}{l}2.0 \\
2.0 \\
2.0 \\
1\end{array}$ & $\begin{array}{l}0.01 \\
0.01 \\
0.01 \\
1\end{array}$ & $\begin{array}{l}0.02 \\
0.02 \\
0.02 \\
1\end{array}$ & $\begin{array}{r}28 \\
28 \\
28 \\
1\end{array}$ & $\begin{array}{c}39 \\
39 \\
39 \\
1\end{array}$ & $\begin{array}{l}0 \\
0 \\
0 \\
1\end{array}$ & $\begin{array}{l}0 \\
0 \\
0 \\
1\end{array}$ \\
\hline WW & $\begin{array}{l}\text { MEAN } \\
\text { MINIMUM } \\
\text { MAXIMUM } \\
\text { NUMBER }\end{array}$ & $\begin{array}{ll}1 & 1 \\
11 \\
11 \\
1\end{array}$ & $\begin{array}{l}5.6 \\
5.6 \\
5.6 \\
1\end{array}$ & $\begin{array}{l}8.8 \\
8.8 \\
8.8 \\
1\end{array}$ & $\begin{array}{c}14.6 \\
14.6 \\
14.6 \\
1\end{array}$ & $\begin{array}{l}0.01 \\
0.01 \\
0.01 \\
1\end{array}$ & $\begin{array}{l}0.02 \\
0.02 \\
0.02 \\
1\end{array}$ & $\begin{array}{l}70 \\
70 \\
70 \\
1\end{array}$ & $\begin{array}{r}95 \\
95 \\
95 \\
1\end{array}$ & $\begin{array}{l}0 \\
0 \\
0 \\
1\end{array}$ & $\begin{array}{l}0 \\
0 \\
0 \\
1\end{array}$ \\
\hline$W X$ & $\begin{array}{l}\text { MEAN } \\
\text { MINIMUM } \\
\text { MAXXIMUM } \\
\text { NUMBER }\end{array}$ & $\begin{array}{r}12 \\
15 \\
15 \\
15\end{array}$ & $\begin{array}{l}5.9 \\
5.9 \\
5.9 \\
1\end{array}$ & $\begin{array}{l}\text { B. } \\
\text { B. } \\
\text { U. U } \\
1\end{array}$ & $\begin{array}{l}9.6 \\
9.6 \\
9.6 \\
1\end{array}$ & $\begin{array}{l}0.05 \\
0.05 \\
\text { U. } \\
1\end{array}$ & $\begin{array}{l}0.02 \\
0.02 \\
U .02 \\
1\end{array}$ & $\begin{array}{c}54 \\
54 \\
54 \\
1\end{array}$ & $\begin{array}{l}69 \\
69 \\
89 \\
1\end{array}$ & $\begin{array}{l}0 \\
0 \\
0 \\
1\end{array}$ & $\begin{array}{l}0 \\
0 \\
0 \\
1\end{array}$ \\
\hline WZ & $\begin{array}{l}\text { MEAN } \\
\text { MINIMUM } \\
\text { MAXIMUM } \\
\text { NUMBER }\end{array}$ & $\begin{array}{l}11 \\
11 \\
1 i \\
1\end{array}$ & $\begin{array}{l}5.7 \\
5.7 \\
5.7 \\
1\end{array}$ & $\begin{array}{l}7.4 \\
7.4 \\
7.4 \\
1\end{array}$ & $\begin{array}{l}6.6 \\
6.6 \\
6.6 \\
1\end{array}$ & $\begin{array}{l}0.01 \\
0.01 \\
0.01 \\
1\end{array}$ & $\begin{array}{l}0.02 \\
0.02 \\
0.02 \\
1\end{array}$ & $\begin{array}{r}54 \\
54 \\
54 \\
1\end{array}$ & $\begin{array}{l}81 \\
81 \\
81 \\
1\end{array}$ & $\begin{array}{l}0 \\
0 \\
0 \\
1\end{array}$ & 0 \\
\hline WI & $\begin{array}{l}\text { MEAN } \\
\text { MINIMUM } \\
\text { MAXIMUM } \\
\text { NUMBER }\end{array}$ & $\begin{array}{r}11 \\
10 \\
13 \\
5\end{array}$ & $\begin{array}{l}5.4 \\
5.0 \\
5.8 \\
5\end{array}$ & $\begin{array}{l}9.3 \\
8.0 \\
9.8 \\
5\end{array}$ & $\begin{array}{l}3.9 \\
3.3 \\
4.5 \\
5\end{array}$ & $\begin{array}{l}0.83 \\
0.13 \\
1.49 \\
5\end{array}$ & $\begin{array}{l}0.02 \\
0.01 \\
0.04 \\
5\end{array}$ & $\begin{array}{r}59 \\
38 \\
92 \\
3\end{array}$ & $\begin{array}{r}55 \\
45 \\
62 \\
5\end{array}$ & $\begin{array}{l}0 \\
0 \\
0 \\
1\end{array}$ & $\begin{array}{l}0 \\
0 \\
0 \\
1\end{array}$ \\
\hline W3 & $\begin{array}{l}\text { PCANA } \\
\text { MINIMUM } \\
\text { MAXIMUM } \\
\text { NUMBER }\end{array}$ & $\begin{array}{r}11 \\
11 \\
11 \\
1\end{array}$ & $\begin{array}{l}5.1 \\
5.1 \\
5.1 \\
1\end{array}$ & $\begin{array}{l}7.0 \\
7.0 \\
7.0 \\
1\end{array}$ & $\begin{array}{l}7.9 \\
7.9 \\
7.9 \\
1\end{array}$ & $\begin{array}{l}3.82 \\
3.82 \\
3.82 \\
1\end{array}$ & $\begin{array}{l}0.01 \\
0.01 \\
0.01 \\
1\end{array}$ & $\begin{array}{c}83 \\
83 \\
93 \\
1\end{array}$ & $\begin{array}{l}101 \\
101 \\
101 \\
1\end{array}$ & $\begin{array}{l}n \\
0 \\
0 \\
1\end{array}$ & $\begin{array}{l}n \\
0 \\
0 \\
1\end{array}$ \\
\hline 14 & $\begin{array}{l}\text { MEAN } \\
\text { MINIMUM } \\
\text { MAXIMUM } \\
\text { NUMBER }\end{array}$ & $\begin{array}{l}11 \\
11 \\
11 \\
1\end{array}$ & $\begin{array}{l}5.3 \\
5.3 \\
5.3 \\
1\end{array}$ & $\begin{array}{l}10.0 \\
10.0 \\
10.0 \\
1\end{array}$ & $\begin{array}{l}4.0 \\
4.0 \\
4.0 \\
1\end{array}$ & $\begin{array}{l}0.05 \\
0.05 \\
0.05 \\
1\end{array}$ & $\begin{array}{l}0.01 \\
0.01 \\
0.01 \\
1\end{array}$ & $\begin{array}{r}49 \\
49 \\
49 \\
1\end{array}$ & $\begin{array}{r}39 \\
39 \\
39 \\
1\end{array}$ & $\begin{array}{l}0 \\
0 \\
0 \\
1\end{array}$ & $\begin{array}{l}0 \\
0 \\
0 \\
1\end{array}$ \\
\hline W5 & $\begin{array}{l}\text { MEAN } \\
\text { MINIMUM } \\
\text { MAXIMUM } \\
\text { NUMBER }\end{array}$ & $\begin{array}{l}11 \\
11 \\
11 \\
1\end{array}$ & $\begin{array}{l}5.0 \\
5.0 \\
5.0 \\
1\end{array}$ & $\begin{array}{l}10.0 \\
10.0 \\
10.0 \\
1\end{array}$ & $\begin{array}{l}5.4 \\
5.4 \\
5.4 \\
1\end{array}$ & $\begin{array}{l}0.05 \\
0.05 \\
0.05 \\
1\end{array}$ & $\begin{array}{l}0.01 \\
0.01 \\
0.01 \\
1\end{array}$ & $\begin{array}{r}40 \\
40 \\
40 \\
1\end{array}$ & $\begin{array}{r}46 \\
46 \\
46 \\
1\end{array}$ & 0 & 0 \\
\hline
\end{tabular}


TABLE 23 (Continued)

1979 BNL ENVIRONMENTAL MONITORING LANDFILL AREA WELLS WATER QUAL

\begin{tabular}{|c|c|c|c|c|c|c|c|c|c|c|c|}
\hline LOCATION & N SAMPLE & TEMFERATURE & $\mathrm{PH}$ & $\begin{array}{l}\text { DISSOLVED } \\
\text { OXYGEN } \\
\text { (PPM) }\end{array}$ & $\begin{array}{l}\text { CHLORIDES } \\
\text { (PPM) }\end{array}$ & $\begin{array}{l}\text { NI TRATE } \\
\text { NI TROGEN } \\
\text { (PPM) }\end{array}$ & $\begin{array}{l}\text { TOTAL } \\
\text { PHOSPHOROUS } \\
\text { (PPM) }\end{array}$ & $\begin{array}{l}\text { DISSOL VEO } \\
\text { SOL IDS } \\
\text { (PPM) }\end{array}$ & $\begin{array}{l}\text { CONDUC- } \\
\text { TIVITY } \\
\text { MICROMHO/CM }\end{array}$ & $\begin{array}{l}\text { COLIFORM } \\
\text { FECAL } \\
(1100 \mathrm{ML})\end{array}$ & $\begin{array}{l}\text { COL JFORM } \\
\text { TOTAL } \\
(1100 \mathrm{ML})\end{array}$ \\
\hline W7 & $\begin{array}{l}\text { MEAN } \\
\text { MINIMUM } \\
\text { MAXIMUM } \\
\text { NUMBER }\end{array}$ & $\begin{array}{l}11 \\
11 \\
11\end{array}$ & $\begin{array}{l}5.1 \\
5.1 \\
5.1 \\
1\end{array}$ & $\begin{array}{l}7.0 \\
7.0 \\
7.0 \\
1\end{array}$ & $\begin{array}{l}10.9 \\
10.9 \\
10.9 \\
1\end{array}$ & $\begin{array}{l}0.54 \\
0.54 \\
0.64 \\
1\end{array}$ & $\begin{array}{l}0.01 \\
0.01 \\
0.01 \\
1\end{array}$ & $\begin{array}{l}60 \\
60 \\
60 \\
1\end{array}$ & $\begin{array}{l}89 \\
89 \\
89 \\
1\end{array}$ & $\begin{array}{l}0 \\
0 \\
0 \\
1\end{array}$ & $\begin{array}{l}0 \\
0 \\
0 \\
1\end{array}$ \\
\hline พ8 & $\begin{array}{l}\text { MEAN } \\
\text { MINIMUM } \\
\text { MAXIMUM } \\
\text { NUMBER }\end{array}$ & $\begin{array}{l}1 ! \\
11 \\
1 !\end{array}$ & $\begin{array}{l}5.0 \\
5.0 \\
5.0 \\
1\end{array}$ & $\begin{array}{l}9.2 \\
9.2 \\
9.2 \\
1\end{array}$ & $\begin{array}{l}5.0 \\
5.0 \\
5.0 \\
1\end{array}$ & $\begin{array}{l}0.05 \\
0.05 \\
0.05 \\
1\end{array}$ & $\begin{array}{l}0.01 \\
0.01 \\
0.01 \\
1\end{array}$ & $\begin{array}{r}43 \\
43 \\
43 \\
1\end{array}$ & $\begin{array}{r}47 \\
47 \\
47 \\
1\end{array}$ & 0 & 0 \\
\hline 18 & $\begin{array}{l}\text { MEAN } \\
\text { M! M!| M! IM! } \\
\text { MAX!MUM } \\
\text { NUMBER }\end{array}$ & $\begin{array}{c}12 \\
10 \\
10 \\
1\end{array}$ & $\begin{array}{l}4.8 \\
1.9 \\
1.8 \\
1\end{array}$ & $\begin{array}{l}10.0 \\
10.0 \\
10.0 \\
1\end{array}$ & $\begin{array}{l}6.4 \\
6.11 \\
6.11 \\
1\end{array}$ & $\begin{array}{l}0.05 \\
0.05 \\
0.05 \\
1\end{array}$ & $\begin{array}{l}0.01 \\
0.01 \\
0.01 \\
1\end{array}$ & $\begin{array}{c}44 \\
111 \\
111 \\
1\end{array}$ & $\begin{array}{l}61 \\
\mathbf{C} 1 \\
61 \\
1\end{array}$ & $\begin{array}{l}0 \\
0 \\
0 \\
1\end{array}$ & $\begin{array}{l}0 \\
0 \\
0 \\
1\end{array}$ \\
\hline 19 & $\begin{array}{l}\text { MEAN } \\
\text { MINIMUM } \\
\text { MAXIMUM } \\
\text { NUMBER }\end{array}$ & $\begin{array}{l}12 \\
12 \\
12 \\
1\end{array}$ & $\begin{array}{l}5.0 \\
5.0 \\
5.0 \\
1\end{array}$ & $\begin{array}{l}10.0 \\
10.0 \\
10.0 \\
1\end{array}$ & $\begin{array}{l}8.4 \\
B .4 \\
8.4 \\
1\end{array}$ & $\begin{array}{l}0.05 \\
0.05 \\
0.05 \\
1\end{array}$ & $\begin{array}{l}0.01 \\
0.01 \\
0.01 \\
1\end{array}$ & $\begin{array}{r}46 \\
40 \\
46 \\
1\end{array}$ & $\begin{array}{c}68 \\
68 \\
68 \\
1\end{array}$ & $\begin{array}{l}0 \\
0 \\
0 \\
1\end{array}$ & $\begin{array}{l}0 \\
0 \\
0 \\
1\end{array}$ \\
\hline HF & $\begin{array}{l}\text { MEAN } \\
\text { MINIMUM } \\
\text { MAXIMUM } \\
\text { NUMBER }\end{array}$ & $\begin{array}{r}12 \\
12 \\
12 \\
12 \\
5\end{array}$ & $\begin{array}{l}5.3 \\
4.6 \\
5.7 \\
2\end{array}$ & $\begin{array}{l}8.2 \\
8.0 \\
9.4 \\
2\end{array}$ & $\begin{array}{r}13.1 \\
6.5 \\
17.6 \\
2\end{array}$ & $\begin{array}{l}0.34 \\
0.05 \\
0.63 \\
2\end{array}$ & $\begin{array}{l}0.02 \\
0.01 \\
0.02 \\
2\end{array}$ & $\begin{array}{r}60 \\
47 \\
73 \\
2\end{array}$ & $\begin{array}{r}83 \\
76 \\
90 \\
2\end{array}$ & $\begin{array}{l}0 \\
0 \\
0 \\
1\end{array}$ & $\begin{array}{l}0 \\
0 \\
0 \\
1\end{array}$ \\
\hline WG & $\begin{array}{l}\text { MIAN } \\
\text { MINIMUM } \\
\text { MAXIMUM } \\
\text { NUMBER }\end{array}$ & $\begin{array}{r}13 \\
13 \\
13 \\
2\end{array}$ & $\begin{array}{l}5.4 \\
5.4 \\
5.4 \\
2\end{array}$ & $\begin{array}{l}5.0 \\
3.5 \\
8.0 \\
2\end{array}$ & $\begin{array}{l}7.3 \\
6.0 \\
8.6 \\
2\end{array}$ & $\begin{array}{l}0.17 \\
0.03 \\
0.31 \\
2\end{array}$ & $\begin{array}{l}0.03 \\
0.01 \\
0.04 \\
2\end{array}$ & $\begin{array}{r}41 \\
38 \\
43 \\
2\end{array}$ & $\begin{array}{r}58 \\
54 \\
81 \\
2\end{array}$ & $\begin{array}{l}0 \\
0 \\
0 \\
2\end{array}$ & $\begin{array}{l}0 \\
0 \\
0 \\
a \\
\text { cे }\end{array}$ \\
\hline WR & $\begin{array}{l}\text { MEAN } \\
\text { MINIMUM } \\
\text { MAXIMUM } \\
\text { NUMBER }\end{array}$ & $\begin{array}{l}15 \\
15 \\
15 \\
2\end{array}$ & $\begin{array}{l}6.4 \\
6.2 \\
6.5 \\
2\end{array}$ & $\begin{array}{l}2.6 \\
2.2 \\
3.0 \\
2\end{array}$ & $\begin{array}{r}9.6 \\
1.5 \\
17.7 \\
2\end{array}$ & $\begin{array}{l}0.39 \\
0.26 \\
0.51 \\
2\end{array}$ & $\begin{array}{l}0.02 \\
0.02 \\
0.02 \\
2\end{array}$ & $\begin{array}{r}445 \\
354 \\
536 \\
2\end{array}$ & $\begin{array}{r}1108 \\
1085 \\
1130 \\
2\end{array}$ & $\begin{array}{l}0 \\
0 \\
0 \\
1\end{array}$ & $\begin{array}{l}0 \\
0 \\
0 \\
1\end{array}$ \\
\hline W5 & $\begin{array}{l}\text { MEAN } \\
\text { MINIMUM } \\
\text { MAXIMUM } \\
\text { PUUMER }\end{array}$ & $\begin{array}{r}12 \\
10 \\
14 \\
11\end{array}$ & $\begin{array}{l}5.1 \\
1.4 \\
6.7 \\
1 .\end{array}$ & $\begin{array}{l}1.8 \\
1.6 \\
2.0 \\
2\end{array}$ & $\begin{array}{r}27.7 \\
2.6 \\
46.9 \\
4\end{array}$ & $\begin{array}{l}0.23 \\
0.05 \\
0.55 \\
4\end{array}$ & $\begin{array}{l}0.02 \\
0.01 \\
0.02 \\
4\end{array}$ & $\begin{array}{r}370 \\
233 \\
449 \\
4\end{array}$ & $\begin{array}{r}738 \\
670 \\
839 \\
4\end{array}$ & $\begin{array}{l}0 \\
0 \\
0 \\
3\end{array}$ & $\begin{array}{l}0 \\
u \\
0 \\
2\end{array}$ \\
\hline WT & $\begin{array}{l}\text { MEAN } \\
\text { MINIMUM } \\
\text { MAXIMUM } \\
\text { IUIIBER }\end{array}$ & $\begin{array}{r}12 \\
11 \\
13 \\
0\end{array}$ & $\begin{array}{l}6.2 \\
6.0 \\
6.4 \\
E\end{array}$ & $\begin{array}{l}3.3 \\
2.2 \\
4.4 \\
2\end{array}$ & $\begin{array}{r}9.9 \\
9.9 \\
10.6 \\
\varepsilon .\end{array}$ & $\begin{array}{l}0.11 \\
0.01 \\
0.20 \\
3\end{array}$ & $\begin{array}{l}0.02 \\
0.01 \\
0.02 \\
\dot{C}\end{array}$ & $\begin{array}{l}54 \\
56 \\
7 ! \\
2\end{array}$ & $\begin{array}{r}87 \\
84 \\
90 \\
2\end{array}$ & $\begin{array}{l}0 \\
0 \\
0 \\
\ddot{2}\end{array}$ & $\begin{array}{l}0 \\
0 \\
0 \\
2\end{array}$ \\
\hline ผ9 & $\begin{array}{l}\text { HEAN } \\
\text { MINIMUM } \\
\text { MAXIMUM } \\
\text { NUMBER }\end{array}$ & $\begin{array}{r}12 \\
-\quad 11 \\
13 \\
4\end{array}$ & $\begin{array}{l}\text { B. } \\
5.6 \\
6.7 \\
4\end{array}$ & $\begin{array}{l}2.5 \\
1.5 \\
3.5 \\
4\end{array}$ & $\begin{array}{r}16.8 \\
3.6 \\
52.0 \\
4\end{array}$ & $\begin{array}{l}0.30 \\
0.12 \\
0.83 \\
4\end{array}$ & $\begin{array}{l}0.01 \\
0.01 \\
0.02 \\
4\end{array}$ & $\begin{array}{r}419 \\
333 \\
588 \\
4\end{array}$ & $\begin{array}{r}934 \\
815 \\
1005 \\
4\end{array}$ & $\begin{array}{l}0 \\
0 \\
0 \\
4\end{array}$ & $\begin{array}{l}0 \\
0 \\
u \\
4\end{array}$ \\
\hline IK. & $\begin{array}{l}\text { MEAN } \\
\text { MINIMUM } \\
\text { MAXIMUM } \\
\text { NUMBER }\end{array}$ & $\begin{array}{r}14 \\
13 \\
15 \\
3\end{array}$ & $\begin{array}{l}6.3 \\
6.2 \\
6.6 \\
3\end{array}$ & $\begin{array}{l}1.3 \\
1.0 \\
1.4 \\
3\end{array}$ & $\begin{array}{r}33.7 \\
2.0 \\
93.4 \\
3\end{array}$ & $\begin{array}{l}0.30 \\
0.25 \\
0.36 \\
3\end{array}$ & $\begin{array}{l}0.01 \\
0.01 \\
0.01 \\
3\end{array}$ & $\begin{array}{r}474 \\
454 \\
496 \\
3\end{array}$ & $\begin{array}{r}983 \\
905 \\
1049 \\
3\end{array}$ & $\begin{array}{l}0 \\
0 \\
0 \\
2\end{array}$ & $\begin{array}{l}0 \\
0 \\
0 \\
?\end{array}$ \\
\hline '2A & $\begin{array}{l}\text { MEAN } \\
\text { MINIMUM } \\
\text { MAX IIAUI'\| } \\
\text { NUMBER }\end{array}$ & $\begin{array}{l}11 \\
11 \\
11 \\
2\end{array}$ & $\begin{array}{l}5.6 \\
5.5 \\
.5 .7 \\
2\end{array}$ & $\begin{array}{r}9.9 \\
9.9 \\
10.0 \\
2\end{array}$ & $\begin{array}{l}6.9 \\
5.1 \\
8.6 \\
2\end{array}$ & $\begin{array}{l}0.02 \\
0.01 \\
0.03 \\
2\end{array}$ & $\begin{array}{l}0.01 \\
0.01 \\
0.01 \\
己\end{array}$ & $\begin{array}{r}40 \\
37 \\
43 \\
2\end{array}$ & $\begin{array}{r}55 \\
50 \\
59 \\
2\end{array}$ & $\begin{array}{l}0 \\
0 \\
0 \\
2\end{array}$ & $\begin{array}{l}1 \\
0 \\
1 \\
2\end{array}$ \\
\hline ट日 & $\begin{array}{l}\text { MEAN } \\
\text { MINIMUM } \\
\text { MAXIMUM } \\
\text { NUMBER }\end{array}$ & $\begin{array}{l}11 \\
10 \\
11 \\
2\end{array}$ & $\begin{array}{l}5.3 \\
5.0 \\
5.5 \\
2\end{array}$ & $\begin{array}{l}10.1 \\
10.0 \\
10.2 \\
2\end{array}$ & $\begin{array}{r}8.6 \\
5.6 \\
11.6 \\
2\end{array}$ & $\begin{array}{l}0.08 \\
0.02 \\
0.09 \\
2\end{array}$ & $\begin{array}{l}0.03 \\
0.02 \\
0.03 \\
\text {. }\end{array}$ & $\begin{array}{r}46 \\
44 \\
47 \\
2\end{array}$ & $\begin{array}{r}69 \\
62 \\
79 \\
2\end{array}$ & $\begin{array}{l}0 \\
0 \\
0 \\
1\end{array}$ & $\begin{array}{l}J \\
0 \\
0 \\
1\end{array}$ \\
\hline टC & $\begin{array}{l}\text { MEAN } \\
\text { MINIMUM } \\
\text { MAX I MUUM } \\
\text { NUMBER }\end{array}$ & $\begin{array}{r}13 \\
13 \\
13 \\
? \\
?\end{array}$ & $\begin{array}{l}6 \cdot 3 \\
6 \cdot 2 \\
5 \cdot 3 \\
2\end{array}$ & $\begin{array}{l}2.0 \\
1.8 \\
2.4 \\
2\end{array}$ & $\begin{array}{l}2.0 \\
1: 5 \\
2.5 \\
2\end{array}$ & $\begin{array}{l}0.34 \\
0.39 \\
0.38 \\
?\end{array}$ & $\begin{array}{l}0.05 \\
0.03 \\
0.07 \\
2\end{array}$ & $\begin{array}{r}639 \\
839 \\
639 \\
1\end{array}$ & $\begin{array}{r}1213 \\
1170 \\
1255 \\
2\end{array}$ & $\begin{array}{l}0 \\
n \\
0 \\
2\end{array}$ & $\begin{array}{l}0 \\
0 \\
0 \\
己\end{array}$ \\
\hline 20 & $\begin{array}{l}\text { MEAN } \\
\text { MINIMUIA } \\
\text { MAXIMUM } \\
\text { NUMBER }\end{array}$ & $\begin{array}{r}13 \\
13 \\
13 \\
2\end{array}$ & $\begin{array}{l}6.2 \\
6.0 \\
6.4 \\
2\end{array}$ & $\begin{array}{l}2.0 \\
2.0 \\
2.0 \\
2\end{array}$ & $\begin{array}{c}25.9 \\
23.7 \\
28.0 \\
2\end{array}$ & $\begin{array}{l}0.21 \\
0.19 \\
0.23 \\
2\end{array}$ & $\begin{array}{l}0.04 \\
0.01 \\
0.06 \\
2\end{array}$ & $\begin{array}{r}326 \\
326 \\
326 \\
1\end{array}$ & $\begin{array}{r}870 \\
800 \\
940 \\
2\end{array}$ & $\begin{array}{l}0 \\
0 \\
n \\
2\end{array}$ & $\begin{array}{l}0 \\
0 \\
0 \\
2\end{array}$ \\
\hline
\end{tabular}


TABLE 23 (Continued)

1979 BNL ENVIRONMENTAL MONITORING FORMER DUMP AREA WELLS WATER QUALITY AND PURI TY

\begin{tabular}{|c|c|c|c|c|c|c|c|c|c|c|c|}
\hline LOCATION & $\checkmark$ SAMPLE & TEMPERATURE & PH & $\begin{array}{l}\text { O1SSOL VED } \\
\text { OXYGEN } \\
\text { (PPM) }\end{array}$ & $\begin{array}{l}\text { CHLOR IDES } \\
\text { (PPM) }\end{array}$ & $\begin{array}{l}\text { NITRATE } \\
\text { NITROGEN } \\
\text { (PPM) }\end{array}$ & $\begin{array}{l}\text { TOTAL } \\
\text { PHOSPHOROUS } \\
\text { (PPM) }\end{array}$ & $\begin{array}{l}\text { DISSOLVEO } \\
\text { SOLIDS } \\
\text { (PPM) }\end{array}$ & $\begin{array}{l}\text { CONDUC- } \\
\text { IIVITY } \\
\text { MICROMHO/CM }\end{array}$ & $\begin{array}{l}\text { COL IFORM } \\
\text { FECAL } \\
(1100 \mathrm{ML})\end{array}$ & $\begin{array}{l}\text { COL IFORM } \\
\text { TOTAL } \\
(1100 \mathrm{ML})\end{array}$ \\
\hline Wo & $\begin{array}{l}\text { MEAN } \\
\text { MINIMUM } \\
\text { MAXIMUM } \\
\text { NUMBER }\end{array}$ & $\begin{array}{l}11 \\
11 \\
11 \\
1\end{array}$ & $\begin{array}{l}5.0 \\
5.0 \\
5.0 \\
1\end{array}$ & $\begin{array}{l}11.0 \\
11.0 \\
11.0 \\
1\end{array}$ & $\begin{array}{l}6.5 \\
6.5 \\
6.5 \\
1\end{array}$ & $\begin{array}{l}0.01 \\
0.01 \\
0.01 \\
1\end{array}$ & $\begin{array}{l}0.01 \\
0.01 \\
0.01 \\
1\end{array}$ & $\begin{array}{l}51 \\
51 \\
51 \\
1\end{array}$ & $\begin{array}{r}56 \\
56 \\
56 \\
1\end{array}$ & $\begin{array}{l}0 \\
0 \\
0 \\
1\end{array}$ & $\begin{array}{l}0 \\
0 \\
0 \\
1\end{array}$ \\
\hline WP & $\begin{array}{l}\text { MEAN } \\
\text { MINIMUM } \\
\text { MAXIMUM } \\
\text { NUMBER }\end{array}$ & $\begin{array}{l}11 \\
11 \\
11 \\
1\end{array}$ & $\begin{array}{l}4.5 \\
4.5 \\
4.5 \\
1\end{array}$ & $\begin{array}{l}7.8 \\
7.8 \\
7.8 \\
1\end{array}$ & $\begin{array}{l}7.0 \\
7.0 \\
7.0 \\
1\end{array}$ & $\begin{array}{l}0.77 \\
0.77 \\
0.77 \\
1\end{array}$ & $\begin{array}{l}0.01 \\
0.01 \\
0.01 \\
1\end{array}$ & $\begin{array}{r}63 \\
63 \\
63 \\
1\end{array}$ & $\begin{array}{l}67 \\
67 \\
67 \\
1\end{array}$ & $\begin{array}{l}0 \\
0 \\
0 \\
1\end{array}$ & $\begin{array}{l}0 \\
0 \\
0 \\
1\end{array}$ \\
\hline HO & $\begin{array}{l}\text { MEAN } \\
\text { MINIMUM } \\
\text { MAXIMUM } \\
\text { NUMBER }\end{array}$ & $\begin{array}{ll}1 & 1 \\
1 & 1 \\
1 & 1 \\
1\end{array}$ & $\begin{array}{l}4.9 \\
4.9 \\
4.9 \\
1\end{array}$ & $\begin{array}{l}9.7 \\
9.7 \\
9.7 \\
1\end{array}$ & $\begin{array}{l}6.5 \\
6.5 \\
6.5 \\
1\end{array}$ & $\begin{array}{l}0.12 \\
0.12 \\
0.12 \\
1\end{array}$ & $\begin{array}{l}0.01 \\
0.01 \\
0.01 \\
1\end{array}$ & $\begin{array}{r}50 \\
50 \\
50 \\
1\end{array}$ & $\begin{array}{c}52 \\
52 \\
52 \\
1\end{array}$ & $\begin{array}{l}0 \\
0 \\
0 \\
1\end{array}$ & $\begin{array}{l}0 \\
0 \\
0 \\
1\end{array}$ \\
\hline 11 & $\begin{array}{l}\text { MEAN } \\
\text { MINIMUM } \\
\text { MAXIMUM } \\
\text { NUMBER }\end{array}$ & $\begin{array}{r}12 \\
12 \\
12 \\
1\end{array}$ & $\begin{array}{r}5.0 \\
-5.0 \\
5.0 \\
1\end{array}$ & $\begin{array}{l}8.6 \\
8.6 \\
0.6 \\
1\end{array}$ & $\begin{array}{l}10.1 \\
10.1 \\
10.1 \\
1\end{array}$ & $\begin{array}{l}0.01 \\
0.01 \\
0.01 \\
1\end{array}$ & $\begin{array}{l}0.01 \\
0.01 \\
0.01 \\
1\end{array}$ & $\begin{array}{l}41 \\
41 \\
41 \\
1\end{array}$ & $\begin{array}{c}74 \\
74 \\
74 \\
1\end{array}$ & $\begin{array}{l}0 \\
0 \\
0 \\
1\end{array}$ & $\begin{array}{l}0 \\
0 \\
0 \\
1\end{array}$ \\
\hline iJ & $\begin{array}{l}\text { MEAN } \\
\text { MINIMUM } \\
\text { MAXIMUM } \\
\text { NUMBER }\end{array}$ & $\begin{array}{r}15 \\
12 \\
12 \\
12 \\
1\end{array}$ & $\begin{array}{l}5.2 \\
5.2 \\
5.2 \\
1\end{array}$ & $\begin{array}{l}11.0 \\
11.0 \\
11.0 \\
1\end{array}$ & $\begin{array}{l}6.0 \\
6.0 \\
6.0 \\
1\end{array}$ & $\begin{array}{l}0.01 \\
0.01 \\
0.01 \\
1\end{array}$ & $\begin{array}{l}0.01 \\
0.01 \\
0.01 \\
1\end{array}$ & $\begin{array}{r}33 \\
33 \\
33 \\
1\end{array}$ & $\begin{array}{r}48 \\
48 \\
48 \\
1\end{array}$ & $\begin{array}{l}0 \\
0 \\
0 \\
1\end{array}$ & $\begin{array}{l}0 \\
0 \\
0 \\
1\end{array}$ \\
\hline
\end{tabular}

1979 ENL ENVIRONMENTAL MONITORING BUILOING 650 SUMP AREA WELLS WATER CUAL ITY AND PURITY

\begin{tabular}{|c|c|c|c|c|c|c|c|c|c|c|c|}
\hline LOCATION & S SAMPLE & TEMГLRATURE & $\mathrm{PH}$ & $\begin{array}{l}\text { DISSOLVED } \\
\text { OXYGEN } \\
\text { (PPM) }\end{array}$ & $\begin{array}{l}\text { CHLOR IDES } \\
\text { (PPM) }\end{array}$ & $\begin{array}{l}\text { NITRATE } \\
\text { NITROGEN } \\
\text { (PPM) }\end{array}$ & $\begin{array}{l}\text { TOTAL } \\
\text { PHOSPHOROUS } \\
\text { (PPM) }\end{array}$ & $\begin{array}{l}\text { OI SSOL VED } \\
\text { SOL IOS } \\
\text { (PPM) }\end{array}$ & $\begin{array}{l}\text { CONDUC- } \\
\text { TIVITY } \\
\text { MICROMHO/CM }\end{array}$ & $\begin{array}{c}\text { COL IFORM } \\
\text { FECAL } \\
(/ 100 \mathrm{ML})\end{array}$ & $\begin{array}{c}\text { COL IFORM } \\
\text { TOTAL } \\
(1100 \mathrm{ML})\end{array}$ \\
\hline IA & $\begin{array}{l}\text { MEAN } \\
\text { MINIMUM } \\
\text { MAXIMUM } \\
\text { NUMBER }\end{array}$ & $\begin{array}{c}16 \\
15 \\
16 \\
1\end{array}$ & $\begin{array}{l}5.7 \\
5.7 \\
5.7 \\
1\end{array}$ & $\begin{array}{l}6.2 \\
6.2 \\
6.2 \\
1\end{array}$ & $\begin{array}{c}20.8 \\
20.9 \\
20.8 \\
1\end{array}$ & $\begin{array}{l}0.38 \\
0.38 \\
0.38 \\
1\end{array}$ & $\begin{array}{l}0.08 \\
0.08 \\
0.08 \\
1\end{array}$ & $\begin{array}{c}9 \dot{1} \\
02 \\
02 \\
1\end{array}$ & $\begin{array}{r}181 \\
181 \\
181 \\
1\end{array}$ & $\begin{array}{l}0 \\
0 \\
0 \\
1\end{array}$ & $\begin{array}{l}0 \\
0 \\
0 \\
1\end{array}$ \\
\hline $1 C$ & $\begin{array}{l}\text { MEAN } \\
\text { MINIMUM } \\
\text { MAXIMUM } \\
\text { NUMEER }\end{array}$ & $\begin{array}{l}15 \\
15 \\
15 \\
1\end{array}$ & $\begin{array}{l}6.0 \\
6.0 \\
6.0 \\
1\end{array}$ & $\begin{array}{l}7.2 \\
7.2 \\
7.2 \\
1\end{array}$ & $\begin{array}{l}23.0 \\
23.0 \\
23.0 \\
1\end{array}$ & $\begin{array}{l}0.05 \\
0.05 \\
0.05 \\
1\end{array}$ & $\begin{array}{l}0.23 \\
0.23 \\
0.23 \\
1\end{array}$ & $\begin{array}{l}97 \\
97 \\
97 \\
1\end{array}$ & $\begin{array}{r}104 \\
104 \\
104 \\
1\end{array}$ & $\begin{array}{l}0 \\
0 \\
0 \\
1\end{array}$ & $\begin{array}{l}0 \\
0 \\
0 \\
1\end{array}$ \\
\hline 10 & $\begin{array}{l}\text { MF AN } \\
\text { MINIMUM } \\
\text { MAXIMUM } \\
\text { NUMEER }\end{array}$ & $\begin{array}{l}16 \\
16 \\
16 \\
1\end{array}$ & $\begin{array}{l}\text { G.0 } \\
6.0 \\
\text { E.0 } \\
1\end{array}$ & $\begin{array}{l}6 . ? \\
6.2 \\
6.2 \\
1\end{array}$ & $\begin{array}{l}15.8 \\
15.8 \\
15.8 \\
1\end{array}$ & $\begin{array}{l}0.27 \\
0.27 \\
0.27 \\
1\end{array}$ & $\begin{array}{l}0.01 \\
0.01 \\
0.01 \\
1\end{array}$ & $\begin{array}{l}16 \\
15 \\
15 \\
1\end{array}$ & $\begin{array}{l}121 \\
121 \\
123 \\
12 ! \\
1\end{array}$ & $\begin{array}{l}0 \\
0 \\
0 \\
1\end{array}$ & $\begin{array}{l}0 \\
0 \\
0 \\
1\end{array}$ \\
\hline IE & $\begin{array}{l}\text { ME AN } \\
\text { MINIMUM } \\
\text { MAXIMUM } \\
\text { NUMBER }\end{array}$ & $\begin{array}{c}14 \\
14 \\
14 \\
1\end{array}$ & $\begin{array}{l}5.7 \\
5.7 \\
5.7 \\
1\end{array}$ & $\begin{array}{l}5.6 \\
6.6 \\
\text { t. } \\
1\end{array}$ & $\begin{array}{c}14.3 \\
14.3 \\
14.3 \\
1\end{array}$ & $\begin{array}{l}0.69 \\
0.69 \\
0.69 \\
1\end{array}$ & $\begin{array}{l}0.03 \\
0.03 \\
0.03 \\
1\end{array}$ & $\begin{array}{l}14 \\
14 \\
14 \\
1\end{array}$ & $\begin{array}{l}100 \\
100 \\
100 \\
1\end{array}$ & $\begin{array}{l}0 \\
0 \\
0 \\
1\end{array}$ & $\begin{array}{l}0 \\
0 \\
0 \\
1\end{array}$ \\
\hline $1 H$ & $\begin{array}{l}\text { ME AN } \\
\text { MINIMUM } \\
\text { MAXIMUM } \\
\text { PIUMBER }\end{array}$ & $\begin{array}{l}15 \\
15 \\
15 \\
1\end{array}$ & $\begin{array}{l}6.0 \\
6.0 \\
6.0 \\
1\end{array}$ & $\begin{array}{l}5.8 \\
5.8 \\
5.8 \\
1\end{array}$ & $\begin{array}{l}7.7 \\
7.7 \\
7.7 \\
1\end{array}$ & $\begin{array}{l}0.31 \\
0.31 \\
0.31 \\
1\end{array}$ & $\begin{array}{l}0.03 \\
0.03 \\
0.03 \\
1\end{array}$ & $\begin{array}{c}75 \\
75 \\
75 \\
1\end{array}$ & $\begin{array}{r}79 \\
79 \\
79 \\
1\end{array}$ & 0 & 0 \\
\hline
\end{tabular}

Reference Standards - Table 29

Number: samples analyzed per year 
TABLE 24

1970 I'I. ENVIRONMENTAL MONITORING COOL ING WATER WELLS WATT.R QUALITY AND TURITY

\begin{tabular}{|c|c|c|c|c|c|c|c|c|c|c|c|}
\hline LOCATION & N SAMPLE & TEMPERATURE & $\mathrm{PH}$ & $\begin{array}{l}\text { DISSOL VED } \\
\text { OXYOEN } \\
\text { (PPM) }\end{array}$ & $\begin{array}{l}\text { CHLORIOES } \\
\text { (PPM) }\end{array}$ & $\begin{array}{l}\text { NI TRATE } \\
\text { NITROOEN } \\
\text { (PPM) }\end{array}$ & $\begin{array}{c}\text { TOTAL. } \\
\text { PHOSPHOROUS } \\
\text { (PPM) }\end{array}$ & $\begin{array}{l}\text { DISSOL VEO } \\
\text { SOL IDS } \\
\text { (PPM) }\end{array}$ & $\begin{array}{l}\text { D CONOUC- } \\
\text { TIVIITY } \\
\text { MICROMHO/CM }\end{array}$ & $\begin{array}{l}\text { COL IFORM } \\
\text { FECAL } \\
(100 M L)\end{array}$ & $\begin{array}{c}\text { COL IFORM } \\
\text { TOTAL } \\
1 / 100 \mathrm{ML},\end{array}$ \\
\hline$F H$ & $\begin{array}{l}\text { MEAN } \\
\text { MINIMUM } \\
\text { MAXIMUM } \\
\text { NUMEER }\end{array}$ & $\begin{array}{r}13 \\
13 \\
13 \\
2\end{array}$ & $\begin{array}{l}5.8 \\
5.7 \\
5.8 \\
2\end{array}$ & $\begin{array}{l}7.4 \\
7.0 \\
7.8 \\
2\end{array}$ & $\begin{array}{l}17.9 \\
13.5 \\
22.4 \\
2\end{array}$ & $\begin{array}{l}0.10 \\
0.03 \\
0.15 \\
2\end{array}$ & $\begin{array}{l}0.18 \\
0.01 \\
0.36 \\
2\end{array}$ & $\begin{array}{r}93 \\
71 \\
115 \\
2\end{array}$ & $\begin{array}{r}113 \\
88 \\
137 \\
2\end{array}$ & $\begin{array}{l}0 \\
0 \\
0 \\
1\end{array}$ & $\begin{array}{l}0 \\
0 \\
0 \\
1\end{array}$ \\
\hline FI & $\begin{array}{l}\text { MEAN } \\
\text { MINIMUM } \\
\text { MAXIMIIM } \\
\text { NUMBER }\end{array}$ & $\begin{array}{l}11 \\
10 \\
11 \\
3\end{array}$ & $\begin{array}{l}5.8 \\
5.6 \\
6.1 \\
3\end{array}$ & $\begin{array}{l}7.5 \\
7.0 \\
8.2 \\
3\end{array}$ & $\begin{array}{l}12.2 \\
10.7 \\
13.3 \\
3\end{array}$ & $\begin{array}{l}0.02 \\
0.02 \\
0.02 \\
1\end{array}$ & $\begin{array}{l}0.52 \\
0.01 \\
1.28 \\
3\end{array}$ & $\begin{array}{r}64 \\
53 \\
74 \\
3\end{array}$ & $\begin{array}{l}78 \\
73 \\
84 \\
3\end{array}$ & $\begin{array}{l}0 \\
0 \\
0 \\
1\end{array}$ & $\begin{array}{l}\dot{0} \\
0 \\
01 \\
1\end{array}$ \\
\hline FJ & $\begin{array}{l}\text { MEAN } \\
\text { MINIMUM } \\
\text { MAXIMUM } \\
\text { NUMBER }\end{array}$ & $\begin{array}{l}11 \\
10 \\
11 \\
3\end{array}$ & $\begin{array}{l}5.7 \\
5.5 \\
6.0 \\
3\end{array}$ & $\begin{array}{l}4.5 \\
4.0 \\
5.4 \\
3\end{array}$ & $\begin{array}{c}16.9 \\
15.8 \\
18.0 \\
3\end{array}$ & $\begin{array}{l}0.28 \\
0.27 \\
0.31 \\
3\end{array}$ & $\begin{array}{l}0.24 \\
0.03 \\
0.40 \\
3\end{array}$ & $\begin{array}{r}70 \\
66 \\
76 \\
3\end{array}$ & $\begin{array}{r}93 \\
89 \\
96 \\
3\end{array}$ & $\begin{array}{l}0 \\
0 \\
0 \\
?\end{array}$ & $\begin{array}{l}0 \\
0 \\
0 \\
2\end{array}$ \\
\hline$F L$ & $\begin{array}{l}\text { MEAN } \\
\text { MINIMUM } \\
\text { MAXIMUM } \\
\text { NUMELH }\end{array}$ & $\begin{array}{l}14 \\
14 \\
14 \\
3\end{array}$ & $\begin{array}{l}5.7 \\
5.6 \\
5.6 \\
3 .\end{array}$ & $\begin{array}{l}5.9 \\
5.6 \\
7.6 \\
3\end{array}$ & $\begin{array}{c}29.3 \\
28.5 \\
30.2 \\
3\end{array}$ & $\begin{array}{l}1.44 \\
1.41 \\
1.49\end{array}$ & $\begin{array}{l}0.02 \\
0.01 \\
0.04 \\
3\end{array}$ & $\begin{array}{r}123 \\
114 \\
131 \\
8\end{array}$ & $\begin{array}{r}165 \\
1166 \\
1 \% 5 \\
3\end{array}$ & $\begin{array}{l}0 \\
0 \\
0 \\
P\end{array}$ & $\begin{array}{l}0 \\
0 \\
0 \\
?\end{array}$ \\
\hline
\end{tabular}

1979 ENL ENVIROIJMENTAL MONITORING TAP WATER WATER QUALITY AND PURI IY

\begin{tabular}{|c|c|c|c|c|c|c|c|c|c|c|c|}
\hline LOCATION & V SAMPLE & TEMPERA TURE & $\mathrm{PH}$ & $\begin{array}{l}\text { DISSOL VED } \\
\text { OXYGEN } \\
\text { (PPM) }\end{array}$ & $\underset{(P P M)}{\text { CHLORIOES }}$ & $\begin{array}{l}\text { NITRATE } \\
\text { NITROGEN } \\
\text { (PPM) }\end{array}$ & $\begin{array}{l}\text { TOTAL } \\
\text { PHOSPHOROUS } \\
\text { (PPM) }\end{array}$ & $\begin{array}{l}\text { DISSOL VEO } \\
\text { SOL IOS } \\
\text { (PPM) }\end{array}$ & $\begin{array}{l}\text { CONDUC- } \\
\text { TIVITY } \\
\text { MICRUMHU/CM }\end{array}$ & $\begin{array}{c}\text { COL IFORM } \\
\text { FECAL } \\
(/ 100 \mathrm{ML})\end{array}$ & $\begin{array}{c}\text { COL IFORM } \\
\text { TOTAL } \\
(100 \mathrm{ML})\end{array}$ \\
\hline FN & $\begin{array}{l}\text { MEAN } \\
\text { MINIMUM } \\
\text { MAXIMUM } \\
\text { NUMBER }\end{array}$ & 0 & $\begin{array}{l}5.3 \\
5.3 \\
5.3 \\
1\end{array}$ & 0 & $\begin{array}{l}19.9 . \\
16.7 \\
24.5 \\
53\end{array}$ & $\begin{array}{l}0.42 \\
0.03 \\
3.34 \\
53\end{array}$ & $\begin{array}{l}0.05 \\
0.01 \\
0.25 \\
52\end{array}$ & $\begin{array}{r}88 \\
71 \\
126 \\
12\end{array}$ & 0 & 0 & 0 \\
\hline
\end{tabular}

1979 BNL ENVIRONMENTAL MONITORING POTABLE SUPPLY WELLS WATER OUAL ITY AND PURITY

\begin{tabular}{|c|c|c|c|c|c|c|c|c|c|c|c|}
\hline LOCATION & $\checkmark$ SAMPLE & TEMPERATURE & $\mathrm{PH}$ & $\begin{array}{l}\text { DISSOLVED } \\
\text { OXYGEN } \\
\text { (PPM) }\end{array}$ & $\begin{array}{l}\text { CHLUKIUES } \\
\text { (PPM) }\end{array}$ & $\begin{array}{l}\text { NITRATE } \\
\text { NI I I HUGEEIN } \\
\text { (PPMI) }\end{array}$ & $\begin{array}{c}\text { TOTAL } \\
\text { PHiUSPHAROUS } \\
\text { (PPM) }\end{array}$ & $\begin{array}{l}\text { OISSOLVEO } \\
\text { 9OL } 109 \\
\text { (PPM) }\end{array}$ & $\begin{array}{l}\text { CONOUC- } \\
\text { TIVITY } \\
\text { MICROMHO/CM }\end{array}$ & $\begin{array}{l}\text { COL IFORM } \\
\text { FECAL } \\
(1100 \mathrm{ML})\end{array}$ & $\begin{array}{c}\text { COL IFORM } \\
\text { TOTAL } \\
(100 \mathrm{ML},\end{array}$ \\
\hline FB & $\begin{array}{l}\text { ME AN } \\
\text { MINIMIIM } \\
\text { MAXIMUM } \\
\text { NUMBER }\end{array}$ & $\begin{array}{r}1.3 \\
12 \\
13 \\
4\end{array}$ & $\begin{array}{l}5.9 \\
5.7 \\
5.0 \\
4\end{array}$ & $\begin{array}{l}7.0 \\
6: 4 \\
7.4 \\
4\end{array}$ & $\begin{array}{l}21.7 \\
18.5 \\
28.6 \\
4\end{array}$ & $\begin{array}{l}0.73 \\
0.5 \% \\
0.91 \\
4\end{array}$ & $\begin{array}{l}0.04 \\
4.01 \\
0.08 \\
4\end{array}$ & $\begin{array}{r}112 \\
92 \\
148 \\
3\end{array}$ & $\begin{array}{r}134 \\
128 \\
102 \\
102 \\
4\end{array}$ & $\begin{array}{l}0 \\
0 \\
0 \\
4\end{array}$ & $\begin{array}{l}0 \\
0 \\
0 \\
4\end{array}$ \\
\hline FD & $\begin{array}{l}\text { MEAN } \\
\text { MINIMUM } \\
\text { MAXIMUM } \\
\text { NUMBER }\end{array}$ & $\begin{array}{r}11 \\
10 \\
12 \\
4\end{array}$ & $\begin{array}{l}5.8 \\
5.7 \\
5.0 \\
4\end{array}$ & $\begin{array}{l}7.7 \\
7.0 \\
8.0 \\
4\end{array}$ & $\begin{array}{c}17.8 \\
16.8 \\
19.3 \\
4\end{array}$ & $\begin{array}{l}0.25 . \\
0.11 \\
0.40 \\
4\end{array}$ & $\begin{array}{l}0.04 \\
0.01 \\
0.06 \\
4\end{array}$ & $\begin{array}{r}71 \\
69 \\
74 \\
3\end{array}$ & $\begin{array}{r}97 \\
91 \\
104 \\
4\end{array}$ & $\begin{array}{l}0 \\
0 \\
0 \\
4\end{array}$ & $\begin{array}{l}0 \\
0 \\
0 \\
4\end{array}$ \\
\hline FE & $\begin{array}{l}\text { MEAN } \\
\text { MINIMUM } \\
\text { MAXIMUM } \\
\text { NUMBER }\end{array}$ & $\begin{array}{r}13 \\
10 \\
19 \\
3\end{array}$ & $\begin{array}{l}5.8 \\
5.4 \\
6.1 \\
4\end{array}$ & $\begin{array}{r}8.3 \\
4.0 \\
10.8 \\
14\end{array}$ & $\begin{array}{l}5.2 \\
5.4 \\
7.7 \\
4\end{array}$ & $\begin{array}{l}0.08 \\
0.01 \\
0.23 \\
4\end{array}$ & $\begin{array}{l}0.03 \\
0.01 \\
0.05 \\
4\end{array}$ & $\begin{array}{r}30 \\
35 \\
42 \\
3\end{array}$ & $\begin{array}{r}74 \\
46 \\
154 \\
4\end{array}$ & $\begin{array}{l}0 \\
0 \\
0 \\
4\end{array}$ & $\begin{array}{l}0 \\
0 \\
0 \\
4\end{array}$ \\
\hline FF & $\begin{array}{l}\text { MEAN } \\
\text { MINIMUM } \\
\text { MAXIMUM } \\
\text { NUMBER }\end{array}$ & $\begin{array}{l}12 \\
11 \\
12 \\
4\end{array}$ & $\begin{array}{l}5.7 \\
5.5 \\
6 \text { n } \\
4\end{array}$ & $\begin{array}{l}\text { 9.6 } \\
8.6 \\
8.5 \\
4\end{array}$ & $\begin{array}{r}17.5 \\
6.6 \\
2.3 .0 \\
4\end{array}$ & $\begin{array}{l}0.50 \\
0.37 \\
0.50 \\
4\end{array}$ & $\begin{array}{l}0.03 \\
0.01 \\
0.05 \\
4\end{array}$ & $\begin{array}{r}68 \\
80 \\
90 \\
1\end{array}$ & $\begin{array}{r}118 \\
114 \\
124 \\
4\end{array}$ & $\begin{array}{l}0 \\
0 \\
0 \\
7\end{array}$ & $\begin{array}{l}0 \\
0 \\
0 \\
3\end{array}$ \\
\hline$F G$ & $\begin{array}{l}\text { MEAN } \\
\text { MINIMUM } \\
\text { MAXIMUM } \\
\text { NUMGER }\end{array}$ & $\begin{array}{l}10 \\
10 \\
11 \\
3\end{array}$ & $\begin{array}{l}5.9 \\
5.5 \\
6.2 \\
3\end{array}$ & $\begin{array}{l}8.2 \\
8.0 \\
8.6 \\
3\end{array}$ & $\begin{array}{c}11.4 \\
9.5 \\
13.3 \\
3\end{array}$ & $\begin{array}{l}0.05 \\
0.01 \\
0.08 \\
3\end{array}$ & $\begin{array}{l}0.03 \\
0.01 \\
0.05 \\
3\end{array}$ & $\begin{array}{r}59 \\
50 \\
80 \\
2\end{array}$ & $\begin{array}{r}71 \\
69 \\
74 \\
3\end{array}$ & $\begin{array}{l}0 \\
0 \\
0 \\
3\end{array}$ & $\begin{array}{l}0 \\
0 \\
0 \\
3\end{array}$ \\
\hline
\end{tabular}

Reference Standards - Table 29

Locations Indicated on Fig. 8 \& identified as: Potable - FA: 1, FB: 2, FC: 3, FD: 4, FE: 5, Number: samples analyzed per year $\mathrm{FF}: 6, \mathrm{FG}: 7$ Cooling - FH: 101, FI: 102, FJ: 103, FK: 1U4, FL: 105 . 
TABLE 25

1979 BNL ENYIRONMENTAL MONITORING SAND FILTER BEDS AND PECONIC RIVER AREA WELLS WATER OUALITY AND PURITY-METALS

\begin{tabular}{|c|c|c|c|c|c|c|c|c|}
\hline & & \multicolumn{7}{|c|}{ METALS (IN PPM) } \\
\hline$X A$ & $\begin{array}{l}\text { MEAN } \\
\text { MINIMUM } \\
\text { MAXIMUM } \\
\text { EXCEPT ION } \\
\text { NUMBER }\end{array}$ & $\begin{array}{l}.001 \\
.001 \\
.001 \\
4 \\
4\end{array}$ & $\begin{array}{l}.0009 \\
.0009 \\
.0010 \\
0 \\
4\end{array}$ & $\begin{array}{l}.015 \\
.002 \\
.054 \\
3 \\
4\end{array}$ & $\begin{array}{l}.007 \\
.005 \\
.009 \\
0 \\
4\end{array}$ & $\begin{array}{l}.516 \\
.110 \\
1.650 \\
0 \\
4\end{array}$ & $\begin{array}{l}.004 \\
.002 \\
.005 \\
4 \\
4\end{array}$ & $\begin{array}{l}.284 \\
.262 \\
.319 \\
0 \\
3\end{array}$ \\
\hline XB & $\begin{array}{l}\text { MEAN } \\
\text { MINIMUM } \\
\text { MAXIMUM } \\
\text { EXCEPT ION } \\
\text { NUMBER }\end{array}$ & $\begin{array}{l}.00 ! \\
.00 ! \\
i \\
i\end{array}$ & $\begin{array}{l}.0003 \\
.0003 \\
i^{0003}\end{array}$ & $\begin{array}{l}.001 \\
.001 \\
i^{001}\end{array}$ & $\begin{array}{l}.003 \\
.003 \\
.003 \\
0 \\
1\end{array}$ & $\begin{array}{l}.200 \\
.200 \\
.200 \\
0 \\
1\end{array}$ & $\begin{array}{l}.011 \\
.011 \\
.011 \\
0 \\
1\end{array}$ & $\begin{array}{c}3.930 \\
3.930 \\
3.930 \\
0 \\
1\end{array}$ \\
\hline$x C$ & $\begin{array}{l}\text { MEAN } \\
\text { MINIMUM } \\
\text { MAXIMUM } \\
\text { EXCEPTION } \\
\text { NUMBER }\end{array}$ & $\begin{array}{l}.001 \\
.001 \\
i^{001} \\
1\end{array}$ & $\begin{array}{l}.0003 \\
.0003 \\
i^{0003} \\
1\end{array}$ & $\begin{array}{l}.001 \\
.001 \\
i^{001} \\
1\end{array}$ & $\begin{array}{l}.007 \\
.007 \\
.007 \\
0 \\
1\end{array}$ & $\begin{array}{l}1.610 \\
1.610 \\
1.610 \\
0 \\
1\end{array}$ & $\begin{array}{l}.002 \\
.002 \\
.002 \\
1 \\
1\end{array}$ & $\begin{array}{l}.610 \\
.610 \\
.610 \\
0 \\
1\end{array}$ \\
\hline XD & $\begin{array}{l}\text { MEAN } \\
\text { MINIMUM } \\
\text { MAXIMUM } \\
\text { EXCEPTION } \\
\text { NUMBER }\end{array}$ & $\begin{array}{l}.001 \\
.001 \\
.001 \\
i\end{array}$ & $\begin{array}{l}.0003 \\
.0003 \\
i .0003 \\
i\end{array}$ & $\begin{array}{l}.023 \\
.023 \\
.023 \\
0 \\
1\end{array}$ & $\begin{array}{l}.003 \\
.003 \\
.003 \\
0 \\
1\end{array}$ & $\begin{array}{l}.720 \\
.720 \\
.720 \\
0 \\
1\end{array}$ & $\begin{array}{l}.006 \\
.005 \\
.006 \\
0 \\
1\end{array}$ & $\begin{array}{l}.204 \\
.204 \\
.204 \\
0 \\
1\end{array}$ \\
\hline$X E$ & $\begin{array}{l}\text { MEAN } \\
\text { MINIMUM } \\
\text { MAXIMUM } \\
\text { EXCEPTION } \\
\text { NUMBER }\end{array}$ & $\begin{array}{l}.001 \\
.001 \\
i^{001}\end{array}$ & $\begin{array}{l}0 \\
0\end{array}$ & $\begin{array}{l}.002 \\
.002 \\
.002 \\
i\end{array}$ & $\begin{array}{l}.006 \\
.006 \\
.006 \\
0 \\
1\end{array}$ & $\begin{array}{l}.450 \\
.450 \\
.450 \\
0 \\
1\end{array}$ & $\begin{array}{l}.002 \\
.002 \\
.002 \\
0 \\
1\end{array}$ & $\begin{array}{l}.953 \\
.953 \\
.953 \\
0 \\
1\end{array}$ \\
\hline XF & $\begin{array}{l}\text { MEAN } \\
\text { MINIMUM } \\
\text { MAXIMUM } \\
\text { EXCEPIION } \\
\text { NUMBER }\end{array}$ & $\begin{array}{l}.001 \\
.001 \\
i^{001}\end{array}$ & $\begin{array}{l}0 \\
0\end{array}$ & $\begin{array}{l}.005 \\
.005 \\
.005 \\
0 \\
1\end{array}$ & $\begin{array}{l}.009 \\
.009 \\
.009 \\
0 \\
1\end{array}$ & $\begin{array}{l}.083 \\
.083 \\
.083 \\
0 \\
1\end{array}$ & $\begin{array}{l}.027 \\
.027 \\
.027 \\
0 \\
1\end{array}$ & $\begin{array}{l}2.430 \\
2.430 \\
2.430 \\
0 \\
1\end{array}$ \\
\hline$X G$ & $\begin{array}{l}\text { MEAN } \\
\text { MINIMUM } \\
\text { MAXIMUM } \\
\text { EXCEPT ION } \\
\text { NUMBER }\end{array}$ & $\begin{array}{l}.001 \\
.001 \\
.001 \\
5 \\
5\end{array}$ & $\begin{array}{l}.0005 \\
.0002 \\
.0006 \\
5 \\
5\end{array}$ & $\begin{array}{l}.002 \\
.001 \\
.002 \\
5 \\
5\end{array}$ & $\begin{array}{l}.005 \\
.003 \\
.013 \\
0 \\
5\end{array}$ & $\begin{array}{c}3.328 \\
1.190 \\
6.600 \\
0 \\
b\end{array}$ & $\begin{array}{l}.004 \\
.002 \\
.005 \\
5 \\
5\end{array}$ & $\begin{array}{l}.094 \\
.064 \\
.126 \\
0 \\
5\end{array}$ \\
\hline$X H$ & $\begin{array}{l}\text { MEAN } \\
\text { MINIMUM } \\
\text { MAXIMUM } \\
\text { EXCEPT ION } \\
\text { NUMBER }\end{array}$ & $\begin{array}{l}.001 \\
.001 \\
i .001 \\
i\end{array}$ & $\begin{array}{l}.0005 \\
.0005 \\
.0005 \\
0 \\
1\end{array}$ & $\begin{array}{l}.001 \\
.001 \\
i^{001} \\
i\end{array}$ & $\begin{array}{l}.005 \\
.005 \\
.005 \\
0 \\
1\end{array}$ & $\begin{array}{c}3.750 \\
3.750 \\
3.750 \\
0 \\
1\end{array}$ & $\begin{array}{l}.005 \\
.005 \\
.005 \\
0 \\
1\end{array}$ & $\begin{array}{l}.823 \\
.823 \\
.823 \\
0 \\
1\end{array}$ \\
\hline$X I$ & $\begin{array}{l}\text { MEAN } \\
\text { MINIMUM } \\
\text { MAXIMUM } \\
\text { EXCEPI ION } \\
\text { NUMBER }\end{array}$ & $\begin{array}{l}.001 \\
.001 \\
.001 \\
i\end{array}$ & $\begin{array}{l}.0003 \\
.0003 \\
.0003 \\
i\end{array}$ & $\begin{array}{l}.015 \\
.015 \\
.015 \\
0 \\
1\end{array}$ & $\begin{array}{l}.005 \\
.005 \\
.005 \\
0 \\
1\end{array}$ & $\begin{array}{l}100 \\
.100 \\
100 \\
0 \\
1\end{array}$ & $\begin{array}{l}.011 \\
.011 \\
.011 \\
0 \\
1\end{array}$ & $\begin{array}{l}.460 \\
.460 \\
.460 \\
0 \\
1\end{array}$ \\
\hline XJ & $\begin{array}{l}\text { MEAN } \\
\text { MINIMUM } \\
\text { MAXIMUM } \\
\text { EXCEPT ION } \\
\text { NUMBER }\end{array}$ & $\begin{array}{l}.001 \\
.001 \\
i^{001} \\
i\end{array}$ & $\begin{array}{l}.0002 \\
.0002 \\
i^{.0002} \\
i\end{array}$ & $\begin{array}{l}.002 \\
.002 \\
.002 \\
i\end{array}$ & $\begin{array}{l}.003 \\
.003 \\
.003 \\
0 \\
1\end{array}$ & $\begin{array}{l}1.100 \\
1.100 \\
1.100 \\
0 \\
1\end{array}$ & $\begin{array}{l}.002 \\
.002 \\
i^{002}\end{array}$ & $\begin{array}{l}.511 \\
.511 \\
.511 \\
0 \\
1\end{array}$ \\
\hline $\mathrm{XK}$ & $\begin{array}{l}\text { MEAN } \\
\text { MINIMUM } \\
\text { MAXIMUM } \\
\text { EXCEPTION } \\
\text { NUMBER }\end{array}$ & $\begin{array}{l}.001 \\
.001 \\
.001 \\
5 \\
5\end{array}$ & $\begin{array}{l}.0005 \\
.0005 \\
.0009 \\
5 \\
5\end{array}$ & $\begin{array}{l}.002 \\
.002 \\
.003 \\
3 \\
5\end{array}$ & $\begin{array}{l}.003 \\
.002 \\
i^{004}\end{array}$ & $\begin{array}{l}2.004 \\
1.510 \\
2.480 \\
0 \\
5\end{array}$ & $\begin{array}{l}.004 \\
.002 \\
.005 \\
5 \\
5\end{array}$ & $\begin{array}{l}1.119 \\
.363 \\
2.320 \\
0 \\
5\end{array}$ \\
\hline$X L$ & $\begin{array}{l}\text { MEAN } \\
\text { MINIMUM } \\
\text { MAXIMUM } \\
\text { EXCEPT ION } \\
\text { NUMBER }\end{array}$ & $\begin{array}{l}.001 \\
.001 \\
.001 \\
4 \\
4\end{array}$ & $\begin{array}{l}.0005 \\
.0003 \\
.0006 \\
4^{4}\end{array}$ & $\begin{array}{l}.002 \\
.002 \\
.002 \\
3 \\
4\end{array}$ & $\begin{array}{l}.003 \\
.002 \\
.004 \\
4\end{array}$ & $\begin{array}{l}2.888 \\
1.650 \\
5.890 \\
0 \\
4\end{array}$ & $\begin{array}{l}.004 \\
.002 \\
.005 \\
3 \\
4\end{array}$ & $\begin{array}{l}.577 \\
.288 \\
.840 \\
0 \\
4\end{array}$ \\
\hline$X M$ & $\begin{array}{l}\text { MEAN } \\
\text { MINIMUM } \\
\text { MAXIMUM } \\
\text { EXCEPT ION } \\
\text { NUMEER }\end{array}$ & $\begin{array}{l}.001 \\
.001 \\
.001 \\
3 \\
3\end{array}$ & $\begin{array}{l}.0007 \\
.0006 \\
.0010 \\
4 \\
4\end{array}$ & $\begin{array}{l}.003 \\
.002 \\
.005 \\
3 \\
4\end{array}$ & $\begin{array}{l}.015 \\
.011 \\
.016 \\
0 \\
4\end{array}$ & $\begin{array}{c}3.530 \\
2.310 \\
5.520 \\
0 \\
4\end{array}$ & $\begin{array}{l}.007 \\
.005 \\
.011 \\
3 \\
4\end{array}$ & $\begin{array}{c}.935 \\
.718 \\
1,160 \\
0 \\
4\end{array}$ \\
\hline$X P Y$ & $\begin{array}{l}\text { MCANA } \\
\text { MINIMUM } \\
\text { MAXIMUM } \\
\text { EXCEPTION } \\
\text { NUMBER }\end{array}$ & $\begin{array}{l}.001 \\
.001 \\
.001 \\
i\end{array}$ & $\begin{array}{l}.0002 \\
.0002 \\
i \\
i\end{array}$ & $\begin{array}{l}.003 \\
.003 \\
.003 \\
0 \\
1\end{array}$ & $\begin{array}{l}.008 \\
.009 \\
.009 \\
0 \\
1\end{array}$ & $\begin{array}{l}8.050 \\
9.050 \\
9.050 \\
0 \\
1\end{array}$ & $\begin{array}{l}.010 \\
.010 \\
.010 \\
0 \\
1\end{array}$ & $\begin{array}{l}1.220 \\
1.220 \\
1.220 \\
0 \\
1\end{array}$ \\
\hline$x 0$ & $\begin{array}{l}\text { MEAN } \\
\text { MINIMUM } \\
\text { MAXIMUM } \\
\text { EXCEPT ION } \\
\text { NUMBER }\end{array}$ & $\begin{array}{l}.001 \\
.001 \\
.001 \\
e^{2}\end{array}$ & $\begin{array}{l}.0032 \\
.0003 \\
.0080 \\
e^{2}\end{array}$ & $\begin{array}{l}.003 \\
.002 \\
.003 \\
i^{2}\end{array}$ & $\begin{array}{l}.003 \\
.003 \\
.003 \\
0 \\
1\end{array}$ & $\begin{array}{r}15.100 \\
6.600 \\
23.600 \\
0 \\
2\end{array}$ & $\begin{array}{l}.122 \\
.005 \\
i^{238} \\
i^{1}\end{array}$ & $\begin{array}{l}.237 \\
.215 \\
.260 \\
0 \\
5\end{array}$ \\
\hline
\end{tabular}


TABLE 25 (Continued)

1979 BNL ENYIRONMENTAL MONITORING LANDFILL AREA WELLS WATER QLAL ! TY AND PUR ! TY-METALS

\begin{tabular}{|c|c|c|c|c|c|c|c|c|}
\hline & & \multicolumn{6}{|c|}{ METALS (IN PPM) } & $\mathrm{ZN}$ \\
\hline XR & $\begin{array}{l}\text { MEAN } \\
\text { MINIMUM } \\
\text { MAXIMUM } \\
\text { EXCEPTION } \\
\text { NUMBER }\end{array}$ & $\begin{array}{l}.001 \\
.001 \\
i^{001}\end{array}$ & $\begin{array}{l}.0003 \\
.0003 \\
.0003 \\
i\end{array}$ & $\begin{array}{l}.001 \\
.001 \\
.001 \\
i\end{array}$ & $\begin{array}{l}.006 \\
.006 \\
.006 \\
0 \\
1\end{array}$ & $\begin{array}{l}.020 \\
.020 \\
.020 \\
0 \\
1\end{array}$ & $\begin{array}{l}.009 \\
.009 \\
.009 \\
i\end{array}$ & $\begin{array}{l}1.210 \\
1.210 \\
1.210 \\
0\end{array}$ \\
\hline$x 5$ & $\begin{array}{l}\text { MEAN } \\
\text { MINIMUUM } \\
\text { MAXIMUM } \\
\text { EXCEPTION } \\
\text { NUMBER }\end{array}$ & $\begin{array}{l}.002 \\
.001 \\
.007 \\
4 \\
4\end{array}$ & $\begin{array}{l}.0004 \\
.0002 \\
.0006 \\
4 \\
4\end{array}$ & $\begin{array}{l}.002 \\
.001 \\
.002 \\
4 \\
4\end{array}$ & $\begin{array}{l}.016 \\
.014 \\
.018 \\
0 \\
4\end{array}$ & $\begin{array}{l}3.958 \\
3.210 \\
4.500 \\
0 \\
4\end{array}$ & $\begin{array}{l}.004 \\
.002 \\
.005 \\
4 \\
4\end{array}$ & $\begin{array}{l}.143 \\
.126 \\
.169 \\
0^{169}\end{array}$ \\
\hline$X W$ & $\begin{array}{l}\text { MEAN } \\
\text { MINIMUM } \\
\text { MAXIMUM } \\
\text { EXCEPTION } \\
\text { NUMBER }\end{array}$ & $\begin{array}{l}.001 \\
.001 \\
i^{001}\end{array}$ & $\begin{array}{l}.0003 \\
.0003 \\
.0003 \\
i\end{array}$ & $\begin{array}{l}.001 \\
.001 \\
i 001 \\
i\end{array}$ & $\begin{array}{l}.005 \\
.005 \\
.005 \\
0 \\
1\end{array}$ & $\begin{array}{l}.560 \\
.560 \\
.560 \\
0 \\
1\end{array}$ & $\begin{array}{l}.002 \\
.002 \\
i .002 \\
i\end{array}$ & $\begin{array}{l}192 \\
.192 \\
192 \\
0^{192} \\
1\end{array}$ \\
\hline \multirow[t]{2}{*}{$x x$} & MEAN & .001 & .0005 & .002 & .003 & 5. & i . ēót & .184 \\
\hline & $\begin{array}{l}\text { MINIMUM } \\
\text { MAX!MUM } \\
\text { EXCEPT I ONI } \\
\text { MUMBER }\end{array}$ & $\begin{array}{l}.001 \\
.001 \\
5 \\
5\end{array}$ & $\begin{array}{l}.0002 \\
.0006 \\
5 \\
5\end{array}$ & $\begin{array}{l}.002 \\
.002 \\
4 \\
5\end{array}$ & $\begin{array}{l}.002 \\
.003 \\
0 \\
5\end{array}$ & $\begin{array}{c}4.530 \\
6.410 \\
0 \\
5\end{array}$ & $\begin{array}{l}.002 \\
6.005 \\
3 \\
5\end{array}$ & $\begin{array}{l}.133 \\
.230 \\
0 \\
b\end{array}$ \\
\hline$X Y$ & $\begin{array}{l}\text { MEAN } \\
\text { MIINIMUM } \\
\text { MAXIMUM } \\
\text { EXCEPT ION } \\
\text { NUMBCR }\end{array}$ & $\begin{array}{l}.001 \\
.001 \\
.001 \\
i\end{array}$ & $\begin{array}{l}.0003 \\
.0003 \\
.0003 \\
0^{1}\end{array}$ & $\begin{array}{l}.002 \\
.002 \\
i^{002} \\
i\end{array}$ & $\begin{array}{l}.004 \\
.004 \\
.004 \\
0 \\
1\end{array}$ & $\begin{array}{l}281 \\
.281 \\
.281 \\
0 \\
1\end{array}$ & $\begin{array}{l}.007 \\
.007 \\
.007 \\
0 \\
1\end{array}$ & $\begin{array}{l}.600 \\
.600 \\
.600 \\
0 \\
1\end{array}$ \\
\hline$x z$ & $\begin{array}{l}\text { MEAN } \\
\text { MINIMUM } \\
\text { MAXIMIIM } \\
\text { EXCEPT ION } \\
\text { NUMBER }\end{array}$ & $\begin{array}{l}.001 \\
.001 \\
i_{1}^{001}\end{array}$ & $\begin{array}{l}.0003 \\
.0003 \\
i^{.0003} \\
1\end{array}$ & $\begin{array}{l}.001 \\
.001 \\
.001 \\
i \\
i\end{array}$ & $\begin{array}{l}.003 \\
.003 \\
.003 \\
0 \\
1\end{array}$ & $\begin{array}{l}.010 \\
.010 \\
.010 \\
\mathrm{U}^{\mathrm{i}}\end{array}$ & $\begin{array}{l}.002 \\
.002 \\
.002 \\
i\end{array}$ & $\begin{array}{l}.236 \\
.236 \\
.236 \\
0 \\
1\end{array}$ \\
\hline$x 1$ & $\begin{array}{l}\text { MEAN } \\
\text { MINIMUM } \\
\text { MAXIMUM } \\
\text { EXCEPTION } \\
\text { NUMEER }\end{array}$ & $\begin{array}{l}.001 \\
.001 \\
.001 \\
i\end{array}$ & $\begin{array}{l}.0003 \\
.0003 \\
i^{0003}\end{array}$ & $\begin{array}{l}.002 \\
.002 \\
.002 \\
0 \\
1\end{array}$ & $\begin{array}{l}.004 \\
.004 \\
.004 \\
0 \\
1\end{array}$ & $\begin{array}{l}.020 \\
.020 \\
.020 \\
0 \\
1\end{array}$ & $\begin{array}{l}.002 \\
.002 \\
i^{002}\end{array}$ & $\begin{array}{l}.973 \\
.973 \\
.973 \\
0 \\
1\end{array}$ \\
\hline xa & $\begin{array}{l}\text { MEANI } \\
\text { MIN!MUM } \\
\text { MAXIMUM } \\
\text { EXCEPT ION } \\
\text { NUMBER }\end{array}$ & $\begin{array}{l}n n 1 \\
.001 \\
i^{001}\end{array}$ & $\begin{array}{l}.0 n n ? \\
.0002 \\
.0002 \\
i\end{array}$ & $\begin{array}{l}.005 \\
.005 \\
.005 \\
0 \\
i\end{array}$ & $\begin{array}{l}.003 \\
.003 \\
.003 \\
0 \\
1\end{array}$ & $\begin{array}{l}.050 \\
.050 \\
.050 \\
0 \\
1\end{array}$ & $\begin{array}{l}.002 \\
.002 \\
.002 \\
i \\
1\end{array}$ & $\begin{array}{l}1.450 \\
1.450 \\
1.450 \\
0 \\
1\end{array}$ \\
\hline wr & $\begin{array}{l}\text { MEAN } \\
\text { MIINIPUUM } \\
\text { MAXIMUM } \\
\text { EXCEPT I ON } \\
\text { NUMBER }\end{array}$ & $\begin{array}{l}.001 \\
.001 \\
.001 \\
2^{2}\end{array}$ & $\begin{array}{l}.0004 \\
.0002 \\
.0006 \\
e^{2}\end{array}$ & $\begin{array}{l}. n n n \\
.000 \\
.000 \\
2^{2}\end{array}$ & $\begin{array}{l}.005 \\
\operatorname{lnz}_{008} \\
.008 \\
\stackrel{0}{2}\end{array}$ & $\begin{array}{l}.452 \\
.226 \\
.579 \\
0 \\
2\end{array}$ & $\begin{array}{l}.006 \\
.005 \\
.007 \\
i^{2}\end{array}$ & $\begin{array}{l}.407 \\
.399 \\
.414 \\
0 \\
2\end{array}$ \\
\hline WG & $\begin{array}{l}\text { MEAN } \\
\text { MINIMUM } \\
\text { MAXIMUM } \\
\text { EXCEPTION } \\
\text { NUMBEF }\end{array}$ & $\begin{array}{l}.001 \\
.001 \\
.001 \\
e^{2}\end{array}$ & $\begin{array}{l}.0004 \\
.0002 \\
.0006 \\
e^{2}\end{array}$ & $\begin{array}{l}.002 \\
.002 \\
.002 \\
2 \\
2\end{array}$ & $\begin{array}{l}.004 \\
.003 \\
.005 \\
0 \\
3\end{array}$ & $\begin{array}{l}8.200 \\
7.420 \\
8.980 \\
0 \\
a\end{array}$ & $\begin{array}{l}.004 \\
.002 \\
.005 \\
2^{2}\end{array}$ & $\begin{array}{l}.169 \\
.139 \\
199 \\
0 \\
?\end{array}$ \\
\hline WR & $\begin{array}{l}\text { MEAN } \\
\text { MIPNIMUM } \\
\text { MAXIHUM } \\
\text { EXCEPT ION } \\
\text { IUUI IDER }\end{array}$ & $\begin{array}{l}.003 \\
.003 \\
.003 \\
1\end{array}$ & $\begin{array}{l}.0006 \\
. \text { UUUb } \\
.0006 \\
i\end{array}$ & 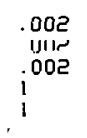 & $\begin{array}{l}.007 \\
.00107 \\
.0107 \\
0 \\
1\end{array}$ & $\begin{array}{c}4 E .230 \\
4 \pi .830 \\
45.230 \\
0 \\
1\end{array}$ & $\begin{array}{l}.005 \\
.005 \\
.005 \\
i\end{array}$ & $\begin{array}{l}103 \\
103 \\
103 \\
0 \\
1\end{array}$ \\
\hline WS & $\begin{array}{l}\text { MEAN } \\
\text { MIN! MUM } \\
\text { MAXIMUPI } \\
\text { EXCEPT ION } \\
\text { MIIMEER }\end{array}$ & $\begin{array}{l}.001 \\
.001 \\
.001 \\
1 \\
1\end{array}$ & $\begin{array}{l}.0006 \\
.0006 \\
i^{0006}\end{array}$ & $\begin{array}{l}.002 \\
.002 \\
.002 \\
0 \\
1\end{array}$ & $\begin{array}{l}.005 \\
.005 \\
0.05 \\
0 \\
1\end{array}$ & $\begin{array}{c}\text { 2ट. } 600 \\
22.600 \\
22.600 \\
0 \\
1\end{array}$ & $\begin{array}{l}.007 \\
.007 \\
.007 \\
1\end{array}$ & $\begin{array}{l}.358 \\
.358 \\
.358 \\
0 \\
1\end{array}$ \\
\hline WT & $\begin{array}{l}\text { MEAN } \\
\text { MINIMUM } \\
\text { MAXIMUM } \\
\text { EXCEPTION } \\
\text { NUMBER }\end{array}$ & $\begin{array}{l}.001 \\
.001 \\
.001 \\
2 \\
2\end{array}$ & $\begin{array}{l}.0005 \\
.0003 \\
i^{0005} \\
i^{0}\end{array}$ & $\begin{array}{l}.002 \\
.002 \\
.002 \\
5\end{array}$ & $\begin{array}{l}.004 \\
.002 \\
.006 \\
0 \\
2\end{array}$ & $\begin{array}{l}.797 \\
.717 \\
.876 \\
0 \\
2\end{array}$ & $\begin{array}{l}.004 \\
.003 \\
.005 \\
i^{2}\end{array}$ & $\begin{array}{l}2.495 \\
2.320 \\
2.670 \\
0 \\
2\end{array}$ \\
\hline W9 & $\begin{array}{l}\text { MEAN } \\
\text { MINIMUM } \\
\text { MAXIMUIH } \\
\text { EXCEPTION } \\
\text { NUMBER }\end{array}$ & $\begin{array}{l}.002 \\
.001 \\
j^{004} \\
2\end{array}$ & $\begin{array}{l}.0004 \\
.0002 \\
.0006 \\
e^{2}\end{array}$ & $\begin{array}{l}.004 \\
.002 \\
.005 \\
i^{2}\end{array}$ & $\begin{array}{l}.006 \\
.006 \\
.006 \\
0 \\
1\end{array}$ & $\begin{array}{c}38.300 \\
19.300 \\
57300 \\
0 \\
2\end{array}$ & $\begin{array}{l}.004 \\
.002 \\
.106 \\
i^{n 06}\end{array}$ & $\begin{array}{l}.192 \\
.053 \\
.321 \\
0^{2}\end{array}$ \\
\hline IK & $\begin{array}{l}\text { MEAN } \\
\text { MINIMUM } \\
\text { MAXIMUM } \\
\text { EXCEPTION } \\
\text { NUMBER }\end{array}$ & $\begin{array}{l}.003 \\
.003 \\
.003 \\
0 \\
1\end{array}$ & $\begin{array}{l}.0006 \\
.0006 \\
.0006 \\
i\end{array}$ & $\begin{array}{l}.002 \\
.002 \\
i .002\end{array}$ & $\begin{array}{l}.005 \\
.005 \\
.005 \\
0 \\
1\end{array}$ & $\begin{array}{c}42.900 \\
42.900 \\
42.900 \\
0 \\
1\end{array}$ & $\begin{array}{l}.005 \\
.005 \\
i .005 \\
i\end{array}$ & $\begin{array}{l}.950 \\
.950 \\
.950 \\
0 \\
1\end{array}$ \\
\hline
\end{tabular}


TABLE 25 (Continued)

1979 BNL ENVIRONMENTAL MONITORING MISCELLANEOUS ON SITE WELLS WATER QUALITY AND PUR! TY-METALS

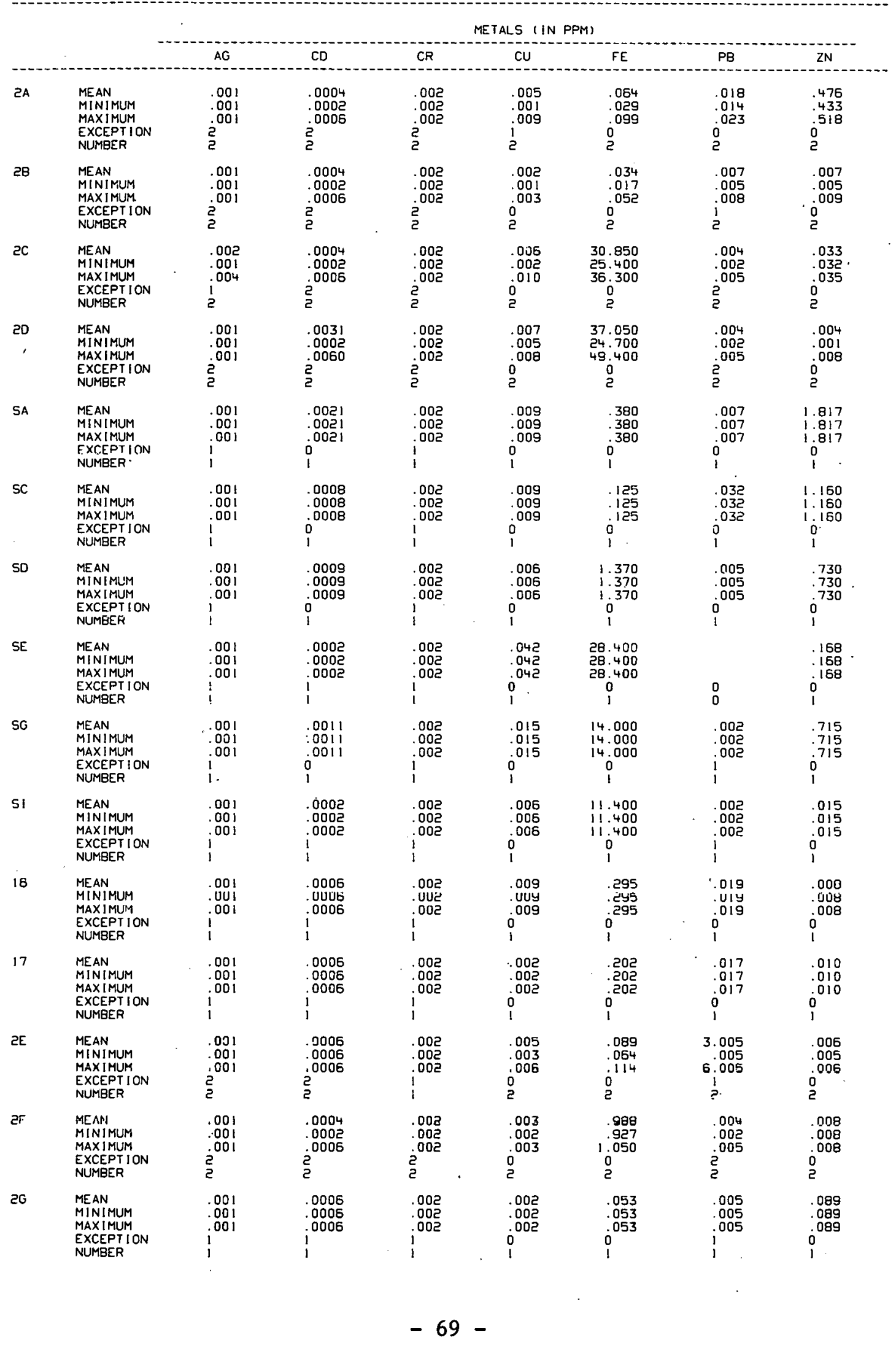


TABLE 25 (Continued)

1979 BNL ENVIRONMENTAL MONITORING WASTE MANAGEMENT AREA WELLS WATER OUALITY AND PUA

\begin{tabular}{|c|c|c|c|c|c|c|c|c|c|}
\hline \multicolumn{10}{|c|}{ METALS (IN PPM) } \\
\hline WB & $\begin{array}{l}\text { MEAN } \\
\text { MINIMUM } \\
\text { MAXIMUM } \\
\text { EXCEPT ION } \\
\text { NUMBER }\end{array}$ & $\begin{array}{l}.001 \\
.001 \\
.001 \\
3 \\
3\end{array}$ & $\begin{array}{l}.0005 \\
.0002 \\
.0006 \\
3 \\
3\end{array}$ & $\begin{array}{l}.002 \\
.002 \\
.002 \\
3 \\
3\end{array}$ & $\begin{array}{l}.005 \\
.002 \\
.010 \\
0 \\
3\end{array}$ & $\begin{array}{l}.123 \\
.039 \\
.258 \\
0 \\
3\end{array}$ & $\begin{array}{l}.006 \\
.005 \\
.005 \\
j \\
3\end{array}$ & $\begin{array}{l}.534 \\
.383 \\
.791 \\
0 \\
3\end{array}$ & \\
\hline WC & $\begin{array}{l}\text { MEAN } \\
\text { MINIMUM } \\
\text { MAXIMUM } \\
\text { EXCEPIIION } \\
\text { NUMBER }\end{array}$ & $\begin{array}{l}.001 \\
.001 \\
i 001 \\
1\end{array}$ & $\begin{array}{l}0^{\circ} \\
0\end{array}$ & $\begin{array}{l}.002 \\
.002 \\
i^{002}\end{array}$ & $\begin{array}{l}.006 \\
.006 \\
.006 \\
0 \\
1\end{array}$ & $\begin{array}{l}.546 \\
.546 \\
.546 \\
0 \\
1\end{array}$ & $\begin{array}{l}.002 \\
.002 \\
.002 \\
0 \\
1\end{array}$ & $\begin{array}{l}.481 \\
.481 \\
.481 \\
0 \\
1\end{array}$ & ' \\
\hline WD & $\begin{array}{l}\text { MEAN } \\
\text { MINIMUM } \\
\text { MAXIMUM } \\
\text { EXCEPT I ON } \\
\text { NUMBER }\end{array}$ & $\begin{array}{l}.001 \\
.001 \\
.001 \\
3 \\
3\end{array}$ & $\begin{array}{l}.0005 \\
.0003 \\
.0005 \\
2 \\
3\end{array}$ & $\begin{array}{l}.002 \\
.001 \\
.002 \\
3 \\
3\end{array}$ & $\begin{array}{l}.008 \\
.007 \\
.009 \\
0 \\
3\end{array}$ & $\begin{array}{l}.156 \\
.068 \\
.260 \\
0 \\
3\end{array}$ & $\begin{array}{l}.013 \\
.011 \\
.016 \\
0 \\
3\end{array}$ & $\begin{array}{l}.897 \\
.794 \\
1.100 \\
0 \\
3\end{array}$ & \\
\hline $\mathrm{HF}^{\mathrm{F}}$ & $\begin{array}{l}\text { MFAN } \\
\text { MINIMIIM } \\
\text { MAXIMUM } \\
\text { EXCEPTION } \\
\text { NUMBER }\end{array}$ & $\begin{array}{l}\text { l111 } \\
\text { nnl } \\
i^{001} \\
i^{0}\end{array}$ & $i^{0002}$ & $\begin{array}{l}.003 \\
.009 \\
i 002 \\
1\end{array}$ & $\begin{array}{l}.003 \\
.003 \\
.003 \\
0 \\
1\end{array}$ & $\begin{array}{l}: 060 \\
.060 \\
.068 \\
0 \\
1\end{array}$ & $\begin{array}{l}.000 \\
.005 \\
i^{002} \\
i\end{array}$ & $\begin{array}{l}.407 \\
.467 \\
.407 \\
0 \\
1\end{array}$ & \\
\hline WJ & $\begin{array}{l}\text { MCAN } \\
\text { liIISiIMIIIM } \\
\text { MAXIMUM } \\
\text { EXCEPTION } \\
\text { NUMBER }\end{array}$ & $\begin{array}{l}.001 \\
.001 \\
.001 \\
1\end{array}$ & $\begin{array}{l}.0002 \\
.0002 \\
.0002 \\
i\end{array}$ & $\begin{array}{l}.002 \\
.012 \\
.002 \\
i^{002}\end{array}$ & $\begin{array}{l}.004 \\
.0011 \\
.004 \\
0 \\
1\end{array}$ & $\begin{array}{l}: 759 \\
: 759 \\
.759 \\
0 \\
1\end{array}$ & $\begin{array}{l}.411 \\
.011 \\
011 \\
0 \\
1\end{array}$ & $\begin{array}{l}.511 \\
.511 \\
.511 \\
0^{1}\end{array}$ & \\
\hline WK & $\begin{array}{l}\text { MEAN } \\
\text { MINIMUM } \\
\text { MAXIMUM } \\
\text { EXCEPTIIUN } \\
\text { NUMBER }\end{array}$ & $\begin{array}{l}.001 \\
.001 \\
.001 \\
3 \\
3\end{array}$ & $\begin{array}{l}.0004 \\
.0002 \\
.0008 \\
3 \\
3\end{array}$ & $\begin{array}{l}.002 \\
.001 \\
.002 \\
3 \\
3\end{array}$ & $\begin{array}{l}.004 \\
.004 \\
.005 \\
0 \\
3\end{array}$ & $\begin{array}{l}.080 \\
.047 \\
.122 \\
0 \\
3\end{array}$ & $\begin{array}{l}.003 \\
.002 \\
.005 \\
2^{3}\end{array}$ & $\begin{array}{l}.656 \\
.430 \\
.86 n \\
0 \\
3\end{array}$ & \\
\hline WL. & $\begin{array}{l}\text { MEAN } \\
\text { MINIMUM } \\
\text { MAXIMUM } \\
\text { EXCEPTION } \\
\text { NUMBER }\end{array}$ & $\begin{array}{l}.001 \\
.001 \\
.001 \\
3 \\
3\end{array}$ & $\begin{array}{l}.0004 \\
.0002 \\
.0006 \\
3 \\
3\end{array}$ & $\begin{array}{l}.002 \\
.001 \\
.002 \\
3 \\
3\end{array}$ & $\begin{array}{l}.021 \\
.003 \\
.056 \\
0 \\
3\end{array}$ & $\begin{array}{l}.173 \\
.068 \\
.342 \\
0 \\
3\end{array}$ & $\begin{array}{l}.003 \\
.002 \\
.005 \\
\dot{2}^{2}\end{array}$ & $\begin{array}{l}.302 \\
.241 \\
.375 \\
0 \\
3\end{array}$ & \\
\hline WM & $\begin{array}{l}\text { MEAN } \\
\text { MINIMUM } \\
\text { MAXIMUM } \\
\text { EXCCRTION } \\
\text { NUMBER }\end{array}$ & $\begin{array}{l}.001 \\
.001 \\
.001 \\
i^{1}\end{array}$ & $\begin{array}{l}.0002 \\
.0002 \\
.0002 \\
i^{1}\end{array}$ & $\begin{array}{l}.002 \\
.002 \\
.002 \\
i \\
1\end{array}$ & $\begin{array}{l}.003 \\
.003 \\
.003 \\
u^{1}\end{array}$ & $\begin{array}{l}.372 \\
.372 \\
372 \\
0^{372} \\
1\end{array}$ & $\begin{array}{l}.002 \\
.002 \\
i^{002} \\
i\end{array}$ & $\begin{array}{l}.159 \\
.159 \\
159 \\
0 \\
1\end{array}$ & \\
\hline WN & 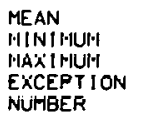 & $\begin{array}{l}.001 \\
.001 \\
i^{001} \\
!\end{array}$ & $\begin{array}{l}.0002 \\
. \text { UUUट } \\
. \text { UUUट } \\
1\end{array}$ & $\begin{array}{l}.002 \\
.002 \\
.002 \\
i^{002}\end{array}$ & $\begin{array}{l}.004 \\
.004 \\
.004 \\
i\end{array}$ & $\begin{array}{l}.569 \\
.569 \\
569 \\
0 \\
0 \\
1\end{array}$ & $\begin{array}{l}.010 \\
.010 \\
.010 \\
0 \\
1\end{array}$ & $\begin{array}{lll}1 & 113 \\
0 & 1 & 13 \\
0 & 113 \\
1\end{array}$ & \\
\hline WU & $\begin{array}{l}\text { MEAN } \\
\text { MINIMUM } \\
\text { MAXIMUM } \\
\text { EXCEPT I ON } \\
\text { NUMBER }\end{array}$ & $\begin{array}{l}.001 \\
.001 \\
i^{001}\end{array}$ & $\begin{array}{l}.0064 \\
.0064 \\
.0064 \\
0 \\
1\end{array}$ & $\begin{array}{l}.002 \\
.002 \\
.002 \\
0 \\
1\end{array}$ & $\begin{array}{l}.005 \\
.005 \\
.005 \\
0 \\
1\end{array}$ & $\begin{array}{l}.471 \\
.171 \\
.471 \\
0 \\
1\end{array}$ & $\begin{array}{l}.002 \\
.002 \\
.002 \\
0 \\
1\end{array}$ & $\begin{array}{c}1 \\
0.210 \\
2.210 \\
2.210 \\
1\end{array}$ & \\
\hline WV & $\begin{array}{l}\text { MEAN } \\
\text { MINIMUM } \\
\text { IIAXIIHUI'I } \\
\text { FXC.FPT INN } \\
\text { NUMBER }\end{array}$ & $\begin{array}{l}.001 \\
.001 \\
\text {. } 001 \\
i^{1}\end{array}$ & $\begin{array}{l}.0006 \\
.0006 \\
.01006 \\
0^{1}\end{array}$ & $\begin{array}{l}.002 \\
.002 \\
.002 \\
i\end{array}$ & $\begin{array}{l}.0 n \pi \\
.005 \\
.006 \\
0 \\
1\end{array}$ & $\begin{array}{l}.205 \\
.205 \\
.205 \\
0 \\
1\end{array}$ & $\begin{array}{l}.005 \\
.005 \\
.005 \\
0 \\
1\end{array}$ & $\begin{array}{l}2.100 \\
3.100 \\
2.100 \\
0 \\
1\end{array}$ & \\
\hline WW & $\begin{array}{l}\text { MEAN } \\
\text { MINIMUM } \\
\text { MAXIMUM } \\
\text { EXCEPTION } \\
\text { NUMBER }\end{array}$ & $\begin{array}{l}.001 \\
.001 \\
i^{001} \\
1\end{array}$ & $\begin{array}{l}.0004 \\
.0004 \\
.0004 \\
0 \\
1\end{array}$ & $\begin{array}{l}.002 \\
.002 \\
002 \\
i^{002}\end{array}$ & $\begin{array}{l}.004 \\
.004 \\
.004 \\
0 \\
1\end{array}$ & $\begin{array}{l}.049 \\
.049 \\
.049 \\
0 \\
1\end{array}$ & $\begin{array}{l}.010 \text { म } \\
.000 \\
.008 \\
0 \\
1\end{array}$ & $\begin{array}{l}105 n \\
1.050 \\
1.050 \\
0 \\
1\end{array}$ & \\
\hline WX & $\begin{array}{l}\text { MEAN } \\
\text { MINIMUMY } \\
\text { MAXIMUM } \\
\text { EXCEPT ION } \\
\text { NUMBER }\end{array}$ & $\begin{array}{l}.001 \\
.001 \\
i^{001}\end{array}$ & $\begin{array}{l}.0002 \\
.0009 \\
i^{.0002}\end{array}$ & $\begin{array}{l}.002 \\
.000 \\
.002 \\
i\end{array}$ & $\begin{array}{l}.004 \\
0004 \\
.004 \\
0 \\
1\end{array}$ & $\begin{array}{l}.159 \\
.150 \\
.159 \\
0^{159}\end{array}$ & $\begin{array}{l}.007 \\
.007 \\
007 \\
0 \\
1\end{array}$ & $\begin{array}{l}1.730 \\
1.730 \\
1.730 \\
0 \\
1\end{array}$ & \\
\hline WZ & $\begin{array}{l}\text { MEAN } \\
\text { MINIMUM } \\
\text { MAXIMUM } \\
\text { EXCEPT ION } \\
\text { NUMBER }\end{array}$ & $\begin{array}{l}.001 \\
.001 \\
i^{001} \\
1\end{array}$ & $\begin{array}{l}.0039 \\
.0039 \\
.0039 \\
0 \\
1\end{array}$ & $\begin{array}{l}.002 \\
.002 \\
.002 \\
1 \\
1\end{array}$ & $\begin{array}{l}0 \\
0\end{array}$ & $\begin{array}{c}4.450 \\
4.450 \\
4.450 \\
0 \\
1\end{array}$ & $\begin{array}{l}.142 \\
: 142 \\
0142 \\
0 \\
1\end{array}$ & $\begin{array}{c}2.820 \\
2.820 \\
2.820 \\
0 \\
1\end{array}$ & \\
\hline W! & $\begin{array}{l}\text { MEAN } \\
\text { MINIMUM } \\
\text { MAXIMUM } \\
\text { EXCEPT ION } \\
\text { NUMBER }\end{array}$ & $\begin{array}{l}.001 \\
.001 \\
.001 \\
3 \\
3\end{array}$ & $\begin{array}{l}.0004 \\
.0002 \\
.0006 \\
3^{3}\end{array}$ & $\begin{array}{l}.002 \\
.001 \\
.002 \\
2^{3}\end{array}$ & $\begin{array}{l}.012 \\
.003 \\
.038 \\
0 \\
4\end{array}$ & $\begin{array}{l}.134 \\
.078 \\
.190 \\
0 \\
2\end{array}$ & $\begin{array}{l}.003 \\
.002 \\
.005 \\
i^{1}\end{array}$ & $\begin{array}{l}.440 \\
.360 \\
.520 \\
0^{\circ}\end{array}$ & \\
\hline
\end{tabular}


TABLE 25 (Continued)

1979 BNL ENVIRONMENTAL MONITORING FORMER DUMP AREA WELLS WATER QUALITY AND PURITY-METALS

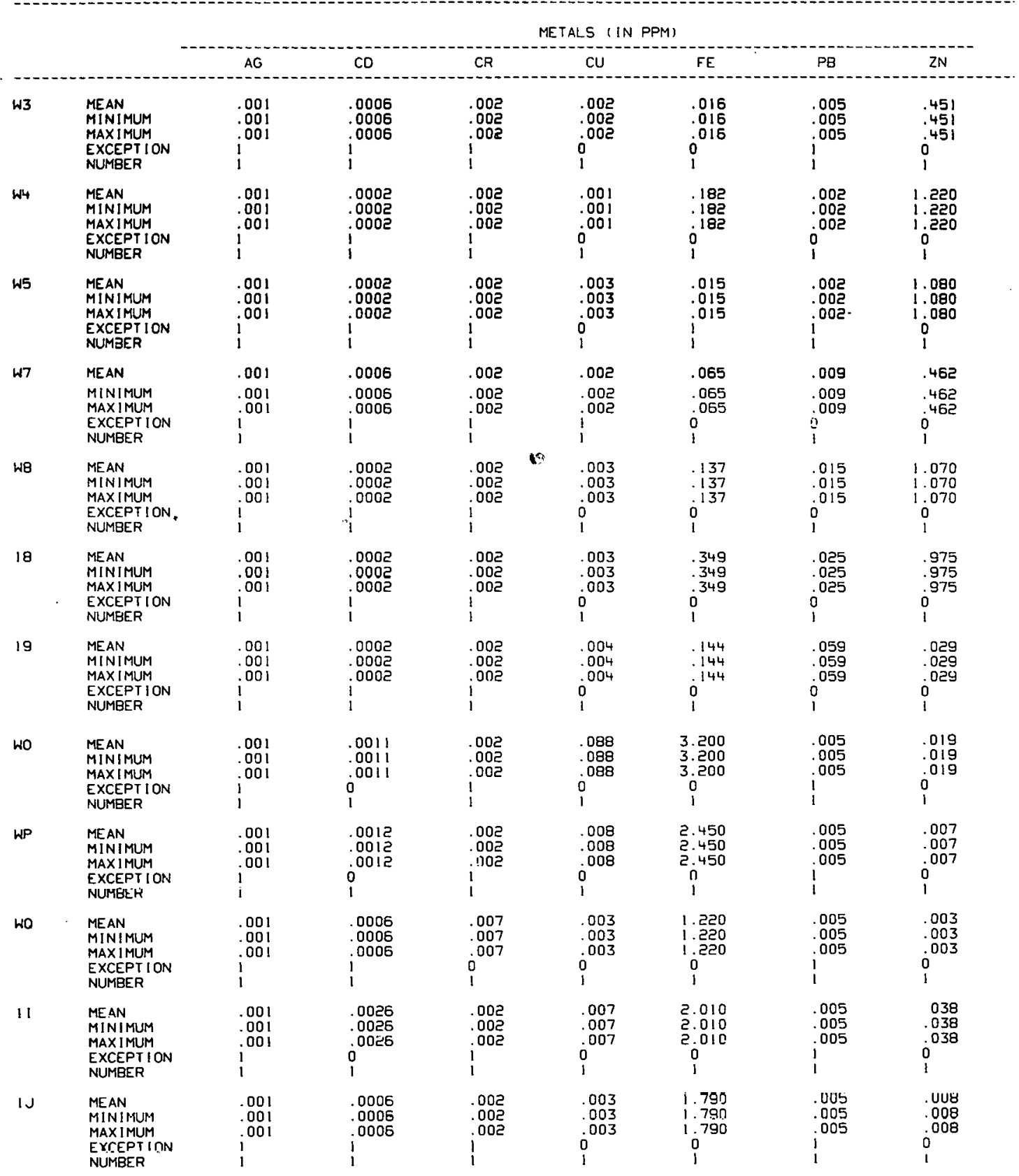


TABLE 25 (Continued)

1979 BNL ENVIRONMENTAL MONITORING BUILOING 650 SUMP AREA WELLS WATER QUA'.ITY AND PURITY-METALS

\begin{tabular}{|c|c|c|c|c|c|c|c|c|}
\hline & & \multicolumn{7}{|c|}{ METALS (IN PPM) } \\
\hline & & $A C$ & $\mathrm{CD}$ & $\mathrm{CR}$ & $\mathrm{CU}$ & FE & $\mathrm{PB}$ & Ziv \\
\hline IA & $\begin{array}{l}\text { MEAN } \\
\text { MINIMUN } \\
\text { IFAXIMUI } \\
\text { EXCEPT ION } \\
\text { NUMBER }\end{array}$ & $\begin{array}{l}.001 \\
.001 \\
.0011 \\
i\end{array}$ & $\begin{array}{l}.0002 \\
.0002 \\
.0002 \\
i\end{array}$ & $\begin{array}{l}.002 \\
.002 \\
i \\
i \\
i 00\end{array}$ & $\begin{array}{l}.003 \\
.003 \\
.003 \\
u \\
\text { u }\end{array}$ & $\begin{array}{l}.250 \\
.250 \\
.250 \\
u^{1} \\
1\end{array}$ & $\begin{array}{l}.006 \\
.006 \\
.006 \\
0 \\
1\end{array}$ & $\begin{array}{l}.845 \\
.845 \\
.845 \\
0 \\
1\end{array}$ \\
\hline IC & $\begin{array}{l}\text { MEAN } \\
\text { MINIMUM } \\
\text { MAXIMUM } \\
\text { EXSEOT I DN } \\
\text { NUMBER }\end{array}$ & $\begin{array}{l}.001 \\
.001 \\
i^{001} \\
i\end{array}$ & $\begin{array}{l}.0002 \\
.0002 \\
i \\
i\end{array}$ & $\begin{array}{l}.002 \\
.002 \\
i 002 \\
10\end{array}$ & $\begin{array}{l}.004 \\
.004 \\
.004 \\
0 \\
1\end{array}$ & $\begin{array}{l}.481 \\
.481 \\
.481 \\
0 \\
1\end{array}$ & $\begin{array}{l}.033 \\
.033 \\
.033 \\
0 \\
1\end{array}$ & $\begin{array}{l}.953 \\
.953 \\
.953 \\
0 \\
1\end{array}$ \\
\hline $1 \mathrm{D}$ & $\begin{array}{l}\text { MEAN } \\
\text { MINIMUM } \\
\text { MAXIMUM } \\
\text { EXCEPT ION } \\
\text { NUMBER }\end{array}$ & $\begin{array}{l}.001 \\
.001 \\
i .001 \\
i\end{array}$ & $\begin{array}{l}.0002 \\
.0002 \\
i^{0002} \\
1\end{array}$ & $\begin{array}{l}.002 \\
.002 \\
i 002 \\
i \\
1\end{array}$ & $\begin{array}{l}.004 \\
.004 \\
.004 \\
0 \\
1\end{array}$ & $\begin{array}{l}.068 \\
.068 \\
.068 \\
0 \\
1\end{array}$ & $\begin{array}{l}.009 \\
.009 \\
.009 \\
0 \\
1\end{array}$ & $\begin{array}{l}.858 \\
.858 \\
.858 \\
0 \\
1\end{array}$ \\
\hline IE & $\begin{array}{l}\text { MEAN } \\
\text { MIN[MUM } \\
\text { MAXIMUM } \\
\text { EXCE'TION } \\
\text { NUMBER }\end{array}$ & $\begin{array}{l}.001 \\
.001 \\
.001 \\
i\end{array}$ & $\begin{array}{l}.0008 \\
.0008 \\
.0008 \\
0^{1}\end{array}$ & $\begin{array}{l}.002 \\
.002 \\
i^{.002} \\
i\end{array}$ & $\begin{array}{l}.011 \\
.011 \\
011 \\
0 \\
1\end{array}$ & $\begin{array}{l}.263 \\
.263 \\
.263 \\
0 \\
1\end{array}$ & $\begin{array}{l}.035 \\
.035 \\
.035 \\
0 \\
1\end{array}$ & $\begin{array}{l}2.900 \\
2.900 \\
2.900 \\
0 \\
1\end{array}$ \\
\hline IH & $\begin{array}{l}\text { MEAN } \\
\text { MINIMUM } \\
\text { MAXIMUM } \\
\text { EXCEPT ION } \\
\text { NUMBEH }\end{array}$ & $\begin{array}{l}.001 \\
.001 \\
.001 \\
i\end{array}$ & $\begin{array}{l}.0002 \\
.0002 \\
i^{0002}\end{array}$ & $\begin{array}{l}.002 \\
.002 \\
.002 \\
i\end{array}$ & $\begin{array}{l}.076 \\
.076 \\
.076 \\
0 \\
1\end{array}$ & $\begin{array}{l}.263 \\
.263 \\
.263 \\
0 \\
i\end{array}$ & $\begin{array}{l}.036 \\
.036 \\
.036 \\
0 \\
1\end{array}$ & $\begin{array}{l}3.160 \\
3.150 \\
3.150 \\
0 \\
1\end{array}$ \\
\hline
\end{tabular}

Reference Standards - Table 29

Number: samples analyzed per year

Exception: sample concentration $<M D L$ 
TABLE 26

1979 BNL ENVIRONMENTAL MONITORING POTABLE SUPPLY WELLS WATER OUALITY AND PURITY-METALS

\begin{tabular}{|c|c|c|c|c|c|c|c|c|}
\hline & & $A G$ & $\mathrm{CD}$ & \multicolumn{3}{|c|}{ METALS (IN PPM) } & $\mathrm{PB}$ & $Z N$ \\
\hline $\mathrm{FB}$ & $\begin{array}{l}\text { MEAN } \\
\text { MINIMUM } \\
\text { MAXIMUM } \\
\text { EXCEPT ION } \\
\text { NUMBER }\end{array}$ & $\begin{array}{l}.001 \\
.001 \\
.001 \\
4 \\
4\end{array}$ & $\begin{array}{l}.0004 \\
.0002 \\
.0006 \\
4 \\
4\end{array}$ & $\begin{array}{l}.002 \\
.002 \\
.002 \\
3 \\
4\end{array}$ & $\begin{array}{l}.018 \\
.015 \\
.023 \\
0 \\
4\end{array}$ & $\begin{array}{l}2.463 \\
1.340 \\
5.630 \\
0 \\
4\end{array}$ & $\begin{array}{l}.004 \\
.002 \\
.005 \\
4 \\
4\end{array}$ & $\begin{array}{l}.007 \\
.005 \\
.012 \\
0 \\
4\end{array}$ \\
\hline FO & $\begin{array}{l}\text { MEAN } \\
\text { MINIMUM } \\
\text { MAXIMUM } \\
\text { EXCEPTION } \\
\text { NUMBER }\end{array}$ & $\begin{array}{l}.001 \\
.001 \\
.001 \\
4 \\
4\end{array}$ & $\begin{array}{l}.0011 \\
.0002 \\
.0030 \\
4 \\
4\end{array}$ & $\begin{array}{l}.002 \\
.002 \\
.003 \\
3 \\
4\end{array}$ & $\begin{array}{l}.015 \\
.003 \\
.036 \\
0 \\
4\end{array}$ & $\begin{array}{c}2.913 \\
.533 \\
5.000 \\
0 \\
4\end{array}$ & $\begin{array}{l}.004 \\
.002 \\
.005 \\
4 \\
4\end{array}$ & $\begin{array}{l}.004 \\
.001 \\
.007 \\
i^{4}\end{array}$ \\
\hline FE & $\begin{array}{l}\text { MEAN } \\
\text { MINIMUM } \\
\text { MAXIMUM } \\
\text { EXCEPT ION } \\
\text { NUMBER }\end{array}$ & $\begin{array}{l}.001 \\
.001 \\
.001 \\
4 \\
.4\end{array}$ & $\begin{array}{l}.0004 \\
.0002 \\
.0006 \\
4 \\
4\end{array}$ & $\begin{array}{l}.002 \\
.001 \\
.002 \\
4 \\
4\end{array}$ & $\begin{array}{l}.006 \\
.002 \\
.011 \\
0 \\
4\end{array}$ & $\begin{array}{l}.419 \\
.129 \\
.812 \\
0 \\
4\end{array}$ & $\begin{array}{l}.003 \\
.002 \\
.005 \\
4 \\
4\end{array}$ & $\begin{array}{l}.100 \\
.069 \\
.133 \\
0 \\
4\end{array}$ \\
\hline FF & $\begin{array}{l}\text { MEAN } \\
\text { MINIMUM } \\
\text { MAXIMUM } \\
\text { EXCEPT ION } \\
\text { NUMBER }\end{array}$ & $\begin{array}{l}.001 \\
.001 \\
.001 \\
3 \\
4\end{array}$ & $\begin{array}{l}.0011 \\
.0002 \\
.0030 \\
3 \\
4\end{array}$ & $\begin{array}{l}.002 \\
.002 \\
.002 \\
3 \\
4\end{array}$ & $\begin{array}{l}.006 \\
.002 \\
.015 \\
0 \\
4\end{array}$ & $\begin{array}{c}5.140 \\
3.830 \\
5.910 \\
0 \\
4\end{array}$ & $\begin{array}{l}.005 \\
.002 \\
.010 \\
3 \\
4\end{array}$ & $\begin{array}{l}.002 \\
.001 \\
.005 \\
2 \\
4\end{array}$ \\
\hline FG & $\begin{array}{l}\text { MEAN } \\
\text { MINIMUM } \\
\text { MAXIMUM } \\
\text { EXCEPT ION } \\
\text { NUMBER }\end{array}$ & $\begin{array}{l}.001 \\
.001 \\
.001 \\
3 \\
3\end{array}$ & $\begin{array}{l}.0004 \\
.0002 \\
.0006 \\
3 \\
3\end{array}$ & $\begin{array}{l}.008 \\
.002 \\
.021 \\
2 \\
3\end{array}$ & $\begin{array}{l}.004 \\
.003 \\
.005 \\
0 \\
3\end{array}$ & $\begin{array}{l}1.823 \\
1.730 \\
2.000 \\
0 \\
3\end{array}$ & $\begin{array}{l}.003 \\
.002 \\
.005 \\
3 \\
3\end{array}$ & $\begin{array}{l}.008 \\
.007 \\
.010 \\
0 \\
3\end{array}$ \\
\hline
\end{tabular}

1979 BNL ENVIRONMENTAL MONITORING COOLING WATER WELLS WATER QUALITY AND PURITY-METALS

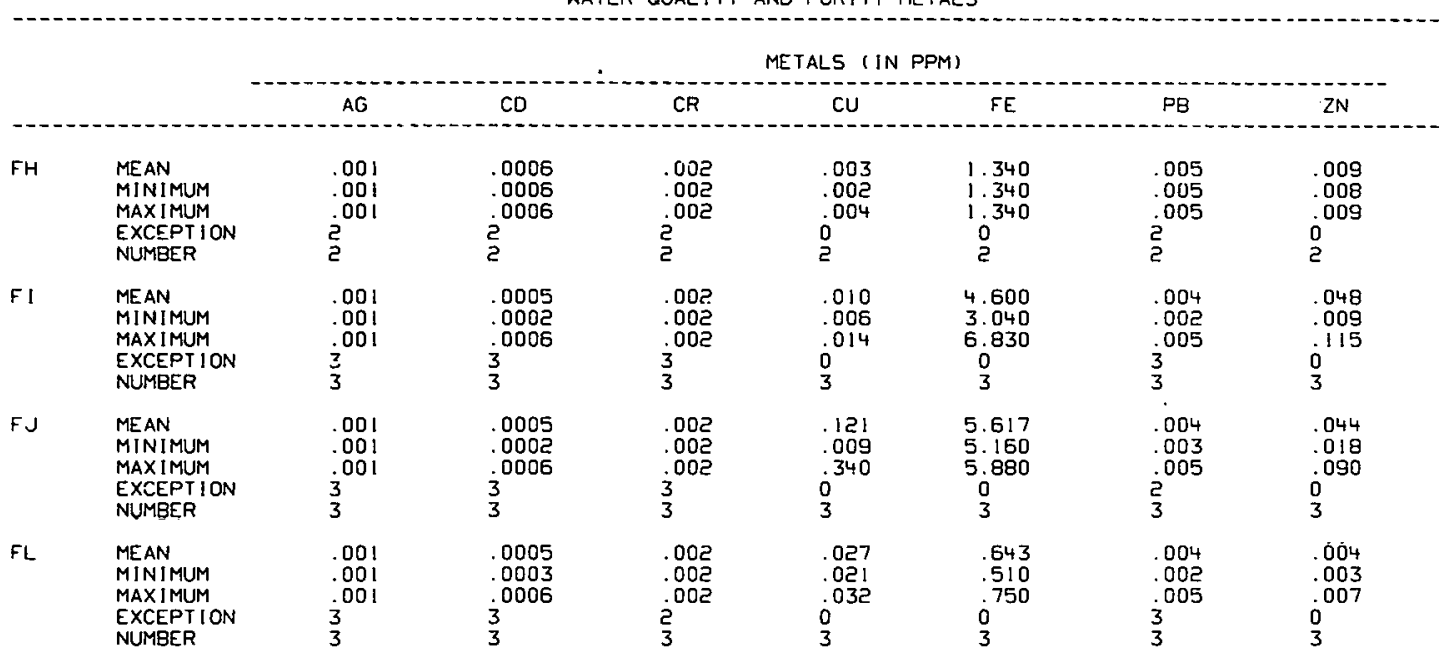

Reference Standards - Table 29

Locations indicated on Fig. 8 \& ldentifled as:

Number: samples analyzed per year

Potable Cooling

Exception: sample concentration

$\begin{array}{ll}\text { FA: } 1 & \text { FH: } 101 \\ \text { FB: } & \text { FI: } 102 \\ \text { FC: } 3 & \text { FJ: } 103 \\ \text { FD: } 4 & \text { FK: } 104 \\ \text { FE } 5 & \text { FL: } 105 \\ \text { FF }: 6 & \\ \text { FG }: 7 & \end{array}$


elements in the ground water system by means of the Laboratory surveillance we 11 s downstream in the direction of the ground water flow, has indicated significant decreases as we proceed away from the Laboratory, such as $60 \%$ along the Peconic River, 25 to $30 \%$ in the waste management area and $50 \%$ in the 650 sump area. Investigations into the Laboratory wide use of $z$ inc-containing chemicals focused our attention on a compound used as a cleaning agent for cooling towers in the past. A recent Laboratory analysis of this compound indicated a concentration of zinc to be about $3 \mathrm{mg} / \mathrm{ml}$. It was gathered from discussions with Plant Engineering that the washings were discharged into the sewage system. seems possible that this input may be retained in the sand filter beds and leached into the ground water system, thereby contributing to the increases noted. The Industrial Hygiene Group of the Safety and Environmental Protection Division has instituted a program whereby purchases of chemical compounds that have the potential of polluting the river water are flagged and the user is notified of the proper disposal method. This program has helped the Safety and Environmental Protection Division to identify and advise the users on a score of such compounds since 1978. In ddiliun, a Laburalury wide nutificacion program has been put into effect whereby permission is required from Safety and Environmental Protection Division before any chemical that is defined as toxic is discharged into the sanitary system.

Much lower levels of $\mathrm{Zn}$ were found in the Laboratory supply wells. Several contain $\mathrm{Fe}$ in excess of the standard, but most of this is removed prior to use. $\mathrm{Zn}$ and $\mathrm{Fe}$ are considered as nuisance elements and not as a health hazard.

A depiction of the general direction and rate of ground water movement, originally published in the U.S. Geological Survey Study, is shown in Figure 11 . The Upland Recharge Project (26) has determined a ground water velocity of 13.4 $\mathrm{cm} \mathrm{d}^{-1}$ which is in good agreement with the U.S. Geological Survey Study estimate of $16.2 \mathrm{~cm} \mathrm{~d}^{-1}(7)$. Thus, it appears that many years of travel time would be required for ground water containing radioactivity or other pollutants to reach an off-site we1l, during which considerable dilution by infiltration of precipitation would be anticipated. The data from all the surveillance wells are reviewed at trequent intervals in order to evaluate the monitoring program and appropriate action is taken, such as, rescheduling the sampling of wells and follow up analysis if required.

\section{$3.4 \quad$ Unusual Occurrences:}

\subsubsection{Oil Spills}

During 1979, the Laboratory experienced six oil spills. They occurred at research facilities and at the steam plant. At the research facilities, the spills were restricted to scintillating fluids, whereas at the steam plant it was mainly 非 oil mixed with ALF. Reporting and clean-up procedures were instituted immediately. The absorbents used to clean up the spills were disposed of according to New York State Department of Environmental Conservation (NYSDEC) approved procedures. Appropriate action pertaining to revegetation is in progress. Followup on the two oil spills that occurred in 1977 has indicated that the actions taken by $P$ lant Engineering ( $P E)$ in fertilizing the region and tilling the soil has aided in the biodegradation of the oil. In addition, grass 


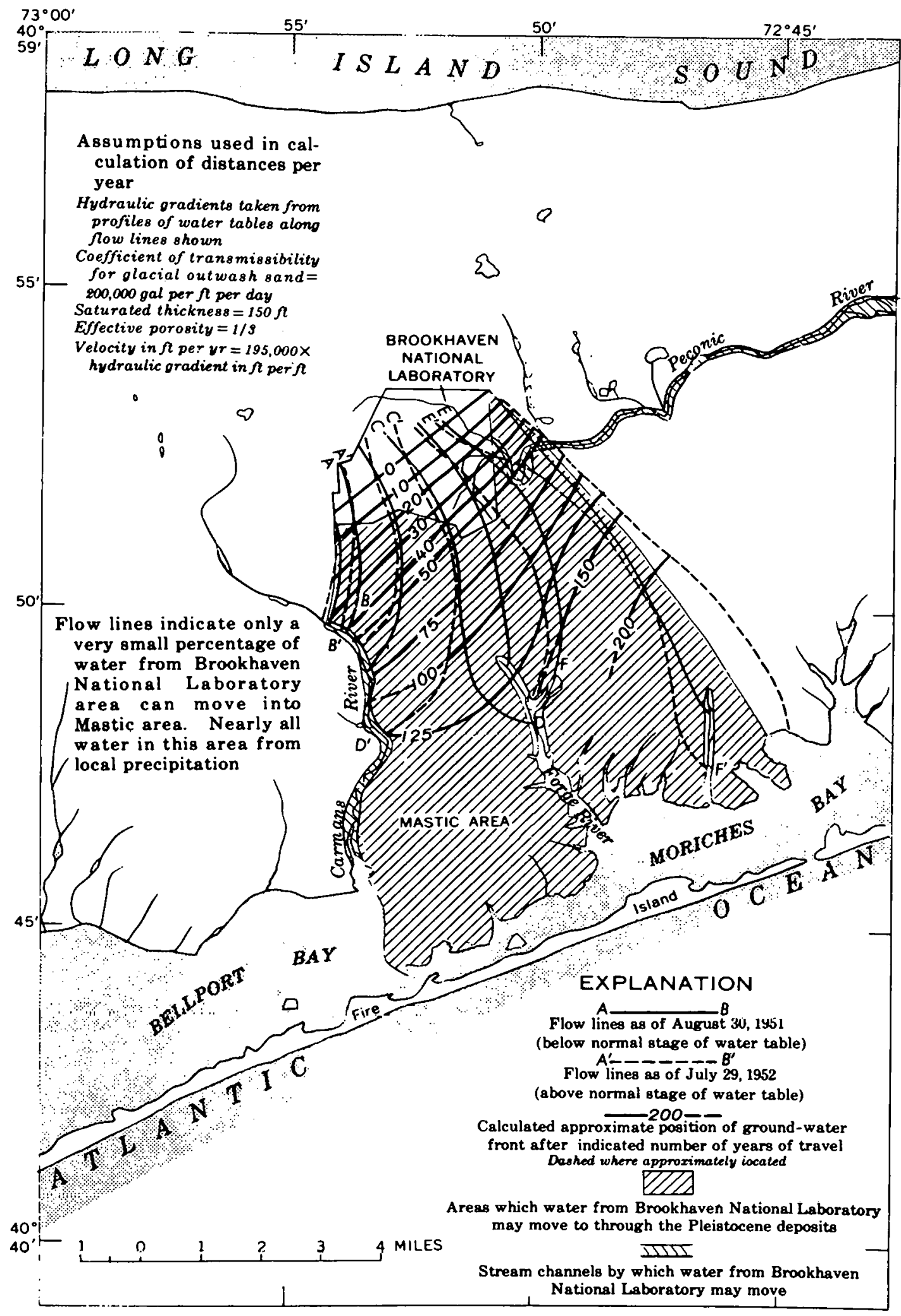

Figure 11. Direction and Time of Travel of Ground Water Laterally in Upper Pleistocene Deposits from the Brookhaven National Laboratory Area to Points of Discharge 
seeding has almost returned the surface to normal conditions. Those wells adjoining the steam plant near the recent spills have given no indication that any oil or its compounds have broken through the retaining clay barrier.

Monitoring will be continued on a regular basis in order to detect movement of oil, if any, in the ground water system.

\subsubsection{Chinese Nuclear Tests}

No atmospheric nuclear tests were detonated by the Chinese during 1979 . As indicated in Section 3, slight increases in gross beta activity were noted in air samples and precipitation and a very slight increase over the MDL was detected for $140 \mathrm{Ba}-\mathrm{La}$ in precipitation. Fallout radionuclide concentrations were at or below MDL in milk and grass samples collected from dairy farms in the vicinity of the site. Unlike the previous years, 1976 in particular, the 1979 values may be considered as insignificant in terms of a health hazard.

\subsection{OFF SITE DOSE ESTIMATES}

Increased levels of radiation and concentrations of radioactivity, in air and water, above ambient background, with resulting increased doses to people, are attributable to the following four Laboratory sources:

1. airborne radioactive effluents, primarily tritium,

2. radioactive liquid effluents,

3. the ${ }^{137} \mathrm{Cs}$ source in the Biology Department Ecology Forest,

4. skyshine from the Alternating Gradient Synchrotron (AGS).

These are discussed below, and the collective dose equivalent rate due to Laboratory operations during 1979 is calculated.

\subsection{Annual Average Collective Dose Equivalent Rate Due to Airborne Effluents}

As indicated in Table 4, a total of $225 \mathrm{Ci}\left(8.4 \times 10^{12} \mathrm{~Bq}\right)$ of tritium was released from various Laboratory facilities during 1979 , making it the 1 argest source of dose equivalent relative to other laboratory released radionuclides, to persons off-site. In using this figure to estimate dose equivalent, it was conservatively as sumed that all the tritium released was in the form of tritiated water vapor at the site boundary.

Air activity concentrations of tritium vapor at the site boundary were so low that measurement was difficult. Data given in Table 8 indicate an average concentration (including background) of $6.5 \mathrm{pCi} \mathrm{m}^{-3}\left(0.24 \mathrm{~Bq} \mathrm{~m}^{-3}\right.$ ) at the site boundary ( 2500 meters from the HFBR stack). Continuous exposure at the Radiation Concentration Guide $\left(2 \times 10^{5} \mathrm{pCi} \mathrm{m}^{-3}\right.$ or $\left.7.4 \times 10^{3} \mathrm{~Bq} \mathrm{~m}{ }^{-3}\right)$ would result in a per caput annual average dose equivalent rate of $500 \mathrm{mRem} \mathrm{a} \mathrm{a}^{-1}\left(5 \times 10^{-3} \mathrm{~Sv}\right.$ person $\left.{ }^{-1} a^{-1}\right)$. Thus, the per caput annual average dose equivalent rate at this distance attributable to Laboratory air effluent tritium vapor was $(6.5 \mathrm{x}$ $500) /\left(2 \times 10^{5}\right)$ or $0.02 \mathrm{mRem} \mathrm{a}^{-1}\left(0.2 \times 10^{-6} \mathrm{~Sv}\right.$ person-1 $\left.\mathrm{a}^{-1}\right)$ or 0.005 of the Ra- 
diation Protection Standard (17). Since the individual external background per -aput dose equivalent rate (Table 2) in this area was about 57.7 mRem $a^{-1}(5.77$

$10^{-4} \mathrm{~Sv}$ person ${ }^{-1} \mathrm{a}^{-1}$ ), the tritium contribution amounts to an increase at the site boundary of about $0.1 \%$, which is within the temporal and spatial variations of the background itself.

As was previously stated, the dose equivalents due to ${ }^{41} \mathrm{Ar},{ }^{15} \mathrm{O}$ and ${ }^{127} \mathrm{Xe}$ were considered insignificant and as such were not included in the final estimates.

Routine analyses for air particulate radioactivity and for radiohalogens were made throughout 1979 on air samples collected at several locations. Table 27 gives the doses to the general public due to BNL tritium releases. It indicates that beyond the site boundary, the dose rates due to tritium in air effluents from the Laboratory were very small, compared with background and variations in background. The parameter $X / Q$, tabulated in the second column, is the ratio of ground level concentration to rate of emission, i.e., concentration per unit emission rate, and is a function of meteorological conditions and distance from the source. The values have been calculated for the $97.5 \mathrm{~m}$ release height of the HFBR stack and are averages for a whole year and for all the sixteen tabulated directions. While their use produces an underestimate at close-in distances for releases from shorter stacks, overall it results in some overestimation of population exposure, since $X / Q$ values in the direction of major population centers to the west of the Laboratory are lower than the $360^{\circ}$ averages. Values of the dose rate due to tritium are derived by multiplying the measured values for the 1.6 to $3.2 \mathrm{~km}$ interval $\left(0.019 \mathrm{mRem} \mathrm{a}^{-1}\right)$ by the appropriate ratios of $X / Q$. The collective average dose equivalent rate (total population dose rate) due to the Laboratory tritium effluent was $4.95 \mathrm{rem} \mathrm{a}^{-1}$, and that due to natural background $\left(57.7 \mathrm{mRem} \mathrm{a}^{-1}\right)$ is estimated to be $278,405 \mathrm{rem}$ $a^{-1}$.

\subsection{Doses Due to Liquid Effluents}

Since the Peconic River is not utilized as a drinking water supply, nor for irrigation, its waters do not constitute a direct pathway for the ingestion of radioactivity. However, the upper portions of the river are utilized for occasional recreational fishing.

Based on observations, discussions with the New York State Department of Environmental Conservation, and the productivity of the Peconic River, an annual total catch of $500 \mathrm{~kg}$ of $\mathrm{fish}$ is reasonable. If one assumes that $100 \mathrm{fishermen}$ (who are being treated as maximum individuals) catch the above amount of fish and that their families consume all the fish caught and, furthermore, the breakdown of adults and children (based on an average family of 2 adults and 2 children) to be 354 adults and children above 12 years and 56 infants $(3,4)$, then the annual consumption of fish by the adult group is $1.36 \mathrm{~kg} / \mathrm{yr}$ and infants is $0.46 \mathrm{~kg} / \mathrm{yr}$ (as opposed to the USNRC Regulatory Guide (24) value of $21 \mathrm{~kg} / \mathrm{yr}$ and $6.9 \mathrm{~kg} / \mathrm{yr}$ ), respectively. Using the above more realistic value for consumption of fish, the other assumptions recommended in the NRC Regulatory Guide 1.109 (24) and the maximum observed concentration of ${ }^{90} \mathrm{Sr}$ and ${ }^{137} \mathrm{Cs}$ in fish 
Table 27

1979 BNL Environmental Monitoring Collective Annual Average Dose Equivalent Rate Due to BNL Airborne Effluents in Comparison with Background

\begin{tabular}{|c|c|c|c|c|c|}
\hline $\begin{array}{l}\text { Distance } \\
\text { from HFBR } \\
\text { Stack } \\
\text { (km) }\end{array}$ & $X / Q[27]$ & Populationa & $\begin{array}{l}\text { HTO } \\
\text { Per Caput } \\
\text { Dose } \\
\text { Equivalent } \\
\text { Rat.e } \\
\text { Iuvinu } \\
\text { Person-1 }{ }^{-1}=1\end{array}$ & $\begin{array}{l}\text { HTO } \\
\text { Collective } \\
\text { Average } \\
\text { Dose } \\
\text { Finlivilinl } \\
\text { Kare } \\
\text { rem } a^{-1}\end{array}$ & $\begin{array}{l}\text { Background } \\
\text { Collective } \\
\text { Average } \\
\text { Dose } \\
\text { Filuivilent } \\
\text { kaze } \\
\text { rem a-1 }\end{array}$ \\
\hline $1.6-3.2$ & $2.4 \times 10^{-1}$ & 1,597 & 0.019 & 0.03 & $\cdot$ \\
\hline $3.2-4.8$ & $1.0 \times 10^{-7}$ & 5,540 & 0.008 & 0.04 & 321 \\
\hline $4.8-6.4$ & $6.0 \times 10^{-8}$ & 11,592 & 0.005 & 0.06 & 672 \\
\hline $6.4-8.0$ & $3.9 \times 10^{-8}$ & 20,218 & 0.003 & 0.06 & 1,172 \\
\hline $8.0-16.1$ & $1.7 \times 10^{-8}$ & 228,445 & 0.001 & 0.23 & 13,249 \\
\hline $16.1-24.2$ & $8.0 \times 10^{-9}$ & 243,809 & 0.001 & 0.24 & 14,140 \\
\hline $24.2-32.2$ & $5.5 \times 10^{-9}$ & 155,230 & 0.001 & 0.16 & 9.003 \\
\hline $32.2-48.4$ & $3.8 \times 10^{-9}$ & 999,352 & 0.001 & 1.00 & 57,962 \\
\hline $40.4 \cdot 64.5$ & $2.7 \times 10^{-9}$ & $1,302,657$ & 0.001 & 1.38 & 80,194 \\
\hline $64.5-80.6$ & $2.1 \times 10^{-9}$ & $1,751,706$ & 0.001 & 1.75 & 101,599 \\
\hline $1.6-80.6$ & - & $4,800,146$ & - & 4.95 & 278,405 \\
\hline
\end{tabular}

apopulation data estimated from information suppliedby Ms. Peggy Wagner, Research Analyst, Long Island Regional planning Board [3,4]. Scc Tablc 1 for cstimatcd population distribution for 1979.

rem $=0.01 \mathrm{sv}$ 
(Table 18), the estimated maximum individual dose equivalent rate is tabulated el ow.

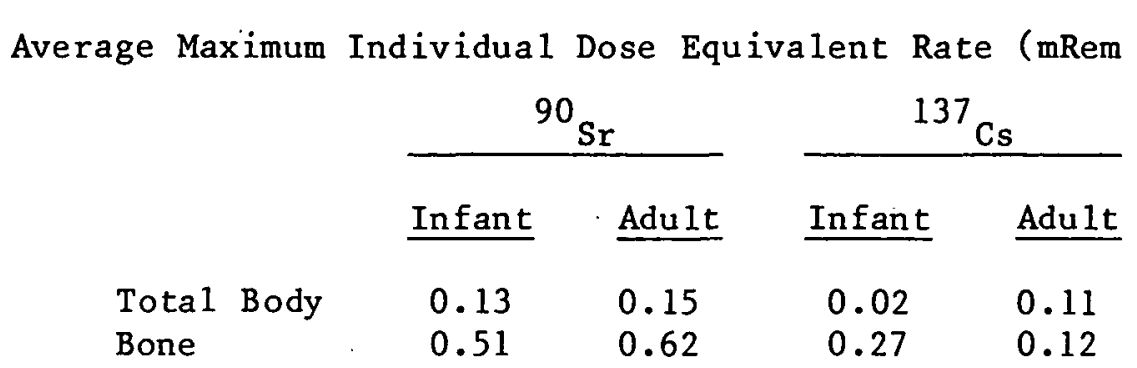

The collective average dose equivalent rate (total dose) from this indirect pathway, for the above population, can be estimated to be $0.35 \mathrm{rem} \mathrm{a}^{-1}$ ( $1.00 \mathrm{mRem} \times 354$ persons) for adults and 0.05 rem $\mathrm{a}^{-1}$ ( $0.93 \mathrm{mRem} \times 56$ persons) for infants.

A1though not directly relatable to the Laboratory liquid effluents during 1979 , a ${ }^{90} \mathrm{Sr}$ concentration of $2 \mathrm{pCi} 1^{-1}\left(0.73 \times 10^{-1} \mathrm{~Bq} 1^{-1}\right)$ was found in an off-site surveillance well (XS) about $0.35 \mathrm{~km}$ east of the Laboratory site boundary along the Peconic River. This level corresponds to $25 \%$ of the EPA Drinking Water Standard (18). If we assume that during 1979 all the 25 people $(3,4) 1$ iving in the vicinity of this we 11 obtained their drinking water from shallow water supply wells containing ${ }^{90} \mathrm{Sr}$ in a concentration equal to that of the surveillance well then the collective average dose equivalent rate (total dose commitment) will not exceed $0.02 \mathrm{rem} \mathrm{a}$ ( 1 ince $8 \mathrm{pCi} / 1$ corresponds to $4 \mathrm{mRem}$ ). Their collective average dose equivalent rate (total dose) from natural background (including internal radiation) would have been about 2.25 rem $a^{-1}$ (person-rem) during 1979.

4.3 Doses Due to the Gamma Forest ${ }^{137}$ Cs Source

A $5883 \mathrm{Ci} * 137 \mathrm{Cs}$ source is located in the northeast part of the Laboratory site, 1010 meters from the north boundary. The dose rate at this boundary during 1979, as determined by the Laboratory Environmental Monitoring Group, was $1.43 \mathrm{mRem} \mathrm{a}^{-1}\left(1.43 \times 10^{-5} \mathrm{~Sv}\right)$, or $0.4 \%$ of the Radiation Protection Standard.

Population doses beyond the site boundary due to this source have been computed using an estimated population count by segments centered on the HFBR stack. Average dose rates for each population segment and for each distance from the source are given in Table 28.

Since the dose rate from this source decreases very rapidly with distance, only population segments located within $5 \mathrm{~km}$ from the source were considered. The off-site collective average dose equivalent (total dose) is 0.05 rem $a^{-1}$ (person-rem $a^{-1}$ ), and appreciable contributions are found only in the $\mathrm{NNE}$ and NE sectors.

$\overline{* \text { As of } 1 / 1 / 79}$ 
TABLE 28

1979 BNL Environmental Monitoring Off-Site Collective Annual Average Dose Equivalent Rate Due to External Radiation Exposure Resulting from the Gamma Forest and AGS Operations

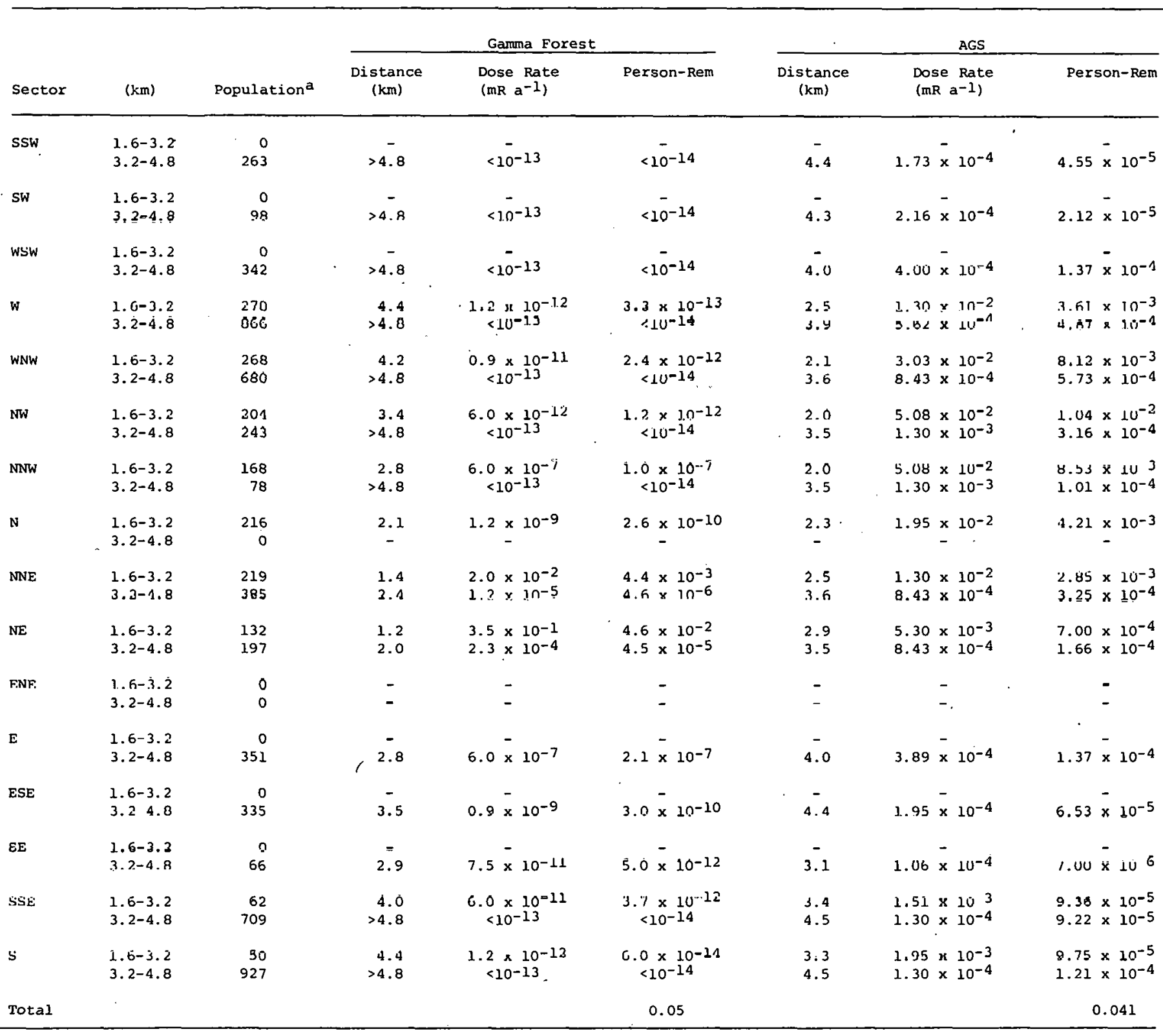

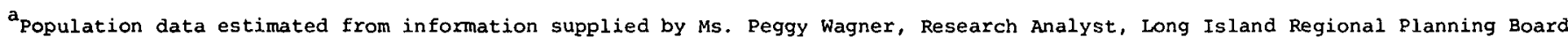
$[3,4]$. See Table 1 for estimated population distribution for 1979 . 


\subsection{Doses Due to Alternating Gradient Synchrotron}

The Alternating Gradient Synchrotron (AGS) is a $33 \mathrm{GeV}$ proton synchrotron located 1180 meters from the nearest site boundary. Although the machine is heavily shielded, some neutrons do penetrate the shield or escape from areas where experiments are in progress. Some of these neutrons reach off-site areas either directly or in most cases, by scattering from the air, which is called skyshine.

With the advent of the Isabelle project in 1978, the Safety and Environmental Protection Division has instituted an extensive program to evaluate different neutron detectors in the field and also to determine appropriate sampling locations. These studies should provide data on neutron dose distribution around AGS and Isabelle (when operational) and thus provide a basis for more accurate estimates of off-site doses. Preliminary results, derived by using a neutron monitor (Ludlum 55) at P2 (Figure 2), indicate an annual dose of 6.1 mRem resulting from neutrons other than those generated by the AGS facility. This dose rate has been observed to be similar to levels at other accelerator facilities (28). During 1978 and 1979, the study has been experimental in nature and as such it was felt that the neutron skyshine data for 1979 would not permit a proper evaluation of the doses off-site resulting from AGS operations. Accordingly, it was decided to estimate the dose rate at the site boundary by comparing the total proton flux for 1977 to that for 1978 and 1979 and use this ratio to derive the 1978 and 1979 dose rates from the 1977 values (Table 31 1977 E.M. Report). As such, Table 28 gives the derived dose rate (mRem $a^{-1}$ ) and the collective average dose equivalent (average doses) rates for each population segment and for each distance from the source.

Since the dose rate from this source decreases rapidly with distance, only population segments with radii of 1.6 to 3.2 and 3.2 to $4.8 \mathrm{kms}$ were considered. The off-site derived collective average dose equivalent (total dose) was $0.04 \mathrm{rem} \mathrm{a}^{-1}$ (person-rem $\mathrm{a}^{-1}$ ) and applicable contributions were found only in the NW and NNW sectors.

\subsection{Collective Average Dose Equivalent Rate (Total Population Dose)}

The collective average dose equivalent rate (total population dose) beyond the site boundary, within a radius of $80 \mathrm{~km}$, due to Laboratory operations during 1979 is the sum of the values due to the four components discussed above, as shown below: 
Airborne

Tritium $\quad 4.95$

Liquid Effluents

Consuming fish: Adults 0.35

Infants $\quad 0.05$

Well water 0.02

Gamma Forest Source 0.05

AGS Skyshine

Tota1

$\frac{0.04}{5.46}$

The collective average dose equivalent (total annual dose) due to external radiation from natural background, to the population within a $80 \mathrm{~km}$ radius of the Laboratory, amounts to about $278,405 \mathrm{rem} \mathrm{a}^{-1}$, to which about 85,704 rem $a^{-1}$ (person-rem $a^{-1}$ ), should be added for internal radioactivity from natural sourres. 


\begin{tabular}{|c|c|c|c|c|c|c|c|c|}
\hline \multicolumn{2}{|c|}{$\begin{array}{l}\text { Contaminant } \\
\text { Radionuclide }\end{array}$} & \multicolumn{2}{|c|}{ DOE 0524[17] } & $\begin{array}{l}\text { EPA-Drinking Water[18] } \\
\text { and NYS Drinking } \\
\text { Water Standard[25] (a) }\end{array}$ & \multicolumn{2}{|c|}{$\begin{array}{cr}\text { NYS Standard }[13] \\
\text { Air } & \text { Water }\end{array}$} & \multicolumn{2}{|c|}{$\begin{array}{ll}\text { Detection }_{\text {Limit }} & (\mathrm{b}) \\
\text { Ár } & \text { Water }\end{array}$} \\
\hline $\begin{array}{l}\text { Gross } \alpha \\
\mu \mathrm{C} 1 / \mathrm{ml}\end{array}$ & & $1 \times 10^{-13}$ & $6 \times 10^{-7}$ & $1.5 \times 10^{-8}$ & $1 \times 10^{-13}$ & $6 \times 10^{-7}$ & $3 \times 10^{-16}$ & $3 \times 10^{-10}$ \\
\hline $\begin{array}{l}\text { Gross } \beta \\
\mu \mathrm{C} 1 / \mathrm{ml}\end{array}$ & & $1 \times 10^{-10}$ & $1 \times 10^{-7}$ & $1 \times 10^{-7}$ & $1 \times 10^{-10}$ & $1 \times 10^{-7}$ & $1 \times 10^{-15}$ & $1 \times 10^{-9}$ \\
\hline $\begin{array}{l}{ }^{7} \mathrm{Be} \\
\mu \mathrm{Ci} / \mathrm{ml}\end{array}$ & $\begin{array}{l}S \\
I\end{array}$ & $\begin{array}{l}2 \times 10^{-7} \\
4 \times 10^{-8}\end{array}$ & $\begin{array}{l}2 \times 10^{-3} \\
2 \times 10^{-3}\end{array}$ & $\begin{array}{r}2 \times 10^{-3} \\
2 \times 10^{-3}\end{array}$ & $4 \times 10^{-8}$ & $2 \times 10^{-3}$ & $1 \times 10^{-12}$ & $5 \times 10^{-10}$ \\
\hline${ }^{3}{ }_{\mu \mathrm{Ci} / \mathrm{m} 1}$ & & $2 \times 10^{-7}$ & $3 \times 10^{-3}$ & $2 \times 10^{-5}$ & $2 \times 10^{-7}$ & $3 \times 10^{-3}$ & $2 \times 10^{-12(c}$ & c) $2 \times 10^{-11}$ \\
\hline $\begin{array}{l}{ }^{60} \mathrm{Co} \\
\mu \mathrm{Ci} / \mathrm{ml}\end{array}$ & $\begin{array}{l}S \\
I\end{array}$ & $\begin{array}{l}1 \times 10^{-8} \\
3 \times 10^{-10}\end{array}$ & $\begin{array}{lll}5 & \times 10^{-5} \\
3 \times & 10^{-5}\end{array}$ & $\begin{array}{l}5 \times 10^{-5} \\
3 \times 10^{-5}\end{array}$ & $3 \times 10^{-10}$ & $3 \times 10^{-5}$ & $1 \times 10^{-14}$ & $5 \times 10^{-10}$ \\
\hline${ }^{131} \mathrm{I}$ & $\begin{array}{l}S \\
\mathrm{I}\end{array}$ & $\begin{array}{l}1 \times 10^{-10} \\
1 \times 10^{-8}\end{array}$ & $\begin{array}{l}3 \times 10^{-7} \\
6 \times 10^{-5}\end{array}$ & $\begin{array}{l}3 \times 10^{-7} \\
6 \times 10^{-5}\end{array}$ & $1 \times 10^{-8}$ & $6 \times 10^{-5}$ & $1 \times 10^{-14}$ & $2 \times 10^{-10}$ \\
\hline $\begin{array}{l}{ }^{137} \mathrm{Cs} \\
\mu \mathrm{Cl} / \mathrm{ml}\end{array}$ & $\begin{array}{l}S \\
I\end{array}$ & $\begin{array}{l}2 \times 10^{-9} \\
5 \times 10^{-10}\end{array}$ & $\begin{array}{l}2 \times 10^{-5} \\
4 \times 10^{-5}\end{array}$ & $\begin{array}{l}2 \times 10^{-5} \\
4 \times 10^{-5}\end{array}$ & $2 \times 10^{-9}$ & $2 \times 10^{-5}$ & $1 \times 10^{-14}$ & $3 \times 10^{-10}$ \\
\hline${ }^{54} \mathrm{Mn}$ & $\begin{array}{l}S \\
I\end{array}$ & $\begin{array}{l}1 \times 10^{-8} \\
1 \times 10^{-9}\end{array}$ & $\begin{array}{l}1 \times 10^{-4} \\
1 \times 10^{-4}\end{array}$ & $\begin{array}{l}1 \times 10^{-4} \\
1 \times 10^{-4}\end{array}$ & $1 \times 10^{-8}$ & $1 \times 10^{-6}$ & $1 \times 10^{-14}$ & $3 \times 10^{-10}$ \\
\hline${ }^{90} \mathrm{Sr}$ & $\begin{array}{l}S \\
I\end{array}$ & $\begin{array}{l}3 \times 10^{-11} \\
2 \times 10^{-10}\end{array}$ & $\begin{array}{l}3 \times 10^{-7} \\
4 \times 10^{-5}\end{array}$ & $8 \times 10^{-9}$ & $3 \times 10^{-11}$ & $3 \times 10^{-7}$ & $1 \times 10^{-15}$ & $9 \times 10^{-14}$ \\
\hline
\end{tabular}

\section{Non-Radioactive}

Temp ${ }^{\circ} \mathrm{C}$

$\mathrm{pH}$

Dissolved Cxygen ppm Chlorides ppm

Nitrogen-Nitrate ppm Dissnlved Solids ppm Coliform

Ag ppm

Cd

$\mathrm{Cr}$

$\mathrm{Cu}$

$\mathrm{Fe}$

$\mathrm{Hg}$

$\mathrm{Pb}$

Zn
$\mathrm{T}_{\max }<30$

$$
\Delta \mathrm{T}=\stackrel{+}{-2.8}
$$

6.5-8.5

$>4.0$

250

10

500

Zero非/100ml

0.05

0.01

0.05

$-$

0.002

0.05

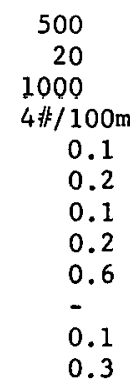

0.2

$\therefore 1$

.05

20.00

0.00

0.001

0.004

0.001

0.001

0.02

0.00007

0.005

0.002

a Aqquifer under Inng Tsland declared as "Sole Source" - Applicạble Sțandard 1s EPA National Interim Primary Drinking Water Regulations [18].

b See Appendix B.

c As tritiated vapor

S: Soluble

I : Insoluble 
APPENDIX A

QUALITY CONTROL

Radioactive Measurements

a. Alpha $(\alpha)$, Beta $(\beta)$ and Gamma $(\gamma)$ :

Certified radioactive standards from the National Bureau of Standards, U,S. Department of Commerce, are used to standardize radiation measurement instruments. These standards are certified to be at least within $5 \%$ of stated values. In some cases, certified standards were also obtained from Amersham/Searle. Daily checks of instrument performances are made using the standards as well as backgrounds. In addition, some samples are counted both in the $\mathrm{NaI}$ system and the $\mathrm{Ge}(\mathrm{Li})$ system. The $\mathrm{Ge}(\mathrm{Li})$ system were calibrated using a now multi-gamma NBS Standard obtaincd in 0etober 1977 . The results flum the $\mathrm{NaI}$ and $\mathrm{Ge}(\mathrm{Li})$ systems agree within $5 \%$. For tritium measurements a number of standards and blanks are included with each run of a liquid scintillator counter which has a programmed automatic sample changer.

The Analytical Laboratory of the Safety and Environmental Protection Division is a participant in the inter-laboratory comparisons of radioactivity in samples of different matrices of water, air filters, soil, vegetation and bone. These samples are distributed by the Department of Energy (DOE) through the Environmental Measurements Laboratory (EML), New York, formerly known as the Health and Safety Laboratory (HASL), on a quarterly basis. The radionucludes assayed were ${ }^{3} \mathrm{H},{ }^{90} \mathrm{Sr}$, plutonium isotopes (following wet chemistry) and a number of gamma emitting nuclides. Our results agree within $10 \%$ for water samples and within $15 \%$ for other sample matrices.

b. TLD Dosimeters

The Dosimetry Services Group of the Safety and Environmental Protection Division participated in the Fourth International Intercomparison of Environmental Dosimeters conducted at Houston, Texas during the late winter and early spring of 1979. There were a total of 122 participants in this test.

The estimated field exposure, as measured by the BNL. envirnnmenta1. monitoring TLD dosimeter, agreed within $0.8 \%$ of the value measured by a continuously operated recording pressurized ion chamber corrected for energy response. In the laboratory exposure test, the BNL dosimeter agreed within $3.5 \%$ for the "high" ( $~ 50 \mathrm{mR}$ exposure) and agreed within $6.4 \%$ for the "low" ( $15 \mathrm{mR}$ exposure).

Nonradioactive Measurements

Procedures for nonradioactive contaminants are those presented in Standard Methods for the Examination of Water and Wastewater (14th edition, 1975). All standards are prepared from standard reference grade and analytical grade reagents in accordance with the requirements of standard methods. Standards are run with each set of samples analyzed and at least one duplicate and blank is run with each set. 


\section{APPENDIX B}

inimum Detectable Limit (MDL)

Some of the values in gamma scans by the NaI detector are not indicated in the tables as these values were at or below MDL. The MDL values are a function of Matrix (efficiency), Count Time (background), etc. Typical tables for $\mathrm{NaI}$ and $\mathrm{Ge}(\mathrm{Li})$ systems are given below:

$$
\begin{array}{ll}
\text { Units : } & 10^{-6} \mu \mathrm{Ci} \\
\text { Detector: } & \text { Two } 4 " \mathrm{NaI} \text { crystals } \\
\text { Geometry: } & \text { Planchet and air particulates }
\end{array}
$$

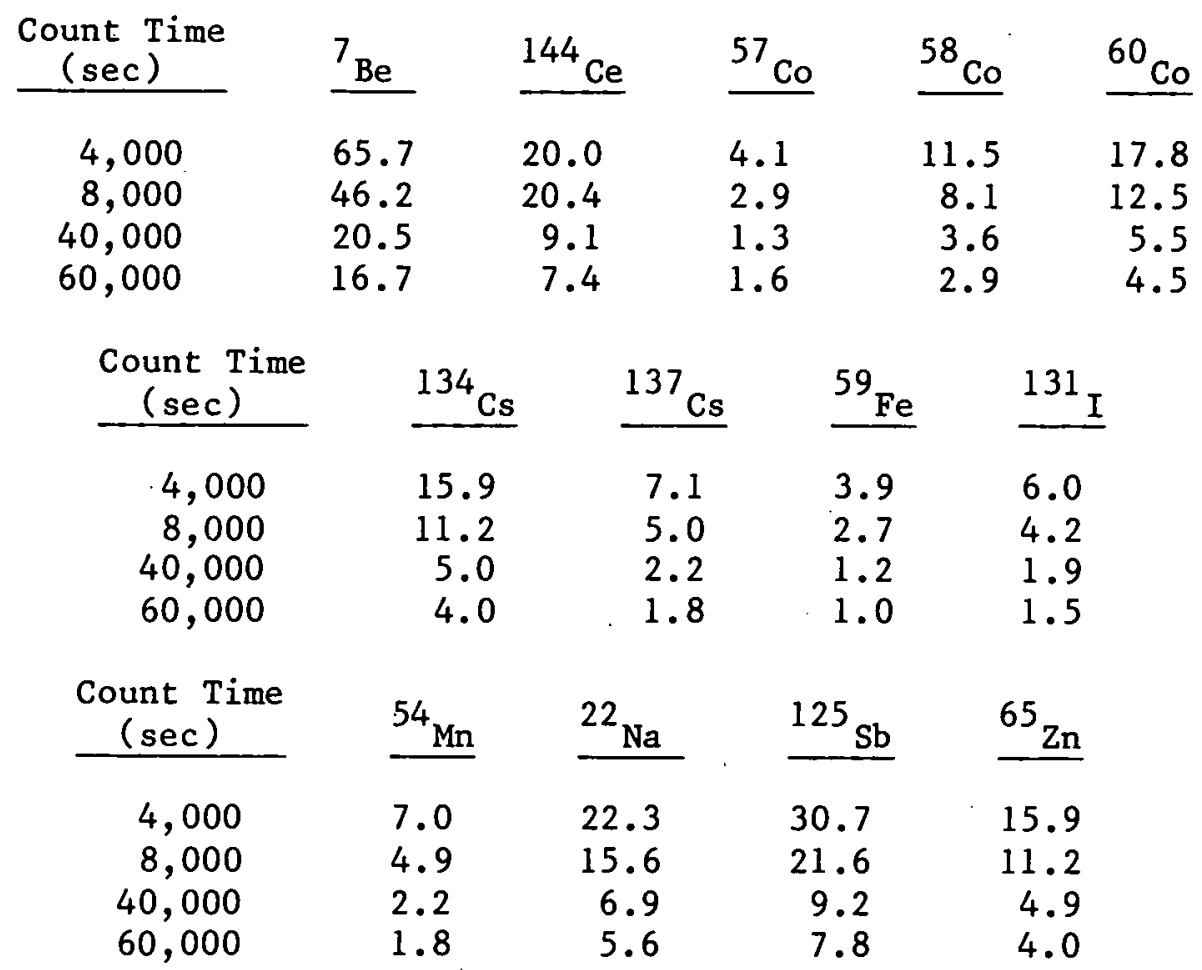

\begin{tabular}{|c|c|c|}
\hline$($ ser $)$ & ${ }^{7} \mathrm{Be}$ & ${ }^{144} \mathrm{Ce}$ \\
\hline $\begin{array}{r}4,000 \\
50,000\end{array}$ & $\begin{array}{r}18.5 \\
7.5\end{array}$ & $\begin{array}{l}8.2 \\
4.1\end{array}$ \\
\hline
\end{tabular}

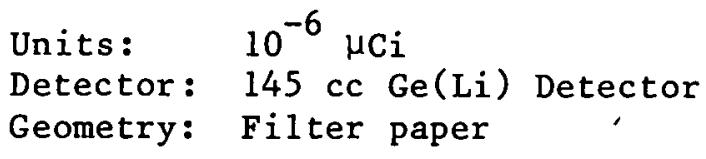

Count Time 


\begin{tabular}{|c|c|c|c|c|}
\hline $\begin{array}{c}\text { Count Time } \\
(\mathrm{sec})\end{array}$ & ${ }^{134} \mathrm{Cs}$ & ${ }^{137} \mathrm{Cs}$ & ${ }^{59} \mathrm{Fe}$ & $131_{I}$ \\
\hline 4,000 & 7.5 & 3.2 & 1.2 & 2.7 \\
\hline 50,000 & 2.1 & 1.0 & 0.6 & 0.8 \\
\hline $\begin{array}{l}\text { Count Time } \\
(\mathrm{sec})\end{array}$ & ${ }^{54} \mathrm{Mn}$ & ${ }^{22} \mathrm{Na}$ & ${ }^{125} \mathrm{Sb}$ & ${ }^{65} \mathrm{Zn}$ \\
\hline 4,000 & $3: 1$ & 8.6 & 12.8 & 6.8 \\
\hline 50,000 & 1.0 & 2.1 & 3.1 & 2.3 \\
\hline
\end{tabular}




\section{ACKNOWLEDGMENTS}

The author would like to take this opportunity to express his sincere thanks to the following people who were responsible for data reduction and tabulation: A. Kuehner, N. Fallon, and S. Zambelli. They were prompt, efficient, and did an excellent job of a difficult task.

To the following people who, in spite of their busy schedule, took time to review the report and offered their valuable comments, a sincere vote of thanks; the quality of the report has definitely been enhanced through their suggestions: J. Baum, R. Casey, L. Emma, A Hu11, E. Hartmann, L. Kalbach, A. Kuehner, E. Lessard, C. Meinhold, R. Miltenberger, A. Moorthy, L. Olmer, L. Phillips, N. Rohrig, J. Steimers, M. Varma, 0. White, J. Hennessy (P1ant Engineering), and H. Manalastas (Philippine Atomic Energy Commission).

Finally, there are numerous people in Safety and Environmental Protection and throughout the Laboratory who have, in some form or other, assisted in providing the necessary information. Their cooperation is gratefully acknowledged.

This report was typed by members of the Word Processing group. Their painstaking effort is commended and recognized. Special thanks goes to Jodi Earle for her patience in helping us with all the minute details that go into completing the report. 


\section{REFERENCES}

1. U.S. Energy Research and Development Administration, Effluent and Environmental Monitoring and Reporting, ERDA Manual, Chapter 0513 and Appendix 0513 (1974).

2. U.S. Energy Research and Development Administration, A Guide for Environmental Radiological Surveillance at ERDA Installations, ERDA-77-24 (March 1977).

3. Nassau-Suffolk Regional Planning Board; U.S. Census - 1970 (October 1971).

4. LILCO, Population Survey - 1979 (January 1980).

5. M.A. Warren, W. de Laguna, and N.J. Lusczynski, Hydrology of Brookhaven National Laboratory and Vicinity, Geo. Survey Bull. 1156-C (1968).

6. P.H. Cohen et al., Atlas of Long Island Water Resources, New York State Resources Bull. No. 62 (1969).

7. D.B. Clearlock and A.F. Reisenauer, Sitewide Ground Water Flow Studies for Brookhaven National Laboratory, BNL Informal Report, December (1971).

8. U.S. Department of Energy, Proton-Proton Storage Accelerator Facility (ISABELLE), DOE/EIS-0003 (August 1978).

9. F.P. Cowan and C.B. Meinhold, Radiation dosimetry for ${ }^{60}$ Co and ${ }^{137}$ Cs gamma ray field irradiation facilities. Radiation Botany, Vol. 2, pp. 241-249, 1962.

10. D.M. Denham, et al., A CaF 2 :Dy 'lhermolumlnescent dosimeler for environmen tal monitoring. BNWL-SA-4191 (1972).

11. Compilation of Air. Pollutant Emission Factors, Envirulmental Frotection Agency, AP-42 (1975).

12. National Primary and Secondary Air Water Quality Standards, 40 CFR 50, 36 FR 22384, 11/25/71 and 38 FR 25678, 9/14/73.

13. Classification and Standards Governing the Quality and Purity of Waters of New York State, Parts 700-703, New York State Department of Environmental Conservation (1967).

14. Summary of Selected Pest Control Practices Proposed for 1976, ERDA Report to the Federal Working Group on Pest Management, Internal ERDA Report (1976).

15. Safety Manual, Brookhaven National Laboratory (1978).

16. M.M. Sma11, National Sewage Recycling Systems. BNL 50630 (1977). 
17. Standards for Radiation Protection, ERDA Manua1, Chapter 0524, Appendix A, Table II, Guide for Uncontrolled Areas (1968).

18. Environmental Protection Agency National Interim Primary Drinking Water Regulations, 40 CFR 141; 40 FR 59565, December 25, 1975; amended by 41 FR 28402, July 9, 1976.

19. Recommended Classifications and Assignment of Standards of Quality and Purity for Designated Waters of New York State, Part 921, New York State Department of Environmental Conservation (1967).

20. M. Schnitzer and S.U. Kahn, Humic Substances in the Environment. 327 pp. Marcel Dekker, New York (1972).

21. Criteria Governing Thermal Discharge, Part 704, Regulation of New York State Department of Environmental Conservation (1974).

22. P.F. Gustafson et al., Behavior of Fallout ${ }^{137} \mathrm{Cs}$ in Aquatic and Terrestrial Environments, ANL-7615 (1969).

23. W.H. Chapman, H.L. Fisher, and M.W. Pratt, Concentration Factors of Chemical Elements in Edible Aquatic Organisms, UCRL-50564, December 1968.

24. U.S. Nuclear Regúlatory Commission Regulatory Guide 1.109, Calculation of Annual Doses to Man from Routine Releases of Reactor Effluents for the Purpose of Evaluating Compliance with 10 CFR Part 50, Appendix I, Revision 1 - October 1977 .

25. Safe Drinking Water Act, New York State-Section 1424 (e): Aquifier Underlying Nassau and Suffok Counties (NYS) designated as a sole source.

26. G.M. Woodwell et al., Experimental Eutrophication of Terrestrial and Aquatic Ecosystems, Annual Report of the Upland and Recharge Project, BNL 50420(1974).

27. Holzworth, Mixing Heights Wind Speeds and Potential for Urban Air Pollution, Preliminary EPA Document, May 10, 1971.

28. National Council on Radiation Protection. Natural Background Radiation in the United States. Report No. 45., November 15, 197.5. 


\section{Distribution}

Internal

R. B. Aronson, Medical

V. P. Bond, Associate Director

R. H. Browne, AGS

L. C. Emma, DO

F. K. German, Biology

J. J. Hennessey, Plant Engr.

W. Y. Kato, DEE

B. Manowitz, DEE

C. B. Meinhold, S\&EP
R. A. Michae1, DEE

P. V. Mohn, AGS

S. C. Morris, DEE

G. C. Kinne, Reactor

M. J. Rose, Plant Engr.

V. L. Sailor, DEE

E. E. Shelton, Plant Engr.

L. G. Stang, Medical

R. W. Stoenner, Chemiotry

C. Thein, Public Relations

\section{External}

$\ddot{B H}=D O E$ Operationo

CH-DOE Operations

A. Andrioli, Suffolk County Dept of Health

M. Awschalom, Fermilab

J. W. Bair, BNWL

N. Barr, DOE-DBER

S. Becker, Suffolk County Dept. of Health

G. Brezner, NYS Dept. of Environmental Conservation

W. W. Burr, DOE-DBER

T. Cashman, NYS Dept. of Environmental Conservation

M. Cordaro, LILCO

J. P. Corley, BNWL

D. N. Edgington, ANL

M. Eisenbud, NYU Medical Center, Sterling Forest

J. Feldulal, New York Reglonal office, USEYYA

H. Fischer, Suffolk County Council on Environmental Quality

J. J. Fixx, BNWL

J. Foehrenbach, New York State Conservation Dept.

R. Gardner, SUNY, Stony Brook

C. Gratham, G\&S Associate

P. Gudiksen, LLL

E. Gupton, ORNL

J. Harley, EML

E. P. Hardy, EML

J. Hunter, Rutgers Üniversity

L. Johnson, LASL

I. Kantrowitz, U. S. Geological Survey

L. Koppelman, Nassau-Suffolk Regional Planning Board

C. S. Larson, Suffolk County Council on Environmental Quality 
C. L. Lindeken, LLL

J. Logsdon, Radiation Safety Program, EPA

P. Lorio, Columbia University

H. McCammon, DOE-DBER

J. McLaughlin, EML

A. Nelson, LILCO

E. O'Connell, SUNY, Stony Brook

C. M. Patterson, SRL

H. W. Patterson, LLL

G. Proios, Town of Brookhaven

D. Puleston, EDF

W. C. Reinig, SRL

W. Roberts, Suffolk County Dept. of Health

J. D. Sage, BAPL

L. Salzman, Friends of the Earth

T. H. Schoenberg, KAPL

J. Sedlet, ANL

A. Sielman, League of Women Voters, Suffolk County

C. W. Sill, Idaho-HASL

D. H. Slade, DOE-DBER

R. Smolker, Brookhaven Town Board, Waterways \& Natural Resources

J. D. Soldat, BNWL

L. Solon, New York City Dept. of Radiological Control

C. Stern, Long Island Environmental Council, Inc.

J. P. Stohr, NRC Region I

J. Swineboard, DOE-DBER

A. Taormina, New York Dept. of Environmenta1. Conservation

R. Thomas, LBL

C. M. Unruh, BNWL

G. L. Voelz, LLL

R. Wood, DOE-DBER

A. Yerman, NYS Dept. of Environmental Conservation

M. Zaki, Suffolk County Health Department 